\title{
Double emulsions as fat replacers \\ Linking emulsion design to stability and sensory perception
}

Anika Oppermann 


\section{Thesis committee}

\section{Promotor}

Prof. Dr C. de Graaf

Professor of Sensory Science and Eating Behaviour

Wageningen University \& Research

\section{Co-promotors}

Dr M.A. Stieger

Associate professor, Division of Human Nutrition

Wageningen University \& Research

Dr E. Scholten

Associate professor, Physics and Physical Chemistry of Foods

Wageningen University \& Research

\section{Other members}

Prof. Dr C.G.P.H. Schroën, Wageningen University \& Research

Prof. Dr P. Wilde, Institute of Food Research, Norwich, United Kingdom

Dr F. van de Velde, NIZO food research, Ede, The Netherlands

Dr R.A. de Wijk, Wageningen Food \& Biobased Research

This research was conducted under the auspices of the Graduate School VLAG (Advanced studies in Food Technology, Agrobiotechnology, Nutrition and Health Sciences). 


\section{Double emulsions as fat replacers \\ Linking emulsion design to stability and sensory perception}

\section{Anika Oppermann}

Thesis

submitted in fulfilment of the requirements for the degree of doctor at Wageningen University

by the authority of the Rector Magnificus

Prof. Dr A.P.J. Mol,

in the presence of the

Thesis Committee appointed by the Academic Board

to be defended in public

on Friday, $3^{\text {rd }}$ of March 2017

at 1:30 p.m. in the Aula. 


\section{Anika Oppermann}

Double emulsions as fat replacers - Linking emulsion design to stability and sensory perception

188 pages.

PhD thesis, Wageningen University, Wageningen, NL (2017)

With references, with summary in English

ISBN: 978-94-6343-072-2

DOI: http://dx.doi.org/10.18174/403479 
Für meine Familie 



\section{Table of contents}

\section{Page}

Chapter 1 General introduction

$\begin{array}{lll}\text { Chapter } 2 & \text { Effect of gelation of inner dispersed phase on } & 17\end{array}$ stability of double $\left(\mathrm{w}_{1} / \mathrm{o} / \mathrm{w}_{2}\right)$ emulsions

Chapter 3 Effect of outer water phase composition on oil droplet size and yield of double $\left(\mathrm{w}_{1} / \mathrm{o} / \mathrm{w}_{2}\right)$ emulsions

Chapter 4 Influence of double $\left(\mathrm{w}_{1} / \mathrm{o} / \mathrm{w}_{2}\right)$ emulsion composition on lubrication properties

Chapter 5 Descriptive sensory profiling of double emulsions with gelled and non-gelled inner water phase

Chapter 6 Comparison of Rate-All-That-Apply (RATA) and

Descriptive sensory Analysis (DA) of model double emulsions with subtle perceptual differences

Chapter 7 General discussion

Summary

Acknowledgements

About the author 




\subsection{General introduction}

Obesity is a major risk factor for nutrition-related chronic diseases such as diabetes, cardiovascular diseases, and several cancers [1]. A major cause of overweight and obesity has been shown to be an increased intake of energy-dense foods in combination with insufficient physical activity [1]. Therefore, there is a need for the development of reformulated, fat-reduced food products to contribute to lowering consumers' energy intake. Reducing the oil content in food is a logical step as oil contributes with $9 \mathrm{kcal} / \mathrm{g}$ to the energy density of foods, which is more than twice the energy density compared to proteins or carbohydrates $(4 \mathrm{kcal} / \mathrm{g})$. Of specific interest in this thesis are food emulsions like dairy products (milk, yoghurt, cream), sauces, soups, spreads, desserts, and dressings (e.g., mayonnaise), which contain small oil droplets dispersed in water.

\subsection{Functionality of fats in foods}

Reduction of fat content within food products often deteriorates its desirable quality attributes (e.g. appearance, texture, stability, flavour profile, and satiety response) [2]. This decrease in quality aspects can be compensated by the use of fat replacers. In order to develop effective fat replacers, an understanding of the different functions of fats and oils in food emulsions is required. Fats and oils have a major influence on 1) physicochemical product properties, 2) sensory perception, and 3) physiological responses to ingested foods [3-5].

In oil-in-water $\left(\mathrm{o} / \mathrm{w}_{2}\right)$ emulsions, the oil is dispersed in the form of small droplets in a continuous aqueous phase. The emulsions' physicochemical properties comprise appearance (colour and opacity), rheological and tribological properties, as well as emulsion stability. These physicochemical properties are determined by fat content, droplets physical state (solid or liquid), droplet size and distribution, properties of the $\left(\mathrm{o} / \mathrm{w}_{2}\right)$ interface and other components present in the emulsions such as salts and thickeners $[2,6-10]$.

The sensory perception of food emulsions is affected by these physicochemical emulsion properties. Sensory perception includes appearance, flavour (i.e. taste and aroma), texture and mouthfeel. In particular, fat-related sensory perception of food emulsions, such as fattiness, creaminess, smoothness and thickness, is influenced by the type and amount of dispersed fat or oil [6, 9, 11, 12]. For example, emulsion viscosity, influenced by the dispersed fat content, affects perceived thickness, fattiness and creaminess $[6,9,12]$. Several studies have shown that at different stages of oral 
processing, both rheological as well as lubrication properties affect the fat-related sensory perception [13, 14]. Fat is considered a good lubricant since it decreases the friction between the tongue and palate, thereby contributing to a fatty and creamy perception through lubrication [7, 15-18]. It has been suggested that fat-related sensory perception is mostly influenced by the contact of oral surfaces with the fat droplets [19].

From a physiological perspective, fats do not only provide energy required to maintain body functions, but are also essential structural elements of cell-surrounding membranes, and can transport fat-soluble vitamins A, D, E and K [20-22].

Since emulsified fats fulfil different roles in foods, fat reduction is a complex challenge. Fat reduction is successful only when the physicochemical and sensory properties of a reduced-fat product resemble or excel those of the full-fat counterpart.

\subsection{Common fat reduction strategies in foods}

A variety of approaches exists to develop fat-reduced products that have comparable texture, mouthfeel and/or flavour profiles as their full-fat counterparts. These approaches can be based on changing (1) the composition, (2) the process or (3) the structure of the foods.

With regard to changes in composition, common fat replacers can partially mimic certain desired characteristics, but may lack other functions provided by fat. For example, polysaccharides can increase the viscosity of emulsions, but might not provide a similar contribution to flavour perception as fat [23]. Other types of fat replacers are particulates (starch granules, hydrogel beads, protein microspheres/ micro-particulated proteins, or air bubbles), and fat-mimetics (e.g. sucrose fatty acid polyesters) [24]. Micro-particulated proteins, for example, are small, spherical particles that mimic the mouthfeel and texture of fat, but might mask flavour [25-27]. Sucrose fatty acid polyesters, also known as Olestra, are non-caloric and not absorbed during digestion. Although they may provide similar mouthfeel as fat, they can negatively affect the digestion in the form of abdominal cramps and soft stools, and reduce absorption of fat-soluble vitamins A, D, E and K [28]. Overall, common fat replacers can, to some extent, replace some functions of fat, but usually not all of them [28].

Other fat reduction strategies do not change the product composition but focus on changes in the processing or structuring of the food. The use of novel processing techniques, such as microfluidization, is one example of changing the process as a strategy to reduce fat content $[29,30]$. This technique decreases the droplet size and 
consequently increases the surface area of fat that is in contact with the oral surfaces. Food structuring approaches, in contrast, make use of changes in the microstructure of the food to enhance fat-related sensory perception while the actual fat content is reduced. For example, it has been shown that coalescence of fat droplets during oral processing, which depends on the type of fat and emulsifier used, can be used to increase perception of fat-related attributes, such as creaminess $[6,7,13,31,32]$.

Even though several fat reduction strategies exist, fat-reduced products rarely match the physicochemical and sensory properties of full-fat counterparts. Particularly the mouthfeel and flavour perception are often not matched [11]. This may be a consequence of a decrease in lubrication in the oral cavity, since less oral surfaces are in contact with fat. Since consumer acceptance of food products highly depends on the product and its sensory properties, there is a need to investigate approaches in which the contact between oil and oral surfaces in the mouth is maintained.

\subsection{Fat reduction in foods using double $\left(w_{1} / 0 / w_{2}\right)$ emulsions}

A structuring approach to lower fat content in emulsions and emulsion-based foods while maintaining the contact area between fat and oral surfaces in the mouth, is the use of double $\left(w_{1} / o / w_{2}\right)$ emulsions. In comparison to single $\left(o / w_{2}\right)$ emulsions, in which oil is dispersed in form of droplets in a continuous water phase $w_{2^{\prime}}$ double $\left(w_{1} / o / w_{2}\right)$ emulsions contain an extra phase, namely small $w_{1}$ droplets inside the larger oil droplets. These small water droplets replace part of the oil while maintaining the total oil droplet surface area (Figure 1.1).

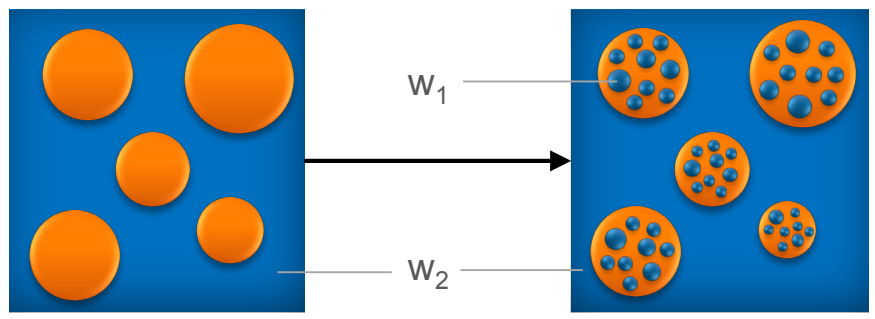

Figure 1.1 Replacement of oil by dispersed water droplets $w_{1}$ in double $\left(w_{1} / 0 / w_{2}\right)$ emulsions

\subsubsection{Stability of double $\left(w_{1} / o / w_{2}\right)$ emulsions}

To prepare double emulsions, many of the same tools used for preparation of conventional emulsions such as high shear mixers, high pressure homogenizers, sonicators and membrane homogenizers can be used [33, 34]. Generally, double emulsions are prepared in a two-stage process [35, 36]. First, a primary water-in-oil $\left(w_{1} / 0\right)$ emulsion is prepared under high shear to obtain small water droplets (typically 
in the submicron range), before dispersing and emulsifying the primary $\left(w_{1} / 0\right)$ emulsion in an outer water phase under low shear conditions to avoid extensive oil droplet breakup. To retain a substantial amount of water droplets inside the oil droplets, oil droplets in double emulsions usually have a larger size than in single emulsions (an average size of $50 \mu \mathrm{m}$ is common) [37].

To stabilize the two interfaces $\left(w_{1} / o\right.$ and $\left.o / w_{2}\right)$, two types of emulsifiers are needed. To stabilize the $\left(\mathrm{w}_{1} / \mathrm{o}\right)$ interface, a lipophilic emulsifier, such as polyglycerol polyricinoleate (PGPR), is used. To stabilize the $\left(0 / w_{2}\right)$ interface, the same hydrophilic emulsifiers as in single $\left(\mathrm{o} / \mathrm{w}_{2}\right)$ emulsions can be used. For example, proteins such as whey protein isolate (WPI) or Na-caseinate, phospholipids and low-molecular weight emulsifiers can be used to stabilize the oil droplets [38].

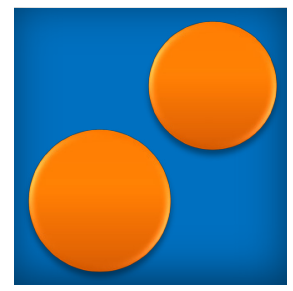

Coalescence

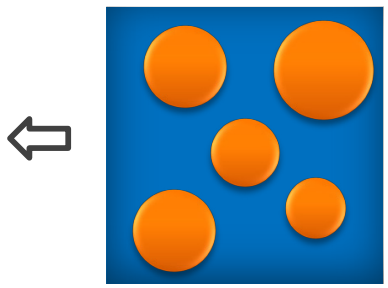

L
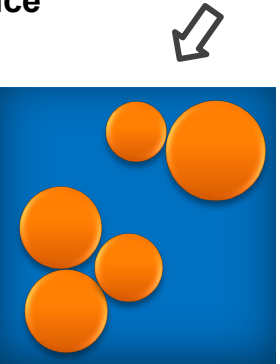

Flocculation

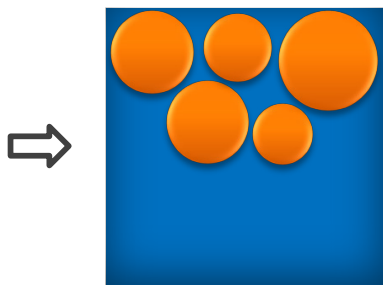

Creaming 
For large emulsion droplets, as usually present in double emulsions, creaming rate is therefore high. Emulsions can also become unstable when droplets are able to approach each other. Droplets in double emulsions can then flocculate or coalesce depending on the type of emulsifiers used and the interactions between the droplets [39]. Ostwald ripening can occur when the droplet size distribution is very broad, leading to coarsening of the emulsion.

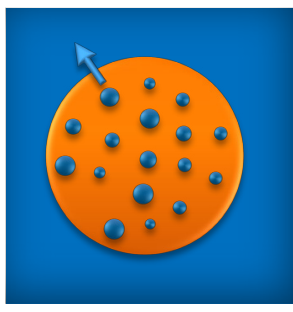

Diffusion

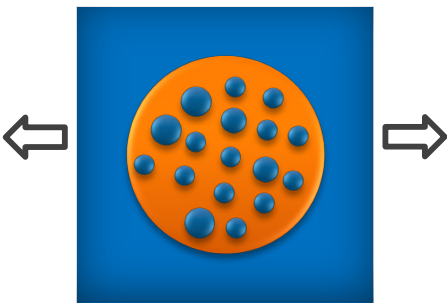

Figure 1.3 Loss of inner aqueous phase by diffusion of water from $w_{1}$ to $w_{2}$ or coalescence between $\mathrm{w}_{1}-\mathrm{w}_{2}$

As depicted in Figure 1.3, destabilization mechanisms in double emulsions are more complex. This is related to the presence of the additional dispersed aqueous phase $\left(w_{1}\right)$ and the two types of interfaces $\left(0 / w_{2}\right.$ and $\left.w_{1} / o\right)$. In double $\left(w_{1} / 0 / w_{2}\right)$ emulsions, the inner aqueous $w_{1}$ droplets are susceptible to different destabilization processes. They may destabilize due to coalescence of inner water droplets with the outer aqueous phase or due to diffusion of water from the inner to the outer aqueous phase (or vice versa) [40]. Two mechanisms are suggested to be dominant for the diffusion of water through the oil phase: a) reverse micellar transport, and b) diffusion of water molecules across areas where the oil layer between the two aqueous phases is thin [41-44]. Diffusion of water between the two aqueous phases through the oil layer is induced by a pressure gradient between the outer water phase and the small water droplets. Small water droplets experience larger pressures, commonly known as the Laplace pressure $P(P=2 \gamma / r$, with $\gamma$ denoting the interfacial tension and $r$ denoting the droplet radius). High pressure promotes migration of water from the inner $\mathrm{w}_{1}$ droplets to the outer $\mathrm{w}_{2}$ continuous phase. The Laplace pressure within inner $\mathrm{w}_{1}$ droplets can be counterbalanced by the addition of solutes influencing the osmotic pressure of the inner $w_{1}$ droplets $[42,44-47]$. Due to an increased osmotic pressure in the inner water droplets, a negative osmotic pressure gradient is created, promoting the reverse migration of water from the outer $w_{2}$ to the inner $w_{1}$ phase. By balancing the Laplace and osmotic pressure of the inner aqueous phase, loss of water by diffusion from $w_{1}$ to $\mathrm{w}_{2}$ can be minimized $[42,48-50]$. 
Double $\left(w_{1} / 0 / w_{2}\right)$ emulsions are often characterized by the amount of water $w_{1}$ (or a certain compound) inside the oil droplets, which can be interpreted as the fat reduction level. The amount of water that remains in the oil droplets depends on the composition and production process, and is defined as yield or encapsulation efficiency and expressed as a percentage. Throughout this thesis, yield is used as the parameter describing the percentage (or fraction) of inner aqueous phase entrapped in the oil phase at a certain time point, compared to the percentage (or fraction) of inner water droplets of the initial primary $\left(w_{1} / 0\right)$ emulsion.

\subsubsection{Challenges of using double $\left(w_{1} / 0 / w_{2}\right)$ emulsions as fat replacers}

So far, double emulsions have rarely been applied in food products [51,52]. The main reason is the susceptibility of double emulsions to release inner water droplets during preparation, storage and under environmental stresses commonly occurring in food production (mechanical forces, thermal processing, and chilling) [40]. However, extensive research has already been performed on the preparation and stabilization of double emulsions. The influence of type of hydrophilic emulsifiers and addition of biopolymers to the inner or outer aqueous phase on physicochemical properties of double emulsions has been investigated [45, 50, 53-69]. It has been shown that stability can be increased by thickening or gelling the inner water droplets $[45,63$, $64,70]$. However, not much is known about the effect of gelation and the mechanical properties of the gelled droplets on yield and stability of double emulsions. Only a few studies have been performed on how emulsions can be designed to remain stable not only during preparation or storage, but also during processes such as shear or heat treatment [63].

To reduce creaming of the large dispersed $\left(w_{1} / 0\right)$ droplets in double emulsions, the viscosity of the continuous phase can be increased to limit the creaming. However, little is known about the influence of the addition of thickeners to the outer water phase on the interfacial properties of the oil-water $\left(\mathrm{o} / \mathrm{w}_{2}\right)$ interface and consequently the oil droplet size and level of fat reduction that can be achieved. It should be noted that dispersed $\left(w_{1} / 0\right)$ droplets cannot be very small as they would lose their inner water droplets through $w_{1}-w_{2}$ coalescence as collision probability with the outer water phase increases with decreasing oil droplet size. 


\subsection{Sensory perception of single $\left(0 / w_{2}\right)$ and double $\left(w_{1} / 0 / w_{2}\right)$ emulsions}

For a fat reduction strategy to be successful, mouthfeel and texture perception of the reduced-fat emulsions or emulsion-based foods should be similar to those of their full-fat counterparts. As described previously, fat contributes to thickness, fattiness and creaminess perception, and is linked to the contact of oral surfaces with fat droplets. For single $\left(\mathrm{o} / \mathrm{w}_{2}\right)$ emulsions, it is known that oil volume fraction, solid fat content, and possibly oil droplet size influence perception of fat-related sensory attributes $[6,9,11,12]$. In contrast to the extensive literature on sensory perception of single emulsions and emulsion-filled gels, the texture and mouthfeel perception of double emulsions has not been described yet. Since double $\left(w_{1} / 0 / w_{2}\right)$ emulsions have a similar total oil droplet surface area as full-fat $\left(\mathrm{o} / \mathrm{w}_{2}\right)$ emulsions in the case of similar oil droplet sizes, it is hypothesized that sensory perception of double emulsions is similar to that of their full-fat counterparts [51]. For the development of double emulsions as potential fat replacers, it is essential to gain more insights in the fatrelated sensory perception of double $\left(\mathrm{w}_{1} / \mathrm{o} / \mathrm{w}_{2}\right)$ emulsions.

\subsection{Tribological properties of single $\left(o / w_{2}\right)$ and double $\left(w_{1} / o / w_{2}\right)$ emulsions}

As introduced in section 1.2, both the rheological properties and the lubrication properties of emulsions contribute to the sensory perception of emulsion-based foods. Lubrication is measured with thin-film rheology, also known as tribology [71]. In tribological measurements, the friction force between two parallel surfaces in relative motion in the presence of a product, e.g. an emulsion, is studied. Lubrication increases when the friction force decreases. The importance of frictional forces and lubrication on texture perception was recognized by Kokini and Cussler [72] and has become popular fairly recently $[73,74]$. Tribology provides information about food products when surface properties are dominant [75]. To study lubrication properties of foods, traditional hard metallic surfaces are replaced by soft elastomers to mimic the low pressure conditions present in oral surfaces [76].

The friction coefficient $\mu$ expresses the ratio of the frictional force to the force (load) applied perpendicular to the two surfaces of the tribo-pair. A typical example of the friction coefficient as a function of the entrainment speed of the surfaces times viscosity divided by the load is depicted as a Stribeck curve in Figure 1.4. Usually, three regimes are identified in a Stribeck curve: the boundary, the mixed and the hydrodynamic lubrication regime. At very low entrainment speeds, the two surfaces 
are in contact and the lubricant is typically not entrained. This results in high friction between the two surfaces. This regime is called boundary regime. With increasing entrainment speeds, sufficient amount of lubricant enters the gap between the two surfaces. A coherent lubricating film is obtained, and this regime is the mixed lubrication regime. In this regime, the film thickness increases with increasing entrainment speed, separating the two surfaces more. This decreases the friction between the surfaces and consequently the friction coefficient. With a further increase in entrainment speed, the film thickness increases further as the liquid is able to push the two surfaces further apart. At this point, the load is reduced and not the surface properties but the bulk properties (viscosity) dominate the friction. In this hydrodynamic regime, the friction therefore increases again.

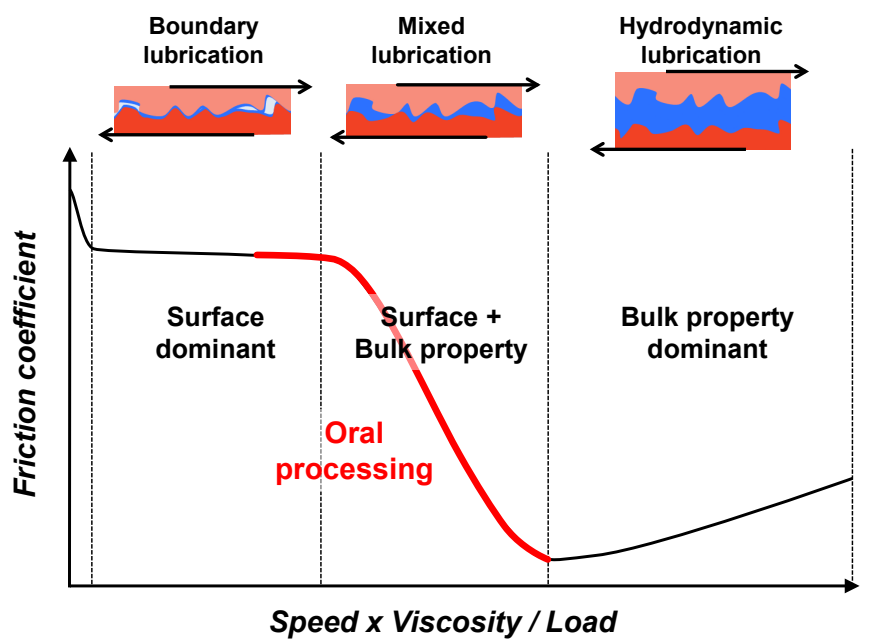

Figure 1.4 Typical Stribeck curve showing the three lubrication regimes. Reproduced and modified with permission from Liu [19]

For single $\left(\mathrm{o} / \mathrm{w}_{2}\right)$ emulsions, it is known that particularly the fat content influences friction [7, 15, 77-79]. Generally, friction decreases with increasing fat content. It is believed that oil is able to form an oil film or oil film patches providing lubrication $[26,80]$. The coalescence of oil droplets, either controlled by the type and concentration of emulsifier or the solid fat content of fat droplets, reduces friction by forming such an oil film $[6,7,13,31,32]$. Several authors linked friction measurements of model food systems (such as mayonnaise, chocolate and desserts) to texture perception [16, 78, 81]. For example, low friction was linked to smoothness [82], creaminess, fattiness and spreadability [13], while high friction was linked to roughness in different foods [16]. In contrast to several studies on the frictional behaviour of single emulsions, the frictional behaviour of double emulsions has not been studied yet. Currently it is 
not known to which extent the presence of small water droplets in double emulsions influences the lubrication properties, and how lubrication properties of double emulsions can be linked to sensory perception. Insights in the lubrication behaviour therefore provide understanding for the application of double emulsions as fat replacers in emulsions and emulsion-based foods.

\subsection{Comparison of sensory methodologies using trained and untrained subjects}

To obtain detailed, consistent and reliable information on the sensory perception of food products, usually Descriptive sensory Analysis (DA) with trained panels is used $[83,84]$. The disadvantage of trained sensory panels is that they are time- and resource intensive to set up and maintain. Therefore, recently novel sensory techniques focussing on sensory profiling using untrained participants have been developed $[85,86]$. The potential of sensory profiling techniques involving untrained participants lies in the reduced time investment, lower costs, and less training requirements. One promising sensory methodology using untrained participants is Rate-All-ThatApply (RATA), an intensity-based variant of the Check-All-That-Apply (CATA) method. In CATA, participants select from a list of attributes those sensory attributes that are applicable to describe the sensory properties of the food product. In RATA, this is followed by a rating of the perceived intensity of the selected attributes [87]. So far, studies comparing DA with CATA and RATA have been performed on foods with obvious and large differences in sensory characteristics [87-89]. DA with a trained panel is often used to describe the sensory properties of foods with small and subtle perceptual differences [83]. It is not known whether CATA and RATA with untrained subjects can be employed to reliably describe the sensory properties of model foods with small and subtle perceptual differences. It is therefore important to investigate whether RATA can be used with untrained participants when sample differences are very subtle and participants are not familiar with the stimulus. Double emulsions are very suitable as a model food in such studies, since their sensory perception is expected to differ only slightly from that of full-fat single emulsions.

\subsection{Objectives and thesis outline}

Current knowledge is limited on how to enhance the fat reduction level in double $\left(w_{1} / o / w_{2}\right)$ emulsions while at the same time maintaining their structural integrity during processing and other conditions. To gain insights in these aspects, understanding the influence of certain ingredients, such as emulsifiers and thickeners, as well as 
the inner $w_{1}$ droplet properties, on double emulsion composition and lubrication is essential. Furthermore, further understanding of the relationship between double emulsion composition and sensory perception is required for the development of double emulsions as fat replacers. To use double emulsions as fat replacers in foods, double emulsions should fulfil the following requirements:

1. high levels of fat reduction

2. stability against conditions encountered during processing and storage

3. mouthfeel and sensory perception similar to that of full-fat equivalents

The aim of this thesis was to better understand the influence of composition and structure of double $\left(w_{1} / 0 / w_{2}\right)$ emulsions on physicochemical properties, stability against processing conditions and sensory perception.

This thesis is structured as follows. The relationship between the chapters and the different parameters and properties studied is illustrated in Figure 1.5.

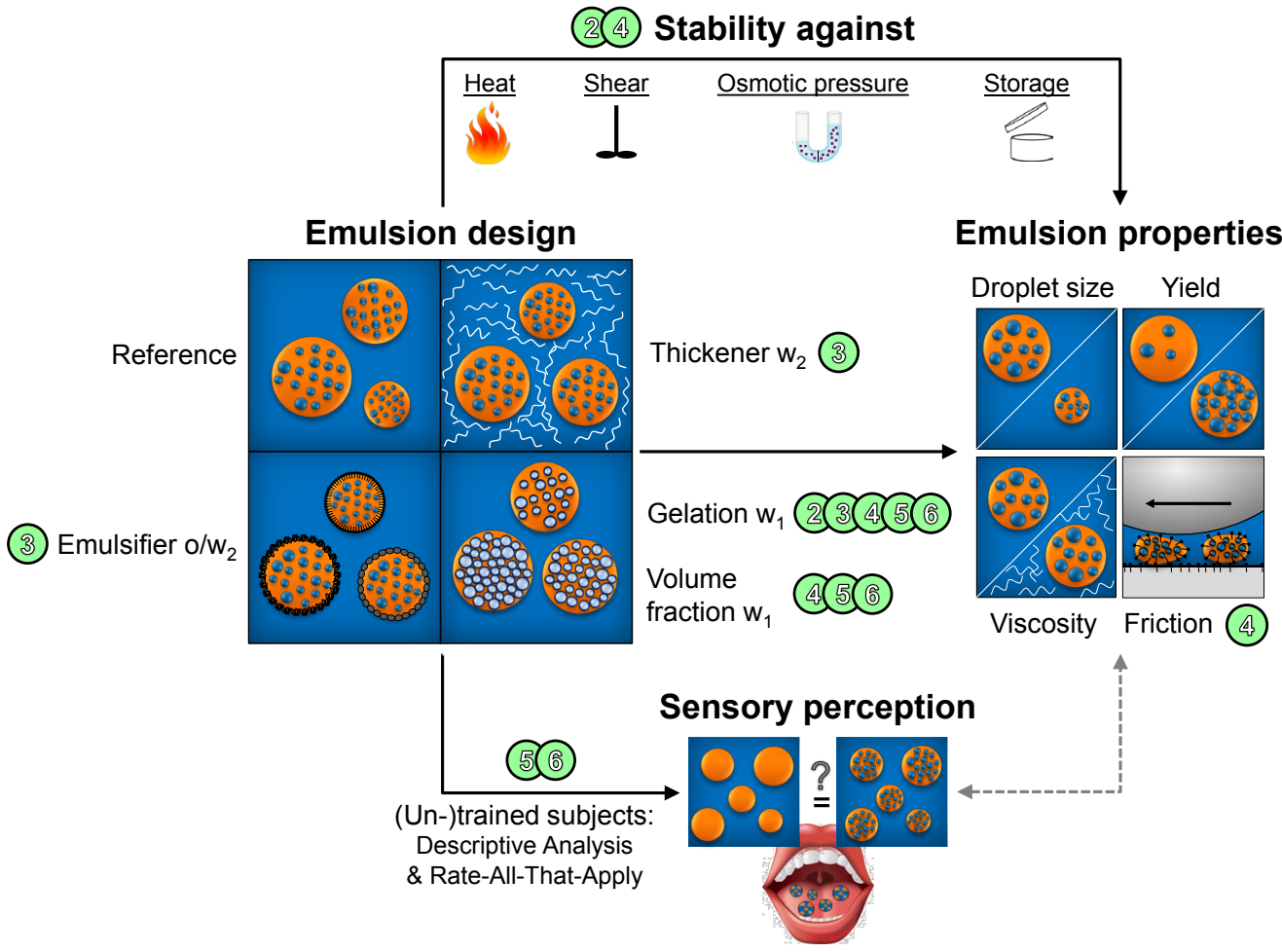

Figure 1.5 Schematic overview of approach of this PhD thesis. Numbers indicate chapters in this thesis. 
In chapter 2, the effect of gelation of the inner water droplets and osmotic pressure imbalance on initial yield, storage and processing stability is investigated. The aim of this chapter was to understand how mechanical properties of the inner water phase influence emulsion characteristics and stability.

In chapter 3, the effect of outer water phase composition on oil droplet size and fat reduction level of double emulsions is studied. In particular, the effect of hydrophilic emulsifier type and the effect of type and concentration of thickener on oil droplet size and fat reduction level is investigated. The aim of this chapter was to understand how the interfacial properties and the viscosity of the continuous phase influence the oil droplet breakup and the fat reduction level.

In chapter 4, lubrication properties of double emulsions are studied. The aim of this chapter was to understand how double emulsion composition and amount of inner water phase influence lubrication properties.

In chapter 5, the sensory perception of double emulsions is investigated by Descriptive sensory Analysis (DA) with a trained sensory panel. The aim of this chapter was to understand how double emulsion composition and fat reduction level influence fatrelated sensory perception. Insights into fat-related sensory perception are crucial for the development of double emulsions as a fat replacement strategy and have not been described previously.

In chapter 6, it is investigated whether an untrained panel using an adapted Rate-AllThat-Apply method provides similar sensory profiles of double emulsions as a trained panel using Descriptive sensory Analysis, as studied in chapter $\mathbf{5}$.

Chapter 7 summarises and integrates the findings of the different chapters of the thesis and discusses the significance of the results and implications towards applications of double emulsions as fat replacers. 


\section{References}

1. World Health Organization. Obesity and overweight. Accessed 7 October 2016. (http://www. who.int/mediacentre/factsheets/fs311/en/)

2. Chung, C. and D.J. McClements, Structure-function relationships in food emulsions: Improving food quality and sensory perception. Food Structure, 2013. 1: p. 106-126.

3. Liu, H., X.M. Xu, and S.D. Guo, Rheological, texture and sensory properties of low-fat mayonnaise with different fat mimetics. LWT - Food Science and Technology, 2007. 40: p. 946-954.

4. Doyen, K., et al., Volatile Release from an Emulsion: Headspace and In-Mouth Studies. Journal of Agricultural and Food Chemistry, 2001. 49: p. 804-810.

5. Bayarri, S., A.J. Taylor, and J. Hort, The role of fat in flavor perception: Effect of partition and viscosity in model emulsions. Journal of Agriculture and Food Chemistry, 2006. 54: p. 8862-8868.

6. Benjamins, J., et al., Partial coalescence as a tool to control sensory perception of emulsions. Food Hydrocolloids, 2009. 23: p. 102-115.

7. Chojnicka-Paszun, A., H.H.J. de Jongh, and C.G. de Kruif, Sensory perception and lubrication properties of milk: Influence of fat content. International Dairy Journal, 2012. 26: p. 15-22.

8. Chung, C., et al., Textural properties of model food sauces: Correlation between simulated mastication and sensory evaluation methods. Food Research International, 2013. 51: p. 310-320.

9. van Aken, G.A., M.H. Vingerhoeds, and R.A. de Wijk, Textural perception of liquid emulsions: Role of oil content, oil viscosity and emulsion viscosity. Food Hydrocolloids, 2011. 25: p. 789-796.

10. van Vliet, T., et al., Colloidal aspects of texture perception. Advances in Colloid and Interface Science, 2009. 150: p. 27-40.

11. Chung, C., et al., Reduced Fat Food Emulsions: Physicochemical, Sensory, and Biological Aspects. Critical Reviews in Food Science and Nutrition, 2016. 56: p. 650-685.

12. Akhtar, M., et al., Factors affecting the perception of creaminess of oil-in-water emulsions. Food Hydrocolloids, 2005. 19: p. 521-526.

13. Liu, K., et al., Fat droplet characteristics affect rheological, tribological and sensory properties of food gels. Food Hydrocolloids, 2015. 44: p. 244-259.

14. van Aken, G.A., M.H. Vingerhoeds, and E.H.A. de Hoog, Food colloids under oral conditions. Current Opinion in Colloid \& Interface Science, 2007. 12: p. 251-262.

15. Le Calvé, B., et al., Fat Perception: How Sensitive are We? Journal of Texture Studies, 2015. 46: p. $200-211$.

16. de Wijk, R.A. and J.F. Prinz, The role of friction in perceived oral texture. Food Quality and Preference, 2005. 16: p. 121-129.

17. de Wijk, R.A., et al., Perceived creaminess of semi-solid foods. Trends in Food Science \& Technology, 2006. 17: p. 412-422.

18. Vingerhoeds, M.H., et al., How emulsion composition and structure affect sensory perception of low-viscosity model emulsions. Food Hydrocolloids, 2008. 22: p. 631-646.

19. Liu, K., Lubrication and perception of foods - Tribological, rheological and sensory properties of particle-filled food systems, PhD thesis. 2016, Wageningen University: Wageningen

20. Clandinin, M.T., et al., Dietary fat: exogenous determination of membrane structure and cell function. The FASEB Journal, 1991. 5: p. 2761-2769.

21. McClements, D.J., Reduced-Fat Foods: The Complex Science of Developing Diet-Based Strategies for Tackling Overweight and Obesity. Advances in Nutrition: An International Review Journal, 2015. 6: p. 338S-352S.

22. Simons, K. and W.L.C. Vaz, Model Systems, Lipid Rafts, and Cell Membranes. Annual Review of Biophysics and Biomolecular Structure, 2004. 33: p. 269-295.

23. Roberts, D.D., et al., Effects of Sucrose, Guar Gum, and Carboxymethylcellulose on the Release of Volatile Flavor Compounds under Dynamic Conditions. Journal of Agricultural and Food Chemistry, 1996. 44: p. 1321-1326.

24. Chung, C., et al., Reduced Fat Food Emulsions: Physicochemical, Sensory, and Biological Aspects. Critical Reviews in Food Science and Nutrition, 2016. 56: p. 650-685.

25. Chung, C., B. Degner, and D.J. McClements, Reduced calorie emulsion-based foods: Protein microparticles and dietary fiber as fat replacers. Food Research International, 2014. 64: p. 664-676.

26. Liu, K., et al., Evidence for ball-bearing mechanism of microparticulated whey protein as fat replacer in liquid and semi-solid multi-component model foods. Food Hydrocolloids, 2016. 52: p. 403-414. 
27. Liu, K., et al., Effect of microparticulated whey protein on sensory properties of liquid and semisolid model foods. Food Hydrocolloids, 2016. 60: p. 186-198.

28. Akoh, C.C., Fat replacers. Food Technology, 1998. 52: p. 47-53.

29. Ciron, C.I.E., et al., Effect of microfluidization of heat-treated milk on rheology and sensory properties of reduced fat yoghurt. Food Hydrocolloids, 2011. 25: p. 1470-1476.

30. Ciron, C.I.E., et al., Modifying the microstructure of low-fat yoghurt by microfluidisation of milk at different pressures to enhance rheological and sensory properties. Food Chemistry, 2012. 130: p. 510-519.

31. Dresselhuis, D.M., et al., Fat retention at the tongue and the role of saliva: Adhesion and spreading of 'proteinpoor' versus 'protein-rich' emulsions. Journal of Colloid and Interface Science, 2008. 321: p. 21-29.

32. Dresselhuis, D.M., et al., The occurrence of in-mouth coalescence of emulsion droplets in relation to perception of fat. Food Hydrocolloids, 2008. 22: p. 1170-1183.

33. McClements, D.J., Advances in fabrication of emulsions with enhanced functionality using structural design principles. Current Opinion in Colloid \& Interface Science, 2012. 17: p. 235-245.

34. Muschiolik, G., Multiple emulsions for food use. Current Opinion in Colloid \& Interface Science, 2007. 12: p. 213-220.

35. Khan, A.Y., et al., Multiple emulsions: An overview. Current Drug Delivery, 2006. 3: p. 429-443.

36. Vandergraaf, S., C. Schroen, and R. Boom, Preparation of double emulsions by membrane emulsification? A review. Journal of Membrane Science, 2005. 251: p. 7-15.

37. Schuch, A., J. Wrenger, and H.P. Schuchmann, Production of W/O/W double emulsions. Part II: Influence of emulsification device on release of water by coalescence. Colloids and Surfaces A: Physicochemical and Engineering Aspects, 2013. 461: p. 344-351

38. Hasenhuettl, G.L. and R.W. Hartel, Food Emulsifiers and Their Applications. $2^{\text {nd }}$ edition, G.L. Hasenhuettl and R.W. Hartel. 2008, New York: Springer.

39. McClements, D.J., Food emulsions: principles, practices, and techniques. 2nd ed. 2005, Boca Raton, FL: CRC Press.

40. McClements, D.J., et al., Structural Design Principles for Delivery of Bioactive Components in Nutraceuticals and Functional Foods. Critical Reviews in Food Science and Nutrition, 2009. 49: p. 577-606.

41. Bahtz, J., et al., Decoupling of Mass Transport Mechanisms in the Stagewise Swelling of Multiple Emulsions. Langmuir, 2015. 31: p. 5265-5273.

42. Mezzenga, R., B.M. Folmer, and E. Hughes, Design of double emulsions by osmotic pressure tailoring. Langmuir, 2004. 20: p. 3574-3582.

43. Cheng, J., et al., Transport of ions through the oil phase of $W(1) / O / W(2)$ double emulsions. J Colloid Interface Sci, 2007. 305: p. 175-82.

44. Wen, L. and K.D. Papadopoulos, Effects of Osmotic Pressure on Water Transport in W(1)/O/W(2) Emulsions. Journal Colloid and Interface Science, 2001. 235: p. 398-404.

45. Balcaen, M., et al., Influence of internal water phase gelation on the shear- and osmotic sensitivity of W/O/W-type double emulsions. Food Hydrocolloids, 2016. 58: p. 356-363.

46. Jiao, J., D.G. Rhodes, and D.J. Burgess, Multiple emulsion stability: pressure balance and interfacial film strength. Journal Colloid and Interface Science, 2002. 250: p. 444-50.

47. Tang, S.Y., M. Sivakumar, and B. Nashiru, Impact of osmotic pressure and gelling in the generation of highly stable single core water-in-oil-in-water (W/O/W) nano multiple emulsions of aspirin

assisted by two-stage ultrasonic cavitational emulsification. Colloids and Surfaces B: Biointerfaces, 2013. 102: p. 653-658.

48. Sapei, L., M.A. Naqvi, and D. Rousseau, Stability and release properties of double emulsions for food applications. Food Hydrocolloids, 2012. 27: p. 316-323.

49. Pawlik, A., P.W. Cox, and I.T. Norton, Food grade duplex emulsions designed and stabilised with different osmotic pressures. Journal of Colloid and Interface Science, 2010. 352: p. 59-67.

50. Iqbal, S., et al., Controlling W/O/W multiple emulsion microstructure by osmotic swelling and internal protein gelation. Food Research International, 2013. 54: p. 1613-1620.

51. Jiménez-Colmenero, F., Potential applications of multiple emulsions in the development of healthy and functional foods. Food Research International, 2013. 52: p. 64-74.

52. Silva, B.F.B., C. Rodríguez-Abreu, and N. Vilanova, Recent advances in multiple emulsions and their application as templates. Current Opinion in Colloid and Interface Science, 2016. 25: p. 98-108.

53. Surh, J., et al., Preparation and characterization of water/oil and water/oil/water emulsions containing biopolymer-gelled water droplets. Journal of Agricultural and Food Chemistry, 2007. 55: p. 175-184. 
54. Su, J., et al., Synergistic effects of polyglycerol ester of polyricinoleic acid and sodium caseinate on the stabilisation of water-oil-water emulsions. Food Hydrocolloids, 2006. 20: p. 261-268.

55. Benichou, A., et al., Formation and characterization of amphiphilic conjugates of whey protein isolate (WPI)/xanthan to improve surface activity. Food Hydrocolloids, 2007. 21: p. 379-391.

56. Benichou, A., A. Aserin, and N. Garti, Double emulsions stabilized with hybrids of natural polymers for entrapment and slow release of active matters. Advances in Colloid and Interface Science 2004. 108-109: p. 29-41.

57. Benichou, A., A. Aserin, and N. Garti, W/O/W double emulsions stabilized with WPI-polysaccharide complexes. Colloids and Surfaces A: Physicochemical and Engineering Aspects, 2007. 294: p. 20-32.

58. Benichou, A., A. Aserin, and N. Garti, Double emulsions stabilized by new molecular recognition hybrids of natural polymers. Polymers for Advanced Technologies, 2002. 13: p. 1019-1031.

59. Benichou, A., A. Aserin, and N. Garti, Protein-polysaccharide interactions for stabilization of food emulsions. Journal of Dispersion Science and Technology, 2002. 23: p. 93-123.

60. Lutz, R., et al., Double emulsions stabilized by a charged complex of modified pectin and whey protein isolate. Colloids and Surfaces B: Biointerfaces, 2009. 72: p. 121-127.

61. Lutz, R., et al., Release of electrolytes from W/O/W double emulsions stabilized by a soluble complex of modified pectin and whey protein isolate. Colloids and Surfaces B: Biointerfaces, 2009. 74: p. 178-185.

62. Mun, S., et al., Effects of enzymatically modified starch on the encapsulation efficiency and stability of water-in-oil-in-water emulsions. Food Chemistry, 2011. 128: p. 266-275.

63. Mun, S., et al., Influence of environmental stresses on the stability of W/O/W emulsions containing enzymatically modified starch. Carbohydrate Polymers, 2013. 92: p. 1503-1511.

64. Perez-Moral, N., S. Watt, and P.J. Wilde, Comparitive Study of the Stability of Multiple Emulsions containing a gelled or aqueous internal phase. Food Hydrocolloids, 2014. 42: p. 215-222.

65. Seddari, S. and N. Moulai-Mostefa, Double Emulsions Stabilized by Xanthan in the Absence of Hydrophilic Surfactant. Journal of Dispersion Science and Technology, 2016. 37: p. 530-535.

66. Florence, A.T. and D. Whitehill, Stabilization of water/oil/water multiple emulsions by polymerization of the aqueous phases. Journal of Pharmacy and Pharmacology, 1982. 34: p. 687-691.

67. Hemar, Y., et al., Encapsulation of Resveratrol Using Water-in-Oil-in-Water Double Emulsions. Food Biophysics, 2010. 5: p. 120-127.

68. Fechner, A., et al., Stability and release properties of double-emulsions stabilised by caseinatedextran conjugates. Food Hydrocolloids, 2007. 21: p. 943-952.

69. Schuch, A., et al., Observations on the influence of different biopolymers on coalescence of inner water droplets in W/O/W (water-in-oil-in-water) double emulsions. Colloids and Surfaces A: Physicochemical and Engineering Aspects, 2014. 475: p. 2-8.

70. Dickinson, E., Double Emulsions Stabilized by Food Biopolymers. Food Biophysics, 2011. 6: p. 1-11.

71. Van de Velde, F. and E. De Hoog, Texture design for creaminess: The role of lubrication, in Food Texture Design and Optimization, Y.L. Dar and J.M. Light, Editors. 2014, John Wiley \& Sons, Ltd.: Chichester, UK. p. 308-320.

72. Kokini, J.L. and E.L. Cussler, Predicting the Texture of Liquid and Melting Semi-Solid Foods. Journal of Food Science, 1983. 48: p. 1221-1225.

73. Le Révérend, B.J.D., et al., Colloidal aspects of eating. Current Opinion in Colloid \& Interface Science, 2010. 15: p. 84-89.

74. Chen, J. and J.R. Stokes, Rheology and tribology: Two distinctive regimes of food texture sensation. Trends in Food Science and Technology, 2012. 25: p. 4-12.

75. Stokes, J.R., M.W. Boehm, and S.K. Baier, Oral processing, texture and mouthfeel: From rheology to tribology and beyond. Current Opinion in Colloid \& Interface Science, 2013. 18: p. 349-359.

76. Cassin, G., E. Heinrich, and H.A. Spikes, The influence of surface roughness on the lubrication properties of adsorbing and non-adsorbing biopolymers. Tribology Letters, 2001. 11: p. 95-102.

77. Joyner, H.S., C.W. Pernell, and C.R. Daubert, Impact of Oil-in-Water Emulsion Composition and Preparation Method on Emulsion Physical Properties and Friction Behaviors. Tribology Letters, 2014. 56: p. 143-160.

78. Malone, M.E., I.A.M. Appelqvist, and I.T. Norton, Oral behaviour of food hydrocolloids and emulsions. Part 1. Lubrication and deposition considerations. Food Hydrocolloids, 2003. 17: p. 763-773.

79. de Wijk, R.A., J.F. Prinz, and A.M. Janssen, Explaining perceived oral texture of starch-based custard desserts from standard and novel instrumental tests. Food Hydrocolloids, 2006. 20: p. 24-34.

80. Schmid, S.R. and W.R.D. Wilson, Lubrication mechanisms for oil-in-water Emulsions. 1996. 
81. de Wijk, R.A. and J.F. Prinz, Mechanisms underlying the role of friction in oral texture. Journal of Texture Studies, 2006. 37: p. 413-427.

82. Kokini, J.L., The physical basis of liquid food texture and texture-taste interactions. Journal of Food Engineering, 1987. 6: p. 51-81.

83. Stone, H., R. Bleibaum, and H.A. Thomas, Sensory Evaluation Practices. 4th ed. 2012, Orlando, FL: Academic Press.

84. Stone, H., et al., Sensory evaluation by Quantitative Descriptive Analysis. Food Technology, 1974. 28: p. $24-29$.

85. Varela, P. and G. Ares, Sensory profiling, the blurred line between sensory and consumer science. A review of novel methods for product characterization. Food Research International, 2012. 48: p. 893-908.

86. Gastón, A. and V. Paula, Comparison of Novel Methodologies for Sensory Characterization, in Novel Techniques in Sensory Characterization and Consumer Profiling. 2014, CRC Press. p. 365- 390.

87. Ares, G., et al., Evaluation of a rating-based variant of check-all-that-apply questions: Rate-all-that-apply (RATA). Food Quality and Preference, 2014. 36: p. 87-95.

88. Reinbach, H.C., et al., Comparison of three sensory profiling methods based on consumer perception: CATA, CATA with intensity and Napping. 2014. 32: p. 160-166.

89. Meyners, M., S.R. Jaeger, and G. Ares, On the analysis of Rate-All-That-Apply (RATA) data. Food Quality and Preference, 2016. 49: p. 1-10. 


\section{-

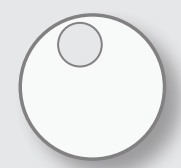

\section{Chapter 2}

\section{Effect of gelation of inner dispersed phase on stability of double $\left(w_{1} / o / w_{2}\right)$ emulsions}
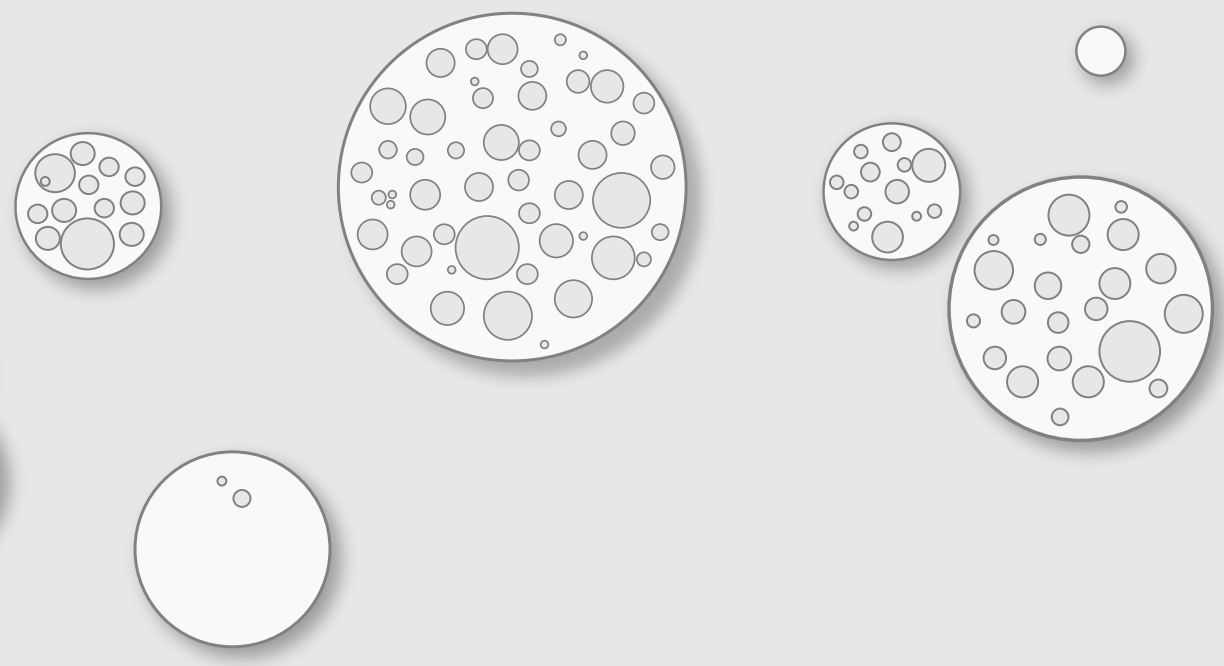

This chapter is based on

AKL Oppermann, M Renssen, A Schuch, M Stieger, E Scholten, Effect of gelation of inner dispersed phase on stability of $\left(\mathrm{w}_{1} / \mathrm{o} / \mathrm{w}_{2}\right)$ multiple emulsions; Food Hydrocolloids (2015), 48, 17-26 


\begin{abstract}
The use of water-in-oil-in-water double $\left(w_{1} / 0 / w_{2}\right)$ emulsions offers a method for the reduction of oil in foods. In this study we investigated the influence of osmotic pressure tailoring and gelation of the inner dispersed $w_{1}$ water droplets on the stability and yield of double emulsions. Yield is defined as the percentage of water retained in the inner dispersed phase $\mathrm{w}_{1}$ after preparation, storage, heat or shear treatment. Differential scanning calorimetry (DSC) was used to determine the yield. Gelation of the inner aqueous phase $\mathrm{w}_{1}$ by gelatin or whey protein isolate increased stability and yield of double emulsions after preparation, storage (after 7 days at $20^{\circ} \mathrm{C}$ ), shear (5 min at $10000 \mathrm{rpm}$ ) and heat treatment $\left(30 \mathrm{~min}\right.$ at $97^{\circ} \mathrm{C}$ ) by $20 \%$ to $50 \%$ compared to reference emulsions. Yield and emulsion stability were correlated to oil droplet sizes and the mechanical properties of the gelled inner dispersed droplets. Yield increased with increasing fracture stress and modulus of gelled $w_{1}$ droplets. We conclude from the present study that stable food-grade double $\left(w_{1} / o / w_{2}\right)$ emulsions can be prepared by gelling the inner dispersed phase. These double emulsions are able to withstand shear and heat treatments. This provides opportunities to use these emulsions as potential fat replacers in foods.
\end{abstract}




\subsection{Introduction}

The use of water-in-oil-in-water double $\left(w_{1} / o / w_{2}\right)$ emulsions offers a potential approach for the reduction of oil content in foods [1]. Double emulsions consist of an inner aqueous phase $\left(\mathrm{w}_{1}\right)$, trapped as small droplets inside larger oil droplets (o), which are subsequently dispersed into another aqueous phase $\left(w_{2}\right)$. By introducing small aqueous droplets in the oil droplets, the amount of oil is decreased while maintaining the same oil droplet surface area. For the potential application of double emulsions as fat replacers in foods, it is crucial that the inner aqueous phase is retained inside the oil droplets during preparation, storage and processing. The preparation procedure and storage stability of double emulsions has been studied extensively [2-9].

The percentage of inner aqueous phase still entrapped in the oil phase at the moment of interest is defined as the yield. For applications of double emulsions as potential fat replacers, it is considered crucial that the yield is as high as possible after preparation, processing and storage. Up to now, mostly indirect measurements like conductivity, photometry, or rheology have been used, which tend to lead to either overestimations or underestimations of the yield and are often not very sensitive to changes in yield [10]. Differential scanning calorimetry (DSC), a method that was used recently to measure the yield of the $w_{1}$-phase, allows the direct determination of the amount of water in the inner water phase of double $\left(w_{1} / o / w_{2}\right)$ emulsions [11]. Schuch and co-workers [10] compared different techniques to determine the yield. They concluded that DSC is the recommended technique to determine also small losses of inner aqueous phase.

In oil-in-water (o/w) emulsions, destabilization via creaming, coalescence and aggregation of the oil droplets can occur. Double emulsions have additional instability issues due to the presence of two interfaces requiring different types of emulsifiers which can affect the yield [12]. Osmotic pressure gradients between the inner $\left(w_{1}\right)$ and the outer aqueous phase $\left(w_{2}\right)$ can lead to diffusion of water molecules, leading to swelling or shrinkage of $w_{1}$ droplets $[13,14]$. Due to a relatively high Laplace pressure of the small inner water droplets, migration of water towards the outer phase is promoted. By adding solutes to the inner dispersed phase, the osmotic pressure gradient can counteract the Laplace pressure and therewith improve stability $[9,13,15,16]$. Although theoretically only the presence of solutes in the inner aqueous phase is necessary to balance the pressure, preliminary research has shown that also a small amount of solute in the outer aqueous phase is needed to create stable double emulsions. 
Diffusion, as well as coalescence of inner aqueous droplets with the outer aqueous phase, changes the amount of water entrapped in the oil droplets. Especially droplet coalescence may be further promoted when emulsions are subjected to intense mechanical stresses, like shearing, centrifugation, or homogenization, which might cause problems for practical applications [17-21]. Another strategy to decrease coalescence of the inner dispersed phase is to convert $w_{1}$ droplets into microgels and thus increase stability [18]. This may be achieved using a biopolymer solution that can be gelled (e.g. by thermal processing) [22, 23]. In terms of proteins, whey protein and gelatin have been used to gel the inner dispersed phase [3, 9, 16, 24-26]. It has been clearly demonstrated by those studies that emulsion stability and/or yield improves through gelation of the $w_{1}$ phase using gelling agents. Very few studies have reported the influence of thermal processing and shear on yield and stability of double emulsions with gelled inner phases [27]. To the best of our knowledge, heat and shear stability of double emulsions containing proteins as gelling agents, and the relation between mechanical properties of gelled inner $w_{1}$ droplets and emulsion properties, is not known.

The aim of the present study was to investigate the stability and yield of double $\left(w_{1} / o / w_{2}\right)$ emulsions containing different inner dispersed phases. We investigated the effect of storage, shear and heat treatment and Laplace-osmotic pressure imbalance on stability and yield. We compared the stability of double emulsions containing a non-gelled or gelled $w_{1}$ phase varying in the type of gelling agent. We hypothesize that the formation of a gel network in the inner aqueous phase minimizes $w_{1}$ dropletdroplet coalescence and reduces the rate of expulsion of these gelled droplets to the external continuous phase, since droplet gelation may modify the energy barrier for coalescence/expulsion. Furthermore, we hypothesize that the initial yield and emulsion stability depends on the mechanical properties of the gelled $w_{1}$ phase. The amount of inner dispersed droplets, expressed as the yield, is determined using differential scanning calorimetry.

\subsection{Materials and methods}

\subsubsection{Materials}

Whey Protein Isolate (WPI, BiPro JE-099-2-420) was obtained from Davisco Foods International Inc. (Le Sueur, MN). The composition of WPI as stated by the manufacturer was $97.7 \%$ protein, $0.3 \%$ fat, $1.8 \%$ ash (dry weight basis) and $4.6 \%$ moisture (wet weight basis). Corrections were made for the impurity of the WPI powder. Polyglycerol 
polyricinoleate (PGPR 4175) was kindly provided by Palsgaard (Juelsminde, Denmark). Commercial sunflower oil was purchased from a local supermarket (Wageningen, the Netherlands). Gelatin (type A, Type 250 PS 30) from Rousselot (Gent, Belgium) was used. $\mathrm{NaCl}$ was obtained from Sigma-Aldrich (purity $\geq 99.5 \%$, Sigma-Aldrich, Steinheim, Germany). For all aqueous solutions, purified water (Milli-Q, 18.2 M $\Omega \mathrm{cm}$ at $25^{\circ} \mathrm{C}$ ) was used.

\subsubsection{Preparation of emulsions}

\subsubsection{Preparation of solutions}

$\mathrm{NaCl}$ solutions were prepared by dissolving $0.3 \mathrm{wt} \%$ or $0.4 \mathrm{wt} \% \mathrm{NaCl}$ in purified water. Gelatin solutions were prepared by heating purified water in a water bath to $60{ }^{\circ} \mathrm{C}$, after which gelatin was added to obtain a 5,10 , or $15 \mathrm{wt} \%$ gelatin solution. $\mathrm{NaCl}(0.3$ or $0.4 \mathrm{wt} \%$ ) was added while stirring. The solution was kept at $60^{\circ} \mathrm{C}$ until the gelatin was completely dissolved. Ten and $15 \mathrm{wt} \% \mathrm{WPI}$ solutions were prepared by slowly adding WPI powder and either 0.3 or $0.4 \mathrm{wt} \% \mathrm{NaCl}$ to purified water while stirring. Stirring was continued in a closed flask for $2 \mathrm{~h}$ at $20^{\circ} \mathrm{C}$ (room temperature) to allow complete dissolution. WPI solutions were stored overnight in a refrigerator at $4{ }^{\circ} \mathrm{C}$. The oil phase was prepared by adding $4 \mathrm{wt} \%$ PGPR to sunflower oil followed by $30 \mathrm{~min}$ stirring at $20^{\circ} \mathrm{C}$. The outer aqueous phase $\mathrm{w}_{2}$ was prepared by slowly adding $1 \mathrm{wt} \% \mathrm{WPI}$ powder and $0.2 \mathrm{wt} \% \mathrm{NaCl}$ to purified water while stirring. Stirring was continued for $2 \mathrm{~h}$ at $20^{\circ} \mathrm{C}$. The solution was stored in the fridge at $4{ }^{\circ} \mathrm{C}$ overnight before use.

\subsubsection{Preparation of $\left(w_{1} / 0\right)$ emulsions}

Primary water-in-oil $\left(w_{1} / 0\right)$ emulsions were prepared by adding $30 \mathrm{wt} \%$ of aqueous phase to $70 \mathrm{wt} \%$ of oil phase in a MC2 stainless steel container of a Waring blender $8011 \mathrm{ES}$ (Stamford, CT), and mixing at $22000 \mathrm{rpm}$ for $45 \mathrm{~s}$. For samples with gelatin in the inner aqueous phase, both the oil phase and the gelatin solution were heated to $60{ }^{\circ} \mathrm{C}$ prior to mixing. For samples with WPI in the inner aqueous phase, a premixing step was added: Thirty weight percent of WPI solution was slowly added to $70 \mathrm{wt} \%$ of oil phase while increasing the mixing speed of a high speed blender (Ultra Turrax T25 with the dispersing tool S25-N 18G, IKA, Staufen, Germany) from $3000 \mathrm{rpm}$ to $8000 \mathrm{rpm}$ within $1 \mathrm{~min}$ and mixed at $8000 \mathrm{rpm}$ for $4 \mathrm{~min}$. The primary emulsion was then transferred to the Waring blender and prepared as described above. After the final mixing step, the $\left(w_{1} / 0\right)$ emulsion with WPI was heated to $80^{\circ} \mathrm{C}$ in a water bath while stirring (with a magnetic stirrer at low speed) and kept at $80^{\circ} \mathrm{C}$ for $20 \mathrm{~min}$ to allow the droplets containing WPI to gel. Samples were subsequently cooled under tap water for at least $15 \mathrm{~min}$. 


\subsubsection{Preparation of double $\left(w_{1} / o / w_{2}\right)$ emulsions}

Double emulsions were prepared by dispersing $30 \mathrm{wt} \%$ of the primary $\left(\mathrm{w}_{1} / \mathrm{o}\right)$ emulsion into $70 \mathrm{wt} \%$ of outer $\mathrm{w}_{2}$ aqueous phase while increasing the mixing speed of the high speed blender (Ultra Turrax T25 with the dispersing tool S25-N 18G, IKA, Staufen, Germany) from $3000 \mathrm{rpm}$ to $7000 \mathrm{rpm}$ within $1 \mathrm{~min}$. Mixing was continued for $4 \mathrm{~min}$ at $7000 \mathrm{rpm}$. All emulsions were prepared and their yield, oil droplet size, and microstructure were characterized at least in duplicate.

\subsubsection{Stability tests}

To assess the shear stability of double emulsions, $50 \mathrm{~g}$ of double emulsions were sheared at $20^{\circ} \mathrm{C}$ using a high speed blender (Ultra Turrax T25 with the dispersing tool S25-N 18G, gap size: 18 mm, IKA, Staufen, Germany) at 8000, 10000 or 12000 rpm for $5 \mathrm{~min}$. Shear stability tests were performed at room temperature. To assess the heat stability of double emulsions, $50 \mathrm{~g}$ of double emulsions were heated in closed flasks to $97^{\circ} \mathrm{C}$ in a water bath, while slowly stirring. After 10 min heating up time, samples were kept at $97^{\circ} \mathrm{C}$ for $30 \mathrm{~min}$ and consequently cooled for $15 \mathrm{~min}$ under tap water. To test storage stability, $50 \mathrm{~g}$ of double emulsions were stored for 7 days at $20^{\circ} \mathrm{C}$ in the dark. After performance of these stability tests, double emulsions were characterized in terms of yield, oil droplet size and microstructure.

\subsubsection{Analyses of emulsions}

\subsubsection{Optical microscopy}

Samples of $\left(w_{1} / 0\right)$ and double $\left(w_{1} / o / w_{2}\right)$ emulsions were analyzed using an optical microscope (Axio Scope 50, Carl Zeiss, Germany) equipped with a CCD camera (Axiocam HRc Carl Zeiss AG, Oberkochen, Germany) and Visio imaging software. To record the microscopic images, a drop of each sample was diluted with a drop of purified water directly on the microscope slide and then gently covered with a cover slip. A drop of immersion oil was used on top of the cover slip to observe the double emulsions using an oil immersion objective (100x magnification). Four pictures were taken for each sample, and a representative one is shown.

\subsubsection{Droplet size measurements}

The droplet sizes of both water droplets in the primary $\left(w_{1} / o\right)$ emulsions and watercontaining oil droplets in the double $\left(w_{1} / 0 / w_{2}\right)$ emulsions were measured by static light scattering with the Mastersizer 2000 (Malvern Instruments, Worcestershire, UK). For primary $\left(w_{1} / 0\right)$ emulsions, the refractive index of the dispersed phase was 
set at 1.33 and the one of the dispersant (sunflower oil with 0.5 wt\% PGPR) at 1.469 . PGPR was used to prevent aggregation of the water droplets. Absorption was set at 0.01 . For double $\left(w_{1} / o / w_{2}\right)$ emulsions, the refractive index of the water-containing oil droplets was set at 1.45 and the one of demineralized water (continuous phase) at 1.33. Absorption was set at 0.01 . The size distribution of each sample was measured three times. Average sizes are reported as $\mathrm{d}_{0,5}$.

\subsubsection{Yield measurements}

DSC measurements (Diamond DSC (Perkin Elmer, Pyris, USA)) were carried out to determine the yield of double emulsions after preparation, storage, heat and shear treatment. The measurement principle relies on the fact that the inner and outer aqueous phases of the double emulsion freeze at different temperatures and can therefore be distinguished. As the heat released during freezing of a substance at a constant cooling rate is directly correlated to its mass, the amount of water in the inner water droplets can be calculated by using the latent heat of freezing. Details of the method can be found elsewhere [11].

The DSC method reported by Schuch and co-workers [11] was used and slightly adapted. A sample mass between 7 and $12 \mathrm{mg}$ was filled in an aluminum pan, hermetically sealed and introduced into the calorimeter. After equilibrating at $+10^{\circ} \mathrm{C}$ for $1 \mathrm{~min}$, the samples were steadily cooled to $-60{ }^{\circ} \mathrm{C}$ at a cooling rate of $5{ }^{\circ} \mathrm{C}$ per minute. For each double $\left(\mathrm{w}_{1} / \mathrm{o} / \mathrm{w}_{2}\right)$ emulsion formulation, the corresponding primary $\left(w_{1} / o\right)$ emulsion was measured as a reference for a yield of $100 \%$. Each formulation was prepared and measured at least twice.

To determine the energy needed for the water in the inner dispersed phase to freeze, the area under the relevant peak in the thermogram was integrated (Pyris data analysis software, Perkin Elmer, Waltham, MA). The yield is defined as the amount of water present in the inner dispersed phase $m_{w 1}(t)$ compared to the amount of inner dispersed phase originally incorporated in the primary emulsion $\left(t_{0}\right)$ and is calculated as follows:

$$
\text { Yield }=\frac{m_{w_{1}}(t)}{m_{w_{1}}\left(t_{0}\right)}=\frac{\frac{\Delta H_{c, w_{1}(\text { wow })}}{\varphi_{w_{1} \text { in wow }}}}{\frac{\Delta H_{c, w_{1}(w o)}}{\varphi_{w_{1} \text { in } w o}}} * 100 \%
$$

with $\mathrm{m}_{\mathrm{w} 1}(\mathrm{t})$ as the mass of inner dispersed water at the moment of interest $t, \mathrm{~m}_{\mathrm{w} 1}\left(\mathrm{t}_{0}\right)$ the initial mass of inner dispersed water, $\Delta \mathrm{H}_{c, w 1 \text { (wow) }}$ the change in enthalpy during the crystallization of the inner aqueous dispersed phase of the double $\left(w_{1} / o / w_{2}\right)$ emulsion in $\mathrm{J} \mathrm{g}^{-1}, \Delta \mathrm{H}_{\mathrm{c}, \mathrm{w} 1(\mathrm{wo})}$ the change in enthalpy during the crystallization of the inner aqueous 
dispersed phase of the primary water-in-oil emulsion, in $\mathrm{J} \mathrm{g}^{-1} . \varphi_{\mathrm{w} 1 \text { in wow }}$ and $\varphi_{\mathrm{w} 1 \text { in wo }}$ are the mass fractions of inner dispersed phase in either the double $\left(w_{1} / 0 / w_{2}\right)$ emulsion or the primary $\left(w_{1} / 0\right)$ emulsion, respectively. Example thermograms of typical DSC measurements of the primary $\left(w_{1} / 0\right)$ and double $\left(w_{1} / 0 / w_{2}\right)$ emulsion are shown in Figure 2.1.

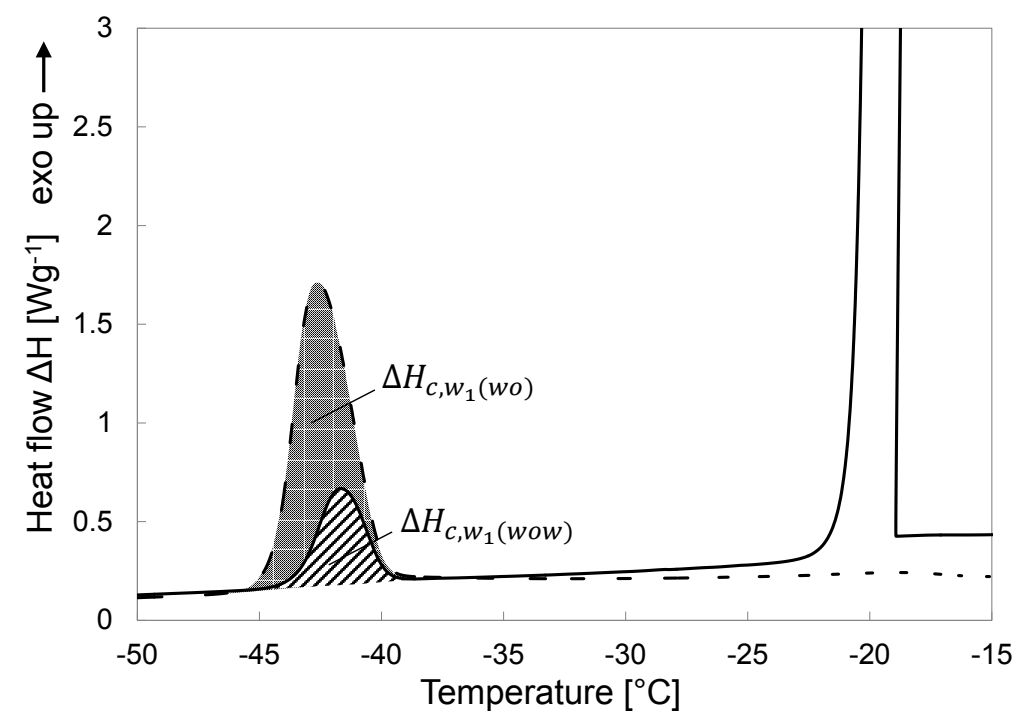

Figure 2.1 Thermograms of typical DSC measurements of a primary $\left(w_{1} / 0\right)$ and a double $\left(w_{1} / o / w_{2}\right)$ emulsion. The latent heat of freezing $\left(\Delta \mathrm{H}_{\mathrm{c}, \mathrm{w} 1(\mathrm{wo})}\right.$ and $\left.\Delta \mathrm{H}_{\mathrm{c}, \mathrm{w} 1(\mathrm{wo})}\right)$ was calculated by analyzing the crystallization peaks of the water droplets.

\subsubsection{Osmotic pressure measurements}

The osmolality of aqueous solutions was measured in triplicate with a freezing point depression Osmometer (Advanced Instruments, Model 3320, Norwood, MA). A sampler was loaded with $20 \mu \mathrm{L}$ of sample and inserted in the sample port of the instrument. Results were expressed in osmolality (Osmol/kg).

\subsubsection{Preparation of gelatin and WPI macroscopic gels for texture analysis}

Gelatin gels were prepared by dissolving 5, 10 or $15 \mathrm{wt} \%$ gelatin with $0.4 \mathrm{wt} \% \mathrm{NaCl}$ in purified water at $60^{\circ} \mathrm{C}$, after which the solution was kept at $60^{\circ} \mathrm{C}$ until dissolved. These solutions were poured in $20 \mathrm{ml}$ syringes, which were previously greased with paraffin oil, and subsequently cooled for $15 \mathrm{~min}$ in tap water. WPI gels were prepared by dissolving 10 or $15 \mathrm{wt} \%$ WPI with $0.4 \mathrm{wt} \% \mathrm{NaCl}$ in purified water at $25^{\circ} \mathrm{C}$ and stirring the solution for $2 \mathrm{~h}$ at $20^{\circ} \mathrm{C}$. After cooling the solutions overnight at $4{ }^{\circ} \mathrm{C}$, the solutions were degassed for $10 \mathrm{~min}$ and poured in $20 \mathrm{ml}$ syringes, which were 
previously greased with paraffin oil. The WPI solution was subsequently heated for $20 \mathrm{~min}$ at $80^{\circ} \mathrm{C}$ in a water bath, after which they were cooled in tap water for $15 \mathrm{~min}$. All gels were kept overnight at $4{ }^{\circ} \mathrm{C}$ and were taken out of the fridge to equilibrate to room temperature three hours before texture characterization.

\subsubsection{Uniaxial compression tests of macroscopic gels}

Uniaxial compression tests of macroscopic gelatin and WPI gels were performed with a Texture Analyzer (TA-XT plus, Stable Micro Systems Ltd., Godalming, UK). A cylindrical gel piece of $20 \mathrm{~mm}$ in height and $17.5 \mathrm{~mm}$ in diameter was prepared. A drop of paraffin oil was added underneath and on top of the sample for lubrication purposes. As a probe, a perpex plate of $75 \mathrm{~mm}$ in diameter was used to perform a uniaxial compression test with a test speed of $1 \mathrm{~mm} / \mathrm{s}$ up to a deformation strain of $90 \%$. True fracture stress, true fracture strain and Young's modulus were obtained from the fracture curves. Eight to twelve replicate measurements were performed for each type of gel and the average value obtained.

\subsubsection{Statistical data analysis}

For statistical data analysis, one-way ANOVA, two-way ANOVA and two-way repeated measures ANOVA were performed with SPSS (IBM SPSS Statistics 21). Where applicable, Tukey's test was performed as a post-hoc test. Data were tested on significant differences between yield and treatment (after preparation, storage, shear or heat treatment) with double emulsion composition as variable and on significant differences between oil droplet sizes and storage time for the double emulsion with $10 \mathrm{wt} \%$ gelatin and $0.4 \mathrm{wt} \% \mathrm{NaCl}$ in $\mathrm{w}_{1}$. Level of significance was set at $\mathrm{p}<0.05$.

\subsection{Results and discussion}

\subsubsection{Storage, heat and shear stability of double $\left(w_{1} / 0 / w_{2}\right)$ emulsions with non-gelled $w_{1}$ phase}

To investigate the stability of the inner water phase remaining inside the oil droplets of a double emulsion, expressed as yield, we performed DSC measurements. A double emulsion was prepared for which the Laplace and osmotic pressure were experimentally adjusted. When neither a signal between the crystallization peaks of the inner $w_{1}$ and outer $w_{2}$ phase nor changes in the freezing enthalpies of $w_{1}$ (i.e. yield) were observed over time, we concluded that the Laplace and osmotic pressure difference of the double emulsion was appropriately adjusted. To balance the pressure and create a stable system, we used $0.4 \mathrm{wt} \% \mathrm{NaCl}$ in $\mathrm{w}_{1}$ and $0.2 \mathrm{wt} \% \mathrm{NaCl}$ and $1 \mathrm{wt} \%$ WPI in $\mathrm{w}_{2}$. The osmotic pressure gradient was found to be $64 \mathrm{mOsm} / \mathrm{kg}$. 
In Figure 2.2, the yield at different conditions (after preparation, after 7 days at $20^{\circ} \mathrm{C}$, after shear and after heat treatment) are displayed for different double $\left(\mathrm{w}_{1} / \mathrm{o} / \mathrm{w}_{2}\right)$ emulsions. The initial yield of the double $\left(w_{1} / 0 / w_{2}\right)$ emulsion without gelling agent (represented by the white bars) was found to be $74.2 \%( \pm 2.4)$. This means that $74.2 \%$ of the initially added amount of inner dispersed phase (30 wt\%) was still present within the oil droplets after the second emulsification step, and $25.8 \%$ of the inner water droplets coalesced with the outer aqueous phase during or directly after the second emulsification step. This corresponds to an oil reduction of $22.5 \%$. Taking into account that coalescence of inner aqueous droplets with the outer aqueous phase depends on the oil droplet size [17] and therefore considerably influences the yield, the yield found in our study is in agreement with other studies [10, 17, 27].

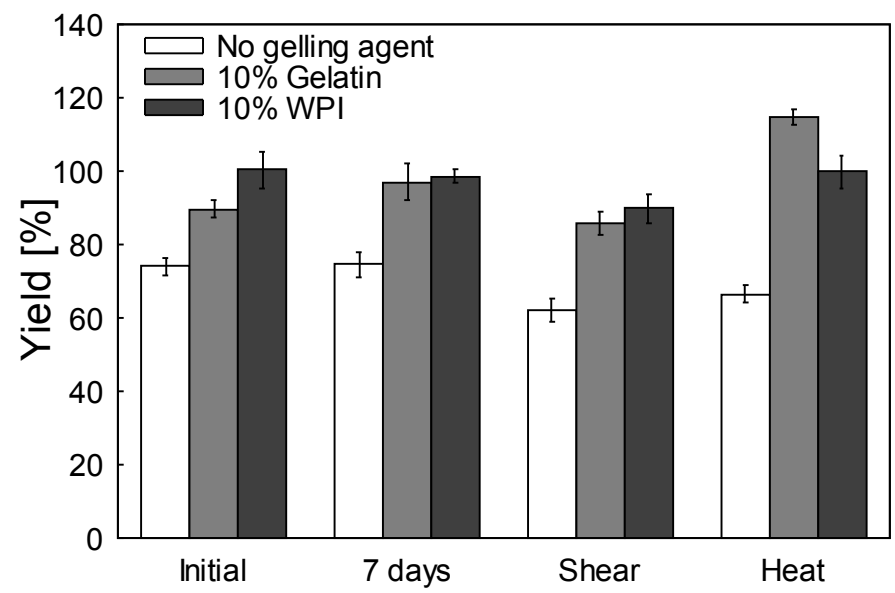

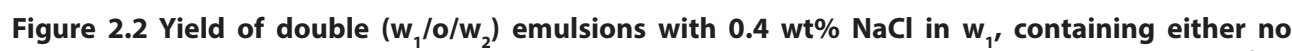
gelling agent (white bars), 10 wt\% gelatin (light grey bars), or 10 wt\% WPI (dark grey bars), after preparation, storage (7 days), shear $(10.000 \mathrm{rpm}, 5 \mathrm{~min})$ and heat $\left(97^{\circ} \mathrm{C}, 30 \mathrm{~min}\right)$ treatment. Error bars indicate the standard deviation of the mean.

To investigate the effect of storage on yield, yield was determined again after seven days of storage in a light-protected space at $20^{\circ} \mathrm{C}$. A change in yield would indicate that the Laplace pressure of the inner dispersed phase and the osmotic pressure difference between the two aqueous phases would not have been matched, and that diffusion of water from one phase to the other would have taken place. We found that yield was constant over time $(74.6 \% \pm 3.3)$. This means that the Laplace pressure within the inner aqueous phase was matched with the osmotic pressure difference between the two aqueous phases. 

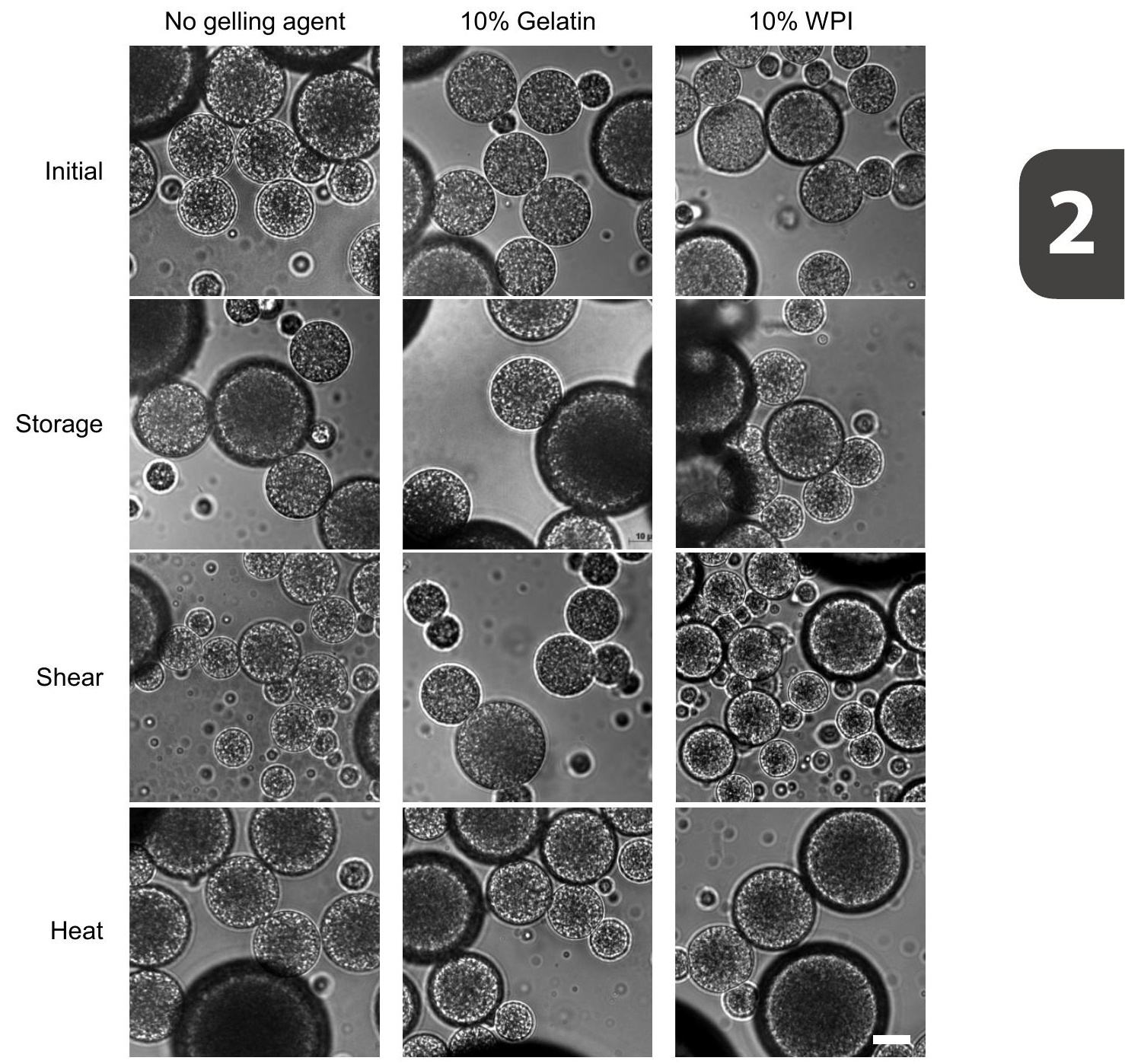

Figure 2.3 Microscopic images of double $\left(w_{1} / 0 / w_{2}\right)$ emulsions with $0.4 \mathbf{w t} \% \mathrm{NaCl}$ in $\mathrm{w}_{1}$ containing either no gelling agent, $10 \mathrm{wt} \%$ gelatin, or $10 \mathrm{wt} \%$ WPI, after preparation, storage (7 days), shear $(10.000 \mathrm{rpm}, 5 \mathrm{~min})$ and heat $\left(97^{\circ} \mathrm{C}, 30 \mathrm{~min}\right)$ treatment. The scale bar of all pictures corresponds to $10 \mu \mathrm{m}$.

To investigate the processing stability of the double $\left(\mathrm{w}_{1} / \mathrm{o} / \mathrm{w}_{2}\right)$ emulsion without gelling the inner dispersed phase, which is from now on referred to as the reference emulsion, the emulsion was either exposed to a shear treatment (Ultra Turrax, $5 \mathrm{~min}$ at $10.000 \mathrm{rpm}$ ) or heat treatment $\left(30 \mathrm{~min}\right.$ holding time at $\left.97^{\circ} \mathrm{C}\right)$. As shown in Figure 2.2, the yield of this reference emulsion decreased from $74.2 \%$ to $62.1 \%$ for the shear-treated sample. Droplet size measurements indicated that the average oil droplet diameter decreased from $72.5 \mu \mathrm{m}( \pm 1.4)$ to $43.8 \mu \mathrm{m}( \pm 1.9)$. This decrease 
in oil droplet size indicates that a combination of oil droplet break up and shearinduced coalescence of the inner water droplets with the outer water phase are the main reasons for the decrease in water content within the oil droplets. The probability of coalescence of the inner dispersed phase with the outer aqueous phase is increased since the contact area between the $\left(w_{1} / 0\right)$ interface and $\left(0 / w_{2}\right)$ interface is increased. Microscopically, this decrease in water content could not be observed (see Figure 2.3). Similar findings have been reported recently by Mun and co-workers [27] who studied a similar double emulsion system.

Heat treatment decreased the yield from $74.2 \%$ to $66.6 \%$ and hence had a smaller influence on the coalescence of inner aqueous phase with the outer aqueous phase. A possible reason for this relatively small decrease in yield is that the osmotic pressure difference is balanced, and that the choice and concentration of both the hydrophilic and hydrophobic emulsifier limits the extent to which coalescence takes place. Oil droplet sizes did not change $(72.5 \mu \mathrm{m}( \pm 1.4)$ initially versus $72.5 \mu \mathrm{m}( \pm 1.8)$ after heating) and did therefore not indicate a difference in stability of these double $\left(w_{1} / o / w_{2}\right)$ emulsions. Similar observations in a double emulsion with $4 \mathrm{wt} \%$ PGPR were made by Mun and co-workers [27]. Although we did not observe changes in the average droplet size $d_{0,5^{\prime}}$ we did notice the appearance of a small amount of larger oil droplet aggregates. This aggregation is most likely an effect of the heating step to $97^{\circ} \mathrm{C}$, a temperature at which WPI denatures and has the ability to aggregate. Hence, when WPI is used as an emulsifier to stabilize the oil droplets, this heating step can cause aggregation of the oil droplets [28]. Additionally, this aggregation of WPI molecules on the $\left(\mathrm{o} / \mathrm{w}_{2}\right)$ interface could have improved the interfacial strength and this might have reduced the loss of the inner droplets through coalescence or diffusion.

Overall, double $\left(w_{1} / 0 / w_{2}\right)$ emulsions with a non-gelled inner aqueous phase had a yield of $74 \%$, equivalent to a fat reduction level of $22.5 \%$. Given the relatively large oil droplet sizes, they showed poor physical stability (i.e. creaming shortly after preparation), but showed reasonable processing stability in terms of shear and heat stability. It should be noted that the $\mathrm{w}_{2}$ phase was not thickened by addition of a thickener to minimize creaming, since this may influence emulsion properties by interactions of the thickener with the hydrophilic emulsifier. 


\subsubsection{Storage, heat and shear stability of double $\left(w_{1} / 0 / w_{2}\right)$ emulsions with gelled $w_{1}$ phase}

To increase the initial yield and to consequently study the processing stability of double $\left(w_{1} / o / w_{2}\right)$ emulsions with gelled $w_{1}$ phase, we used gelatin and whey protein isolate (WPI) to gel the inner aqueous phase.

The addition of gelatin and WPI may change the osmotic pressure of the inner droplets, thereby producing an imbalance in the system. To compensate for this potential change, we investigated whether changing the amount of salt was necessary. We measured the osmotic pressure of gelatin and WPI solutions and adapted in preliminary experiments the $\mathrm{NaCl}$ concentration in the inner aqueous phase to match the osmotic pressure difference between the inner and outer aqueous phase to the osmotic pressure difference described in section 2.3.1 (results not shown). However, after adaptation, we found very low and time-dependent yields. When the original $\mathrm{NaCl}$ concentration was used, we obtained the highest stability. Apparently, the presence of the gel does not affect the osmotic pressure in the same manner as a solution would. As the osmotic pressure is determined by the number of dissolved molecules (solutes), aggregation of these molecules will lead to a decrease in osmotic pressure [29]. A gel would therefore contribute very little to the osmotic pressure as the network could be seen as one molecule. Therefore, we concluded that the contribution to the osmotic pressure of a gelatin or WPI gel can be neglected and we chose to use the same $\mathrm{NaCl}$ concentration in the inner aqueous phase as used in the reference emulsion.

Double $\left(w_{1} / 0 / w_{2}\right)$ emulsions with gelling agents were prepared with the same twostep emulsification process with adaptations to gel the inner aqueous phase. The resulting water and oil droplet sizes were comparable to those of the double $\left(w_{1} / 0 / w_{2}\right)$ emulsion without additional gelling agents (see Table 2.1). As shown in Figure 2.2, initial yields were significantly increased. The yield was $89.6 \%( \pm 2.3)$ for the double $\left(w_{1} / 0 / w_{2}\right)$ emulsion containing $10 \mathrm{wt} \%$ gelatin in $w_{1}$ (represented by the light grey bars) and $100.4 \%( \pm 5.0)$ for the double $\left(w_{1} / 0 / w_{2}\right)$ emulsion containing $10 \mathrm{wt} \% \mathrm{WPI}$ in $\mathrm{w}_{1}$ (represented by the dark grey bars). These values are higher than the value found for the reference emulsion ( $74.2 \%$ ) indicating that the addition of gelling agents increases the yield. Muschiolik and co-workers [30] and Sapei and co-workers [9] also found that the addition of gelatin increased the yield. Surh and co-workers [3] found similar yields, measured by photometry, with their double emulsion system containing $15 \mathrm{wt} \% \mathrm{WPI}$. Unlike the differences we found in our study they did not observe any differences between double emulsions with and without WPI and 
concluded that gelling the inner aqueous phase would not help increasing the yield of the double emulsion. The difference in their and our study lies in the oil droplet sizes and the method of yield determination. Surh and co-workers found oil droplet sizes of double emulsions of 1 to $10 \mu \mathrm{m}$ with yields above $98 \%$. These droplet sizes are much smaller than the ones in our study, and they determined the yield with photometry. However, as discussed recently by Schuch and co-workers [10], photometry might overestimate the amount of entrapped water and therefore the yield found by Surh and co-workers may be overestimated for both gelled and non-gelled emulsions. A comparison between emulsions with gelled or non-gelled inner dispersed phase may therefore be difficult based on this technique.

Table 2.1 Water and oil droplet sizes of $\left(w_{1} / 0\right)$ and $\left(w_{1} / 0 / w_{2}\right)$ emulsions with gelled/non-gelled $w_{1}$ phase with $0.4 \mathrm{wt} \% \mathrm{NaCl}$ in $\mathrm{w}_{1}$ after preparation, storage, shear and heat.

\begin{tabular}{|c|c|c|c|c|c|c|}
\hline \multirow{2}{*}{$\begin{array}{l}\text { Composition } w_{1} \\
\text { Concentration } \\
\text { gelling agent (wt\%) }\end{array}$} & \multirow{2}{*}{$\begin{array}{c}0.4 \% \mathrm{NaCl} \\
0\end{array}$} & \multicolumn{3}{|c|}{ Gelatin $+0.4 \% \mathrm{NaCl}$} & \multicolumn{2}{|c|}{$\mathrm{WPI}+0.4 \% \mathrm{NaCl}$} \\
\hline & & 5 & 10 & 15 & 10 & 15 \\
\hline \multicolumn{7}{|c|}{ Water droplets } \\
\hline $\mathrm{d}_{0,5}(\mathrm{~nm})$ & $175 \pm 0.01$ & $220 \pm 0.01$ & $175 \pm 0.07$ & $277 \pm 0.02$ & $143 \pm 0.02$ & $160 \pm 0.01$ \\
\hline \multicolumn{7}{|c|}{ Oil droplets } \\
\hline $\mathrm{d}_{0.5}$, initial $(\mu \mathrm{m})$ & $72.5 \pm 1.4$ & $68.3 \pm 2.7$ & $72.6 \pm 2.7$ & $73.2 \pm 1.0$ & $67.7 \pm 0.6$ & $62.0 \pm 3.3$ \\
\hline $\mathrm{d}_{0.5}, 7$ days $(\mu \mathrm{m})$ & $72.5 \pm 1.8$ & $-*$ & $73.2 \pm 1.2$ & $-*$ & $70.6 \pm 0.5$ & $-*$ \\
\hline $\mathrm{d}_{0.5}$, shear $(\mu \mathrm{m})$ & $43.8 \pm 1.9$ & $47.2 \pm 2.1$ & $49.7 \pm 5.7$ & $49.6 \pm 3.4$ & $37.3 \pm 2.4$ & $35.8 \pm 0.4$ \\
\hline $\mathrm{d}_{0.5}$, heat $(\mu \mathrm{m})$ & $74.0 \pm 2.0$ & $68.9 \pm 2.3$ & $77.8 \pm 3.0$ & $76.4 \pm 1.3$ & $72.8 \pm 1.6$ & $65.0 \pm 0.8$ \\
\hline
\end{tabular}

* Data not available

We hypothesized that the formation of a gel network in the inner aqueous phase prevents $\mathrm{w}_{1}$ droplet-droplet coalescence and therefore increases the yield. It is thought that the use of gelling agents in the inner aqueous phase leads to the formation of a complex interfacial structure. This complex interfacial structure is believed to be more rigid, which might prevent coalescence of inner dispersed droplets [31]. Coalescence of inner dispersed droplets would lead to an increase in inner dispersed droplet size and a shift in the droplet size distribution. As Schuch and co-workers [11] have shown, the DSC thermogram also gives some qualitative information about the droplet size distribution of the inner dispersed phase. A change in inner dispersed droplet size distribution would influence the onset temperature and freezing curve of the inner dispersed phase. However, no obvious shift in the freezing curve was observed for the reference emulsion indicating that no inner droplet-droplet coalescence occurred, possibly due to relatively highly covered surfaces $\left(w_{1} / 0\right)$ by PGPR. Therefore, gelling of the inner dispersed droplets does not seem to have influenced the occurrence of inner 
$w_{1}$ droplet-droplet coalescence. As oil droplet sizes of double $\left(w_{1} / 0 / w_{2}\right)$ emulsions with gelled and non-gelled $w_{1}$ phase were similar, $w_{1}-w_{2}$ coalescence (at constant mass fraction of $w_{1}$ in oil) seems to influence the yield more than $w_{1}$ droplet-droplet coalescence [32]. Therefore, the difference in yields of double $\left(w_{1} / o / w_{2}\right)$ emulsions with gelled and non-gelled $w_{1}$ phase might lie in the expulsion mechanism. Expulsion of inner gelled droplets into the outer aqueous phase is less likely as the gelled droplet has to penetrate the $\left(o / w_{2}\right)$ interface before it is expelled. This requires more energy compared to the coalescence of inner (non-gelled) water droplets with the outer water phase and is therefore unfavorable. Non-gelled inner aqueous droplets already coalesce with the outer aqueous phase when the oil film separating the two aqueous phases ruptures over a very small region. This rupture leads to immediate merge of water from both aqueous phases. Therefore, changing the interfacial properties by gelling the inner dispersed droplets would reduce the loss of inner dispersed droplets from the oil droplets.

To study the yield over time, emulsions were stored at $20^{\circ} \mathrm{C}$ in a light-protected space for seven days. As shown in Figure 2.2, the yield of the double emulsion containing WPI is stable over seven days, indicating a match of Laplace pressure in the inner dispersed phase and osmotic pressure difference between both aqueous phases. With regard to the emulsion containing $10 \%$ gelatin, however, we observed a small increase in yield from $89.6 \%( \pm 2.3)$ to $97.1 \%( \pm 4.9)$ over seven days. This increase may be explained by the ability of gelatin gels to swell over time due to the flexible fine-stranded network. Indeed, in a swelling experiment we found that macroscopic gelatin gels increase in mass by $43 \%$ within 24 hours when gel cubes were placed directly in purified water (results not shown). The ability of inner dispersed droplets to swell within oil droplets may have caused the slight increase in yield over time. This increase in the volume of the aqueous droplets is, however, not large enough to cause a significant change in oil droplet size (see Table 2.1). Such a change in yield does thus not necessarily result in changes in oil droplet size, or changes in the microstructure as displayed in Figure 2.3.

To study the processing stability of double $\left(w_{1} / 0 / w_{2}\right)$ emulsions containing either gelatin or WPI gelled inner dispersed phase, the emulsions underwent a shear treatment (5 min at $10.000 \mathrm{rpm}$ in an Ultra Turrax) or heat treatment $\left(30 \mathrm{~min}\right.$ at $97^{\circ} \mathrm{C}$ in a water bath). The yield of the sheared emulsions containing $10 \%$ gelatin in $\mathrm{w}_{1}$ decreased slightly from $89.6 \%( \pm 2.3)$ to $85.9 \%( \pm 3.0)$, while the yield of the emulsion containing $10 \mathrm{wt} \% \mathrm{WPI}$ in $\mathrm{w}_{1}$ decreased from $100.4 \%( \pm 5.0)$ to $90.0 \%( \pm 4.0)$ (see Figure 2.2). The change in yield is thus different between the type of gelling agents 
used. The double emulsion containing gelatin lost the least water (change of yield of $4 \%$ ) from the oil droplets and was most shear stable, while the double emulsion containing WPI lost the most of the initial water (10\%) after emulsion preparation. However, since the initial yield of the WPI-containing emulsions was larger, the emulsions with WPI still contained more entrapped water after shear treatment compared to the emulsions with gelatin. The changes in yield are also reflected in the oil droplet sizes. All emulsions decreased in droplet size (see Table 2.1), of which the emulsion containing WPI in the inner aqueous phase decreased most (from $67.7 \mu \mathrm{m}$ \pm 0.6 initially to $37.3 \mu \mathrm{m} \pm 2.4$ after shear), compared to the emulsion containing gelatin (from $72.6 \mu \mathrm{m} \pm 2.7$ initially to $49.7 \mu \mathrm{m} \pm 5.7$ after shear). This decrease in oil droplet size can be explained to a large extent by oil droplet break-up and to a negligible extent by size reduction due to loss of inner dispersed phase. The absolute change in yield was larger for the emulsion containing WPI, which might be explained by the difference in the mechanical properties of the gels made from gelatin and WPI and differences in the microstructure of the gels.

The effect of heat treatment on the yield of our double emulsions containing gelatin or WPI is also shown in Figure 2.2. On one hand, double emulsions containing a gelled inner dispersed phase with $10 \mathrm{wt} \% \mathrm{WPI}$ (and $0.4 \mathrm{wt} \% \mathrm{NaCl}$ ) did not show any change in yield before and after the heat treatment $(100.4 \% \pm 5.0$ initially, versus $99.9 \% \pm 4.6$ after heating). This was expected as whey protein isolate forms thermo irreversible gels, being the reason why coalescence and hence yield of emulsions containing these microgels should not be affected during the heat treatment. On the other hand, the yield of double emulsions containing a gelled inner dispersed phase with $10 \mathrm{wt} \%$ gelatin (and $0.4 \mathrm{wt} \% \mathrm{NaCl}$ ) increased from $89.6 \% \pm 2.3$ initially to $114.6 \% \pm 2.1$ after heating. This increase in yield was also detected in droplet size measurements ( $72.6 \mu \mathrm{m} \pm 2.7$ initially versus $77.8 \mu \mathrm{m} \pm 3.0$ after heating). We suggest that the reason for this increase in yield lies in the thermal reversibility of the gelation of gelatin microgels. Gelatin gels melt upon heating above $35{ }^{\circ} \mathrm{C}$. In a liquid state, gelatin is present in the form of individual gelatin molecules, increasing the osmotic pressure as compared to the gelatin gel (which can be considered as one molecule). This increase in osmotic pressure of the inner aqueous droplets acts on the imbalance in osmotic pressure, which in turn leads to a water flux from $\mathrm{w}_{2}$ to $\mathrm{w}_{1}$, increasing the size of the inner water droplets. We were not able to confirm the increased osmotic pressure at elevated temperatures, as our measurement technique to determine the osmotic pressure was based on determining the freezing point depression. Once the double emulsions are cooled down again, liquid gelatin droplets re-form into a fine-stranded 
gel network, entrapping a higher amount of water compared to before resulting in an increase of yield. In another set of experiments, in which we varied the amount of gelatin $(5,10$ and $15 \mathrm{wt} \%)$, we found that the gelatin concentration affects the initial yield and increase in yield after heating to a large extent (Figure 2.4). The higher the gelatin concentration, the more gelatin molecules are present in the heated solution and therefore a larger change in the osmotic pressure difference between $w_{1}$ and $w_{2}$. Therefore, the yield of emulsions with 10 and $15 \mathrm{wt} \%$ gelatin increased significantly more compared to the emulsion containing only $5 \mathrm{wt} \%$ gelatin. The relative increases in yield upon heat treatment were about $28 \%$ for both 10 and $15 \mathrm{wt} \%$ gelatin, and approximately $11 \%$ for 5 wt $\%$ gelatin.

Overall, double $\left(w_{1} / 0 / w_{2}\right)$ emulsions with a gelled inner aqueous phase showed significantly increased yields and excellent processing stability in terms of shear and heat stability, compared to the reference double emulsion.

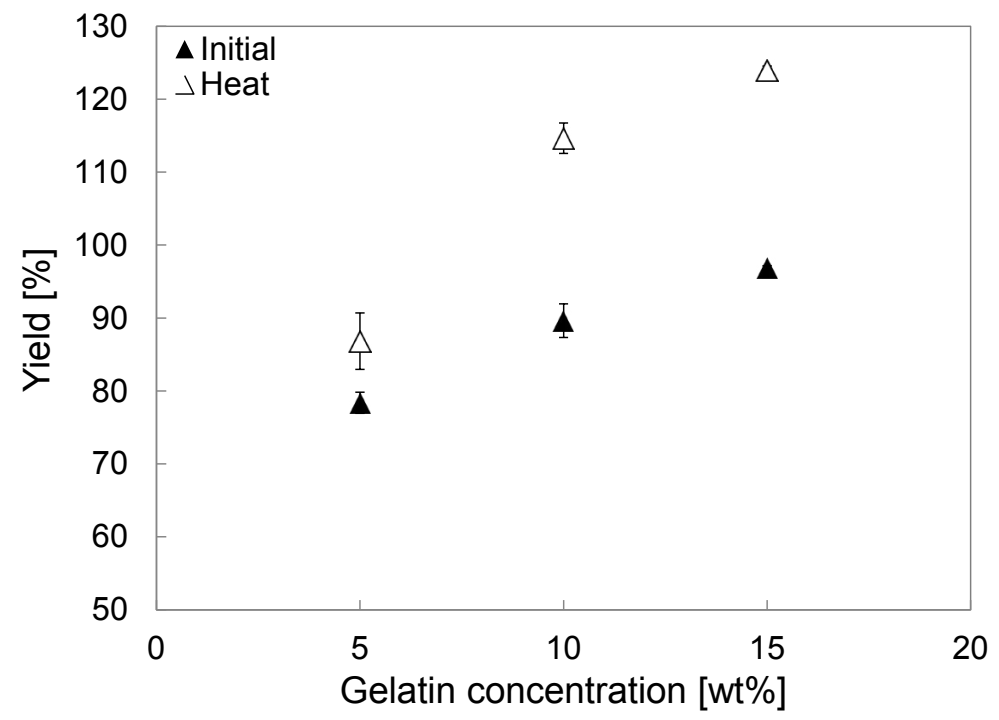

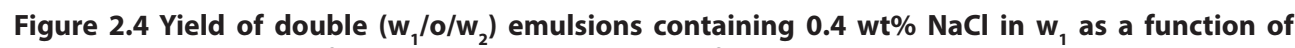
gelatin concentration, after preparation (initial) and after heat treatment $\left(97^{\circ} \mathrm{C}, 30 \mathrm{~min}\right)$. Error bars indicate the standard deviation of the mean.

\subsubsection{Effect of osmotic pressure tailoring on storage, heat and shear stability of double emulsions with gelled/non-gelled $w_{1}$ phase}

As described in section 2.3.1, an osmotic pressure difference that counterbalances the Laplace pressure is essential for a stable double $\left(w_{1} / o / w_{2}\right)$ emulsion system. To study the influence of gelling the inner dispersed phase on the yield and the stability of an emulsion system that is not balanced in osmotic and Laplace pressure, we changed 
the osmotic pressure difference between $w_{1}$ and $w_{2}$ by varying the concentration of $\mathrm{NaCl}$ in the inner aqueous phase $\mathrm{w}_{1}$, from 0.4 to $0.3 \mathrm{wt} \%$. By decreasing the salt concentration in $w_{1}$, a pressure imbalance leads to a water flux from $w_{1}$ to $w_{2}$. In this case, we would therefore expect a decrease in inner water droplet size and a subsequent decrease in oil droplet size over time $\left(t_{0}\right.$ versus $\left.t_{7}\right)$. We hypothesize that by gelling the inner droplet, the water diffusion can be partially prevented due to the entrapment of water. The results for the yield of gelled and non-gelled double emulsions with $0.3 w t \% ~ N a C l$ in $w_{1}$ are shown in Figure 2.5. Yields of the double emulsions were measured four hours after preparation. As the osmotic pressure difference was not in balance with the Laplace pressure of the inner dispersed droplets, the emulsions were not yet in equilibrium at that moment. This might explain the relatively large standard deviations within this set of samples and the small difference in yield after seven days of storage for some of the samples. However, also in this series of samples, we observe that the addition of gelling agents to $w_{1}$ significantly increased the initial yield, as well as its storage and processing stability, although none of the samples reached the yield of its counterpart when the osmotic pressure difference was appropriately adjusted.

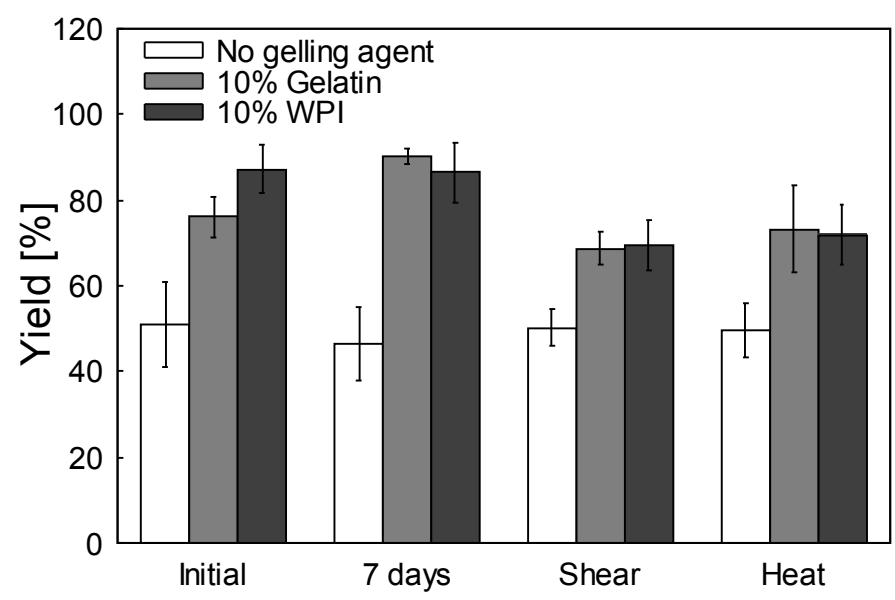

Figure 2.5 Yield of $\left(w_{1} / 0 / w_{2}\right)$ double emulsions with $0.3 \mathrm{wt} \% \mathrm{NaCl}$ in $\mathrm{w}_{1}$ containing either no gelling agent, $10 \mathrm{wt} \%$ gelatin, or $10 \mathrm{wt} \% \mathrm{WPI}$, after preparation, storage ( 7 days), shear ( $10.000 \mathrm{rpm}, 5 \mathrm{~min}$ ) and heat $\left(97^{\circ} \mathrm{C}, 30 \mathrm{~min}\right)$ treatment. Error bars indicate the standard deviation of the mean.

These results show that changing the concentration of $\mathrm{NaCl}$ in $\mathrm{w}_{1}$ from $0.4 \mathrm{wt} \%$ to $0.3 \mathrm{wt} \%$ (and therefore reducing the osmotic pressure gradient between $w_{1}$ and $w_{2}$ ) leads to smaller changes in yield when the inner water phase $w_{1}$ was gelled compared to a non-gelled $w_{1}$ phase. Nevertheless, to have the highest yield and the largest stability, one should always aim to balance Laplace and osmotic pressure differences in a double $\left(w_{1} / o / w_{2}\right)$ emulsion or more complex (food) systems. 


\subsubsection{Relation between mechanical properties of inner $w_{1}$ droplets, oil droplet size and yield of double $\left(w_{1} / 0 / w_{2}\right)$ emulsions}

In the previous sections, we studied the influence of gelation of the inner dispersed phase on the stability of double $\left(w_{1} / o / w_{2}\right)$ emulsions. The results show that the yield for double $\left(w_{1} / o / w_{2}\right)$ emulsions with gelled inner dispersed phase depended on the type of gelling agent (gelatin, WPI) used. This may be related to the mechanical properties of the gels and/or the microstructure of the gels. To compare the properties of the WPI and the gelatin inner $\mathrm{w}_{1}$ droplets, we analyzed the mechanical properties of macroscopic gels as an indication for their mechanical properties on a microscopic scale. As shown by Saglam and co-workers [33], macroscopic WPI gels and WPI microgels were comparable in gel microstructure. We assume that the mechanical properties of macroscopic gels and microgels are also comparable. The network of gelatin is fine-stranded, and the network of WPI coarse-stranded. In the case of $0.4 \mathrm{wt} \% \mathrm{NaCl}$ and $10 \mathrm{wt} \%$ gelling agent, the Young's moduli were found to be similar: $16.8 \mathrm{kPa}( \pm 0.4)$ for gelatin and $19.0 \mathrm{kPa}( \pm 0.3)$ for WPI (see Table 2.2). For the true fracture stress, we observed a larger difference between the gel types $(53.9 \mathrm{kPa} \pm 5.5$ for gelatin, and $72.6 \mathrm{kPa} \pm 8.9$ for WPI). To investigate the effect of the mechanical properties of the gels on the stability and the yield of the emulsions, we varied the mechanical properties by varying the concentration of gelling agent. With an increasing concentration of gelatin or WPI, the Young's moduli and fracture stresses increased, as can be seen in Table 2.2. The relation between true fracture stress and initial yield of the double emulsion is shown in Figure 2.6.

Table 2.2 True facture stress, true fracture strain, and Young's moduli of macroscopic gels with 0.4 wt $\% ~ N a C l$ in $w_{1}$.

\begin{tabular}{llllll}
\hline & \multicolumn{3}{c}{ Gelatin } & \multicolumn{2}{c}{ WPI } \\
\hline Concentration & $5 \%$ & $10 \%$ & $15 \%$ & $10 \%$ & $15 \%$ \\
Young's Modulus $(\mathrm{kPa})$ & $4.4 \pm 0.1$ & $16.8 \pm 0.4$ & $34.3 \pm 1.5$ & $19.0 \pm 0.3$ & $134.2 \pm 1.7$ \\
True fracture stress $(\mathrm{kPa})$ & $17.5 \pm 3.4$ & $53.9 \pm 5.5$ & $102.6 \pm 10.1$ & $72.6 \pm 8.9$ & $139.0 \pm 5.8$ \\
True fracture strain (-) & $1.1 \pm 0.1$ & $1.43 \pm 0.1$ & $1.61 \pm 0.1$ & $1.59 \pm 0.1$ & $1.3 \pm 0.1$ \\
\hline
\end{tabular}

As shown in Figure 2.6, the yield of double $\left(w_{1} / 0 / w_{2}\right)$ emulsions containing gelatin increases steadily with increasing concentration from $78.4 \%( \pm 1.5)$ for $5 \mathrm{wt} \%$ gelatin, to $89.6 \%$ ( \pm 2.3$)$ for $10 \mathrm{wt} \%$ gelatin, to $96.9 \%( \pm 0.3)$ for $15 \mathrm{wt} \%$ gelatin. Likewise, the yield of double $\left(\mathrm{w}_{1} / \mathrm{o} / \mathrm{w}_{2}\right)$ emulsions containing WPI, increased from $100.4 \%( \pm 5.0)$ for 10 wt $\%$ WPI to $110.9 \%$ ( \pm 1.8$)$ for $15 w t \%$ WPI. Five wt $\%$ WPI was not included as this concentration does not yet lead to the formation of a gel. Yet, it is unclear if initial yields above $100 \%$ are realistic, as it means that during the second emulsification step, diffusion of water from $w_{2}$ to $w_{1}$ occurred or that additional water would be 


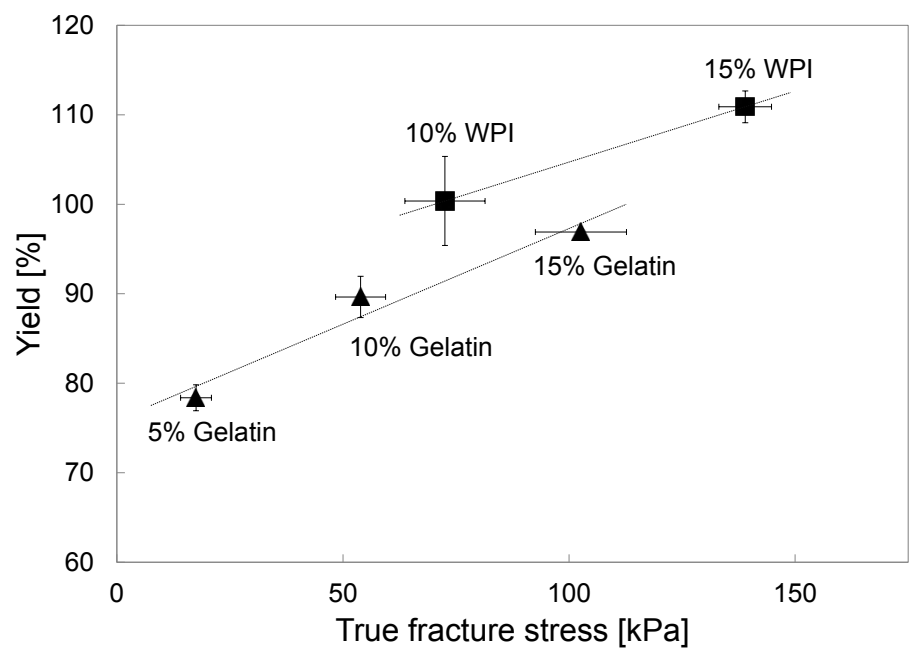

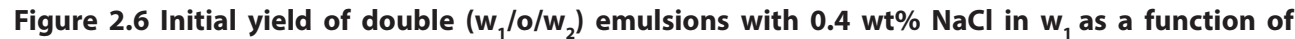
fracture stress of the inner dispersed droplets. The fracture stress was altered by changing the concentration of either WPI (squares) or gelatin (triangles). Error bars indicate the standard deviation of the mean. The dotted lines are added to guide the eye.

entrapped within the oil droplets. This is theoretically possible, since the system contains an excess of PGPR to create additional new inner dispersed droplets without gelling agent [15].

There seems to be a correlation between yield and fracture stress for both systems. The increased fracture stress for WPI droplets may partly explain the higher initial yields for double $\left(\mathrm{w}_{1} / \mathrm{o} / \mathrm{w}_{2}\right)$ emulsions with WPI (yield $=100.4 \%$ ), compared to emulsions with gelatin in $\mathrm{w}_{1}$ (yield $=89.6 \%$ ) in the case of $10 \mathrm{wt} \%$ gelling agent. Although the results show that there is most likely a relation between yield and fracture stress, this relation is not the same for both gelling agents. This indicates that the yield obtained also depends on the type of gelling agent used. We have only reported the results of fracture stress of gelled $w_{1}$ droplets, but we see similar results for relationships between Young's modulus of the gelled $w_{1}$ droplets and yield.

The effect of the type of gelling agent was also observed when the yield was measured as a function of fracture stress of the droplets before and after an additional shear treatment. Figure $\mathbf{2 . 7}$ shows this relation for the initial emulsions (closed symbols), and after a shear treatment with a high speed blender (5 $\mathrm{min}, 10000 \mathrm{rpm}$ ) represented by the open symbols. In the case of emulsions with WPI (Figure 2.7 A), we observe a large difference in the yield (from $100.4 \%$ to $90.0 \%( \pm 4.0)$ for $10 \mathrm{wt} \% \mathrm{WPI}$ and from $110.9 \%$ to $100.7 \%( \pm 3.1)$ for $15 \mathrm{wt} \% \mathrm{WPI})$, whereas in the case of gelatin (Figure 2.7 B), the yield changed by a much smaller extent (from $78.4 \%$ to $76.0 \%$ ( \pm 0.5$)$ for $5 \mathrm{wt} \%$ 

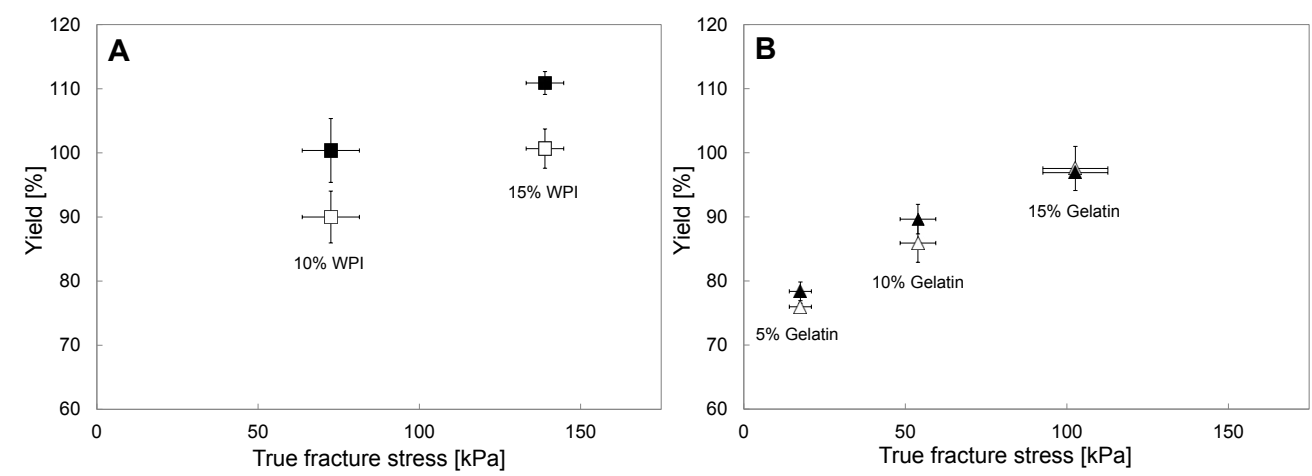

Figure 2.7 Yield of double $\left(\mathrm{w}_{1} / \mathrm{o} / \mathrm{w}_{2}\right)$ emulsions with $0.4 \mathrm{wt} \% \mathrm{NaCl}$ in $\mathrm{w}_{1}$ plotted versus the fracture stress of the inner dispersed droplets. The fracture stress was altered by changing the concentration of either WPI (A, squares) or gelatin (B, triangles). Filled symbols represent the initial yield, open symbols represent the yield after shear treatment $(10.000 \mathrm{rpm}, 5 \mathrm{~min})$. Error bars indicate the standard deviation of the mean.

gelatin, from $89.6 \%$ to $85.9 \%$ ( \pm 3.0 ) for 10 wt $\%$ gelatin, and from $96.9 \%$ to $97.5 \%$ ( \pm 3.4) for 15 wt $\%$ gelatin).

The difference in the yield may not be related only to the type of the gelling agent used, but also to the oil droplet sizes of the emulsions, as droplet size and yield have been shown to be related [17]. Both the type of gelling agent and the fracture stress of the gel may have an effect on the oil droplet size. Figure $\mathbf{2 . 8}$ shows the oil droplet size as a function of fracture stress of the inner dispersed droplets gelled with either WPI (squares) or gelatin (triangles) for the initial double emulsion (closed symbols) and after shear treatment (open symbols). Oil droplet size seems to be influenced not only

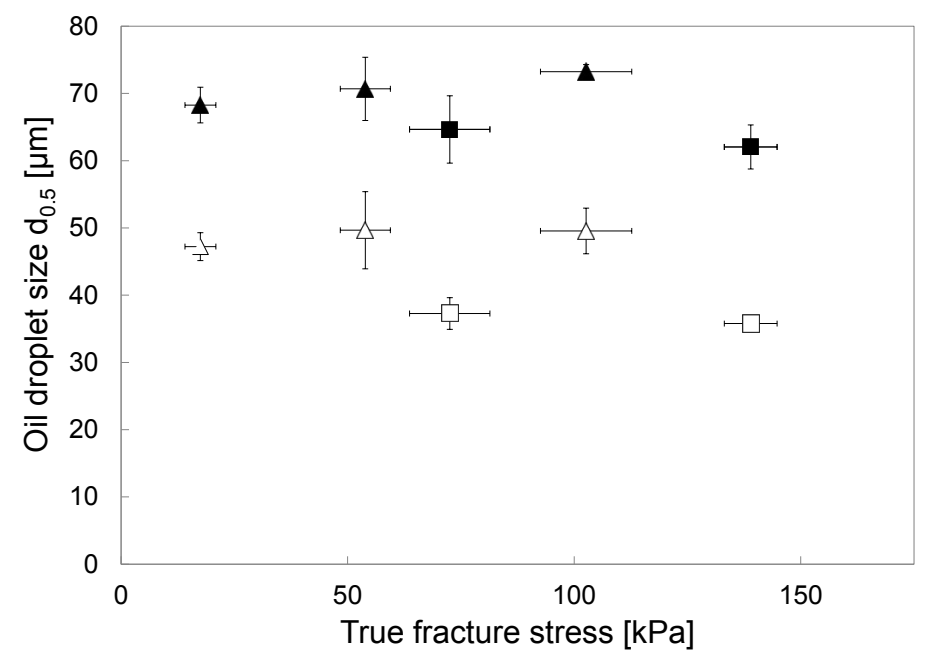

Figure 2.8 Oil droplet size plotted versus fracture stress of double $\left(w_{1} / 0 / w_{2}\right)$ emulsions with $0.4 \mathrm{wt} \% \mathrm{NaCl}$ in $\mathrm{w}_{1}$ containing either WPI (squares) or gelatin (triangles), after preparation (filled symbols) and after shear treatment (open symbols). Error bars indicate the standard deviation of the mean. 
by the fracture stress of the inner dispersed droplets, but also by the type of gelling agent used. We observed that oil droplet sizes of emulsions with different gelling agents decreased to different extents after shear treatment, resulting in a difference in the change of the yield (Figure 2.7). This indicates that the relation between oil droplet size and yield does not only depend on the mechanical properties of the gelled droplets, but also depends on the type of gelling agent. For the same difference in oil droplet sizes (before and after shear), we did not observe similar differences in yield (before and after shear) when comparing gelatin and WPI. The oil droplet breakup process may therefore be different for the gelling agents and more WPI gelled droplets are expelled during the shear treatment.

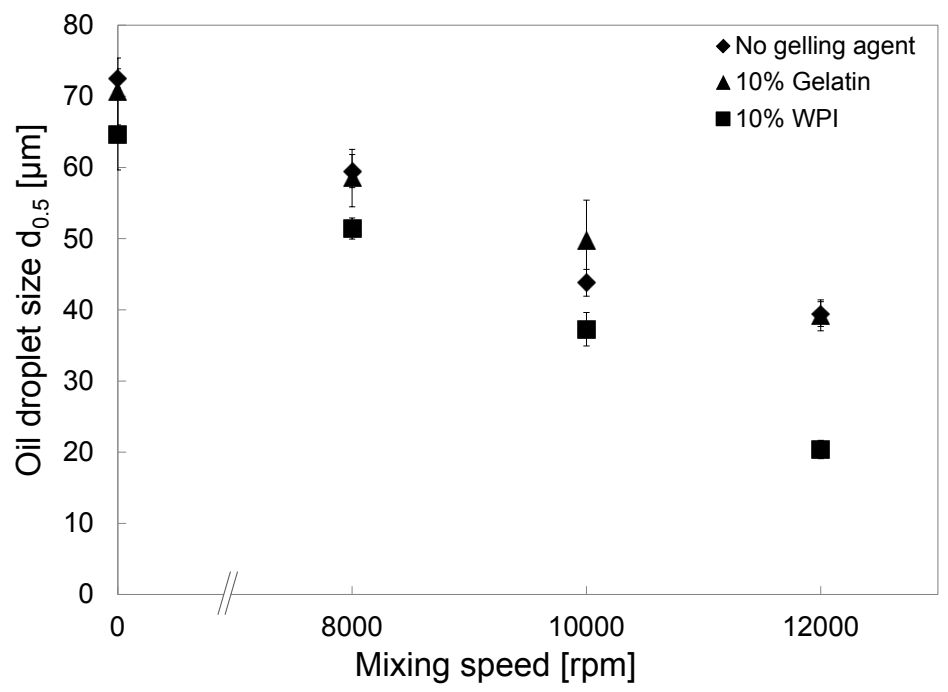

Figure 2.9 Oil droplet breakup of double $\left(w_{1} / 0 / w_{2}\right)$ emulsions with $0.4 \mathrm{wt} \% \mathrm{NaCl}$ in $\mathrm{w}_{1}$ as a function of mixing speed (Ultra Turrax). Diamonds refer to emulsions without gelling agent, triangles refer to emulsions containing $10 \mathrm{wt} \%$ gelatin, and squares refer to emulsions containing $10 \mathrm{wt} \% \mathrm{WPI}$. Error bars indicate the standard deviation of the mean.

We therefore investigated the oil droplet break up by exposing the double emulsions to different shear conditions (given as the rpm in the high speed blender). The differences in oil droplet break up are shown in Figure $\mathbf{2 . 9}$ for reference emulsions without gelling agents (diamonds), gelled with WPI (squares) and gelatin (triangles). The initial oil droplet sizes, indicated at $0 \mathrm{rpm}$, were between 65 and $72 \mu \mathrm{m}$. For all emulsions, oil droplet sizes decreased with increasing rpm. However, at $12.000 \mathrm{rpm}$, we see a large difference between the different systems: when WPI was used, oil droplets decreased in size to $20.4 \mu \mathrm{m}( \pm 1.3)$, while for gelatin, oil droplet decreased in size to $39 \mu \mathrm{m}( \pm 2.2)$. Thus, the oil droplets remain much larger than in the case of double emulsions with WPI. This shows that also the droplet size depends on the gelling agent, and may therefore influence the yield. 
The results indicate that there is a relation between yield, fracture stress and oil droplets size, which depends on the type of gelling agent used. To visualize this relation, we have plotted the yield versus the oil droplet size (altered by changing the shear conditions), for the different gelling agents at 10 wt\% (Figure 2.10). Emulsion properties depend on the type of gelling agent. Double emulsions without a gelled inner dispersed phase (diamonds) increased in yield with increasing oil droplet size. The same trend was observed for emulsions with WPI (squares) but for comparable oil droplet sizes, the yield was roughly $30 \%$ higher. The yield of double emulsions with gelatin, however, increased to a smaller extent with increasing oil droplet size, and showed an almost constant yield as a function of oil droplet size. As already discussed before, we did not see large differences in yields for both gelled emulsions compared to the non-gelled emulsions at similar oil droplet sizes.

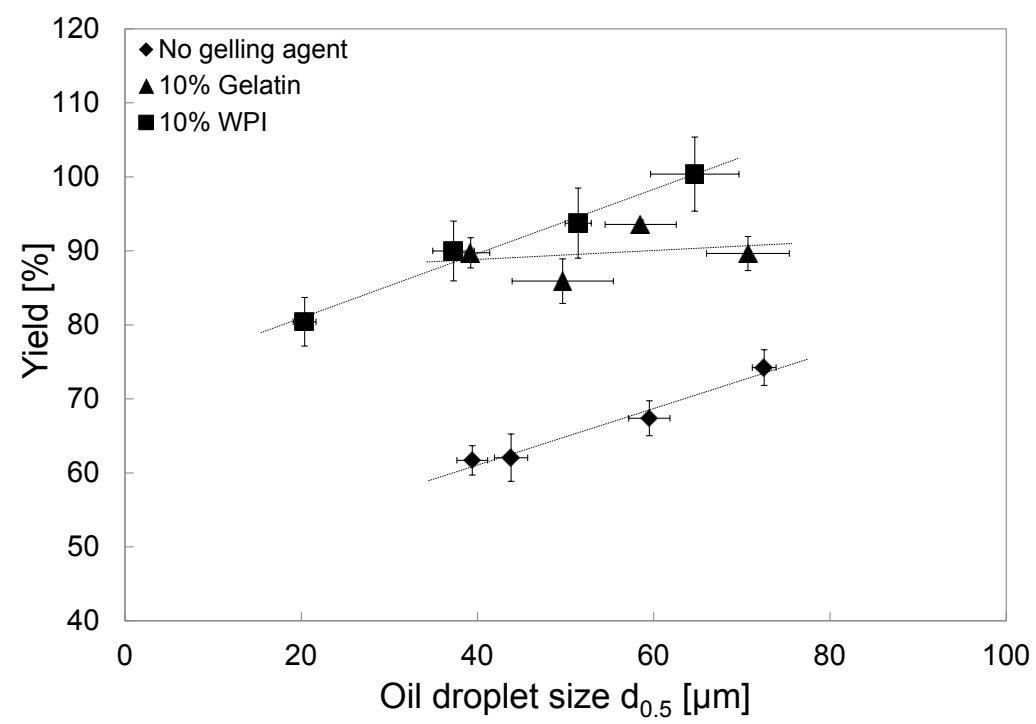

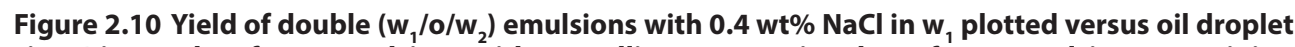
size. Diamonds refer to emulsions without gelling agent, triangles refer to emulsions containing $10 \mathrm{wt} \%$ gelatin, and squares refer to emulsions containing $10 \mathrm{wt} \%$ WPI. Error bars indicate the standard deviation of the mean. The dotted lines are added to guide the eye.

We conclude that yield and oil droplet size of double emulsions, as well as mechanical properties of gelled inner droplets, are related to each other. The relationship between these parameters depends on the type of gelling agent used. However, the exact relationship between the individual parameters is not known, as it is very difficult to control these parameters separately to correlate direct relationships between them. To gain a better insight on correlations between all involved parameters, a well-designed study may be required. 


\subsection{Conclusions}

Our aim was to investigate the effect of osmotic pressure tailoring and protein gelation of the inner dispersed phase on the stability and yield of double $\left(w_{1} / o / w_{2}\right)$ emulsions. By osmotic pressure tailoring, double emulsions could easily be prepared that were reasonably stable against heat and shear treatments. By gelling the inner dispersed phase the initial yield significantly increased, as well as the storage, heat and shear stability. The yield of the $w_{1}$-phase depended furthermore on both the concentration of gelling agent used, and the oil droplet size. Double emulsions with gelling agents were less sensitive towards an imbalance in osmotic pressure compared to their counterparts without gelling agents. The mechanical properties of a gelled inner dispersed phase seemed to influence the yield and the stability of double emulsions. Yield increased with increasing fracture stress and modulus, and differed between the gelling agents used. To gain a deeper understanding of correlations between all involved parameters (mechanical properties of inner dispersed droplets, type of gelling agent used, droplet size, effect on initial yield and shear stability), a more systematic study may be required that varies these parameters separately.

\section{Acknowledgements}

The research leading to these results has received funding from the European Union Seventh Framework Programme (FP7/2007-2013) under grant agreement nr. 289397 (TeRiFiQ project). We would like to acknowledge Herman de Beukelaer for his help with the DSC measurements. We would like to thank Prof. Schuchmann for her hospitality during a short research stay of AKLO and MR at the Department of Food Process Engineering at the Karlsruhe Institute of Technology in Karlsruhe, Germany. We would also like to thank Natalia Perez-Moral and Peter Wilde for hosting AKLO during a short research stay at the Institute of Food Research in Norwich, UK. 


\section{References}

1. Muschiolik, G., Multiple emulsions for food use. Current Opinion in Colloid \& Interface Science, 2007. 12: p. 213-220.

2. Tang, S.Y., M. Sivakumar, and B. Nashiru, Impact of osmotic pressure and gelling in the generation of highly stable single core water-in-oil-in-water (W/O/W) nano multiple emulsions of aspirin assisted by two-stage ultrasonic cavitational emulsification. Colloids Surfaces, B, 2013. 102: p. 653-658.

3. Surh, J., et al., Preparation and characterization of water/oil and water/oil/water emulsions containing biopolymer-gelled water droplets. Journal of Agricultural and Food Chemistry, 2007. 55: p. 175-184.

4. Su, J., J. Flanagan, and H. Singh, Improving encapsulation efficiency and stability of water-inoil-in-water emulsions using a modified gum arabic (Acacia (sen) SUPER GUM ${ }^{\mathrm{TM}}$ ). Food Hydrocolloids, 2008. 22: p. 112-120.

5. Su, J., et al., Synergistic effects of polyglycerol ester of polyricinoleic acid and sodium caseinate on the stabilisation of water-oil-water emulsions. Food Hydrocolloids, 2006. 20: p. 261-268.

6. Benichou, A., A. Aserin, and N. Garti, W/O/W double emulsions stabilized with WPI-polysaccharide complexes. Colloids and Surfaces A: Physicochemical and Engineering Aspects, 2007. 294: p. 20-32.

7. Lutz, R., et al., Double emulsions stabilized by a charged complex of modified pectin and whey protein isolate. Colloids and Surfaces B: Biointerfaces, 2009. 72: p. 121-127.

8. Márquez, A.L., G.G. Palazolo, and J.R. Wagner, Water in oil (w/o) and double (w/o/w) emulsions prepared with spans: Microstructure, stability, and rheology. Colloid and Polymer Science, 2007. 285: p. 1119-1128.

9. Sapei, L., M.A. Naqvi, and D. Rousseau, Stability and release properties of double emulsions for food applications. Food Hydrocolloids, 2012. 27: p. 316-323.

10. Schuch, A., et al., Influence of the second emulsification step during production of W/O/W multiple emulsions: Comparison of different methods to determine encapsulation efficiency in W/O/W emulsions. The Canadian Journal of Chemical Engineering, 2014. 92: p. 203-209.

11. Schuch, A., K. Köhler, and H.P. Schuchmann, Differential scanning calorimetry (DSC) in multiple W/O/W emulsions. Journal of Thermal Analysis and Calorimetry, 2013. 111: p. 1881-1890.

12. Florence, A.T. and D. Whitehill, Some features of breakdown in water-in-oil-in-water multiple emulsions. Journal of Colloids and Interface Science, 1980. 79: p. 243-256.

13. Mezzenga, R., B.M. Folmer, and E. Hughes, Design of double emulsions by osmotic pressure tailoring. Langmuir, 2004. 20: p. 3574-3582.

14. Jiao, J. and D.J. Burgess, Multiple Emulsion Stability: Pressure Balance and Interfacial Film Strength, in Multiple Emulsions: Technology and Applications, A. Aserin, Editor. 2008, John Wiley \& Sons, Inc.: Hoboken, NJ. p. 1-27.

15. Pawlik, A., P.W. Cox, and I.T. Norton, Food grade duplex emulsions designed and stabilised with different osmotic pressures. Journal of Colloid and Interface Science, 2010. 352: p. 59-67.

16. Iqbal, S., et al., Controlling W/O/W multiple emulsion microstructure by osmotic swelling and internal protein gelation. Food Research International, 2013. 54: p. 1613-1620.

17. Schuch, A., J. Wrenger, and H.P. Schuchmann, Production of W/O/W double emulsions. Part II: Influence of emulsification device on release of water by coalescence. Colloids and Surfaces A: Physicochemical and Engineering Aspects, 2013. 461: p. 344-351

18. Dickinson, E., Double Emulsions Stabilized by Food Biopolymers. Food Biophysics, 2011. 6: p. 1-11.

19. McClements, D.J., Food emulsions: principles, practices, and techniques. CRC series in contemporary food science. 2005, Boca Raton, FL: CRC Press.

20. Surh, J., Influence of Xanthan, Emulsification Temperature, and Environmental Stresses on the Preparation of Water-in-Corn Oil Emulsions Droplets Coated by Polyglycerol Polyricinoleate. Food Science and Biotechnology, 2009. 18: p. 299-306.

21. McClements, D.J., Protein-stabilized emulsions. Current Opinion in Colloid \& Interface Science, 2004. 9: p. 305-313.

22. Perez-Moral, N., S. Watt, and P. Wilde, Comparative study of the stability of multiple emulsions containing a gelled or aqueous internal phase. Food Hydrocolloids, 2014. 42: p. 215-222

23. Florence, A.T. and D. Whitehill, Stabilization of water/oil/water multiple emulsions by polymerization of the aqueous phases. Journal of Pharmacy and Pharmacology, 1982. 34: p. 687-691. 
24. Hemar, Y., et al., Encapsulation of Resveratrol Using Water-in-Oil-in-Water Double Emulsions. Food Biophysics, 2010. 5: p. 120-127.

25. Fechner, A., et al., Stability and release properties of double-emulsions stabilised by caseinatedextran conjugates. Food Hydrocolloids, 2007. 21: p. 943-952.

26. O'Regan, J. and D.M. Mulvihill, Sodium caseinate-maltodextrin conjugate stabilized double emulsions: Encapsulation and stability. Food Research International, 2010. 43: p. 224-231.

27. Mun, S., et al., Influence of environmental stresses on the stability of W/O/W emulsions containing enzymatically modified starch. Carbohydrate Polymers, 2013. 92: p. 1503-1511.

28. Chanamai, R. and D.J. McClements, Comparison of gum arabic, modified starch, and whey protein isolate as emulsifiers: Influence of $\mathrm{pH}_{,} \mathrm{CaCl}_{2}$ and temperature. Journal of Food Science, 2002. 67: p. 120-125.

29. Walstra, P., Physical Chemistry of Foods. Food Science and Technology, ed. O.R. Fennema, et al. Vol. 121. 2003, New York: Marcel Dekker, Inc.

30. Muschiolik, G., et al. Multiple Emulsions - Preparation and Stability. in IUFoST World Congress 13th World Congress of Food Science \& Technology. 2006. Nantes, France.

31. Law, T.K., T.L. Whateley, and A.T. Florence, Stabilisation of $w-o-w$ multiple emulsions by interfacial complexation of macromolecules and nonionic surfactants. Journal of Controlled Release, 1986. 3: p. 279-290.

32. Pays, K., et al., Double emulsions: How does release occur? Journal of Controlled Release, 2002. 79(1-3): p. 193-205.

33. Saglam, D., et al., Relation between gelation conditions and the physical properties of whey protein particles. Langmuir, 2012. 28(16): p. 6551-6560. 


\section{Chapter 3}

\section{Effect of outer water phase composition on oil droplet size and yield of double $\left(w_{1} / 0 / w_{2}\right)$ emulsions}
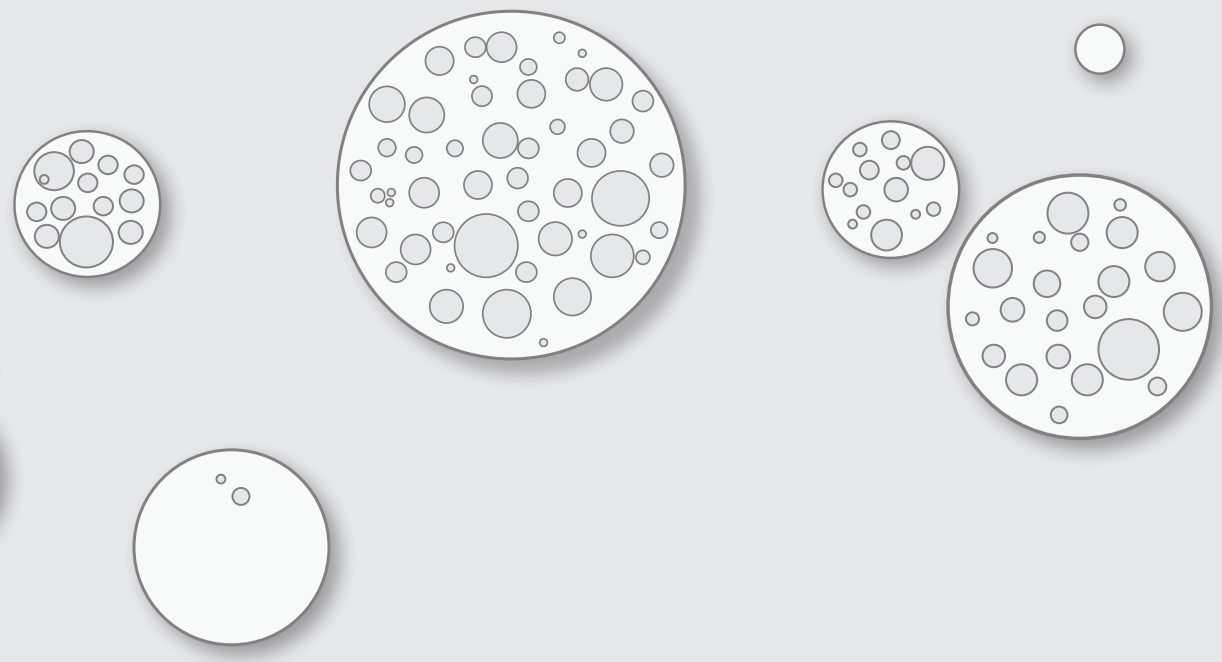

This chapter has been submitted as

AKL Oppermann, JME Noppers, M Stieger, E Scholten, Effect of outer water phase composition on oil droplet size and yield of double $\left(w_{1} / o / w_{2}\right)$ emulsions 


\begin{abstract}
In this study, the effect of various emulsifiers (whey protein isolate (WPI), Na-caseinate, and Tween 20) and thickeners (xanthan and pectin) present in the outer water phase, $\mathrm{w}_{2^{\prime}}$ on oil droplet size and yield of the inner water phase, $\mathrm{w}_{1}$, of double emulsions was investigated.
\end{abstract}

Double emulsions without thickeners stabilized by Tween 20 had smaller oil droplet sizes and higher yields in comparison to double emulsions stabilized by WPI and $\mathrm{Na}$-caseinate. This is likely due to faster diffusion of Tween 20 towards the $\left(\mathrm{o} / \mathrm{w}_{2}\right)$ interface. Gelation of the inner water droplets $\mathrm{w}_{1}$ increased yield by $20 \%$ for all emulsifiers. Gelation of $w_{1}$ droplets limits the expulsion/coalescence with the $w_{2}$ phase. Upon the addition of thickeners, the increasing viscosity of the outer water phase $w_{2}$ decreased the viscosity ratio $\eta_{w 1 / 0}: \eta_{w 2}$ and thereby facilitated oil droplet breakup. This resulted in smaller oil droplets and lower yields for all hydrophilic emulsifiers. When pectin was used as a thickener, in comparison to xanthan, an additional decrease in yield was observed indicating possible competition of pectin with hydrophilic emulsifiers at the $\left(\mathrm{o} / \mathrm{w}_{2}\right)$ interface. In this case, the yield decreased to values close to zero indicating that all inner water droplets $w_{1}$ were lost during emulsification.

We conclude that type of hydrophilic emulsifier, mechanical properties of inner water droplets, viscosity ratio of continuous and dispersed phase, as well as type of thickener influence oil droplet size and yield of $w_{1}$ phase of double emulsions. This work contributes to a better understanding of the physical-chemical properties of double emulsions in relation to composition, which could potentially be used to design double emulsions as fat replacers in more complex food systems. 


\subsection{Introduction}

Double emulsions such as water-in-oil-in-water $\left(\mathrm{w}_{1} / \mathrm{o} / \mathrm{w}_{2}\right)$ emulsions are complex multiphase systems that consist of a water-in-oil $\left(w_{1} / 0\right)$ emulsion dispersed in an outer water phase $\mathrm{w}_{2}$. Double emulsions can potentially be used to reduce oil content in foods [1]. However, double emulsions are very prone to destabilization. For example, inner water droplets $w_{1}$ may be lost irreversibly to the outer water phase $w_{2}$ by diffusion processes or coalescence of $w_{1}$ droplets with the outer water phase $w_{2}[2,3]$. Both mechanisms are influenced by the characteristics of the $\left(0 / w_{2}\right)$ interfaces and by the size of the oil droplets. The oil droplet size in turn is determined by preparation process parameters such as shear stresses, and therefore depends on the characteristics of the continuous phase $[4,5]$.

To stabilize double emulsions, diffusion and coalescence should be minimized. The choice of a suitable emulsifier is crucial for double emulsion stability [6]. Since there are two interfaces, it is necessary to have two emulsifiers, a lipophilic one to stabilize the $w_{1} / o$ interface, and a hydrophilic one to stabilize the $o / w_{2}$ interface. The emulsifiers can interact at the interfaces, which can cause instabilities in double emulsions [7]. Many research groups have already investigated the influence of type and concentration of emulsifiers on double emulsion stability [8-11]. Chavez and co-workers showed that the type and relative amount of hydrophilic and lipophilic emulsifiers has a large influence on the probability of $w_{1}-w_{1}$ and $w_{1}-w_{2}$ coalescence, and therefore on double emulsion stability [10].

To retain the inner water droplets within the oil droplets, oil droplets in double emulsions are larger than in single emulsions. Therefore, they are more prone to creaming. Next to changes at the interface by adsorption of emulsifiers, the stability of food emulsions can also be accomplished by changes in the bulk phase. Thickeners can be used to increase the viscosity of the continuous water phase $\mathrm{w}_{2}$. The increase in viscosity reduces the mobility of oil droplets and therefore prevents creaming and coalescence. Simultaneously, the viscosity of the continuous water phase influences the oil droplet size, since droplet breakup is determined by the shear stresses exerted at the droplet surface [8]. Both an increase in viscosity and decrease in droplet size will limit creaming of oil droplets and increase stability.

While physical stability (reduction of creaming) can be enhanced by the addition of thickeners, the changes in the bulk phase may also influence the coalescence of the inner water droplets. Several studies investigated the influence of surfactant and polysaccharide type on the release of inner water droplets during preparation of 
double emulsions $[4,6,9,12,13]$. Lutz and co-workers [14] studied the effect of pectin addition on stability of whey protein isolate-stabilized double emulsions at different $\mathrm{pH}$ values. They found that the stability of the emulsions increased, which was attributed to protein-pectin complex formation at the interface, thereby improving the emulsion stability through steric stabilization.

Although some studies have been performed, a complete understanding on the relations between the interfacial and bulk characteristics of double emulsions and oil droplet size and yield is still lacking. To eventually apply double emulsions as fat replacers in foods, it is essential that the $w_{1}$ water is kept inside the oil droplets during preparation and storage. Only double emulsions with a high inner water phase content (high yield) will be effective as a fat replacer. It is desired to gain further insights in the effect of emulsion characteristics on the yield of double emulsions. The aim of the present study is to investigate the influence of various hydrophilic emulsifiers and thickeners present in the outer water phase $\left(w_{2}\right)$ on oil droplet size and yield of inner water phase $\left(w_{1}\right)$ of double emulsions. In particular, we aim to understand the influence of viscosity of the outer water phase by using xanthan and high-methoxyl (HM) pectin on double emulsions stabilized by hydrophilic low molecular weight surfactants (Tween 20) and proteins (Na-caseinate and whey protein isolate), containing a gelled or non-gelled inner water phase $w_{1}$. Tween 20 was chosen as a small molecular weight surfactant, Na-caseinate as a flexible protein, and whey protein isolate (WPI) as a globular protein.

\subsection{Materials and methods}

\subsubsection{Materials}

Hydrophilic emulsifiers used in this study were Tween 20 (Polysorbate 20, Lot number SZBE1620V, Sigma-Aldrich, Seelze, Germany), Na-caseinate (EM 7, Lot number 10676648, FrieslandCampina, Veghel), and whey protein isolate (WPI, BiPro JE-099-2-420) obtained from Davisco Foods International Inc. (Le Sueur, MN). The composition of WPI as stated by the manufacturer was $97.7 \%$ protein, $0.3 \%$ fat, $1.8 \%$ ash (dry weight basis). Xanthan gum (Keltrol ${ }^{\circledR}$ Advanced Performance) and pectin (Pectin Classic CU 201) were used as thickeners. Xanthan gum was kindly provided by CP Kelco (Levallois-Perret, France), while pectin was kindly provided by Herbstreith \& Fox KG (Neuenbürg, Germany). Pectin had a degree of esterification (DE) of $69 \%$, and a galacturonic acid content of $82 \%$. Gelatin (type A, Type 250 PS 30) from Rousselot (Gent, Belgium) was used. For all aqueous solutions, purified water (Milli-Q, 
18.2 $\mathrm{M} \Omega \mathrm{cm}$ at $25^{\circ} \mathrm{C}$ ) was used. $\mathrm{NaCl}$ was obtained from Sigma-Aldrich (purity $\geq 99.5 \%$, Sigma-Aldrich, Steinheim, Germany). Commercial sunflower oil was purchased from a local retailer (Wageningen, the Netherlands). Polyglycerol polyricinoleate (PGPR 4175) was kindly provided by Palsgaard (Juelsminde, Denmark).

\subsubsection{Preparation of double $\left(w_{1} / 0 / w_{2}\right)$ emulsions}

\subsubsection{Preparation of solutions}

For the inner aqueous phase $\mathrm{w}_{1}, \mathrm{NaCl}$ solutions were prepared by dissolving $\mathrm{NaCl}$ in purified water while stirring. Gelatin solutions were prepared by dissolving $10 \mathrm{wt} \%$ gelatin and $\mathrm{NaCl}$ in purified water at $60{ }^{\circ} \mathrm{C}$ while stirring. The solutions were kept at $60^{\circ} \mathrm{C}$ until the gelatin was completely dissolved. Gelatin was used to gel the inner water droplets in some of the emulsions. The concentration of $\mathrm{NaCl}$ in the inner aqueous phase $w_{1}$ was adapted based on the osmotic pressure of the outer aqueous phase $w_{2}$ and differed between $0.4 \mathrm{wt} \%$ and $2.2 \mathrm{wt} \%$. The amount of $\mathrm{NaCl}$ was chosen in such a way that no water transport between the inner and outer water phase was observed. The oil phase was prepared by adding 4 wt\% PGPR, as the lipophilic emulsifier, to sunflower oil followed by 30 min of stirring at $20^{\circ} \mathrm{C}$. The outer aqueous phase $\mathrm{w}_{2}$ was prepared by adding $0.2 \mathrm{wt} \% \mathrm{NaCl}$ and either $1 \mathrm{wt} \% \mathrm{WPI}, 1 \mathrm{wt} \%$ Tween 20 or $1 \mathrm{wt} \%$ Na-caseinate, as the hydrophilic emulsifier, to purified water while stirring. Stirring was continued for $2 \mathrm{~h}$ at $20{ }^{\circ} \mathrm{C}$ to ensure complete dissolution of the emulsifiers. For outer water phases containing thickeners, solutions were heated to $55^{\circ} \mathrm{C}$ after the emulsifier was dissolved. For xanthan, the solutions were prepared by adding xanthan at concentrations between $0.0-0.5 \mathrm{wt} \%$ while stirring until dissolved. Outer water phases containing pectin were prepared by adding $4 \mathrm{wt} \%$ pectin to the heated emulsifier solutions while stirring until dissolved. This stock solution was then diluted with the outer water phase containing no thickener to obtain the required pectin concentrations $(0.5-4 \mathrm{wt} \%)$. The concentrations of pectin were about ten times higher than for xanthan to obtain a similar viscosity range of the outer water phase. The final $\mathrm{pH}$ of both the xanthan and pectin solution was approximately 6.8 . When necessary, the $\mathrm{pH}$ of the pectin solutions was re-adjusted with hydrochloric acid or sodium hydroxide to $\mathrm{pH} 6.8 \pm 0.1$. All solutions were stored at $4{ }^{\circ} \mathrm{C}$ overnight before use.

\subsubsection{Preparation of $\left(w_{1} / 0\right)$ emulsions}

Primary water-in-oil $\left(w_{1} / o\right)$ emulsions were prepared by adding $30 \mathrm{wt} \%$ of aqueous phase $\mathrm{w}_{1}$ to the oil phase in a $1000 \mathrm{~mL}$ stainless steel container of a Waring blender 8011 ES (Stamford, CT), and mixing at 22000 rpm for 60 s. For samples with gelatin in 
the inner aqueous phase, both the oil phase and the gelatin solution were heated to $60{ }^{\circ} \mathrm{C}$ in a water bath prior to mixing. Samples were subsequently cooled under tap water for at least $15 \mathrm{~min}$.

\subsubsection{Preparation of double $\left(w_{1} / 0 / w_{2}\right)$ emulsions}

Double emulsions were prepared as described previously in chapter 2 . Thirty $w t \%$ of the primary $\left(w_{1} / 0\right)$ emulsion was dispersed into $70 w t \%$ of outer aqueous phase $\left(w_{2}\right)$ while increasing the mixing speed of the high speed blender (Ultra Turrax T25 with the dispersing tool S25-N 18G, IKA, Staufen, Germany) from 3000 rpm to 7000 rpm within $1 \mathrm{~min}$. Mixing was continued for $4 \mathrm{~min}$ at $7000 \mathrm{rpm}$. All emulsions were prepared in duplicate. Oil droplet size and yield were analyzed on the day of emulsion preparation.

\subsubsection{Emulsion characterization}

\subsubsection{Viscosity of outer water phase $\mathbf{w}_{2}$}

Viscosity measurements of the outer water phase $w_{2}$ and $\left(w_{1} / 0\right)$ emulsions were performed with a Modular Compact Rheometer 302 (MCR 302, Anton Paar, Grax, Austria). The geometry used was a double gap cylinder (C-DG 26.7/Ti SN 3526, gap width $0.40 \mathrm{~mm}$ ). Measurements were performed at $20^{\circ} \mathrm{C}$ with a sample volume of $3.8 \mathrm{~mL}$. The viscosity was recorded for an increasing shear rate from $0.01 \mathrm{~s}^{-1}$ to $1000 \mathrm{~s}^{-1}$ within $16 \mathrm{~min}$. Each outer water phase was measured in duplicate to obtain an averaged value. For calculations of viscosity ratios $\lambda$, the viscosities at shear rates $\dot{\gamma}$ of $100 \mathrm{~s}^{-1}$ and $1000 \mathrm{~s}^{-1}$ were used $\left(\lambda_{100 \mathrm{~s}-1}\right.$ and $\left.\lambda_{1000 \mathrm{~s}-1}\right)$. The viscosity ratio $\lambda$ was obtained by dividing the viscosity of the dispersed $\left(w_{1} / 0\right)$ phase $\left(\eta_{d}\right)$ by the viscosity of the continuous phase $w_{2}\left(\eta_{c}\right)$ as $\lambda=\eta_{d} / \eta_{c}$.

\subsubsection{Osmotic pressure measurements}

The osmolality of aqueous solutions of the outer water phase was measured in triplicate using a freezing point depression Osmometer (Advanced Instruments, Model 3320, Norwood, US). A sample holder was loaded with $20 \mu \mathrm{L}$ of sample and inserted in the sample port of the instrument. Results are expressed in osmolality ( $\mathrm{mOsmol} / \mathrm{kg})$.

\subsubsection{Oil droplet size measurements}

Oil droplet sizes of all double $\left(\mathrm{w}_{1} / \mathrm{o} / \mathrm{w}_{2}\right)$ emulsions were measured by static light scattering with a Mastersizer 2000 (Malvern Instruments, Worcestershire, UK). The refractive index of the oil droplets was set at 1.45 and the one of demineralized water (continuous phase) at 1.33. Absorption was set at 0.01 . The size distribution of each sample was measured three times. Average sizes are reported as $\mathrm{d}_{0.5}$. 


\subsubsection{Yield measurements}

DSC measurements were carried out to determine the yield of double emulsions. Principles of the method has been previously described by Schuch and co-workers [15]. Yield is defined as the percentage of inner aqueous phase entrapped in the oil phase after emulsion preparation, compared to the volume fraction of inner water droplets of the initial primary emulsion. A sample mass between 7 and $12 \mathrm{mg}$ was filled in an aluminum pan, hermetically sealed and introduced into the calorimeter (Diamond DSC (Perkin Elmer, Pyris, USA)). After equilibrating at $10^{\circ} \mathrm{C}$ for $1 \mathrm{~min}$, the samples were steadily cooled to $-60{ }^{\circ} \mathrm{C}$ at a cooling rate of $5{ }^{\circ} \mathrm{C}$ per minute. Details of the method and data analysis have been described in chapter 2.

\subsection{Results and discussion}

\subsubsection{Effect of hydrophilic emulsifiers on oil droplet size and yield of $w_{1}$ phase}

First, we investigated the effect of hydrophilicemulsifier type on oil droplet size and yield of inner $w_{1}$ phase of double emulsions without thickener in the outer water phase $w_{2}$. We have chosen Tween 20 as a small molecular weight surfactant, Na-caseinate as a flexible protein, and whey protein isolate (WPI) as a globular protein. Figure 3.1 shows the yield of double emulsions stabilized by Tween 20, WPI or Na-caseinate, as a function of oil droplet size (filled symbols). As it is known that gelation of the inner water droplets has a large influence on the yield of the double emulsion (chapter 2), we also included a series of double emulsions in which the inner water droplets were gelled (open symbols). In the case of non-gelled $w_{1}$ droplets, double emulsions stabilized with Tween 20 had smaller oil droplets $(51 \mu \mathrm{m})$ than those stabilized with proteins ( 62 and $72 \mu \mathrm{m}$ ). This difference may be explained by considering typical emulsifier characteristics such as structural composition, molecular weight, rate of interfacial adsorption and ability to decrease interfacial tension. Low molecular weight surfactants like Tween 20 diffuse faster to an oil-water interface and lower the interfacial tension to a greater extent than high molecular weight surfactants such as proteins [16]. This explains why Tween 20-stabilized emulsions had smaller oil droplets. Proteins, in contrast, diffuse relatively slow to the interface [17], which explains the larger oil droplet sizes for both the WPI- and Na-caseinate-stabilized double emulsions. However, also between the two proteins, differences in oil droplet sizes were observed. Na-caseinate-stabilized emulsions yielded smaller oil droplets than WPI-stabilized emulsions. Also Cofrades and co-workers [18] found smaller oil droplet sizes of double emulsions when Na-caseinate was used, in comparison 
to whey protein-stabilized double emulsions. Both WPI and Na-caseinate lower the interfacial tension of an oil-water interface to similar extents $[17,19,20]$, so based on the interfacial tensions, one would expect similar oil droplet sizes. The difference in oil droplet size probably comes from a difference in the diffusion rate or the structural characteristics of the proteins. Caseinates are more flexible and have more exposed hydrophobic groups than globular WPI proteins. Due to the large flexibility, caseinate can rearrange at the interface, which contributes to their rapid adsorption to oil droplet surfaces during emulsification [17, 21-23]. The flexible nature of caseinate allows for more effective surface coverage of the interfacial area, in comparison to WPI [23]. The faster diffusion and more effective surface coverage might explain the smaller oil droplet sizes for Na-caseinate-stabilized double emulsions compared to WPI-stabilized double emulsions.

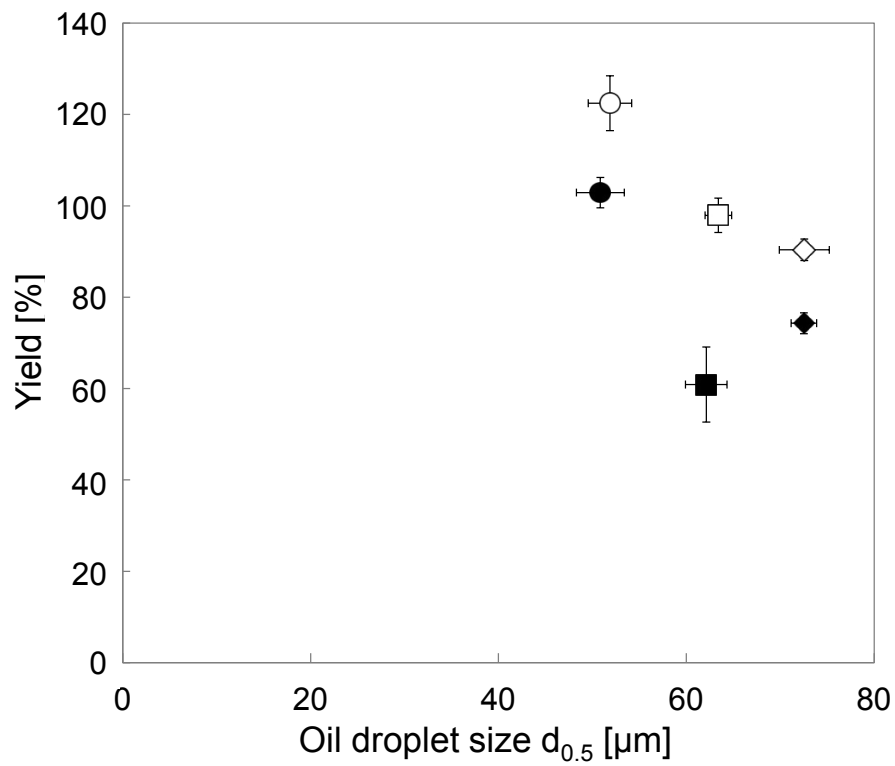

Figure 3.1 Yield of double emulsions versus oil droplet size for different hydrophilic emulsifiers. Filled symbols refer to non-gelled inner aqueous phases $w_{1}$ and open symbols to gelled inner aqueous phases $w_{1}$. Double emulsions were stabilized by WPI (diamonds), Tween 20 (circles), or $\mathrm{Na}$-caseinate (squares). Error bars indicate standard deviations.

In addition to differences in oil droplet size, differences in yield of the inner $w_{1}$ phase were observed. Tween 20-stabilized double emulsions resulted in the highest yields (about $100 \%$ ), whereas protein-stabilized double emulsions had lower yields (between 60 and $75 \%$ ), even though the same preparation procedure was applied. Similar yields have been found before for double emulsions stabilized by Tween 20 $[9,24]$ and WPI ([20] and chapter 2). However, it is difficult to compare those 
results, since the emulsion composition and oil droplet sizes usually differ, as well as the method used to determine yield. We hypothesize that the increased yield of Tween 20-stabilized emulsions is related to the fast adsorption kinetics of the low molecular weight surfactant during emulsion breakup. A fast adsorption onto the interface increases the interfacial thickness (and therefore interfacial viscosity), which limits the expulsion of the inner water droplets to the outer water phase through the interfacial layer. A fast adsorption of Tween 20 at the newly-created oil-water interface might therefore not only explain the smaller oil droplet sizes, but also the higher yields in comparison to protein-stabilized double emulsions.

Regarding the effect of gelation of the inner water droplets $w_{1}$, we found no differences in oil droplet size for the different hydrophilic emulsifiers used. Oil droplet breakup is governed by two factors: interfacial characteristics and deformability of the oil droplets (determined by the viscosity ratio of the dispersed phase and the continuous phase). Gelation of the oil droplets may decrease the deformability of the oil droplets, thereby decreasing the oil droplet breakup. The fact that no difference in oil droplet size was observed indicates that gelation did not change the deformability of oil droplets to a large extent, and suggests that the interfacial properties of the oilwater interface dominated the oil droplet breakup.

Regarding the yield, an increase of at least $20 \%$ was found for the three emulsions when the inner water phase was gelled. This finding is in agreement with our previous study (chapter 2). The improved yield with gelled inner $w_{1}$ droplets is due to an increased stiffness of the water droplets, which increases the energy barrier to expel the gelled droplets to the outer water phase. In our previous study, only WPI was used as hydrophilic emulsifier. It is observed that gelation also led to a similar increase in yield when Tween 20 or Na-caseinate were used. Even when the $\left(0 / w_{2}\right)$ interface was covered fast as a result of the fast diffusion of Tween 20, gelation led to even less expulsion of the inner water phase. The rate of coalescence between the inner $w_{1}$ and the outer $w_{2}$ phase seemed to be influenced by both the gelation (and therefore deformability of inner water droplets) as well as by the $\left(\mathrm{o} / \mathrm{w}_{2}\right)$ interfacial properties. The yield was enhanced when merging of the inner water droplets with the outer water phase was inhibited due to the presence of an interfacial layer and reduced deformability of the inner water phase.

The gelation of the Tween 20-stabilized double emulsions increased the yield to $120 \%$. This indicates that more water was included inside the oil droplets than initially added during the emulsification. These results suggest that additional water was included by an excess of Tween 20 (possibly in micellar form) present in the continuous phase. 
The difference in yields between Tween 20-stabilized double emulsions with a gelled and non-gelled inner aqueous phase is in contrast to findings from Surh and co-workers [24], who found a yield of approximately $95 \%$ for both gelled and nongelled conditions. However, since their oil droplet sizes were very small (of the order of 4-10 $\mu \mathrm{m}$ ), the additional effect of gelation may have been negligible.

Overall, these results show that the oil droplet size is determined by the rate of adsorption of the emulsifier at the interface. A faster adsorption led to less expulsion of the inner water droplets, and therefore resulted in higher yields. Gelation of the inner $w_{1}$ phase reduced the expulsion of the inner water droplets $w_{1}$ to the outer water phase $w_{2}$ even further, thereby increasing the yield even more.

\subsubsection{Effect of viscosity of outer water phase on oil droplet breakup}

To investigate the effect of the viscosity of the outer water phase on oil droplet breakup and yield of the inner $w_{1}$ phase of double emulsions, we modified the outer water phase viscosity by addition of xanthan or pectin at varying concentrations. We first discuss the results for the double emulsions containing xanthan. As no electrostatic attraction is expected between the emulsifiers and the xanthan at the $\mathrm{pH}$ of the emulsions ( $\mathrm{pH} 6.8$ ), we assume that xanthan was present in the continuous phase only, and not adsorbed at the $\left(\mathrm{o} / \mathrm{w}_{2}\right)$ interface by complex formation with the emulsifiers. The addition of the thickener to the outer water phase $w_{2}$ increased the viscosity, thereby reducing the viscosity ratio $\lambda$ and increasing the level of deformation of the oil droplets during emulsion preparation. To examine the effect of the viscosity ratios $\lambda$ on oil droplet size, we plotted the resulting oil droplet size versus the viscosity ratio determined at a shear rate of $100 \mathrm{~s}^{-1}$ for all double emulsions (Figure 3.2). The viscosities of $\left(w_{1} / 0\right)$ emulsions with a gelled and non-gelled inner aqueous phase were very similar (205 mPa.s vs. $213 \mathrm{mPa} . \mathrm{s}$ ), therefore changes in the viscosity ratio were practically related to changes in the outer water phase viscosity only. Differences for gelled and non-gelled samples were therefore also negligible.

As can be seen in Figure 3.2, oil droplet sizes decreased in a linear fashion for the different types of hydrophilic emulsifier with decreasing $\lambda$. The absolute change in oil droplet size was the smallest for Tween 20-stabilized double emulsions. The droplet size reduced by $35 \mu \mathrm{m}$ from 50 to about $15 \mu \mathrm{m}$ (decrease by $70 \%$ ). For both protein-stabilized emulsions, the droplet size decreased by $42 \mu \mathrm{m}$. Oil droplet sizes of WPI-stabilized double emulsions decreased by $58 \%$ from about 72 to about $30 \mu \mathrm{m}$, while those of NaCas-stabilized double emulsions decreased by $68 \%$ from about 62 to about $20 \mu \mathrm{m}$. Below a viscosity ratio $\lambda \approx 5$, no significant changes in droplet size were observed anymore with decreasing $\lambda$. 


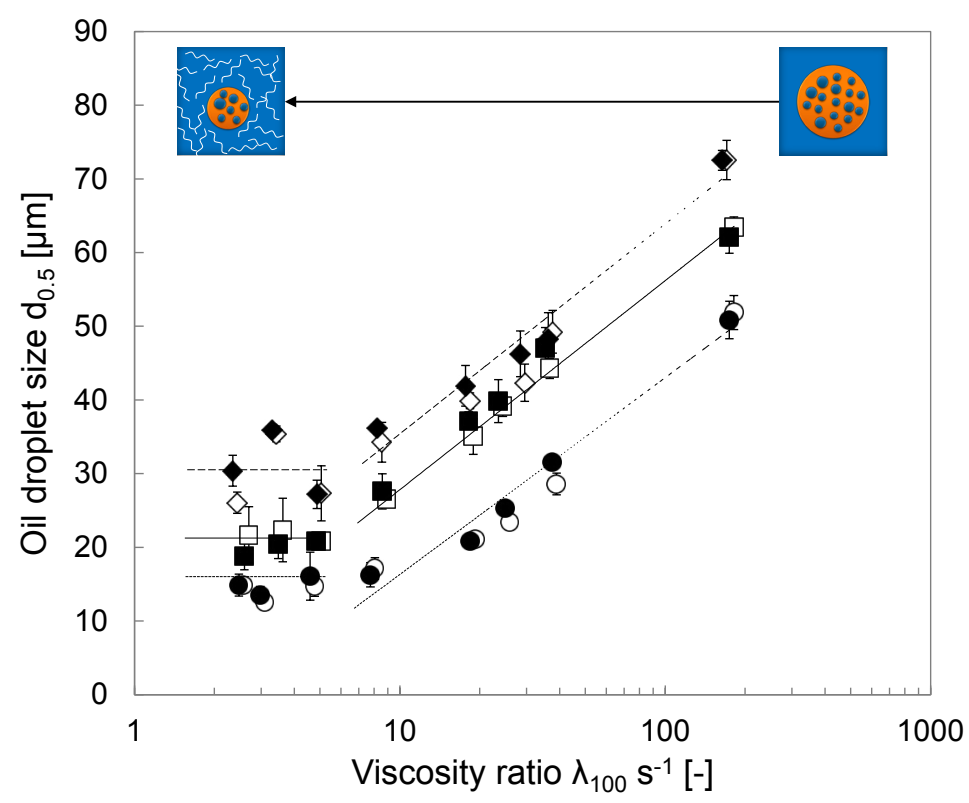

Figure 3.2 Oil droplet size of double emulsions with non-gelled (filled symbol) and gelled (open symbol) inner aqueous phase $w_{1}$ as a function of viscosity ratio $\lambda$. Viscosity of $w_{2}$ was modified by addition of xanthan. Double emulsions were stabilized by WPI (diamonds), Tween 20 (circles), or Na-caseinate (squares). Error bars indicate standard deviation. Lines (dashed for WPI, solid for $\mathrm{Na}$-caseinate and dotted for Tween 20 ) are added to guide the eye.

Even though the initial droplet sizes were different, the decrease in droplet size for an increasing outer phase viscosity (lower ratio) was similar for the three emulsifiers (visualized by the added lines). Only for low viscosity ratios, the decrease in droplet size is limited for the emulsions with Tween 20. These results indicate that the change in oil droplet size was not dependent on the initial droplet size.

Final oil droplet size is influenced by oil droplet breakup and coalescence: (1) Oil droplet coalescence decreases with increasing $w_{2}$ viscosity, and oil droplet breakup is influenced by (2) viscous forces, and (3) changes of the interfacial properties. In the following paragraph, we first discuss the influence of the viscous forces on oil droplet breakup and final droplet size.

It is well-known that emulsion droplet breakup is dependent on the deformability of the oil droplets, which is related to the forces exerted at the interface. Droplet breakup is governed by the shear stress (viscous forces) and the interfacial stress (interfacial tension), and can be expressed as the Capillary number, $\mathrm{Ca}$, which is defined as

$$
C a=\frac{\tau}{\sigma / R}=\frac{\eta_{c} \cdot \dot{\gamma} \cdot R}{\sigma}
$$


with $\sigma$ the interfacial tension, $R$ the droplet radius, $\tau$ the deforming shear stress, $\eta_{c}$ the viscosity of the continuous phase and $\dot{\gamma}$ the shear rate. The higher the viscous forces and the lower the interfacial tension, the larger the Capillary number. If the Capillary number exceeds a critical value, $\mathrm{Ca}_{\mathrm{cr}^{\prime}}$ the dominant viscous forces initiate density fluctuations in the interfaces, droplets become unstable and finally break up [25]. As the droplet breakup depends on the viscous forces, it is related to the viscosity ratio $\lambda$. For higher viscosities of the outer water phase, the viscosity ratio becomes smaller, and droplet deformation and breakup is promoted. It has been found that for single $\left(\mathrm{o} / \mathrm{w}_{2}\right.$ ) emulsions, droplet breakup is easiest when $\lambda$ is between 0.1 and 1 [25-28]. As shown in Figure 3.2, we also found a viscosity ratio, below which oil droplet breakup seemed to be easiest. In our case, this critical viscosity ratio was approximately 5 . Below this ratio, the droplet sizes were constant. This critical ratio differed from the single emulsions by one order of magnitude. Stroeve and co-workers [29] investigated droplet breakup in double emulsions in simple shear flow. They found that the dependency of the critical Capillary number on the viscosity ratio shows the same relation as for single emulsions but is shifted to higher viscosity ratios. We observe a similar shift and hypothesize that this shift is probably due to the difference in the droplet size between single and double emulsions.

Since our outer water phases contained xanthan, it was shear-thinning. Because of the shear-thinning behaviour, the actual viscosity during the emulsification might have been lower than the one used to calculate the viscosity ratios. The interpretation of the data might depend on the shear rate that is selected to estimate the viscosities used to calculate the viscosity ratios. Therefore, we compared our results based on viscosity ratios calculated from viscosities at shear rates of $100 \mathrm{~s}^{-1}$ and $1000 \mathrm{~s}^{-1}$. The results for viscosities at shear rates of $1000 \mathrm{~s}^{-1}$ (data not shown) were very similar to the results presented for shear rates of $100 \mathrm{~s}^{-1}$.

Our results of decreasing oil droplet size with decreasing viscosity ratio are in line with those found by others [29-32]. Leal-Calderon and co-workers [31] found a decrease in oil droplet size of double emulsions, when the xanthan concentration in the outer water phase was increased from 0.25 to $1 \%$. Even though they did not report the viscosities of the $w_{2}$ phase, results are comparable to ours since a similar xanthan concentration range was covered (see section 3.2.2).

Although we assume xanthan to be present mainly in the continuous phase due to lack of electrostatic attraction, some studies have discussed possible interactions between proteins and xanthan even at $\mathrm{pH}$ values where both molecules are negatively charged [33]. Such complexation between Na-caseinate and xanthan was indeed 
observed in a study by Kobori and co-workers [34]. Although weak electrostatic repulsion is present, Na-caseinate and xanthan can form weakly associated complexes through hydrophobic interactions at distinct entities. However, even though formation of complexes was observed, no changes in protein coverage of emulsions was found by Hemar and co-workers [35]. Therefore, even though some complexation may have occurred, we assume that the results were indeed dominantly due to changes in the bulk phase and that interfacial changes were negligible.

To summarize, we found a decrease in oil droplet size for lower viscosity ratios for the emulsions differing in the hydrophilic emulsifiers thickened by xanthan. The results indicated that the viscous force (shear stress) was the dominating factor influencing changes in oil droplet size and that interfacial characterisitics had a negligible influence. Below a critical viscosity ratio $\lambda$ of 5 , oil droplet sizes remained constant and did not decrease any further. As the initial droplet size (without xanthan) was different for the different types of emulsifiers, oil droplet sizes differ at the same viscosity ratio. This indicates that interfacial properties remain important when the viscous forces become more dominant, and the final oil droplet size is influenced by both the bulk and the interfacial characteristics.

\subsubsection{Effect of thickener type on oil droplet breakup}

For xanthan-thickened double emulsions, droplet breakup was mainly determined by the viscosity of the continuous phase, and no other interfacial effects seemed to play a role. To confirm that it is predominantly the viscosity ratio that influences oil droplet breakup, we used pectin as a different type of thickener. Pectin is a polysaccharide that is often used to stabilize emulsions. High-methoxyl (HM) pectin was used, and higher concentrations were used to obtain similar viscosities for xanthan-thickened double emulsions. We assume that no electrostatic attraction between pectin and the emulsifiers took place at $\mathrm{pH} 6.8$.

Figure 3.3 shows the oil droplet size as a function of the viscosity ratio. As gelation of the droplets does not lead to changes in droplet size, we only present the results of the non-gelled samples. As indicated by the trendlines, the decrease in oil droplet size (slope) was similar for all hydrophilic emulsifiers. Tween 20-stabilized double emulsions showed smaller oil droplet sizes at the same viscosity ratio in comparison to protein-stabilized emulsions. The smallest average oil droplet sizes obtained were between 7 and $9 \mu \mathrm{m}$ for all hydrophilic emulsifiers, smaller than when xanthan was used as thickener. Schmidt and co-workers [36, 37], and Lutz and co-workers [14] also found decreasing oil droplet sizes in $\left(\mathrm{o} / \mathrm{w}_{2}\right)$ emulsions upon pectin addition. 


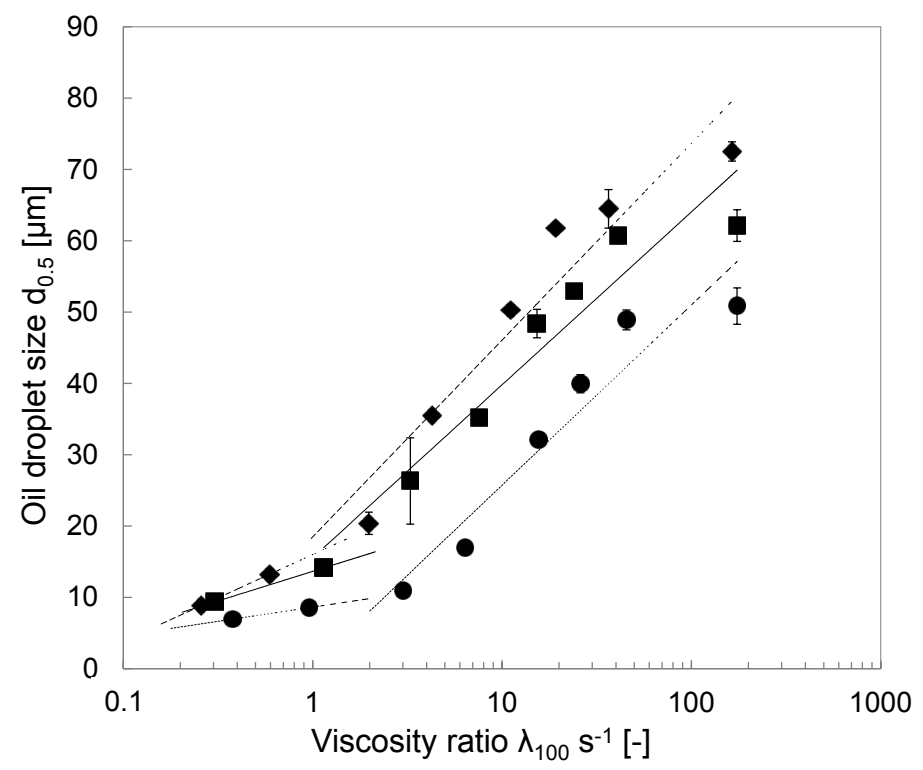

Figure 3.3 Oil droplet size of double emulsions with non-gelled inner aqueous phase as a function of viscosity ratio $\lambda$. Viscosity of $w_{2}$ was modified by addition of pectin. Double emulsions were stabilized by WPI (diamonds), Tween 20 (circles), or Na-caseinate (squares). Error bars indicate standard deviation. Lines (dashed for WPI, solid for Na-caseinate and dotted for Tween 20) are added to guide the eye.

To have a better comparison between the thickeners, we show the results for xanthan and pectin in the same figure to investigate whether oil droplet breakup is dependent on the type of thickener used. Since the changes in oil droplet size are very similar for all emulsifiers, we discuss only the results for Na-caseinate (Figure 3.4) in the following paragraphs.

When comparing double emulsions with xanthan or pectin in the outer water phase, Figure 3.4 shows that oil droplet breakup depended on the type of thickener used. For the same viscosity ratio, double emulsions with pectin showed larger oil droplet sizes than emulsions containing xanthan. Considering that xanthan is highly shearthinning, it is possible that the viscosity at a shear rate of $100 \mathrm{~s}^{-1}$ is not representative for the viscosity during emulsion preparation. It could be possible that the viscosities for xanthan are overestimated and that the viscosity is actually lower. Lower viscosities for xanthan would lead to higher values of viscosity ratio, shifting the data to the right. To take into account the shear thinning effect, we also took the viscosities at a shear rate of $1000 \mathrm{~s}^{-1}$ to calculate the viscosity ratios. However, similar results were obtained, indicating that the differences were not an effect of differences in non-Newtonian behaviour. 


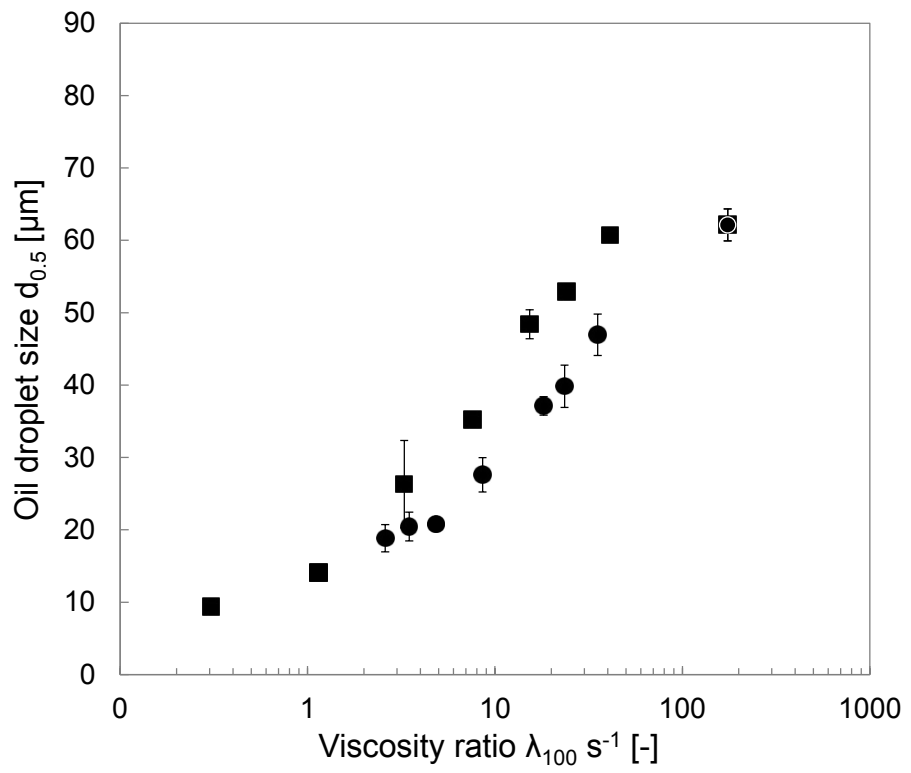

Figure 3.4 Oil droplet size as a function of viscosity ratio $\lambda$ for double emulsions stabilized by Na-caseinate with a non-gelled inner aqueous phase. Viscosity of $w_{2}$ was modified by addition of xanthan (closed circles) or pectin (closed squares). Error bars indicate standard deviation.

Larger oil droplet sizes in presence of low amounts of pectin (high viscosity ratio) indicate that the oil droplet size was not only due to a viscosity effect and that the droplet breakup may not have been determined by the viscous forces only. We hypothesize that this difference in oil droplet size is caused by changes in interfacial properties by adsorption of pectin or protein residues in pectin at the $\left(\mathrm{o} / \mathrm{w}_{2}\right)$ interface. Recently, the surface-active properties of pectin have been discussed, which have been attributed to a high degree of esterification [37]. A higher amount of esterified methyl groups increases the hydrophobicity of the polysaccharide, which favours adsorption at the $\left(\mathrm{o} / \mathrm{w}_{2}\right)$ interface through hydrophobic interactions [38]. Moreover, protein residues in pectin samples have also been associated with emulsifying properties for a long time [39-42]. The adsorption of pectin or protein residues at the interface, and possible interactions with hydrophilic emulsifiers may change the interfacial characteristics. For example, sugar beet pectin was previously found to pack less tightly compared to protein films at the air-water interface, causing formation of a less viscoelastic film [43]. Gülseren and Corredig [44] have shown that interfacial adsorption of pectin reduces the interfacial tension and influences the viscoelastic properties of the $\left(\mathrm{o} / \mathrm{w}_{2}\right)$ interface. The reported interfacial activity is in line with our observations. Such an influence on interfacial activity might lead to a reduced packing density of the hydrophilic emulsifiers, resulting in coalescence during or after emulsification. Such effects could explain the differences found. 
Further, oil droplet sizes decreased to $10 \mu \mathrm{m}$ for low viscosity ratios in the case of pectin, as shown in Figure 3.4. The change of droplet size for viscosity ratios lower than 5 is in contrast to double emulsions containing xanthan, for which the oil droplet breakup did not seem to be influenced below that viscosity ratio. Lower viscosity ratios for the emulsions with pectin were obtained, since pectin solutions remain viscous at higher shear rates. This leads to a higher degree of droplet breakup. As xanthan solutions are very shear thinning, the lower viscosities of the outer phase at higher shear rates lead to higher viscosity ratios, limiting oil droplet breakup.

To summarize, xanthan and pectin increase the viscosity of the outer water phase, thereby decreasing the viscosity ratio and facilitating oil droplet breakup. Depending on the type of thickener used, a decrease in oil droplet size is not only determined by the viscous forces, but might also be related to the interfacial properties by adsorption of the thickener. When adsorption of polysaccharides at oil-water interfaces occurs, we observed even smaller oil droplet sizes.

\subsubsection{Effect of thickeners on yield of inner water phase $w_{1}$ of double emulsions}

In the previous section, we have discussed the effect of thickener addition on the final oil droplet size of double emulsions. As yield is known to be related to oil droplet size (chapter $\mathbf{2}$ and [5]), we expected that the addition of thickeners would also influence the yield of double emulsions. For that reason, we discuss in this section the effect of thickener on the yield of double emulsions.

In Figure 3.5, yield versus viscosity ratio is shown for double emulsions stabilized by the three hydrophilic emulsifiers WPI, Tween 20 and Na-caseinate for double emulsions with gelled (G) or non-gelled (NG) inner aqueous phases. Double emulsions contained xanthan $(0-0.5 \mathrm{wt} \%)$ in the outer water phase to alter the viscosity.

It can be seen that yield decreased for all emulsions with decreasing viscosity ratio. Gelation of the inner water droplets had a positive effect on yield, particularly for protein-stabilized double emulsions. It seems that the change in yield as a function of viscosity ratio is similar for all hydrophilic emulsifiers, which implies that the expulsion of the droplets are driven by the viscous forces at the interface during droplet breakup. To get a better insight in the relation between droplet breakup and the yield of the emulsion, we considered the resulting oil droplet sizes. Figure $\mathbf{3 . 6}$ presents an overview of yield versus oil droplet sizes of all double emulsions stabilized with different emulsifiers with gelled (open symbols) or nongelled (filled symbols) inner water phases containing xanthan as a thickener in $\mathrm{w}_{2}$. Since osmotic pressure differences are suppressed by adjusting the osmotic gradient 


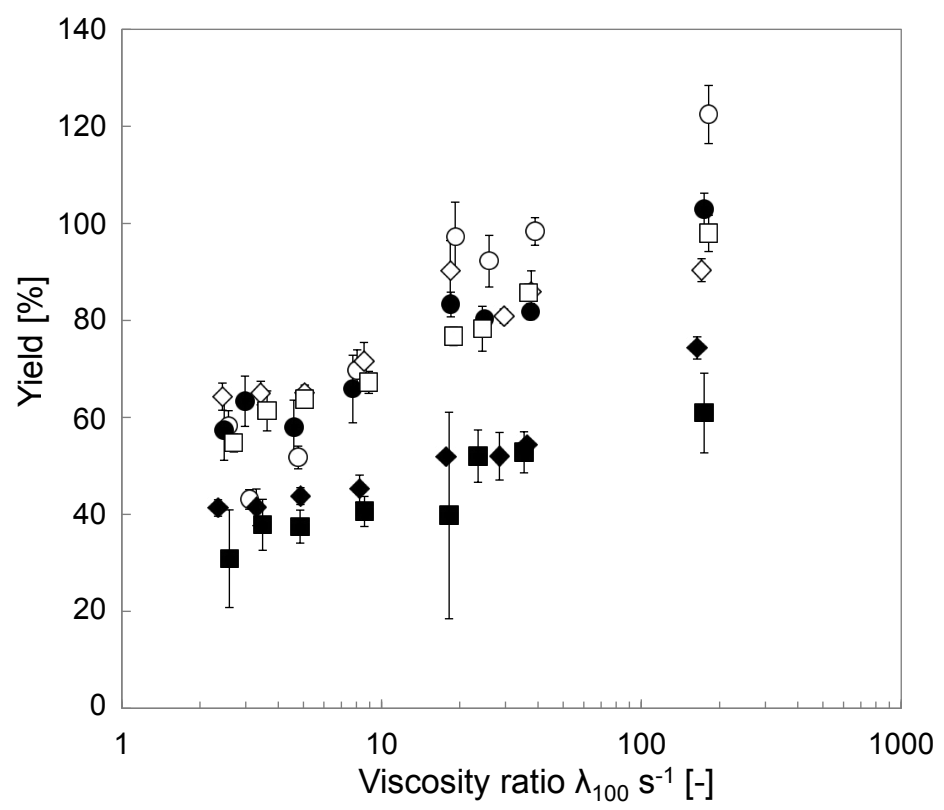

Figure 3.5 Yield as a function of viscosity ratio $\lambda$ for double emulsions with non-gelled (filled symbol) and gelled (open symbol) inner aqueous phase $w_{1}$. Viscosity ratio was modified by xanthan addition. Double emulsions were stabilized by WPI (diamonds), Tween 20 (circles), or Na-caseinate (squares). Error bars indicate standard deviation.

between the inner and outer water phase, we assume that diffusion effects are negligible. The observed differences are solely a result of the release of water by coalescence between the inner and outer water phase for the non-gelled emulsions and by expulsion of the inner water droplets for the emulsion with gelled droplets.

As can be seen from Figure 3.6, yields decreased for all double emulsions when oil droplets were smaller. This is as expected, and in agreement with chapter 2 and previous studies $[5,32,45,46]$. The smaller the oil droplets, the more inner water droplets get into contact with the outer oil-water interface, leading to more coalescence between the droplets and the outer water phase.

Yields were not only dependent on the oil droplet size, but also on the type of the hydrophilic emulsifier (proteins or surfactant), and the mechanical properties of the inner water droplets (gelled and non-gelled). When emulsifiers are compared, Tween 20-stabilized double emulsions (circles) showed the highest yields at all oil droplet sizes. Even at the smallest oil droplet size $(12 \mu \mathrm{m})$, yields were $43 \%$ for double emulsions with non-gelled water droplets and $63 \%$ for double emulsions with gelled water droplets. Protein-stabilized double emulsions, however, decreased to a minimum size of $20 \mu \mathrm{m}$ while only retaining $30 \%$ of the initially added water droplets. 
When yields of the two protein-stabilized double emulsions are compared, no differences between WPI and Na-caseinate (at the same oil droplet size) were found. It appears that once the viscosity of the outer water phase exceeds a certain value, the viscosity becomes more dominant and slight differences in interfacial properties between Na-caseinate and WPI become negligible.

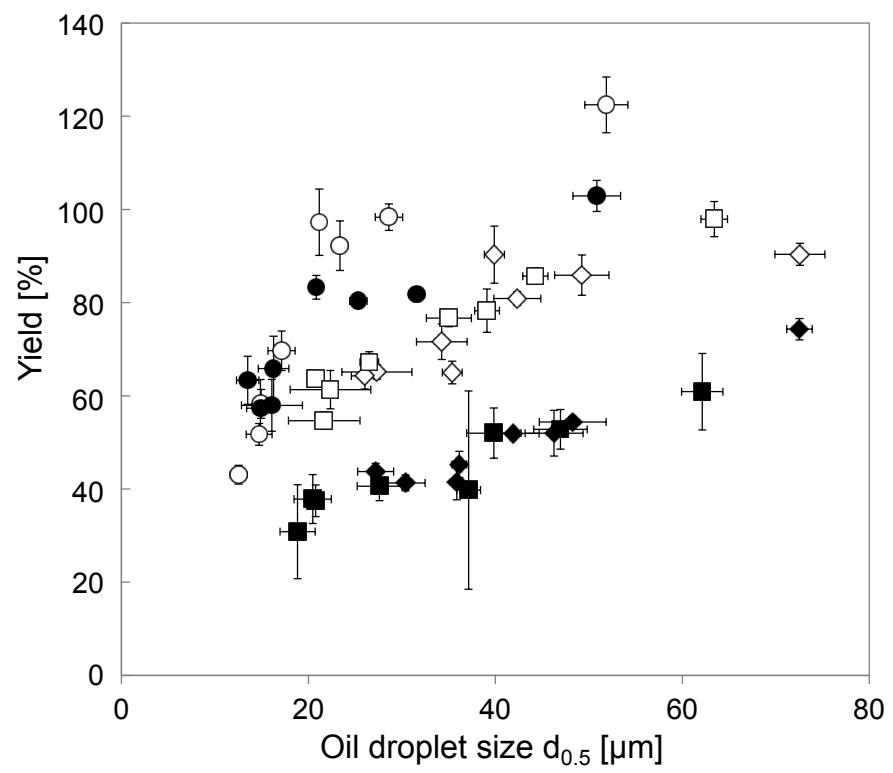

Figure 3.6 Yield versus oil droplet size for double emulsions with non-gelled (closed symbol) and gelled (open symbols) inner aqueous phase $w_{1}$. Oil droplet sizes were modified by varying viscosity ratios using xanthan. Double emulsions were stabilized by WPI (diamonds), Tween 20 (circles), or $\mathrm{Na}$-caseinate (squares). Error bars indicate standard deviation.

In addition to the type of hydrophilic emulsifier, also the mechanical properties of the inner water droplets influenced the yield. As already discussed in section 3.3.1, gelation of the inner aqueous phase had a positive effect on yield. Also at lower viscosity ratios, the gelation of the inner phase leads to higher yields, particularly for protein-stabilized double emulsions. In the case of Tween 20, the positive effect of gelation was decreased for higher viscosities, i.e. lower viscosity ratios.

These results show that in the case of higher viscosities, the viscous forces became dominant, and the effects of the interfaces became less relevant for the retention of $\mathrm{w}_{1}$ droplets in the oil droplets. As the higher viscous forces influenced the oil droplet size, the yield of double emulsions was also largely effected by a change in viscosity. 


\subsubsection{Effect of thickener type on yield of inner water phase $w_{1}$ of double emulsions}

In the previous section, we showed that the type of thickener has an effect on the droplet size due to possible changes in the interfacial characteristics. To investigate whether these changes also affect the yield of the emulsion, we have compared emulsions that were thickened by xanthan or pectin.

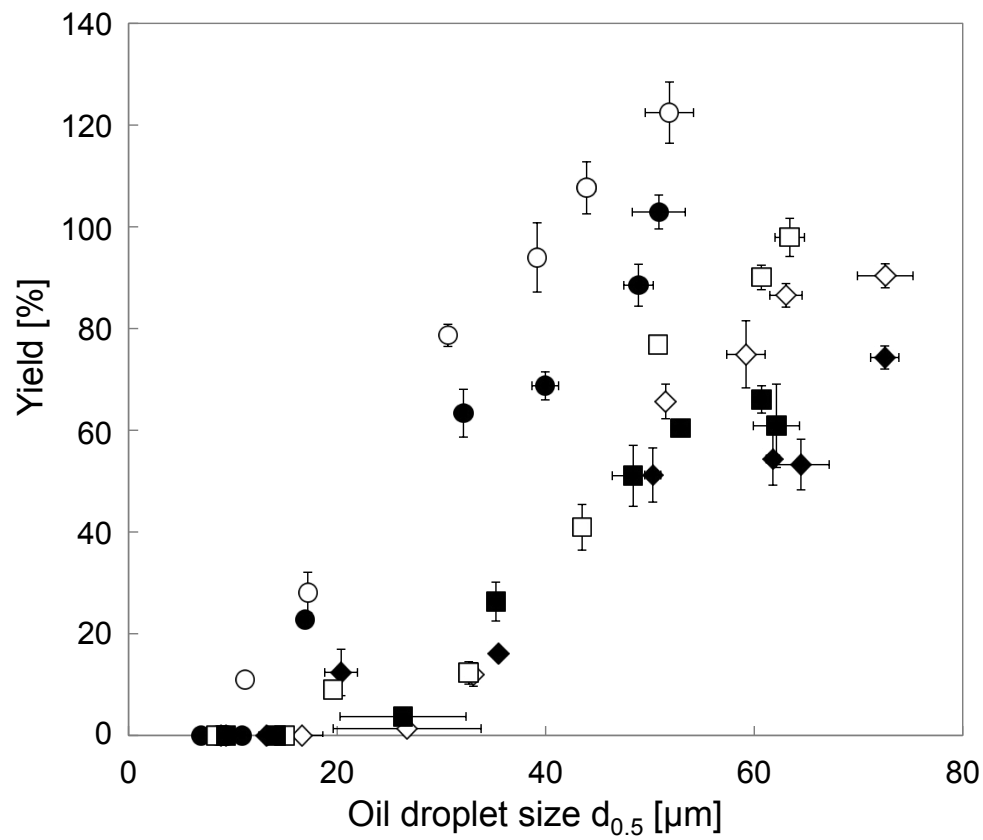

Figure 3.7 Yield as a function of oil droplet size for double emulsions with non-gelled (closed symbol) and gelled (open symbol) inner aqueous phase $w_{1}$. Oil droplet sizes were modified by varying viscosity ratios using pectin. Double emulsions were stabilized by WPI (diamonds), Tween 20 (circles), or $\mathrm{Na}$-caseinate (squares). Error bars indicate standard deviation.

As can be seen in Figure 3.7, yield also decreased in a linear fashion with decreasing oil droplet size when pectin was used as a thickener. In contrast to double emulsions containing xanthan, the gelation of the inner water droplets did not seem to have a positive effect on the yields. The decrease in yield with increasing pectin concentration was larger than for emulsions with xanthan, resulting in yields of $0 \%$ when oil droplets became smaller than $15 \mu \mathrm{m}$, for all types of emulsifier used. For both gelled and non-gelled conditions, all inner water droplets were expelled during emulsification, which was unexpected. Since (gelled) water droplets in our primary $\left(w_{1} / 0\right)$ emulsions are about $175 \mathrm{~nm}$ (chapter 2 ), oil droplet sizes of $15 \mu \mathrm{m}$ are theoretically large enough to hold a substantial amount of water droplets. However, at the time the DSC measurements were performed, the results indicated that oil 
droplets did not contain any inner water phase. This is partly in contrast to results found by Lutz and co-workers [14], who found that small oil droplets of $17 \mu \mathrm{m}$ had yields of $75 \%$ when the double emulsions contained $0.5 \%$ pectin and $4 \%$ WPI at $\mathrm{pH}$ 6. However, the concentration of pectin in their studies was much lower. While Lutz and co-workers used $0.5 \mathrm{wt} \%$, we used a much higher pectin concentration of $4 \mathrm{wt} \%$ in the outer water phase to obtain the same oil droplet size. Lower amounts of pectin possibly relates to less interactions with WPI at the interface, and therefore less changes in the interfacial characteristics.

To directly compare the results obtained for the two different thickeners, yields of double emulsions are given against the oil droplet size for emulsions with either xanthan or pectin in the outer water phase $\mathrm{w}_{2}$. As in section 3.3.2, we chose to discuss the data for emulsions stabilized with Na-caseinate only (Figure 3.8).

When directly comparing the yields of double emulsions with xanthan or pectin in the outer water phase, it can be seen that at oil droplet sizes smaller than $50 \mu \mathrm{m}$, double emulsions containing pectin had lower yields than double emulsions containing xanthan. This observation might be explained by changes in interfacial properties due to adsorption of pectin at the interface. We hypothesize that the adsorption of the thickeners competes with the adsorption of the protein, thereby leading to a less well-covered interface. This might have facilitated the expulsion of the droplets through the interface and led to lower yields. Schuch and co-workers [20] showed similar results. They investigated the yield of double emulsions for different stabilizers (pectin, carboxyl methylcellulose, gum arabic) in the absence of a hydrophilic emulsifier. They found that for polysaccharides able to adsorb to the interfaces, yields decreased. Highest yields were found for polysaccharides that did not adsorb at the interface. Higher yields for emulsions with surface-inactive stabilizers have also been described by others [47]. The ability of pectin or protein residues in pectin to adsorb at the interface leads to a competition with the hydrophilic emulsifiers at the interface, possibly leading to less unfolding and re-arrangement of proteins due to decreased mobility. This results in a thick but less tightly packed interface [48, 49]. Indeed, Ganzevles and co-workers [49] found that the presence of polysaccharides lowered protein adsorption compared to protein adsorption from a pure protein solution. We hypothesize that weak repulsive interactions between pectin and the emulsifiers inhibit emulsifier coverage at the interface, leading to a decreased packing density of proteins at the interface, which in turn facilitates a loss of inner water phase by coalescence with the outer water phase. 


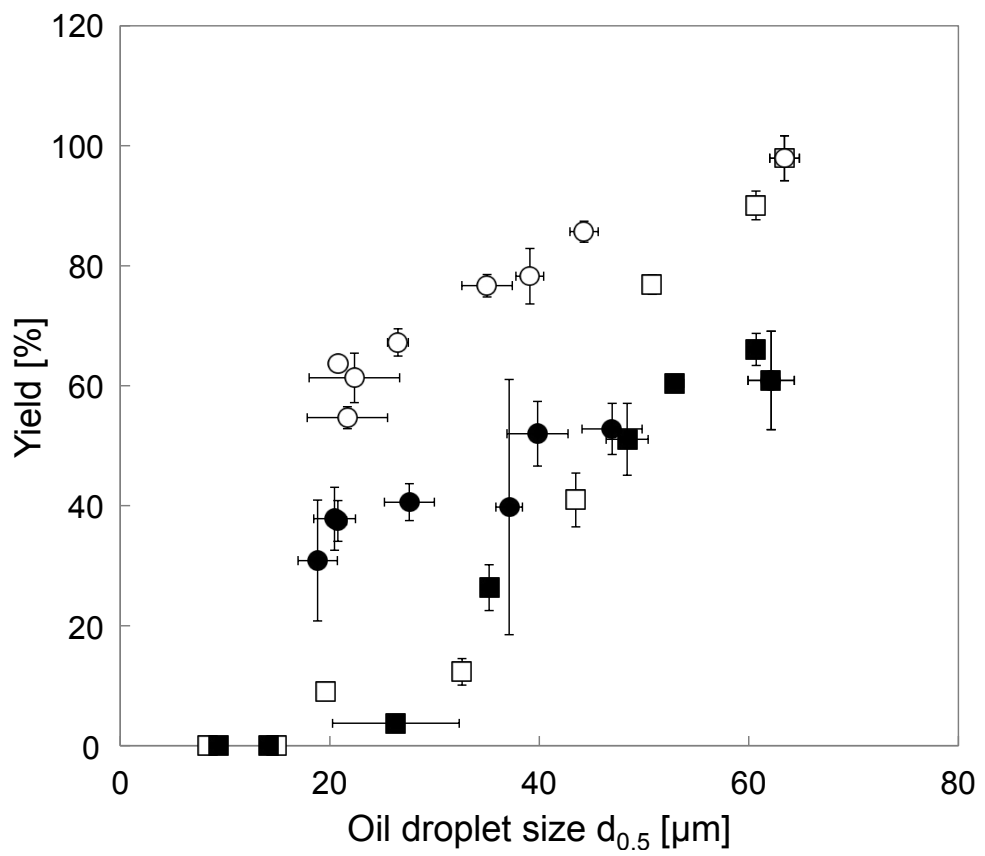

Figure 3.8 Yield versus oil droplet size for double emulsions stabilized by Na-caseinate with nongelled (closed symbol) and gelled (open symbol) inner aqueous phase $w_{1}$. Viscosity of $w_{2}$ was modified by addition of xanthan (circles) or pectin (squares). Error bars indicate standard deviation.

To conclude, the final oil droplet size and yield are determined by the viscosity of the continuous phase as well as interfacial properties. For very high viscosities, the strong viscous forces even promote expulsion of water droplets, and yields decreased. In combination with reduced emulsifier coverage, yields even decreased to $0 \%$ when pectin was used.

\subsection{Conclusions}

The aim of the present study was to investigate the effect of interfacial and bulk properties on the composition of double emulsions. Interfacial properties were altered by various hydrophilic emulsifiers and the bulk phase was varied in its viscosity through the use of different thickeners present in the outer water phase $w_{2}$. Both the oil droplet size and yield of $w_{1}$ phase of double emulsions were investigated.

We found that oil droplet size decreased and yields increased with the rate of adsorption of the hydrophilic emulsifiers at the oil-water interface. Oil droplet sizes decreased with increasing thickener addition due to reduced viscosity ratios. When comparing emulsions with HM pectin and xanthan as the thickener, oil droplets for emulsions with pectin were larger at the high viscosity ratio and decreased to lower 
sizes for low viscosity ratios. We attributed this difference to a reduced emulsifier coverage due to interactions between protein-residues and surface-active moieties in the pectin with the hydrophilic emulsifiers.

Yields decreased with decreasing oil droplet sizes, and differences between hydrophilic emulsifiers continued to exist. Yields for emulsions with pectin reduced to much lower values, due to the changes in the interfacial properties, which facilitated coalescence of $w_{1}$ with $w_{2}$. Gelation had a positive effect on yields due to reduced expulsion of $w_{1}$ droplets to the outer water phase.

Overall, adsorption kinetics of the emulsifier, mechanical properties of water droplets, viscosity ratio and surface-active properties of the thickener provide changes in both the bulk and interfacial characteristics to control double emulsion characteristics. While thickeners reduce creaming of emulsion droplets, they also influence oil droplet breakup in double emulsions. This knowledge can be used to design double emulsions as a fat replacer for different types of foods. It is important to consider environmental conditions and other ingredients in food applications to tailor double emulsion composition to obtain desired emulsion characteristics.

\section{Acknowledgements}

The research leading to these results has received funding from the European Union Seventh Framework Programme (FP7/2007-2013) under grant agreement nr. 289397 (TeRiFiQ project). The authors would like to thank Herman de Beukelaer and Willem Vogelzang for help with the DSC measurements. 


\section{References}

1. Muschiolik, G., Multiple emulsions for food use. Current Opinion in Colloid \& Interface Science, 2007. 12: p. 213-220.

2. Mezzenga, R., B.M. Folmer, and E. Hughes, Design of double emulsions by osmotic pressure tailoring. Langmuir, 2004. 20: p. 3574-3582.

3. Muguet, V., et al., Formulation of shear rate sensitive multiple emulsions. Journal of Controlled Release, 2001. 70: p. 37-49.

4. Pays, K., et al., Double emulsions: How does release occur? Journal of Controlled Release, 2002. 79: p. $193-205$.

5. Schuch, A., J. Wrenger, and H.P. Schuchmann, Production of W/O/W double emulsions. Part II: Influence of emulsification device on release of water by coalescence. Colloids and Surfaces A: Physicochemical and Engineering Aspects, 2013. 461: p. 344-351.

6. Pays, K., et al., Coalescence in surfactant-stabilized double emulsions. Langmuir, 2001. 17: p. 7758-7769.

7. Schuch, A., L.G. Leal, and H.P. Schuchmann, Production of W/O/W double emulsions. Part I: Visual observation of deformation and breakup of double emulsion drops and coalescence of the inner droplets. Colloids and Surfaces A: Physicochemical and Engineering Aspects, 2013. 461: 336-343.

8. Kanouni, M., H.L. Rosano, and N. Naouli, Preparation of a stable double emulsion (W1/O/W2): role of the interfacial films on the stability of the system. Advances in Colloid and Interface Science, 2002. 99: p. $229-254$.

9. Schmidts, T., et al., Influence of hydrophilic surfactants on the properties of multiple W/O/W emulsions. Journal of Colloid and Interface Science, 2009. 338: p. 184-92.

10. Chavez-Paez, M., et al., Coalescence in double emulsions. Langmuir, 2012. 28(14): p. 5934-5939.

11. Jiao, J. and D.J. Burgess, Rheology and stability of water-in-oil-in-water multiple emulsions containing Span 83 and Tween 80. AAPS PharmSci, 2003. 5: p. 1-12.

12. Ficheux, M.F., et al., Some stability criteria for double emulsions. Langmuir, 1998. 14: p. 2702-2706.

13. Garti, N., Double emulsions - scope, limitations and new achievements. Colloids and Surfaces A: Physicochemical and Engineering Aspects, 1997. 123-124: p. 233-246.

14. Lutz, R., et al., Double emulsions stabilized by a charged complex of modified pectin and whey protein isolate. Colloids and Surfaces B: Biointerfaces, 2009. 72: p. 121-127.

15. Schuch, A., K. Köhler, and H.P. Schuchmann, Differential scanning calorimetry (DSC) in multiple W/O/W emulsions. Journal of Thermal Analysis and Calorimetry, 2013. 111: p. 1881-1890.

16. Bos, M.A. and T. Van Vliet, Interfacial rheological properties of adsorbed protein layers and surfactants- a review. Advances in Colloid and Interface Science, 2001. 91: p. 437-471.

17. Gülseren, I. and M. Corredig, Interactions at the interface between hydrophobic and hydrophilic emulsifiers: Polyglycerol polyricinoleate (PGPR) and milk proteins, studied by drop shape tensiometry. Food Hydrocolloids, 2012. 29: p. 193-198.

18. Cofrades, S., et al., Preparation and impact of multiple (water-in-oil-in-water) emulsions in meat systems. Food Chemistry, 2013. 141: p. 338-346.

19. Benjamins, J., A. Cagna, and E.H. Lucassen-Reynders, Viscoelastic properties of triacylglycerol/ water interfaces covered by proteins. Colloids and Surfaces A: Physicochemical and Engineering Aspects, 1996. 114: p. 245-254.

20. Schuch, A., et al., Observations on the influence of different biopolymers on coalescence of inner water droplets in W/O/W (water-in-oil-in-water) double emulsions. Colloids and Surfaces A: Physicochemical and Engineering Aspects, 2014. 475: p. 2-8.

21. Dalgleish, D.G., M. Srinivasan, and H. Singh, Surface properties of oil-in-water emulsion droplets containing casein and Tween 60. Journal of Agricultural and Food Chemistry, 1995. 43: p. 2351-2355.

22. Dickinson, E. and M. Golding, Influence of calcium ions on creaming and rheology of emulsions containing sodium caseinate. Colloids and Surfaces A: Physicochemical and Engineering Aspects, 1998. 144: p. 167-177.

23. Hunt, J.A. and D.G. Dalgleish, Adsorption behaviour of whey protein isolate and caseinate in soya oil-in-water emulsions. Food Hydrocolloids, 1994. 8: p. 175-187.

24. Surh, J., et al., Preparation and characterization of water/oil and water/oil/water emulsions containing biopolymer-gelled waterdroplets. Journal of Agricultural and Food Chemistry, 2007. 55: p. $175-184$.

25. Janssen, J.M.H. and H.E.H. Meijer, Droplet breakup mechanisms: Stepwise equilibrium versus transient dispersion. Journal of Rheology, 1993. 37: p. 597-608.

26. McClements, D.J., Food emulsions: principles, practices, and techniques. 2nd ed. 2005, Boca Raton, FL: CRC Press. 
27. Bentley, B.J. and L.G. Leal, An experimental investigation of drop deformation and breakup in steady, two-dimensional linear flows. Journal of Fluid Mechanics, 1986. 167: p. 241-283.

28. Grace, H.P., Dispersion phenomena in high viscosity immiscible fluid systems and applications of static mixers as dispersion devices in such systems. Chemical Engineering Communications, 1982. 14: p. 225277.

29. Stroeve, P. and P.P. Varanasi, An experimental study on double emulsion drop breakup in uniform shear flow. Journal of Colloid and Interface Science, 1984. 99: p. 360-373.

30. Goubault, C., et al., Shear rupturing of complex fluids: Application to the preparation of quasimonodisperse water-in-oil-in-water double emulsions. Langmuir, 2001. 17: p. 5184-5188.

31. Leal-Calderon, F., et al., W/O/W emulsions with high internal droplet volume fraction. Food Hydrocolloids, 2012. 27: p. 30-41.

32. Schuch, A., et al., Production of W/O/W (water-in-oil-in-water) multiple emulsions: droplet breakup and release of water. Journal of Colloid and Interface Science, 2013. 402: p. 157-64.

33. Benichou, A., A. Aserin, and N. Garti, W/O/W double emulsions stabilized with WPI-polysaccharide complexes. Colloids and Surfaces A: Physicochemical and Engineering Aspects, 2007. 294: p. 20-32.

34. Kobori, T., A. Matsumoto, and S. Sugiyama, pH-Dependent interaction between sodium caseinate and xanthan gum. Carbohydrate Polymers, 2009. 75: p. 719-723.

35. Hemar, Y., et al., Influence of xanthan gum on the formation and stability of sodium caseinate oilin-water emulsions. Food Hydrocolloids, 2001. 15: p. 513-519.

36. Schmidt, U.S., et al., Influence of the degree of esterification on the emulsifying performance of conjugates formed between whey protein isolate and citrus pectin. Food Hydrocolloids, 2016. 56: p. 1-8.

37. Schmidt, U.S., L. Schütz, and H.P. Schuchmann, Interfacial and emulsifying properties of citrus pectin: Interaction of $\mathrm{pH}$, ionic strength and degree of esterification. Food Hydrocolloids, 2017. 62: p. 288-298.

38. Falk, J.D. and J.J. Nagyvary, Exploratory studies of lipid-pectin interactions. The Journal of nutrition, 1982. 112: p. 182-188.

39. Ngouémazong, E.D., et al., The Emulsifying and Emulsion-Stabilizing Properties of Pectin: A Review. Comprehensive Reviews in Food Science and Food Safety, 2015. 14: p. 705-718.

40. Leroux, J., et al., Emulsion stabilizing properties of pectin. Food Hydrocolloids, 2003. 17: p. 455-462.

41. Dickinson, E., Hydrocolloids at interfaces and the influence on the properties of dispersed systems. Food Hydrocolloids, 2003. 17: p. 25-39.

42. Akhtar, M., et al., Emulsion stabilizing properties of depolymerized pectin. Food Hydrocolloids, 2002. 16: $\mathrm{p}$. 249-256.

43. Gromer, A., et al., Interfacial structure of sugar beet pectin studied by Atomic Force Microscopy. Langmuir, 2009. 25: p. 8012-8018.

44. Gülseren, I. and M. Corredig, Interactions between polyglycerol polyricinoleate (PGPR) and pectins at the oil-water interface and their influence on the stability of water-in-oil emulsions. Food Hydrocolloids, 2014. 34: p. 154-160.

45. Hattrem, M.N., et al., Macro- vs. micromolecular stabilisation of W/O/W-emulsions. Food Hydrocolloids, 2014. 37: p. 77-85.

46. Muguet, V., et al., W/O/W multiple emulsions submitted to a linear shear flow: Correlation between fragmentation and release. Journal of Colloid and Interface Science, 1999. 218: p. 335-337.

47. Bouyer, E., et al., Proteins, polysaccharides, and their complexes used as stabilizers for emulsions: Alternatives to synthetic surfactants in the pharmaceutical field? International Journal of Pharmaceutics, 2012. 436: p. 359-378.

48. Dickinson, E., Mixed biopolymers at interfaces: Competitive adsorption and multilayer structures. Food Hydrocolloids, 2011. 25: p. 1966-1983.

49. Ganzevles, R.A., et al., Structure of mixed $\beta$-lactoglobulin/pectin adsorbed layers at air/water interfaces; a spectroscopy study. Journal of Colloid and Interface Science, 2008. 317: p. 137-147. 


\section{Chapter 4}

\section{Influence of double $\left(w_{1} / 0 / w_{2}\right)$ emulsion composition on lubrication properties}
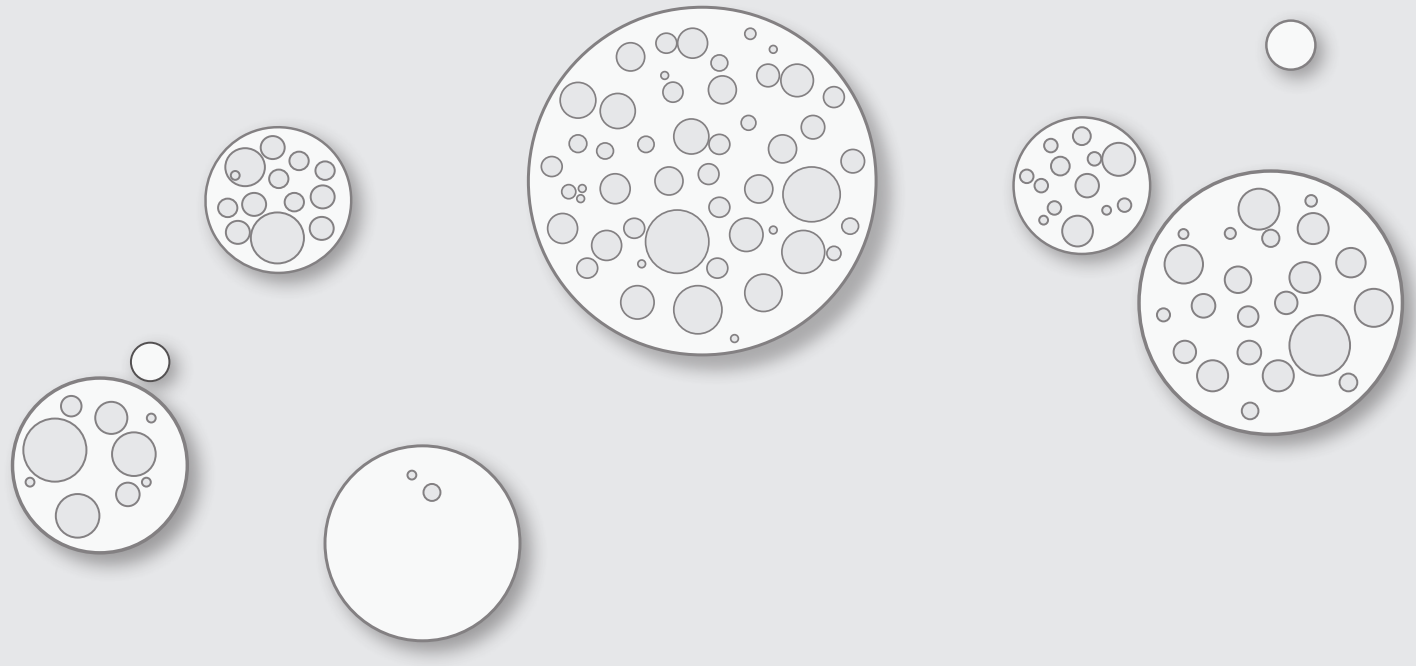

This chapter has been published as

AKL Oppermann, LC Verkaaik, M Stieger, E Scholten, Influence of double $\left(w_{1} / o / w_{2}\right)$ emulsion composition on lubrication properties, Food \& Function (2016), DOI: 10.1039/ C6FO01523A 


\section{Abstract}

Double $\left(w_{1} / 0 / w_{2}\right)$ emulsions are potential fat replacers in foods. Fats are known for its lubricating properties, which contribute to texture perception. It is therefore of interest to understand how the composition of double emulsions influences lubrication properties. This study focuses on the understanding of the influence of the fraction of inner dispersed aqueous phase $w_{1}$ and the gelation of the $w_{1}$ droplets on the lubrication properties of double emulsions.

The addition of an inner water phase $w_{1}$ to the oil droplets decreased friction at low entrainment speeds due to adsorption of the lipophilic emulsifier polyglycerol polyricinoleate (PGPR) at the hydrophobic tribo-surface. At higher entrainment speeds, double emulsions with $\mathrm{w}_{1}$ fractions of up to $6 \%$ (corresponding to fat reduction of $20 \%$ ) displayed comparable tribological behaviour as full-fat single $\left(\mathrm{o} / \mathrm{w}_{2}\right)$ emulsions. For double emulsions with gelled $\mathrm{w}_{1}$ droplets at higher $\mathrm{w}_{1}$ fractions of up to $15 \%$ (corresponding to fat reduction of $50 \%$ ), an increase in friction was observed compared to full-fat single $\left(\mathrm{o} / \mathrm{w}_{2}\right)$ emulsions. The increase in friction is probably related to the presence of gelled droplets expelled from the inner $w_{1}$ into the outer $w_{2}$ phase, and to the deformability of $\left(w_{1} / 0\right)$ droplets. Lubrication decreased when gelled particles were expelled from the inner $w_{1}$ phase to the outer $w_{2}$ phase. Lubrication also decreased when the deformability of ( $\left.w_{1} / 0\right)$ droplets decreased, since less deformable $\left(w_{1} / 0\right)$ droplets spread less easily on the tribo-pair surface. Knowledge about lubrication properties of double emulsions can be used in future studies to relate composition to sensory perception and develop double emulsions further as fat replacers. 


\subsection{Introduction}

Emulsions and emulsion-based foods represent an interesting food category for fat reduction due to the often high amounts of dispersed fats and oils in those products. A potential approach for fat reduction is the use of double $\left(w_{1} / o / w_{2}\right)$ emulsions. Double emulsions are complex multiphase systems, in which small water droplets are entrapped inside larger oil droplets $\left(w_{1} / 0\right)$, which are subsequently dispersed in a water-continuous phase, $w_{2}$. Consequently, at a given oil droplet size the oil droplet surface area remains similar to those of $\left(\mathrm{o} / \mathrm{w}_{2}\right)$ single emulsions, while the amount of oil is decreased.

Oil droplets in emulsion-based foods contribute to the perception of certain sensory and mouthfeel attributes, such as creaminess and fattiness. Sensory perception of emulsions is influenced by rheological and tribological properties during different stages of oral processing [1, 2]. The amount of fat in an emulsion can greatly affect the lubrication of oral surfaces during consumption, and can therefore have a positive influence on sensory perception.

Several authors studied the lubrication properties of single oil-in-water $\left(\mathrm{o} / \mathrm{w}_{2}\right)$ emulsions. Generally, increasing oil content of single $\left(\mathrm{o} / \mathrm{w}_{2}\right)$ emulsions decreased friction coefficients by reducing close contact between the surfaces of the tribopair [3-10]. However, when oil content in single emulsions exceeded a certain level (15 to $30 \%$ ), no differences in lubrication behaviour were found anymore between emulsions varying in oil volume fraction [5]. It was suggested that friction is mostly affected by oil volume fraction at lower oil volume fractions, i.e. below $15 \%$. Oil droplets are deformable and can provide lubrication between surfaces by forming a film or oil film patches. Furthermore, the solid fat content (SFC) of oils has been shown to influence lubrication. In emulsion-filled gels, Liu and co-workers [11] found that friction decreased with increasing SFC due to partial coalescence of fat crystals, even though fat droplets with higher SFC are less deformable. Oil droplet size of emulsions also has been shown to influence friction. De Wijk and co-workers [12] showed that increasing oil droplet size (up to $6 \mu \mathrm{m}$ ) in mayonnaise increased friction, indicating that smaller droplets have a larger effect on lubrication. Even though the effect of fat content of single emulsions on lubrication properties has been studied, the effect of the type of emulsifier on lubrication properties of single emulsions received less attention. It has been reported that low molecular weight surfactants such as sucrose esters or Tween 20 decrease friction in single emulsions with high oil content $[2,13]$. Overall, the composition of single (o/ $\mathrm{w}_{2}$ ) emulsions (fat content, oil droplet size, type of emulsifier) have an influence on the lubrication behaviour. 
While the lubrication properties of single emulsions have been studied, to the best of our knowledge, the lubrication properties of double $\left(w_{1} / o / w_{2}\right)$ emulsions have not been investigated. Double emulsions differ in various aspects from single emulsions. First, oil droplets in double emulsions are typically larger than in single emulsions to retain water droplets inside. While the diameter of oil droplets in single emulsions is typically of the order of 1-10 $\mu \mathrm{m}$, the diameter of oil droplets in double emulsions is typically larger than $50 \mu \mathrm{m}$. Second, small water droplets, typically smaller than $1 \mu \mathrm{m}$, are dispersed into larger oil droplets. The inclusion of water droplets increases the viscosity of the oil droplets, and consequently decreases the deformability of the oil droplets. Third, double emulsions are prepared with two surfactants. The inner water droplets are stabilized by a lipophilic surfactant, often polyglycerol polyricinoleate (PGPR), and the outer phase usually by proteins such as whey protein isolate (WPI) or $\mathrm{Na}$-caseinate. To increase the stability of double emulsions, the inner water droplets $\mathrm{w}_{1}$ can be gelled, which results in the retention of $\mathrm{w}_{1}$ droplets inside the oil droplets. Not much is known about the lubrication behaviour of complex multiphase emulsions and how the lubrication properties are affected by their composition.

The aim of this study was to investigate the tribological properties of double $\left(w_{1} / o / w_{2}\right)$ emulsions varying in composition. Specifically, the influence of inner dispersed water phase $w_{1}$ fraction, dispersed oil phase fraction, and the effect of lipophilic emulsifier PGPR on the tribological properties were studied. Knowledge about lubrication properties of double emulsions can be used in future studies to relate emulsion composition to sensory perception and develop double emulsions as effective fat replacers.

\subsection{Materials and methods}

\subsubsection{Materials}

Whey protein isolate (WPI, BiPro JE-099-2-420) from Davisco Foods International Inc. (Le Sueur, MN) was used as hydrophilic emulsifier. The composition of WPI as stated by the manufacturer was $97.7 \%$ protein, $0.3 \%$ fat, $1.8 \%$ ash (dry weight basis). $\mathrm{NaCl}$ was obtained from Sigma-Aldrich (purity $\geq 99.5 \%$, Sigma-Aldrich, Steinheim, Germany). Gelatin (type A, Type 250 PS 30) from Rousselot (Gent, Belgium) was used. Commercial sunflower oil was purchased from a local retailer (Wageningen, the Netherlands). Polyglycerol polyricinoleate (PGPR 4175) was kindly provided by Palsgaard (Juelsminde, Denmark). For all aqueous solutions, purified water (Milli-Q, $18.2 \mathrm{M} \Omega \mathrm{cm}$ at $25^{\circ} \mathrm{C}$ ) was used. 


\subsubsection{Preparation of emulsions}

\subsubsection{Preparation of solutions}

For the inner aqueous phase $\mathrm{w}_{1}, \mathrm{NaCl}$ solutions were prepared by dissolving $0.4 \mathrm{wt} \%$ $\mathrm{NaCl}$ in purified water while stirring. Gelatin solutions were prepared by dissolving $10 \mathrm{wt} \%$ gelatin and $0.4 \mathrm{wt} \% \mathrm{NaCl}$ in purified water at $60^{\circ} \mathrm{C}$ while stirring. The solutions were kept at $60^{\circ} \mathrm{C}$ until the gelatin was completely dissolved. Gelatin was used to gel the inner water droplets in some of the emulsions. The oil phase was prepared by adding PGPR ( 0 to $9 \mathrm{wt} \%$ ) to sunflower oil followed by $30 \mathrm{~min}$ stirring at $20^{\circ} \mathrm{C}$. The outer aqueous phase $\mathrm{w}_{2}$ was prepared by adding $0.2 \mathrm{wt} \% \mathrm{NaCl}$ and $1 \mathrm{wt} \% \mathrm{WPI}$ to purified water while stirring. Stirring was continued for $2 \mathrm{~h}$ at $20^{\circ} \mathrm{C}$. Different concentrations of $\mathrm{NaCl}$ were required to adjust the osmotic pressure - Laplace pressure balance, as reported in chapter 2 . All solutions were stored at $4{ }^{\circ} \mathrm{C}$ overnight before use.

\subsubsection{Preparation of $\left(w_{1} / 0\right)$ emulsions}

Primary water-in-oil $\left(\mathrm{w}_{1} / \mathrm{o}\right)$ emulsions were prepared by adding between 10 and $50 \mathrm{wt} \%$ of aqueous phase $\mathrm{w}_{1}$ to the oil phase in a $1000 \mathrm{~mL}$ stainless steel container of a Waring blender 8011 ES (Stamford, CT), and mixing at 22000 rpm for 60 s. As reported in chapter 5, the maximum inner dispersed phase content was $30 \mathrm{wt} \%$. To achieve higher fat reduction levels of 40 and $50 \mathrm{wt} \%$, gelation of the inner water droplets was necessary. For double emulsions with gelatin in the inner aqueous phase, both the oil phase and the gelatin solution were heated to $60^{\circ} \mathrm{C}$ in a water bath prior to mixing. A premixing step was used to add 40 or 50 wt $\%$ of the heated gelatin solution to the oil phase while increasing the mixing speed of a high speed blender (Ultra Turrax T25 with the dispersing tool S25-N 18G, IKA, Staufen, Germany) from 3000 rpm to $8000 \mathrm{rpm}$ within $1 \mathrm{~min}$ and mixed at $8000 \mathrm{rpm}$ for $4 \mathrm{~min}$. The primary emulsion was then transferred to the Waring blender and mixed at $22000 \mathrm{rpm}$ for $60 \mathrm{~s}$. Samples were subsequently cooled under tap water for at least 15 min to allow gelation of the inner water droplets. Water droplet sizes $\left(d_{0.5}\right)$ of the different primary $\left(w_{1} / o\right)$ emulsions were similar and ranged from 150-250 $\mathrm{nm}$ with all droplets being smaller than $1 \mu \mathrm{m}$ (Mastersizer 2000, Malvern Instruments, Worcestershire, UK), as reported in chapter 2 and 5.

\subsubsection{Preparation of single $\left(0 / w_{2}\right)$ and double $\left(w_{1} / o / w_{2}\right)$ emulsions}

Double emulsions were prepared by dispersing $30 \mathrm{wt} \%$ of the primary $\left(\mathrm{w}_{1} / \mathrm{o}\right)$ emulsion into $70 \mathrm{wt} \%$ of outer aqueous phase $\mathrm{w}_{2}$ while increasing the mixing speed of the high speed blender (Ultra Turrax T25 with the dispersing tool S25-N 18G, IKA, 
Staufen, Germany) from $3000 \mathrm{rpm}$ to the required speed within one minute. Mixing was continued for $4 \mathrm{~min}$ at the required speed. The mixing speed was adapted for each formulation to obtain similar oil droplet size distributions for all emulsions. All emulsions had broad, monomodal size distributions (ranging from about 10 to $100 \mu \mathrm{m}$ ) with average oil droplet sizes $\left(\mathrm{d}_{0.5}\right)$ of $50 \pm 5 \mu \mathrm{m}$ (Mastersizer 2000, Malvern Instruments, Worcestershire, UK).

Single oil-in-water $\left(\mathrm{o} / \mathrm{w}_{2}\right)$ emulsions were prepared by dispersing $30 \mathrm{wt} \%$ of oil phase (with or without PGPR) in $70 \mathrm{wt} \%$ of outer water phase $w_{2}$ within one minute in a high speed blender (Ultra Turrax T25 with the dispersing tool S25-N 18G, IKA, Staufen, Germany), while increasing the speed from $3000 \mathrm{rpm}$ to the required speed, after which mixing was continued for $4 \mathrm{~min}$.

For some single ( $\left.\mathrm{o} / \mathrm{w}_{2}\right)$ emulsions, gelled particles (with gelatin as a gelling agent) were added to the outer water phase $w_{2^{\prime}}$ to investigate the effect of gelled water droplets expelled from the inner $w_{1}$ to the outer $w_{2}$ water phase. To prepare the gelled particles, a $10 \mathrm{wt} \%$ water-in-oil ( $\left.\mathrm{w}_{1} / \mathrm{o}\right)$ emulsion containing a $10 \mathrm{wt} \%$ gelatin solution was prepared as described in section 4.2.2.2. The $\left(w_{1} / 0\right)$ emulsion was centrifuged for $90 \mathrm{~min}$ at $25000 \mathrm{rpm}(74600 \mathrm{rcf})$ at $15^{\circ} \mathrm{C}$ using a high performance centrifuge (Avanti J-26 XP, rotor JA-25.50, Beckham Coulter, Brea, US) to separate the gelled particles from the oil phase. The pellet of gelled particles was washed with a $1 \mathrm{wt} \%$ WPI solution at a ratio of 1:2 and the mixture was mixed using a high speed blender (Ultra Turrax T25 with the dispersing tool S25-N 18G, IKA, Staufen, Germany) for 5 min at $1200 \mathrm{rpm}$. WPI was added to aid the removal of the oil and the excess emulsifier. The mixture was then homogenized (Lab-scale homogenizer, Delta instruments, Drachten, the Netherlands) for six times at 150 bar. The sample was then centrifuged again under the same conditions for $60 \mathrm{~min}$. The pellet was removed after this second centrifugation step. Fractions of this pellet ( 2.5 and $5 \mathrm{wt} \%$ ) were added to different single $\left(o / w_{2}\right)$ emulsions to investigate the effect of the presence of particles in the outer water phase $\mathrm{w}_{2}$.

An overview of the composition and characteristics of all single $\left(\mathrm{o} / \mathrm{w}_{2}\right)$ and double $\left(w_{1} / o / w_{2}\right)$ emulsions can be found in Table 4.1. Sample names indicate the emulsion type ("OW" for single emulsions, "WOW" for double emulsions), the amount of inner aqueous phase $\mathrm{w}_{1}$, oil phase and outer water phase $\mathrm{w}_{2}$ in the final emulsion, followed by the physical state of the inner aqueous phase ("NG" for non-gelled, "G" for gelled). For example, the label "WOW 3-27-70 NG" refers to a double emulsion containing $3 \mathrm{wt} \%$ of non-gelled $\mathrm{w}_{1}$ droplets dispersed in $27 \mathrm{wt} \%$ of oil, further dispersed in $70 \mathrm{wt} \%$ of outer continuous water phase $\mathrm{w}_{2}$. The $3 \mathrm{wt} \%$ of inner aqueous phase $\mathrm{w}_{1}$ 


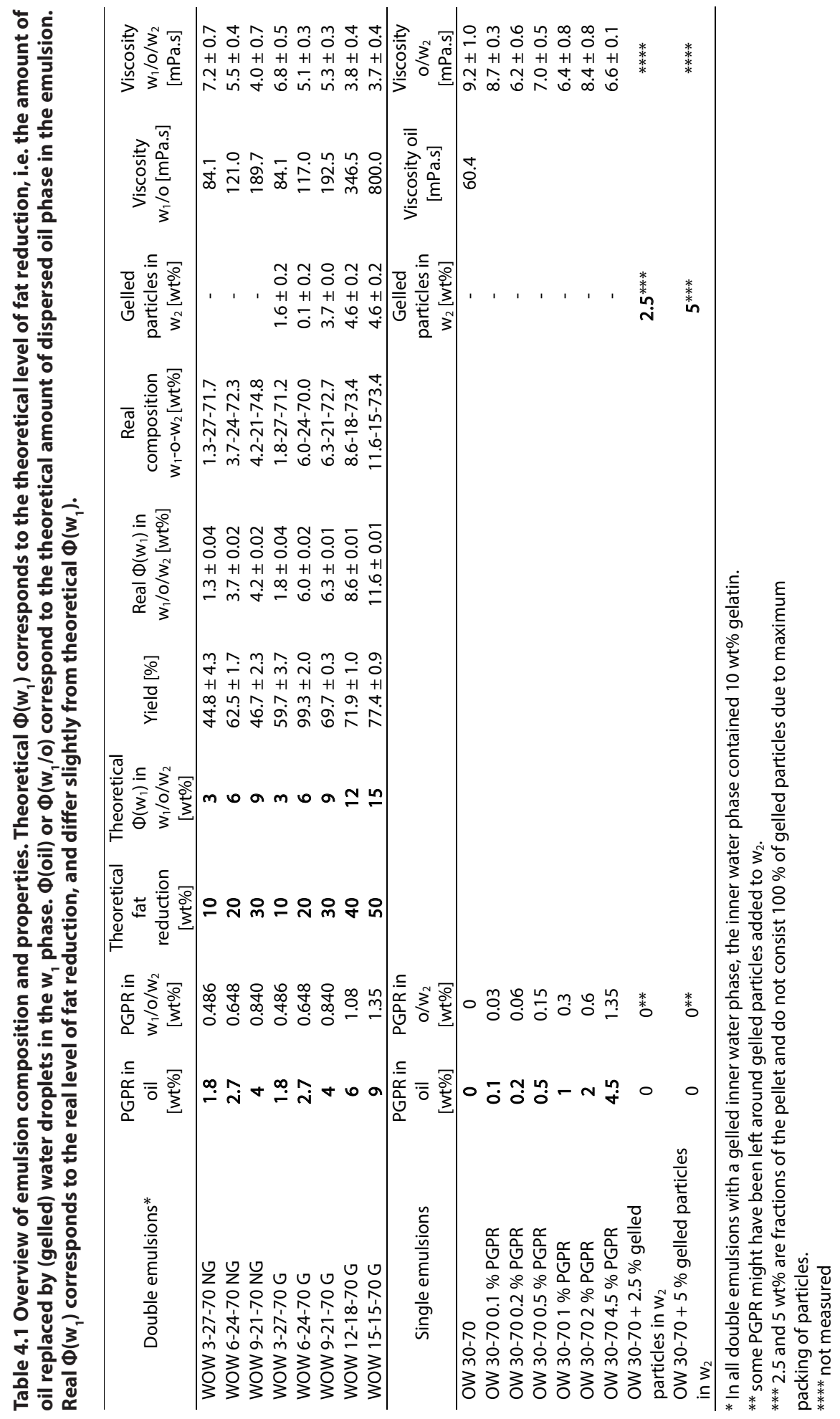


and $27 \mathrm{wt} \%$ of oil correspond to a total of $30 \mathrm{wt} \%$ of dispersed $\left(w_{1} / 0\right)$ phase. Since a small fraction of the inner water droplets is expelled to the outer water phase during preparation, the real mass fractions of the two aqueous phases are slightly different. The real dispersed phase $\left(w_{1} / o\right)$ fractions were determined by measuring the yield, i.e. the amount of $w_{1}$ droplets remaining inside the oil droplets after emulsion preparation (see section 4.2.3.4). The real dispersed phase mass fractions are listed in Table 4.1.

\subsubsection{Emulsion characterization}

\subsubsection{Viscosity measurements of emulsions}

Viscosity measurements of single $\left(w_{1} / 0\right),\left(0 / w_{2}\right)$ and double $\left(w_{1} / 0 / w_{2}\right)$ emulsions were performed with a Modular Compact Rheometer 301 (MCR 301, Anton Paar, Graz, Austria). The geometry used was a concentric cylinder (CC17/Ti-SN3960). Measurements were performed at $20^{\circ} \mathrm{C}$ with a sample volume of $4.7 \mathrm{~mL}$, with an increasing shear rate from $0.01 \mathrm{~s}^{-1}$ to $1000 \mathrm{~s}^{-1}$ within $17 \mathrm{~min}$. Each emulsion was measured in duplicate. Since emulsions were only slightly shear-thinning (maximum difference in viscosity of $2 \mathrm{mPa}$.s between shear rates of $10 \mathrm{~s}^{-1}$ and $\left.1000 \mathrm{~s}^{-1}\right)$, average viscosities were calculated over a range of shear rates $\left(10-100 \mathrm{~s}^{-1}\right.$ ) (see Table 4.1).

\subsubsection{Friction measurements}

Lubrication properties of double emulsions were determined using a commercially available tribology cell (T-PTD 200-SN81766963) attached to a stress-controlled rotational shear rheometer (MCR 301, Anton Paar, Graz, Austria). Friction coefficients were measured on a glass ball-on-three-pins setup, as depicted in Figure 4.1. The setup consists of a glass ball ((BC12.7-SN40500, diameter $12.7 \mathrm{~mm})$ and three polydimethylsiloxane (PDMS) pins (diameter of $6 \mathrm{~mm}$, roughness $0.2 \mu \mathrm{m} \pm 0.03$ ), which are inserted into the conical measuring cell. Before lowering the glass ball into the measuring position, $0.6 \mathrm{~mL}$ of freshly prepared emulsion was loaded by pipetting in the measuring cell. The friction coefficient was measured while varying the rotational speed of the glass ball from 0.1 to $1000 \mathrm{rpm}$ (corresponding to $0.47 \mathrm{~mm} / \mathrm{s}$ to $470 \mathrm{~mm} / \mathrm{s}$ ) in $4.3 \mathrm{~min}$, followed by $4.3 \mathrm{~min}$ during which the rotational speed decreased from $1000 \mathrm{rpm}$ to $0.1 \mathrm{rpm}$. Friction coefficients at a rotation speed below $10 \mathrm{rpm}$ were neglected due to large variations in friction coefficients between measurements at those low speeds. A constant normal load of $1 \mathrm{~N}$ was applied and the temperature was set at $20^{\circ} \mathrm{C}$. All measurements were performed in triplicate with three independent runs, for each of which a new sample of the same emulsion was loaded in the measurement cell. New PDMS probes were used for each measurement. 
Friction coefficients are reported as a function of entrainment speed multiplied by the average viscosity of the particular emulsion. The entrainment speed was multiplied by the average viscosity of each emulsion formulation to take into account small differences in viscosity.

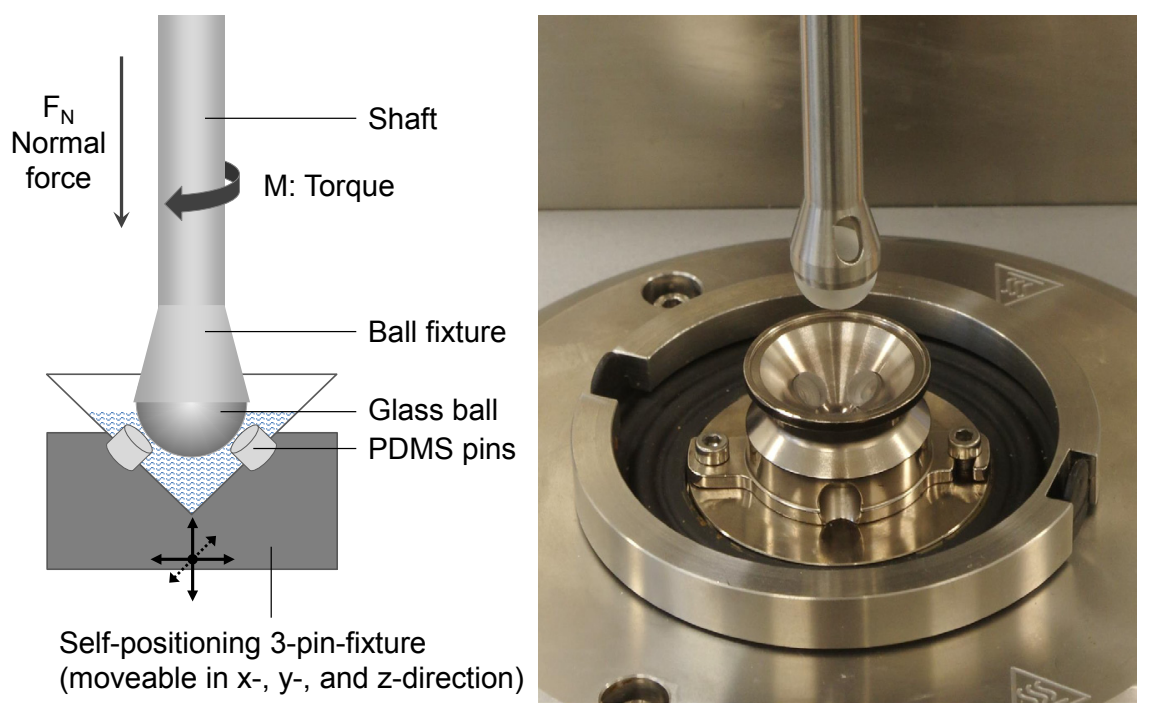

Figure 4.1 Schematic representation and picture of tribometer setup.

\subsubsection{Oil droplet size measurements}

Oil droplet sizes of all double $\left(w_{1} / 0 / w_{2}\right)$ emulsions were measured by static light scattering with a Mastersizer 2000 (Malvern Instruments, Worcestershire, UK). The refractive index of the water-containing oil droplets was set at 1.45 and the one of demineralized water (continuous phase) at 1.33. Absorption was set at 0.01 . The size distribution of each sample was measured three times. Volume-based average sizes are reported as $d_{0.5}$.

\subsubsection{Determination of inner water phase fraction in double emulsions}

DSC measurements were carried out to determine the amount of inner water droplets still present within the oil droplets after emulsion preparation, also known as the yield. Samples between 7 and $12 \mathrm{mg}$ were hermetically sealed in an aluminum pan and introduced into the calorimeter (Diamond DSC, Perkin Elmer, Pyris, USA). After equilibration at $10^{\circ} \mathrm{C}$ for $1 \mathrm{~min}$, samples were cooled to $-60^{\circ} \mathrm{C}$ at a cooling rate of $5^{\circ} \mathrm{C}$ per min. For each double emulsion formulation, the corresponding $\left(w_{1} / 0\right)$ emulsion was measured as a reference for a yield of $100 \%$. Each duplicate of each double emulsion was measured twice. A detailed description of the calculation of the yield has been reported in chapter 2 . 
Based on the yield, real inner dispersed phase fractions $\varphi_{\mathrm{w} 1 \text {,real }}$ (weight-based) were calculated:

$$
\varphi_{w_{1, \text { real }}}=\text { yield } * \varphi_{w_{1, \text { theoretical }}}
$$

In case of non-gelled $w_{1}$ droplets, coalescence of those water droplets with the outer water phase only leads to a slight decrease in the dispersed phase fraction. The loss of gelled $w_{1}$ droplets, however, leads to the presence of gelled particles in the outer water phase, as shown in Table 4.1. Due to these changes during the emulsion preparation, the real fractions for both the inner water droplets and the outer water phase slightly deviate from the theoretical fractions. The real weight fractions are given in Table 4.1.

\subsection{Results and discussion}

\subsubsection{Influence of inner water phase fraction on lubrication behaviour of double emulsions}

To investigate the influence of inner water droplets $w_{1}$ within the oil droplets of the double $\left(w_{1} / o / w_{2}\right)$ emulsions on lubrication properties, friction coefficients of double emulsions containing various amounts of inner water phase fractions are shown in Figure 4.2. Fat reduction was varied by replacing 10, 20, or $30 \mathrm{wt} \%$ of the oil with small water droplets. Since the $w_{1}$ droplets were not gelled, the maximum possible amount of added droplets is limited. For comparison, the friction coefficient of the corresponding full-fat single $\left(\mathrm{o} / \mathrm{w}_{2}\right)$ emulsion is shown in the same figure. All emulsions had a total dispersed $\left(w_{1} / 0\right)$ fraction of $30 \mathrm{wt} \%$.

As can be seen in Figure 4.2, all double emulsions were characterized by low friction coefficients at low entrainment speeds, followed by an increase in friction coefficient up to an inflection point before friction coefficients decreased again. First, we discuss the differences between the friction curves of all double emulsions, followed by a discussion of those curves in comparison to the single $\left(0 / w_{2}\right)$ emulsion.

For double emulsions with higher inner water phase fractions ( 6 and 9 wt\% of the double emulsion, corresponding to 20 and 30 wt $\%$ fat reduction ${ }^{1}$ ), the increase in friction coefficient was more pronounced. Those double emulsions had slightly higher friction coefficients than the double emulsion with $3 \mathrm{wt} \%$ inner water phase (corresponding to $10 \%$ fat reduction). We do not have an explanation for the lower friction at very low entrainment speeds of the double emulsion with $3 \%$ inner water phase. Even though error bars are large, it seems that a higher inner water phase

1 Due to loss of inner water droplets during emulsification, real $w_{1}$ fractions were very similar (3.7 and $4.2 \mathrm{wt} \%$ ) 
fraction tended to lead to an increase in friction. The increased number of small water droplets increased the viscosity of the dispersed $\left(w_{1} / o\right)$ droplets, resulting in less deformable $\left(w_{1} / o\right)$ droplets compared to when less water droplets were present in the oil. When dispersed $\left(w_{1} / o\right)$ droplets are less deformable, they cannot enter the gap easily and spread less easily on the tribo-surfaces to provide lubrication. Consequently, an increased friction was observed with increasing inner water phase fraction. The effect of an increased friction by an increase in particle stiffness has been described before [8]. As the entrainment speed increased, dispersed $\left(w_{1} / 0\right)$ droplets might enter the gap, and the surfaces start to part. Depending on the deformability of the dispersed $\left(w_{1} / o\right.$ ) droplets, the gap size between the two surfaces would be high for non-deformable droplets, or lower for deformable droplets. For higher deformability, the droplets may form a viscous film or patches of oil film. In this case, the droplets will become effective at reducing the friction coefficient [13]. This might explain the reduction in friction at high entrainment speeds.

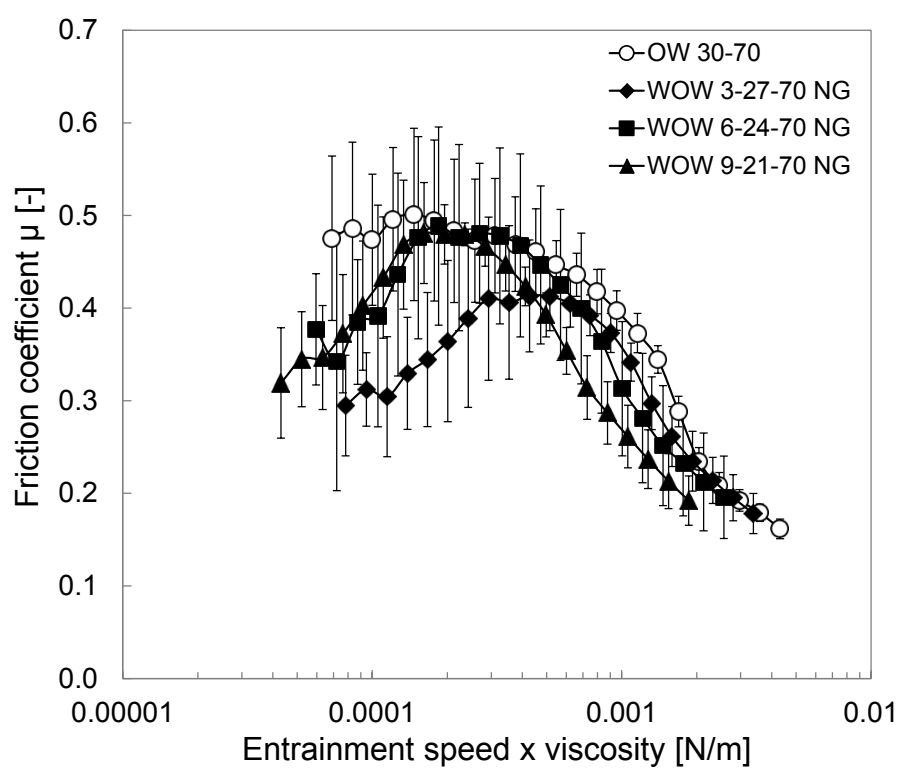

Figure 4.2 Friction coefficients of double emulsions with different fractions of non-gelled inner water phase $w_{1}$ versus entrainment speed $x$ viscosity. The friction coefficients of the corresponding full-fat single $\left(0 / w_{2}\right)$ emulsion are shown with open symbols. Error bars indicate standard deviations.

The increase in friction coefficient in the intermediate entrainment region has also been observed in other studies of emulsion-based foods [9, 14]. Baier and co-workers [14] explained this increase in friction by an exclusion of the sample (low fat milk) from the contact zones. As a consequence, the sample cannot act as a lubricant. They observed this increase in friction particularly in samples with a very low fat 
content $(<2 \%)$, while at higher fat contents, this increase was not found or was less pronounced. In the present study, the amount of dispersed oil phase was always $30 \%$ and therefore contradicts the hypothesis that the increased friction and presence of an inflection point is related to insufficient lubrication due to limited oil entering the gap. The high amount of dispersed phase in our study should be sufficient to provide lubrication. However, the increase in friction may not be related to the amount of the droplets, but the size of the oil droplets. In our study, the oil droplets had diameters $\left(\mathrm{d}_{0.5}\right)$ of about $50 \mu \mathrm{m}$, and in combination with double emulsion droplets being less deformable than pure oil droplets, droplets at first might not have been able to enter the gap at low entrainment speeds since the gap in the boundary regime is expected to be $1 \mu \mathrm{m}$ or smaller [15]. Instead, the continuous water phase $w_{2^{\prime}}$ known to be a poor lubricant, might have entered the gap, thereby increasing the friction coefficient. A similar explanation was postulated by Selway and Stokes [15] who interpreted an increase in friction coefficient of a low-fat thickened cream as an indication that oil was not fully entrained in the contact zone and hence lubrication was dominated by the aqueous phase. An alternative hypothesis is that the oil droplets did enter the gap, but based on their low deformability at low speeds, friction increased since only a small part of their surfaces was in contact with the tribo-surfaces.

The single $\left(\mathrm{o} / \mathrm{w}_{2}\right)$ emulsions displayed higher friction coefficients than all double emulsions at low entrainment speeds. At higher entrainment speeds, double $\left(w_{1} / o / w_{2}\right)$ emulsions and $\left(o / w_{2}\right)$ single emulsions had similar friction coefficients, even though no maximum was found for the single emulsion. At low entrainment speeds, friction is dominated by physical contact of the surfaces, and this regime is known as the boundary regime. In this regime, oil droplets cannot enter the gap and therefore cannot influence friction. At low entrainment speeds, double emulsions had the ability to decrease the friction coefficient in the boundary regime in contrast to single $\left(\mathrm{o} / \mathrm{w}_{2}\right)$ emulsions. In comparison to the single $\left(\mathrm{o} / \mathrm{w}_{2}\right)$ emulsion, double emulsions must contain ingredients other than oil droplets that lead to a decreased friction in the boundary regime.

To explain this effect, we have to consider that water droplets inside the oil droplets have to be stabilized by a lipophilic surfactant. The lipophilic surfactant used in the present study is polyglycerol polyricinoleate (PGPR). An excess of PGPR was used to create small water droplets. PGPR can thus also be present in the continuous oil phase or as micelles in the outer water phase. As PGPR molecules are small and hydrophobic, they can easily enter the gap, adsorb on the hydrophobic PDMS surfaces and provide lubrication. To investigate the effect of PGPR present in the oil or continuous phase 
on lubrication, a series of single $\left(0 / w_{2}\right)$ emulsions differing in PGPR concentrations ( 0 - $4.5 \%$ based on oil phase) were prepared (Table 4.1 and Figure 4.3). Since PGPR lowers the $\left(\mathrm{o} / \mathrm{w}_{2}\right)$ interfacial tension [16], mixing speeds during emulsion preparation were adapted, so that oil droplet sizes of all $\left(\mathrm{o} / \mathrm{w}_{2}\right)$ emulsions were similar $(50 \mu \mathrm{m})$. In this way, we investigate solely the effect of PGPR addition on lubrication.

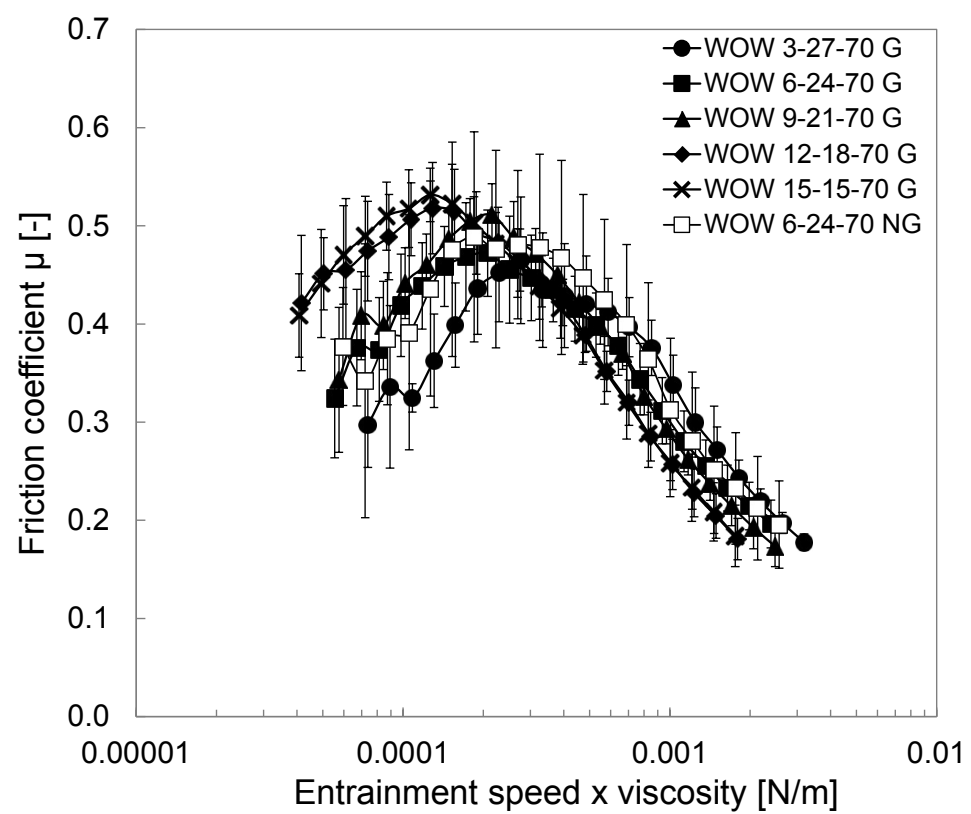

Figure 4.3 Friction coefficients of single $\left(0 / w_{2}\right)$ emulsions containing $30 \mathrm{wt} \%$ dispersed oil phase and between 0 to 4.5 wt\% PGPR (based on oil phase) as a function of entrainment speed $x$ viscosity. Error bars indicate standard deviations.

As can be seen in Figure 4.3, the addition of PGPR tended to decrease the friction coefficient of the single $\left(\mathrm{o} / \mathrm{w}_{2}\right)$ emulsion up to a concentration of $0.2 \mathrm{wt} \%$ at low entrainment speeds. When 0.5 wt\% PGPR or more was present, the friction coefficient at low entrainment speeds was decreased, and the friction coefficient was not further reduced upon an increase in lipophilic emulsifier concentration at $1 \mathrm{wt} \%$ and more. These results indicate that a critical emulsifier concentration was exceeded to decrease friction, since a sufficient concentration of emulsifier needs to be adsorbed at the PDMS surfaces to form an initial lubricating film. Our results contradict those of Douaire and co-workers [17], who did not find a decrease in friction with increasing PGPR concentration for oil-continuous $\left(w_{1} / 0\right)$ emulsions. This can be explained by the fact that their concentrations were much higher (1-7 wt\%), indicating an excess of PGPR. The increase in lubrication found in the present study can be related to adhesion of emulsifier molecules to the tribological surfaces, which has also been observed by 
others $[2,13]$. Liu and co-workers [13] and Bellamy and co-workers [2] showed that the type of emulsifier (WPI or Tween 20 for Liu and co-workers, and Na-caseinate or sucrose ester for Bellamy and co-workers) influenced lubrication properties, and that different emulsifiers interact differently with the surfaces. Since PDMS pins used in the present study are hydrophobic, the hydrophobic emulsifier PGPR might have adsorbed the pin surfaces through hydrophobic interactions, thereby providing lubrication.

An inflection point of the friction coefficient was found when $0.5 \mathrm{wt} \%$ PGPR or more was added to the oil phase of the single emulsions (Figure 4.3). In the boundary regime at low entrainment speeds, friction coefficients were low, followed by an increase in friction coefficient up to a maximum of about 0.35 . At higher entrainment speeds, the effect of PGPR decreased and friction coefficients decreased to similar values as the other $\left(\mathrm{o} / \mathrm{w}_{2}\right)$ emulsions with lower PGPR concentrations. Cambiella and co-workers [18] also studied the effect of type (nonionic, cationic, anionic) and concentration of hydrophilic emulsifiers on lubricating properties of single $\left(\mathrm{o} / \mathrm{w}_{2}\right)$ emulsions and found that especially at higher emulsifier concentrations, the film thickness between the two surfaces first increased with increasing entrainment speed, followed by a collapse to a very thin film occurring at entrainment speeds around $10 \mathrm{~mm} / \mathrm{s}$. Formation (and collapse) of an emulsifier film could also play a role in the emulsions studied here, since PGPR is very hydrophobic and can potentially adsorb at the hydrophobic PDMS surfaces, thereby reducing friction.

With regard to double $\left(\mathrm{w}_{1} / \mathrm{o} / \mathrm{w}_{2}\right)$ emulsions, it is most likely that the adsorption of PGPR onto the PDMS surface of the tribo-pair caused the decrease in friction coefficient at low entrainment speeds, while the entering of water-containing oil droplets into the gap probably explains the lubrication behavior at higher entrainment speeds.

\subsubsection{Effect of gelation of inner water droplets $w_{1}$ on friction of double emulsions}

The results in the previous section indicated that the amount of inner water droplets in double emulsions affects the lubrication behavior. However, the amount of inner $\mathrm{w}_{1}$ droplets that can be dispersed in oil is limited to $30 \%$. To investigate the effect of higher fractions of inner water phase, gelation of those water droplets was necessary (chapter 2). By gelling the inner water droplets, we were able to obtain inner water fractions of up to $50 \%$. In Figure 4.4, the friction coefficient of double emulsions containing gelled droplets (closed symbols) between 3 and $15 \%$ (corresponding to fat reduction levels between 10 and $50 \%$ ), as well as the friction coefficient of a double emulsion with a non-gelled inner water phase (open symbols), are plotted versus the entrainment speed $\mathrm{x}$ viscosity. 


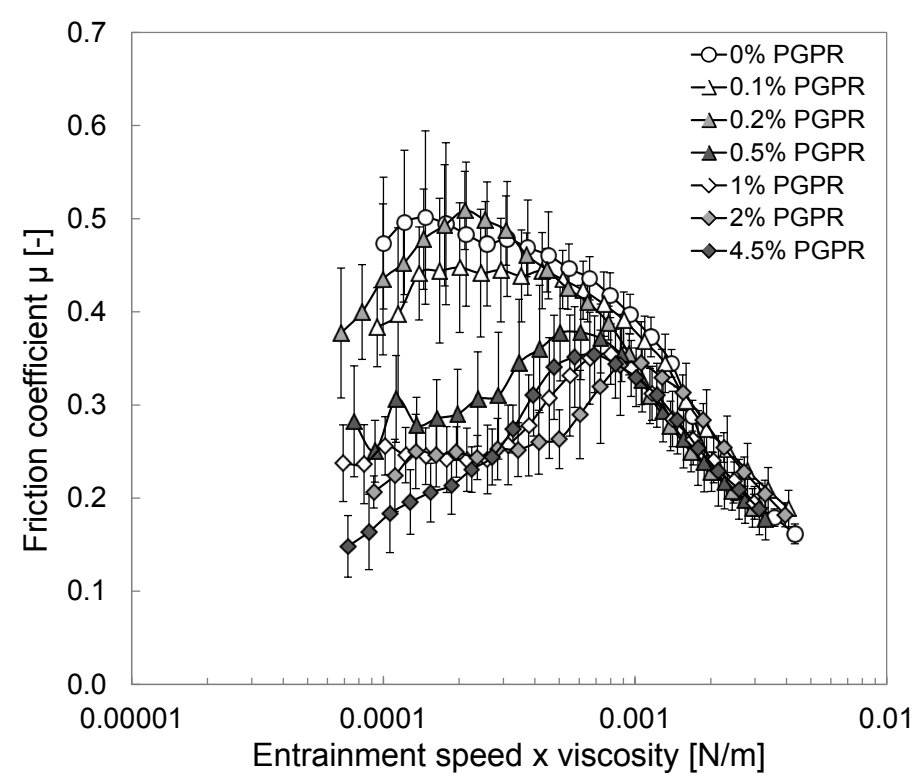

Figure 4.4 Friction coefficients of double emulsions with gelled (closed symbols) and non-gelled (open symbol) inner water phases $w_{1}$ as a function of entrainment speed $x$ viscosity. Double emulsions with a gelled inner water phase contained real fractions of $1.8,6.0,6.3,8.6$, and $11.6 \mathrm{wt} \%$, while the double emulsion with a non-gelled inner water phase contained $3.7 \mathrm{wt} \%$ water. Error bars indicate standard deviations.

Double emulsions with a similar fraction of non-gelled and gelled inner water phase (WOW 6-24-70 NG and WOW 6-24-70 G) showed very similar friction coefficients at all entrainment speeds. We therefore suggest that gelation of water droplets had a minor influence on the lubrication properties and the deformability of the oil droplets at this inner water fraction. In fact, the viscosity of the two corresponding primary $\left(w_{1} / o\right)$ emulsions was nearly identical (Table 4.1).

Even though error bars are large, friction coefficients generally tended to increase upon an increase of the gelled $w_{1}$ droplet fraction. The increase of the gelled $w_{1}$ droplet fraction leads to an increase of viscosity of the dispersed ( $\left.w_{1} / 0\right)$ phase (Table 4.1), particularly when approaching the maximum packing fraction. We hypothesize that this increase in viscosity of the $\left(w_{1} / 0\right)$ emulsion in turn leads to a decrease in deformability of the dispersed $\left(w_{1} / 0\right)$ droplets. Additionally, this increase in viscosity of the dispersed $\left(w_{1} / 0\right)$ phase also leads to an increase in viscosity ratio between the dispersed $\left(w_{1} / 0\right)$ and continuous $\left(w_{2}\right)$ phase. The viscosity ratio also influences the deformability of the droplets. The smaller the viscosity differences between the oil droplets and the continuous phase, the more easily the oil droplets elongate, and the faster they are broken up [19-21]. When viscosity differences between dispersed and continuous phase are large, i.e. the oil droplets being less deformable, oil droplets 
elongate less, and less film formation occurs. This results in increased friction. De Vicente and co-workers [22] indeed showed that the lubrication behavior of $\left(\mathrm{o} / \mathrm{w}_{2}\right)$ emulsions depended on the deformability of the dispersed phase. They showed that less deformable oil droplets could enter the gap between the tribo-surfaces. When large oil droplets did not contain inner water droplets, the droplet viscosity was low and the droplets were thus very deformable. In this case, the droplets may have formed a thin film at the inlet between the two surfaces, and then entered the gap. With increasing inner water phase fraction, the viscosity of the oil droplets increased and deformability decreased, which might have resulted in two effects. Either the decreased deformability increasingly obstructed the oil droplets from entering the contact zone between the two surfaces, or the less deformed oil droplets entered the gap, thereby increasing the film thickness, resulting in a higher friction coefficient. In the case that the droplets would not have entered the gap, the friction coefficient would have remained constant, and therefore this scenario is not likely to have happened in our systems.

Another possible reason for the increase in friction coefficient with increasing concentrations of gelled water droplets in the oil droplets might have been related to the amount of gelled water droplets that are lost during emulsion preparation. In the emulsions studied here, between 0 to $40 \%$ of the water droplets were expelled to the outer water phase, which resulted in fractions between 0.1 and $4.6 \mathrm{wt} \%$ of gelled particles in the outer water phase $\mathrm{w}_{2}$. Inside the oil droplets, those gelled droplets had an average size of $0.2 \mu \mathrm{m}$. When expelled to the outer phase, we expect our gelled droplets in the outer water phase to be of similar size, although they may have slightly swollen based on osmotic pressure differences. To investigate whether the presence of small gelled particles has an effect on the lubrication behavior and can explain some of the results observed, we prepared single $\left(\mathrm{o} / \mathrm{w}_{2}\right)$ emulsions and added a known fraction of gelled particles to the outer water phase. In this way, we investigate the influence of gelled particles in the continuous water phase solely.

Since all single $\left(\mathrm{o} / \mathrm{w}_{2}\right)$ emulsions contained the same amount of dispersed oil phase with similar oil droplet sizes, differences in friction coefficient as depicted in Figure 4.5 were related to gelled $w_{1}$ beads present in the outer water phase $w_{2}$. When gelled particles were added to the outer water phase, friction coefficients were lower at low entrainment speeds, but exceeded those of the single $\left(\mathrm{o} / \mathrm{w}_{2}\right)$ emulsion without gelled particles at increasing entrainment speeds. The initial decrease in friction coefficient was probably linked to remaining PGPR that was introduced in the emulsion system with the gelled particles. It is possible that some PGPR around the gelled particles 
was not completely removed by the washing step during preparation of the gelled particles. Another possibility is that gelled particles entered the gap already at very low entrainment speeds and acted as ball-bearing lubricants [13], as the gelled particles were comparable in size and shape to the microparticulated whey protein used by Liu and co-workers. At higher entrainment speeds, the friction coefficients of those single $\left(\mathrm{o} / \mathrm{w}_{2}\right)$ emulsions containing gelled particles in $\mathrm{w}_{2}$ exceeded those of emulsions without gelled particles. At these higher entrainments speeds, we assume that oil droplets were present in the gap, and that the presence of gelled particles increased the friction even further. However, for an increase in the concentration of the gelled particles, no increase in friction was found.

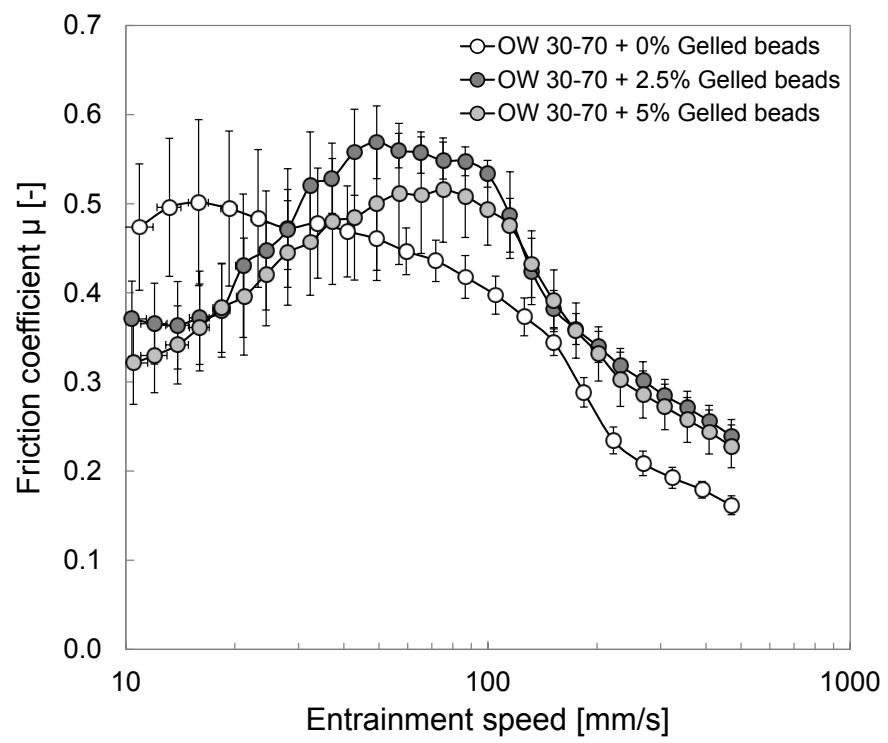

Figure 4.5 Friction coefficients of single $\left(0 / w_{2}\right)$ emulsions with $30 \mathrm{wt} \%$ dispersed oil phase containing $\mathbf{0 , 2 . 5}$ or $\mathbf{5} \mathrm{wt} \%$ of gelled particles in the continuous water phase, as a function of entrainment speed. The percentage of beads represents the total weight of the pellet used. As the maximum packing fraction of the beads is approximately $65 \%$, the amount of beads is slightly lower. Error bars indicate standard deviations.

With regard to the increase in friction coefficient observed in double emulsions at intermediate entrainment speeds (Figure 4.4), three explanations are possible based on the observations in Figure 4.5. First, as shown in Figure 4.5, gelled particles in $\mathrm{w}_{2}$ increase the friction coefficient at intermediate entrainment speeds. Since double emulsions with a larger fraction of gelled $w_{1}$ beads also had higher fractions of expelled gelled $w_{1}$ beads, the effect could be attributed to the presence of the gelled particles. In this case, we would expect a further increase in friction with increasing concentration of gelled particles in the outer water phase (Figure 4.5). However, since we did not observe this, the increased friction cannot be attributed to the gelled 
particles in $w_{2}$ only. Also other factors like the deformability of the oil droplets have to be considered. When the viscosity of the dispersed $\left(w_{1} / 0\right)$ phase is increased due to the addition of small water droplets, deformability of the oil droplets is reduced. As a second effect, we propose that this reduced deformability might lead to oil droplets entering the gap in a less deformed state, thereby suddenly increasing the film thickness and therewith increase the friction. With increasing amounts of (gelled) water droplets, deformability of the oil droplets is further reduced. Additionally, it is possible that gelled particles in the outer water phase reduce the deformability of oil droplets further through obstruction by surrounding gelled particles close to the surfaces at intermediate entrainment speeds. Third, an increase in friction might be related to a collapse of a previously formed emulsifier film of PGPR at the hydrophobic PDMS surface. Above a critical speed, this emulsifier film might collapse, leading to a decrease in film thickness, resulting in an increase in friction.

To summarize, it seems that the lubrication properties of double emulsions with a gelled inner water phase are influenced by a combination of gelled particles in the outer water phase and the deformability of the oil droplets at intermediate entrainment speeds.

\subsubsection{Influence of deformation during tribological measurements on composition of double emulsions}

During the consumption of foods, foods are compressed between the tongue and the palate in the mouth. The gap between the tongue and palate depends on the forces exerted, which have been suggested to be comparable to forces exerted during tribology measurements $[23,24]$. The compression and shear forces during consumption can change the composition of foods, for example by releasing serum or oil upon compression [25]. For double emulsions, we hypothesize that inner water droplets might be released from the oil phase or oil droplets could coalesce when shear forces are applied during a tribological measurement. The loss of inner water droplets may result in changes in oil droplet size and may depend on the fraction of inner water droplets. To test the hypothesis, we investigated the oil droplet size and the change in fraction of (gelled) water droplets remaining in the oil phase before and after a tribological experiment (Table 4.2). After emulsion preparation, double emulsions were sheared in the tribometer at speeds from $0.47 \mathrm{~mm} / \mathrm{s}$ to $470 \mathrm{~mm} / \mathrm{s}$ in $4.3 \mathrm{~min}$, followed by a decrease from $470 \mathrm{~mm} / \mathrm{s}$ to $0.47 \mathrm{~mm} / \mathrm{s}$ in $4.3 \mathrm{~min}$. Samples were removed from the tribometer afterwards and their oil droplet size and yield measured. Real inner water phase fractions were calculated based on the yield as described in section 4.2.3.4. 
Table 4.2 Overview of oil droplet sizes and fractions of gelled water droplets before and after tribological measurements.

\begin{tabular}{|c|c|c|c|c|c|c|c|}
\hline \multirow[b]{2}{*}{ Emulsion } & \multirow[b]{2}{*}{$\begin{array}{l}w_{1} \text { theo- } \\
\text { retical } \\
\text { (wt\% of } \\
\text { double } \\
\text { emulsion) }\end{array}$} & \multicolumn{3}{|c|}{ Real $w_{1}$ in $w_{1} / o / w_{2}$ [wt\%] } & \multicolumn{3}{|c|}{ Oil droplet size $[\mu \mathrm{m}]$} \\
\hline & & Before & After & $\begin{array}{c}\text { Average } \\
\text { relative } \\
\text { change } \\
{[\%]}\end{array}$ & Before & After & $\begin{array}{c}\text { Average } \\
\text { relative } \\
\text { change } \\
{[\%]}\end{array}$ \\
\hline WOW 3-27-70 G & 10 & $1.8 \pm 0.04$ & $1.4 \pm 0.05$ & $-22 \%$ & $51.5 \pm 1.3$ & $53.3 \pm 1.8$ & $+3 \%$ \\
\hline WOW 6-24-70 G & 20 & $6.0 \pm 0.02$ & $4.8 \pm 0.02$ & $-20 \%$ & $50.8 \pm 1.7$ & $52.5 \pm 1.0$ & $+3 \%$ \\
\hline WOW 9-21-70 G & 30 & $6.3 \pm 0.0$ & $4.8 \pm 0.04$ & $-24 \%$ & $48.6 \pm 1.0$ & $48.9 \pm 1.3$ & $+1 \%$ \\
\hline WOW 12-18-70 G & 40 & $8.6 \pm 0.01$ & $7.3 \pm 0.03$ & $-15 \%$ & $51.9 \pm 0.4$ & $50.4 \pm 0.6$ & $-3 \%$ \\
\hline WOW 15-15-70 G & 50 & $11.6 \pm 0.01$ & $9.8 \pm 0.01$ & $-16 \%$ & $54.3 \pm 0.9$ & $56.3 \pm 0.7$ & $+4 \%$ \\
\hline WOW 3-27-70 NG & 10 & $1.3 \pm 0.04$ & $1.2 \pm 0.02$ & $-8 \%$ & $51.9 \pm 0.5$ & $52.8 \pm 0.2$ & $+2 \%$ \\
\hline WOW 6-24-70 NG & 20 & $3.8 \pm 0.02$ & $3.0 \pm 0.03$ & $-21 \%$ & $51.8 \pm 1.1$ & $52.5 \pm 0.5$ & $+1 \%$ \\
\hline WOW 9-21-70 NG & 30 & $4.2 \pm 0.02$ & $3.0 \pm 0.03$ & $-29 \%$ & $47.7 \pm 1.3$ & $50.2 \pm 3.8$ & $+5 \%$ \\
\hline
\end{tabular}

The size of the oil droplets of the double emulsions did not change considerably before and after the tribological experiment and tended to slightly increase by 1 to $5 \%$, while the fraction of inner water droplets decreased considerably after the tribological experiment by up to $29 \%$. This decrease in $w_{1}$ fraction might have been due to the shear stresses occurring during the tribological measurement between the tribo-pair surfaces. Oil droplets that enter the gap are deformed, so that the $\left(\mathrm{o} / \mathrm{w}_{2}\right)$ interfacial area increases. This brings more water droplets into contact with the $\left(\mathrm{o} / \mathrm{w}_{2}\right)$ interface, increasing the probability of coalescence between the water droplets and the outer water phase and expulsion of the gelled droplets. Lower fractions of water droplets would lead to smaller oil droplet sizes. Additionally, high shear stresses between the two surfaces might actually break up the oil droplets. These two effects would lead to a decrease in the oil droplet size. Since a slight increase in droplet size was observed in our experiments, the results imply that also coalescence between the oil droplets occurred during the tribological measurement. Slow re-arrangements of whey proteins at the interface during the deformation might have increased the probability of oil droplet coalescence. We hypothesize that oil droplets broke up and coalesced during deformation so that overall the oil droplet size increased marginally. Comparison of oil droplet size distributions before and after friction measurements (rather than just comparing the averages) did not provide further insights since neither smaller oil droplets (indicating oil droplet breakup) nor larger oil droplets (indicating oil droplet coalescence) were observed.

These results show that the emulsion composition during deformation in a tribological measurement changed considerably due to the release of inner water droplets while oil droplet sizes only slightly increased. These changes might be formulationdependent, and differ with concentration and types of emulsifiers, droplet sizes, and other characteristics. 


\subsubsection{General discussion}

Several mechanisms may play a role in the lubrication properties of double emulsions. In general, we found that friction was decreased at low entrainment speeds, while friction increased with increasing (gelled) inner water phase fractions at intermediate entrainment speeds. At higher entrainment speeds, friction coefficients decreased for all double emulsions.

Based on these observations, we hypothesize that different aspects of the double emulsions dominate the lubrication properties at different entrainment speeds. The influence of the emulsion composition is discussed below.
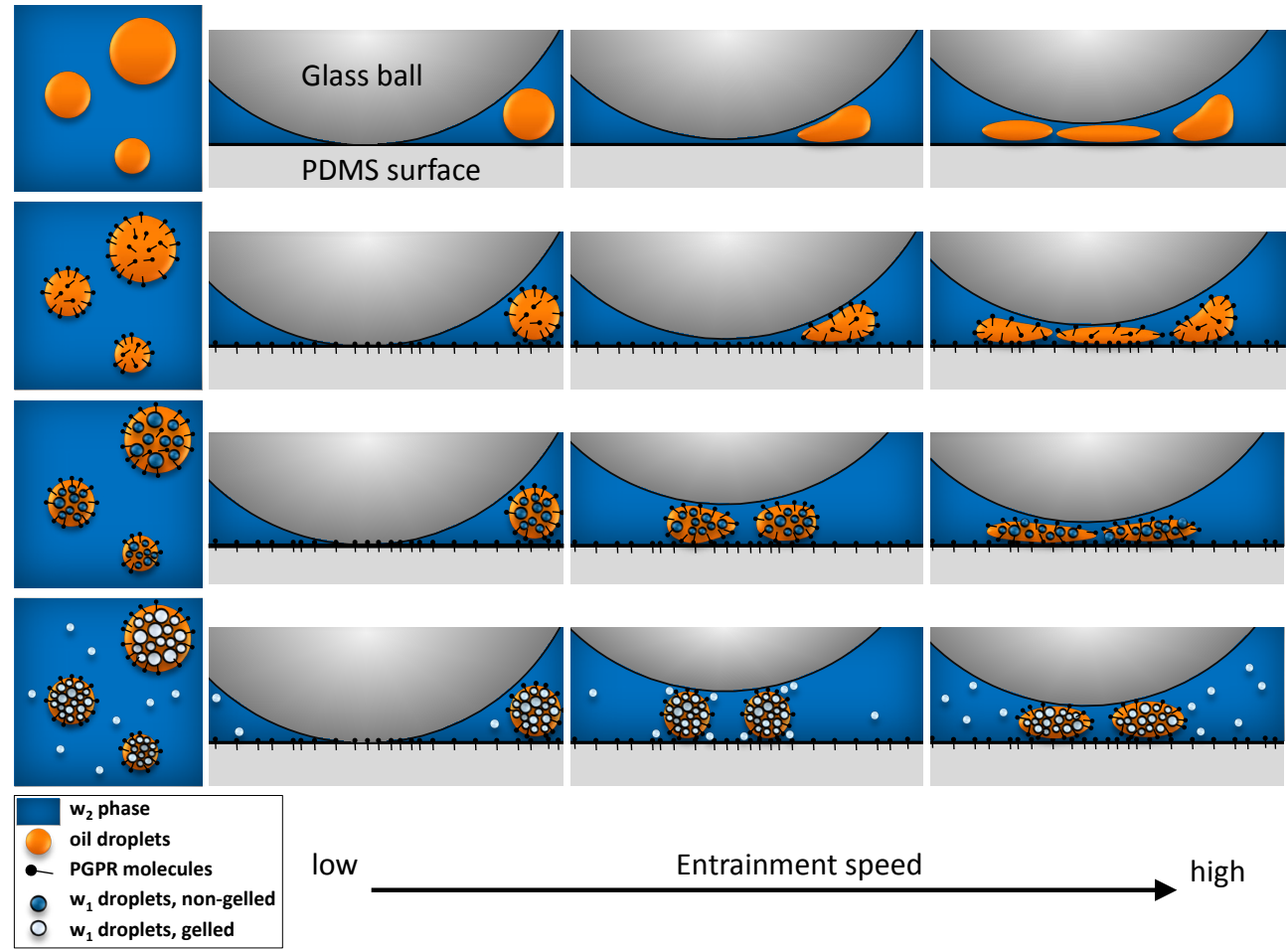

low

Entrainment speed

high

Figure 4.6 Schematic representation of proposed mechanisms contributing to lubrication properties of double emulsions.

As illustrated in Figure 4.6, the addition of PGPR in the emulsion leads to a decrease in friction at low entrainment speeds, probably by an adsorbed emulsifier film at the hydrophobic PDMS surface of the tribo-pair. With increasing entrainment speed, deformable oil droplets enter the gap and lubricate the tribo-surfaces by forming oil film patches. The addition of inner water droplets increases the viscosity of the dispersed $\left(w_{1} / 0\right)$ droplets and thereby reduces the deformability, leading to an increased distance between the surfaces and increased friction at intermediate 
entrainment speeds. With increasing amounts of (gelled) water droplets, deformability of the oil droplets is further reduced. As a consequence, oil droplets provide less lubrication due to less spreading of the oil droplets between the surfaces. This results in increased friction at intermediate entrainment speeds. Gelled particles in the outer water phase increase friction in this regime even further, possibly by further hindering the movement of $\left(w_{1} / o\right)$ droplets. At higher entrainment speeds, the droplets become more deformed, form films and thereby decrease friction.

\subsection{Conclusions}

In the present study, we investigated the influence of composition on lubrication properties of double emulsions. This study focused on the effect of the fraction of inner dispersed aqueous droplets $w_{1}$ and the gelation thereof on the lubrication properties of double emulsions to better understand the mechanisms underlying lubrication properties.

The use of the lipophilic emulsifier PGPR reduced friction at low entrainment speeds probably due to PGPR adsorbing at the hydrophobic PDMS surfaces of the tribo-pair. Increasing fractions of inner water droplets in the oil droplets led to an increase in friction. This is probably due to an increase of the effective viscosity of the dispersed phase, independent of its exact composition. An additional increase in friction was observed in the presence of gelled particles in the outer water phase that hinder the deformation of the oil droplets. Interactions between oil, water, surfactants and the surfaces seem to further influence the lubrication mechanisms. Through these insights into lubrication behavior of double emulsions, advancements in the development of fat-reduced emulsions with similar sensory perception as full-fat emulsions can be achieved. Regarding future research in this field, the effect of temperature and saliva on lubricating properties of double emulsions are of interest to obtain results that are better comparable to in-mouth oral processing of liquids. The effect of temperature on the lubrication behavior of double emulsions with a gelled inner $\mathrm{w}_{1}$ phase using gelatin as the gelling agent is particularly of relevance as gelatin melts at body temperature and may therefore further influence lubrication. This bottomup approach aiming to understand the role of particular components and phases in lubrication processes is important for the development of double emulsions as fat replacers. 


\section{Acknowledgements}

The study received funding from the European Union Seventh Framework Programme (FP7/2007-2013) under grant agreement nr. 289397 (TeRiFiQ project). The authors would like to thank Herman de Beukelaer and Willem Vogelzang for help with the DSC measurements. 


\section{References}

1. van Aken, G.A., M.H. Vingerhoeds, and R.A. de Wijk, Textural perception of liquid emulsions: Role of oil content, oil viscosity and emulsion viscosity. Food Hydrocolloids, 2011. 25: p. 789-796.

2. Bellamy, M., et al., Influence of emulsion composition on lubrication capacity and texture perception. International Journal of Food Science \& Technology, 2009. 44: p. 1939-1949.

3. Le Calvé, B., et al., Fat Perception: How Sensitive are We? Journal of Texture Studies, 2015. 46: p. $200-211$.

4. Joyner, H.S., C.W. Pernell, and C.R. Daubert, Impact of Oil-in-Water Emulsion Composition and Preparation Method on Emulsion Physical Properties and Friction Behaviors. Tribology Letters, 2014. 56: p. 143-160.

5. Malone, M.E., I.A.M. Appelqvist, and I.T. Norton, Oral behaviour of food hydrocolloids and emulsions. Part 1. Lubrication and deposition considerations. Food Hydrocolloids, 2003. 17: p. 763-773.

6. Chojnicka-Paszun, A., H.H.J. de Jongh, and C.G. de Kruif, Sensory perception and lubrication properties of milk: Influence of fat content. International Dairy Journal, 2012. 26: p. 15-22.

7. de Wijk, R.A., J.F. Prinz, and A.M. Janssen, Explaining perceived oral texture of starch-based custard desserts from standard and novel instrumental tests. Food Hydrocolloids, 2006. 20: p. 24-34.

8. Giasson, S., J.N. Israelachvili, and H. Yoshizawa, Thin film morphology and tribology study of mayonnaise. Journal of Food Science, 1997. 62: p. 640-652.

9. Krzeminski, A., et al., Whey protein-pectin complexes as new texturising elements in fat-reduced yoghurt systems. International Dairy Journal, 2014. 36: p. 118-127.

10. de Wijk, R.A. and J.F. Prinz, Mechanisms underlying the role of friction in oral texture. Journal of Texture Studies, 2006. 37: p. 413-427.

11. Liu, K., et al., Fat droplet characteristics affect rheological, tribological and sensory properties of food gels. Food Hydrocolloids, 2015. 44: p. 244-259.

12. de Wijk, R.A. and J.F. Prinz, The role of friction in perceived oral texture. Food Quality and Preference, 2005. 16: p. 121-129.

13. Liu, K., et al., Evidence for ball-bearing mechanism of microparticulated whey protein as fat replacer in liquid and semi-solid multi-component model foods. Food Hydrocolloids, 2016. 52: p. 403-414.

14. Baier, S., et al., A new tribology device for assessing mouthfeel attributes of foods. 5th International Symposium on Food Structure and Rheology, 2009: p. 432-435.

15. Selway, N. and J.R. Stokes, Insights into the dynamics of oral lubrication and mouthfeel using soft tribology: Differentiating semi-fluid foods with similar rheology. Food Research International, 2013. 54: p. 423-431.

16. Gülseren, I. and M. Corredig, Interactions at the interface between hydrophobic and hydrophilic emulsifiers: Polyglycerol polyricinoleate (PGPR) and milk proteins, studied by drop shape tensiometry. Food Hydrocolloids, 2012. 29: p. 193-198.

17. Douaire, M., T. Stephenson, and I.T. Norton, Soft tribology of oil-continuous emulsions. Journal of Food Engineering, 2014. 139: p. 24-30.

18. Cambiella, A., et al., The effect of emulsifier concentration on the lubricating properties of oil-inwater emulsions. Tribology Letters, 2006. 22: p. 53-65.

19. Bentley, B.J. and L.G. Leal, An experimental investigation of drop deformation and breakup in steady, two-dimensional linear flows. Journal of Fluid Mechanics, 1986. 167: p. 241-283.

20. Grace, H.P., Dispersion phenomena in high viscosity immiscible fluid systems and application of static mixers as dispersion devices in such systems. Chemical Engineering Communications, 1982. 14: p. 225-277.

21. Stroeve, P. and P.P. Varanasi, An experimental study on double emulsion drop breakup in uniform shear flow. Journal of Colloid and Interface Science, 1984. 99: p. 360-373.

22. de Vicente, J., H.A. Spikes, and J.R. Stokes, Viscosity Ratio Effect in the Emulsion Lubrication of Soft EHL Contact. Journal of Tribology, 2006. 128: p. 795-800.

23. Stokes, J.R., M.W. Boehm, and S.K. Baier, Oral processing, texture and mouthfeel: From rheology to tribology and beyond. Current Opinion in Colloid \& Interface Science, 2013. 18: p. 349-359.

24. Chen, J. and J.R. Stokes, Rheology and tribology: Two distinctive regimes of food texture sensation. Trends in Food Science and Technology, 2012. 25: p. 4-12.

25. Sala, G., M. Stieger, and F. van de Velde, Serum release boosts sweetness intensity in gels. Food Hydrocolloids, 2010. 24: p. 494-501. 



\section{-

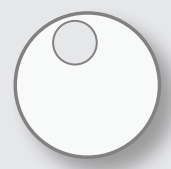

\section{Chapter 5}

\section{Descriptive sensory profiling of double emulsions with gelled and non-gelled inner water phase}
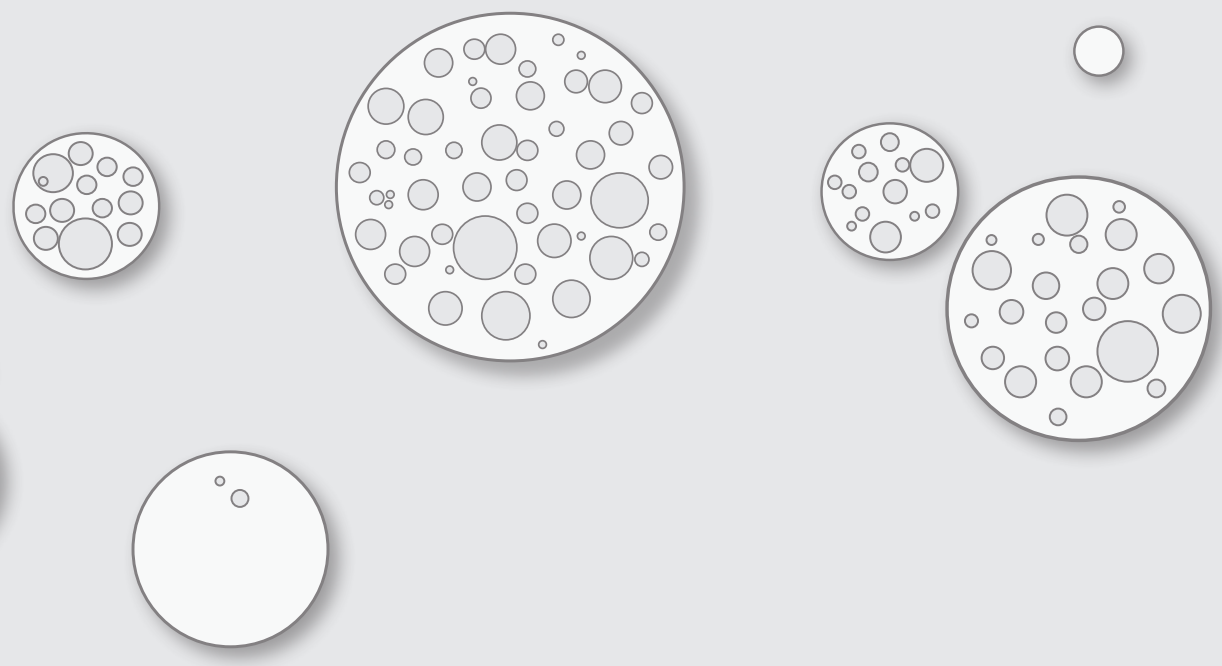

This chapter has been published as

AKL Oppermann, B Piqueras-Fiszman, C de Graaf, E Scholten, M Stieger, Descriptive sensory profiling of double emulsions with gelled and non-gelled inner water phase; Food Research International (2016), 85, 215-223 


\begin{abstract}
The use of double $\left(w_{1} / o / w_{2}\right)$ emulsions has been acknowledged as a promising strategy to reduce oil content in several food applications. Despite the potential of double emulsions for oil reduction, their sensory properties have not been investigated. In this study, we investigated sensory perception of double emulsions by descriptive sensory profiling using a trained panel $(n=11)$. Two sets of emulsions with either 30 or $50 \%$ dispersed phase fraction were studied. Each set differed in composition (gelled and non-gelled inner $w_{1}$ phase, gelatin as gelling agent) and fat reduction level (30 to $50 \%$ ), but was similar in oil droplet size and viscosity. Fat reduction level depended on the amount of water droplets entrapped inside the oil droplets. Emulsions were evaluated on nine attributes describing taste (T), mouth-feel (MF) and after-feel (AF) perception, including thickness (MF), creaminess (MF, AF), fattiness $(M F, A F)$, and cohesiveness (MF). The replacement of oil by small water droplets $w_{1}$ did not decrease the intensity of fat-related attributes. When inner $w_{1}$ droplets were gelled, $47 \mathrm{wt} \%$ of oil could be replaced while increasing the intensity of fat-related attributes. This indicates that the sensory perception of single and double emulsions with gelled and non-gelled $w_{1}$ phase is mainly determined by the total oil droplet surface area. The composition of the inner water phase (gelled or not) also influences the sensory perception of double emulsions. We conclude that fat reduction up to $47 \mathrm{wt} \%$ can be achieved in double emulsions while maintaining or enhancing fatrelated sensory perception.
\end{abstract}




\subsection{Introduction}

The use of water-in-oil-in-water $\left(w_{1} / o / w_{2}\right)$ emulsions offers a potential approach for oil reduction in foods [1]. Double emulsions consist of an inner aqueous phase $\left(w_{1}\right)$ entrapped as small droplets inside larger oil droplets (o) which are dispersed into another aqueous phase $\left(w_{2}\right)$. It has been previously suggested that a double emulsion should evoke similar sensory perception compared to a single $\left(\mathrm{o} / \mathrm{w}_{2}\right)$ emulsion $[2,3]$ when the oil-water interfacial area is the same for both emulsions. To the best of our knowledge, descriptive sensory profiles including fat-related sensory properties (texture and mouth-feel) of double emulsions have not been reported yet. Only few studies have been reported investigating specific sensory properties of double emulsions, for example focusing on saltiness and sourness perception of double emulsions [4-6].

In contrast to the limited number of studies describing specific sensory properties of double emulsions, many studies have discussed systematically the effect of microstructure and physical-chemical properties of single emulsions on sensory perception. Sensory perception of single $\left(\mathrm{o} / \mathrm{w}_{2}\right)$ emulsions is influenced by many emulsion characteristics such as oil droplet size, interfacial properties, viscosity and dispersed phase volume fraction [7]. Both the volume fraction of the dispersed phase and the viscosity of the continuous phase are highly correlated to fat-related attributes such as perceived thickness, fattiness, and creaminess [8,9]. An increased oil volume fraction also leads to more deposition of oral coatings on the tongue surface and increases fatty after-feel perception [10-12]. To compare sensory properties of different emulsions, it is therefore important to control the microstructure and physical-chemical properties of the emulsions.

The aim of this study was to determine the descriptive sensory properties including fat-related attributes of double $\left(w_{1} / o / w_{2}\right)$ emulsions with gelled and non-gelled inner aqueous phases $\left(w_{1}\right)$. We aim to gain a deeper understanding of the role of the amount and composition of inner aqueous phase on sensory perception of double emulsions. Single $\left(\mathrm{o} / \mathrm{w}_{2}\right)$ emulsions were used as full-fat reference stimuli, containing either approximately 30 or $50 \%$ oil. In particular, we investigated (1) the influence of $w_{1}$ phase on sensory perception of fat-related attributes (introducing $w_{1}$ water droplets into the oil phase of a double $\left(w_{1} / o / w_{2}\right)$ emulsion allows to reduce the amount of oil in comparison to full-fat single (o/ $\left.w_{2}\right)$ emulsions), (2) the effect of gelling the inner $w_{1}$ water droplets on sensory perception in comparison to single emulsions and double emulsions with non-gelled inner $w_{1}$ water droplets, and (3) the effect of fat reduction 
level (between 30 and $50 \%$ ) on sensory perception when oil is replaced by gelled $\mathrm{w}_{1}$ water droplets. To minimize the possible effect of oil droplet size and emulsion viscosity on sensory perception, we aimed to keep these properties similar between different single and double emulsions.

\subsection{Materials and methods}

\subsubsection{Materials}

Whey Protein Isolate (WPI, BiPro JE-099-2-420) was obtained from Davisco Foods International Inc. (Le Sueur, MN). The composition of WPI as stated by the manufacturer was $97.7 \%$ protein, $0.3 \%$ fat, $1.8 \%$ ash (dry weight basis). Corrections were made for the impurity of the WPI powder. Polyglycerol polyricinoleate (PGPR 4175) was kindly provided by Palsgaard (Juelsminde, Denmark). Commercial sunflower oil was purchased from a local retailer (Wageningen, the Netherlands). Gelatin (type A, Type 250 PS 30) from Rousselot (Gent, Belgium) was used. $\mathrm{NaCl}$ was obtained from Sigma-Aldrich (purity $\geq 99.5 \%$, Sigma-Aldrich, Steinheim, Germany). For all aqueous solutions, purified water (Milli-Q, $18.2 \mathrm{M} \Omega \mathrm{cm}$ at $25^{\circ} \mathrm{C}$ ) was used.

\subsubsection{Preparation of emulsions}

\subsubsection{Preparation of solutions}

All sample preparations were made based on weight percentages. In all other sections of the article, unless noted otherwise, we refer to volume percentages.

$\mathrm{NaCl}$ solutions were prepared by dissolving $0.4 \mathrm{wt} \% \mathrm{NaCl}$ in purified water. Gelatin solutions were prepared by dissolving $10 \mathrm{wt} \%$ gelatin and $0.4 \mathrm{wt} \% \mathrm{NaCl}$ in purified water at $60{ }^{\circ} \mathrm{C}$ while stirring. The solution was kept at $60{ }^{\circ} \mathrm{C}$ until the gelatin was completely dissolved. The oil phase was prepared by adding either 4,6 or 9 wt $\%$ PGPR to sunflower oil followed by 30 min stirring at $20^{\circ} \mathrm{C}$ (see Table 5.1). Different PGPR concentrations were necessary to obtain similar water droplet size distributions among the used primary $\left(w_{1} / 0\right)$ emulsions. The outer aqueous phase $w_{2}$ was prepared by slowly adding $1 \mathrm{wt} \% \mathrm{WPI}$ powder and $0.2 \mathrm{wt} \% \mathrm{NaCl}$ to purified water while stirring. Stirring was continued for $2 \mathrm{~h}$ at $20^{\circ} \mathrm{C}$. All solutions were prepared one day prior to sensory analysis and stored at $4{ }^{\circ} \mathrm{C}$ overnight before use. All solutions and emulsions were prepared in a food-safe environment. 
Table 5.1 Emulsion compositions and preparation conditions.

\begin{tabular}{|c|c|c|c|c|c|c|}
\hline Sample & $\begin{array}{c}\text { Emulsion } \\
\text { type }\end{array}$ & $\begin{array}{c}\varphi\left(w_{1}\right) \\
{[w t \%] \text { in }} \\
w_{1} / o\end{array}$ & $\begin{array}{c}\text { Gelatin } \\
{[\mathrm{g} / 100 \mathrm{~g}} \\
\left.\mathrm{w}_{1}\right]\end{array}$ & $\begin{array}{c}\text { PGPR } \\
{[\mathrm{g} / 100 \mathrm{~g}} \\
\text { oil] }\end{array}$ & $\begin{array}{c}\Phi\left(w_{1} / o\right) \text { in } o / w_{2} \\
\text { or } w_{1} / o / w_{2}^{*} \\
{[w t \%]}\end{array}$ & $\begin{array}{c}\text { Ultra Turrax } \\
\text { speed } \\
\text { [rpm] }^{* *}\end{array}$ \\
\hline OW 26-74 & $\mathrm{o} / \mathrm{w}_{2}$ & 0 & 0 & 0 & 26 & 7000 \\
\hline WOW 9-21-70 NG & $\mathrm{W}_{1} / \mathrm{o} / \mathrm{w}_{2}$ & 30 & 0 & 4 & 30 & 10000 \\
\hline WOW 9-21-70 G & $\mathrm{W}_{1} / \mathrm{o} / \mathrm{w}_{2}$ & 30 & 10 & 4 & 30 & 10000 \\
\hline WOW 12-18-70 G & $w_{1} / o / w_{2}$ & 40 & 10 & 6 & 30 & 13000 \\
\hline WOW 15-15-70 G & $\mathrm{W}_{1} / \mathrm{o} / \mathrm{w}_{2}$ & 50 & 10 & 9 & 30 & 16000 \\
\hline OW 47-53 & $\mathrm{o} / \mathrm{w}_{2}$ & 0 & 0 & 0 & 47 & 6000 \\
\hline WOW 15-35-50 NG & $\mathrm{W}_{1} / \mathrm{o} / \mathrm{w}_{2}$ & 30 & 0 & 4 & 50 & 9000 \\
\hline WOW 15-35-50 G & $\mathrm{W}_{1} / \mathrm{o} / \mathrm{w}_{2}$ & 30 & 10 & 4 & 50 & 9000 \\
\hline WOW $20-30-50 \mathrm{G}$ & $w_{1} / o / w_{2}$ & 40 & 10 & 6 & 50 & 13000 \\
\hline WOW $25-25-50 \mathrm{G}$ & $\mathrm{w}_{1} / \mathrm{o} / \mathrm{w}_{2}$ & 50 & 10 & 9 & 50 & 16000 \\
\hline
\end{tabular}

\subsubsection{Preparation of $\left(w_{1} / 0\right)$ emulsions}

Primary water-in-oil $\left(w_{1} / 0\right)$ emulsions were prepared by adding between 30 and $50 \mathrm{wt} \%$ of aqueous phase $\mathrm{w}_{1}$ to the oil phase in a $1000 \mathrm{~mL}$ stainless steel container of a Waring blender 8011 ES (Stamford, CT), and mixing at $22000 \mathrm{rpm}$ for $60 \mathrm{sec}$. For samples with gelatin in the inner aqueous phase, both the oil phase and the gelatin solution were heated to $60^{\circ} \mathrm{C}$ in a water bath prior to mixing. We have found in preliminary experiments that for double emulsions with non-gelled inner water droplets (i.e. $w_{1}$ droplets without gelatin), the higher probability of coalescence of the inner water droplets with the outer water phase during preparation of the double emulsions limits the total amount of water droplets that can be incorporated within the oil droplets. Therefore, for emulsions with non-gelled water droplets, the maximum inner dispersed water phase is $30 \mathrm{wt} \%$. To achieve higher fat reduction levels, gelation of the inner water droplets was therefore necessary. For samples containing 40 or $50 \mathrm{wt} \%$ of gelled inner dispersed phase, a premixing step was added: Either 40 or 50 wt\% of gelatin solution was slowly added to the oil phase while increasing the mixing speed of a high speed blender (Ultra Turrax T25 with the dispersing tool S25-N 18G, IKA, Staufen, Germany) from 3000 rpm to 8000 rpm within $1 \mathrm{~min}$ and mixed at $8000 \mathrm{rpm}$ for $4 \mathrm{~min}$. The primary emulsion was then transferred to the Waring blender and prepared as described above. Samples were subsequently cooled under tap water for at least $15 \mathrm{~min}$.

\subsubsection{Preparation of $\left(0 / w_{2}\right)$ and $\left(w_{1} / o / w_{2}\right)$ emulsions}

Double emulsions were prepared by dispersing either 30 or $50 \mathrm{wt} \%$ of the primary $\left(w_{1} / o\right)$ emulsion into either 70 or $50 w t \%$ of outer $w_{2}$ aqueous phase while increasing the mixing speed of the high speed blender (Ultra Turrax T25 with the dispersing tool 
S25-N 18G, IKA, Staufen, Germany) from $3000 \mathrm{rpm}$ to the required speed within one minute. Mixing speed was adapted for each formulation to obtain similar oil droplet size distributions within each set of emulsions. Mixing was continued for $4 \mathrm{~min}$ at the required speed to obtain similar oil droplet sizes. All emulsions were prepared on the day of sensory evaluation and their oil droplet size and viscosity were analyzed to confirm similar characteristics of the emulsions.

Oil-in-water $\left(\mathrm{o} / \mathrm{w}_{2}\right)$ single emulsions were prepared by dispersing either 26 or $47 \mathrm{wt} \%$ oil (without PGPR) to either 74 or $53 \mathrm{wt} \%$ of outer water phase $\mathrm{w}_{2}$ within one minute in a high-speed blender (Ultra Turrax T25 with the dispersing tool S25-N 18G, IKA, Staufen, Germany), while increasing the speed from $3000 \mathrm{rpm}$ to the required speed, at which mixing was continued for $4 \mathrm{~min}$. Dispersed phase fractions of $\left(\mathrm{o} / \mathrm{w}_{2}\right)$ emulsions were adapted to match those of double emulsions after determining their yield and consequently real dispersed phase fractions.

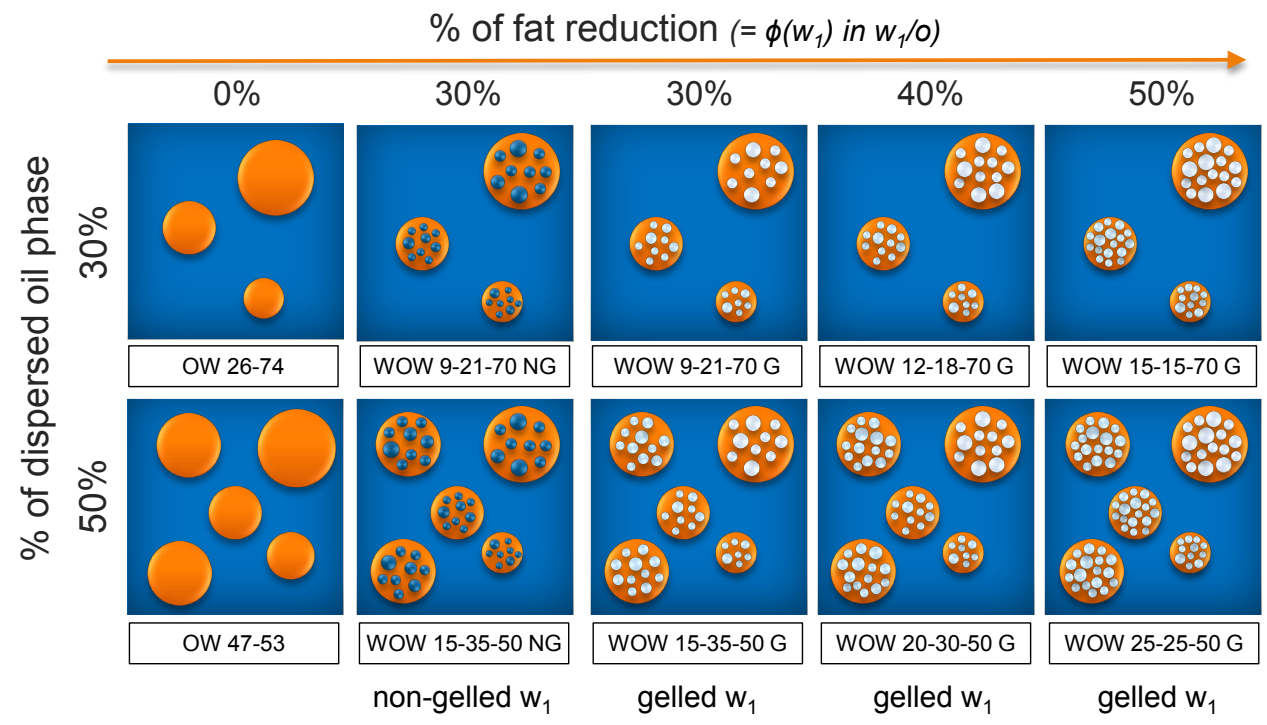

Figure 5.1 Schematic overview of all emulsions.

An overview of compositions and preparation conditions for all single and double emulsions used can be found in Table 5.1. Figure 5.1 provides an illustrative overview of the emulsions. Sample names denote the type of the emulsions ("OW" for single emulsions, "WOW" for double emulsions), the fraction of inner aqueous phase $w_{1}$, oil phase and outer water phase in the final emulsion, followed by the physical state of the inner aqueous phase ("NG" for non-gelled, "G" for gelled). For example, the label "WOW 9-21-70 G" refers to a double emulsion containing $9 \%$ of gelled inner aqueous phase $\mathrm{w}_{1}$ dispersed in $21 \%$ of oil, dispersed in $70 \%$ of outer continuous 
water phase $w_{2}$. The $9 \%$ of gelled inner aqueous phase $w_{1}$ and $21 \%$ of oil correspond to $30 \%$ of dispersed $\left(w_{1} / 0\right)$ emulsion. This corresponds to a fat reduction level of $30 \%$ in the WOW 9-21-70 G emulsion compared to OW 26-74. Single (o/ $\left.\mathrm{w}_{2}\right)$ emulsions do not contain an inner aqueous phase; therefore only the amount of dispersed oil phase (either 26 or $47 \mathrm{wt} \%$ ) and outer water phase (either 74 or $53 \mathrm{wt} \%$ ) are denoted in the sample names.

\subsubsection{Characterization of emulsions}

\subsubsection{Oil droplet size measurements}

Oil droplet sizes of all single $\left(\mathrm{o} / \mathrm{w}_{2}\right)$ and double $\left(\mathrm{w}_{1} / \mathrm{o} / \mathrm{w}_{2}\right)$ emulsions were measured by static light scattering with a Mastersizer 2000 (Malvern Instruments, Worcestershire, UK). The refractive index of the (water-containing) oil droplets was set at 1.45 and the one of demineralized water (continuous phase) at 1.33. Absorption was set at 0.01 . The size distribution of each sample was measured three times and average sizes are reported as $\mathrm{d}_{4,3}$.

\subsubsection{Yield measurements and fat reduction calculations}

DSC (Differential scanning calorimetry) measurements were carried out to determine the inner water fraction within the oil droplets in the double emulsion, defined as yield. The method for determination of yield in double emulsions using DSC and the experimental conditions used have been described previously (chapter 2; [13]).

Based on the yield, we then calculated the real inner dispersed phase fraction (weightbased):

$$
\varphi_{w_{1, \text { real }}}=\text { yield } * \varphi_{w_{1, \text { theoretical }}}
$$

Since the absolute amount of oil in the emulsion system does not change, one can calculate the real dispersed phase fraction $\left(w_{1} / 0\right)$ in the double emulsion (weightbased):

$$
\varphi_{w_{1} / o_{\text {real }}}=\varphi_{w_{1, \text { real }}}+\varphi_{\text {oil }}
$$

Knowing the real inner water fraction and the real dispersed phase fraction $\left(w_{1} / 0\right)$, one can then calculate the real fat reduction (weight-based):

$$
\text { Real fat reduction }=\frac{\varphi_{w_{1, \text { real }}}}{\varphi_{w_{1} / o_{\text {real }}}}
$$




\subsubsection{Rheological measurements}

Viscosity measurements were performed with a stress-controlled Rheometer (MCR 302, Anton Paar). A concentric cylinder (CC17/TI-SN3960, gap width 0.705 mm) was used. Measurements were performed at $20^{\circ} \mathrm{C}$ with a sample volume of $4.7 \mathrm{~mL}$. The viscosity was measured as a function of an increasing shear rate from $0.1 \mathrm{~s}^{-1}$ to $1000 \mathrm{~s}^{-1}$ within $15 \mathrm{~min}$ and 10 data points were collected per decade. Each emulsion was measured once on the day of preparation.

\subsubsection{Sensory analysis}

\subsubsection{Subjects}

The sensory properties of the emulsions were quantified by a sensory panel trained according to the principles of quantitative descriptive analysis $[14,15]$.

The panel consisted of eleven panellists $(n=11)$, of which eight were male, with a mean age of 31.9 years (range from 19 to 54 years). Panellists were recruited and selected based on their sensory ability, basic taste detection, and ability to communicate sensory descriptions of products. Testing took place both in meeting facilities (Agrotechnology and Food Sciences Group, Wageningen University) and in sensory facilities of the Restaurant of the Future (Food and Biobased Research, Wageningen University). An informed consent was signed by all participants at an information session to confirm the awareness of the presence and possible risks of polyglycerol polyricinoleate (PGPR) in some of the products. This study was approved for conduct by the Wageningen University research ethics committee (registered under NL50244.081.14).

\subsubsection{Training}

Eighteen training sessions of 60 minutes each were divided into two stages: attribute generation (four sessions) and training for discriminative ability and reproducibility (fourteen sessions). All sessions were conducted in Dutch by a Dutch-speaking panel leader.

During four training sessions, panellists generated a list with sensory properties to be evaluated by comparing various single $\left(0 / w_{2}\right)$ and double $\left(w_{1} / o / w_{2}\right)$ emulsions with each other. Definitions of the generated attributes were added based on literature $[11,16,17]$. The list of attributes, definitions and instructions for the sensory evaluation was provided to and approved by all panellists. Taste was defined in this study as the gustatory sensations while the product is in the mouth. Mouth-feel 
was referred to the perceived tactile properties during consumption, as defined by Guinard and Mazzucchelli [18], and after-feel was considered to be the mouth-feel after swallowing. Nine attributes were generated in Dutch and translated into English for this manuscript (Table 5.2).

Table 5.2 Overview of sensory attributes and definitions. Attributes are listed in order of sensory evaluation.

\begin{tabular}{lll}
\hline Category & Attribute & Definition \\
\hline Taste (T) & $\begin{array}{l}\text { Saltiness } \\
\text { Sunflower oil taste } \\
\text { Overall taste intensity } \\
\text { Other* }\end{array}$ & $\begin{array}{l}\text { The taste of a salt solution } \\
\text { The taste of sunflower oil } \\
\text { Overall taste intensity }\end{array}$ \\
& $\begin{array}{l}\text { Thickness } \\
\text { Mouth feel (MF) }\end{array}$ & $\begin{array}{l}\text { Resistance to flow before saliva modifies the sample } \\
\text { Amount of fat that is perceived when having the product } \\
\text { in one's mouth for several seconds }\end{array}$ \\
& $\begin{array}{l}\text { Creaminess } \\
\text { Cohesiveness }\end{array}$ & $\begin{array}{l}\text { Soft, velvety and smooth mouth-feel } \\
\text { Compact perception, described as "product remains as a } \\
\text { whole", and "is not easy to swallow" }\end{array}$ \\
& $\begin{array}{l}\text { Fattiness } \\
\text { The fatty layer that remains in the mouth }\end{array}$ \\
& Soft, velvety and smooth perception in the mouth \\
& Other* & \\
\hline
\end{tabular}

*"Other" was included to prevent response restriction or "dumping effect" that occurs when the attribute list lacks a perceived attribute and consequently the panellist would try to report this experience in other attributes, contributing or enhancing the perception of that attribute(s).

Panellists were trained on the evaluation of taste, mouth-feel, and after-feel attributes of the emulsions, and on the use of the $100 \mathrm{~mm}$ continuous line scale anchored at the extremes with "not at all" and "very" (attribute). Panellists were further provided with a 'reference emulsion' ("WOW 15-15-70 G") to facilitate evaluation of perceived intensities of a sample relative to this reference. Average intensities of the nine attributes of the three replicate ratings of the reference emulsion were calculated per panellist and marked on the line scales in individual questionnaires. Throughout the training sessions, panellists were trained on the selected attributes by means of reference products as described in Appendix 5.1. One set of reference products belonged to one specific attribute, e.g. reference products for "saltiness" were $\left(\mathrm{o} / \mathrm{w}_{2}\right)$ emulsions differing in salt concentration. Panellists were asked to rank these reference products using the continuous line scales to train their ability to discriminate similar products with subtle differences in these attributes. In a later stage of the training, panellists also evaluated one of the emulsion sets per training session (either 30 or $50 \%$ dispersed phase).

Training continued until sensory profiles produced by the panellists showed good repeatability, discriminability, and agreement. The performance of the panel was checked with analyses of variance (ANOVA) on the attributes' ratings considering the sample, the panellist (as random effect), the replicate, and all their second order interactions (those with the panellist as random effect) as explanatory variables. 
Results showed a satisfactory panel performance as reflected by the significant effect of the sample, and the non-significant effects of the sample $\times$ panellist and sample $\times$ replicate interactions for the great majority of the attributes [19].

\subsubsection{Sensory profiling evaluations}

Sensory profiling of ten samples (Table 5.1) was carried out in four replicates by $\mathrm{n}=11$ panelists over eight sessions (of 60 minutes each). Each panellist evaluated five samples per session. In odd-numbered sessions they evaluated the samples with 30 vol\% of the primary $\left(w_{1} / 0\right)$ emulsion, and in even-numbered sessions the samples with 50 vol\%. This was done to increase the panellists' focus on differences in sensory characteristics within each set and to limit daily intake of PGPR.

Panellists were asked not to eat or consume any liquids other than water at least $1 \mathrm{~h}$ prior to the start of the test. The emulsions ( $10 \mathrm{~mL}$ per sample) were presented in red-colored $30 \mathrm{~mL}$ medicine cups closed with a lid and labeled with a random 3-digit-code. All samples were offered at room temperature $\left(20^{\circ} \mathrm{C}\right)$. The panel judged each set of model emulsions in a monadic assessment procedure. Presentation order was counterbalanced across panellists to reduce first order and carry over effects (Latin Square Design).

Panellists were seated in sensory booths with white light and slight overpressure and completed the sensory profiling sessions by means of paper questionnaires. Questionnaires were comprised of three categories containing the corresponding attributes with their definitions as well as instructions on how to evaluate the intensity of each attribute on a $100 \mathrm{~mm}$ continuous line scale anchored at the extremes with "not at all" and "very (attribute)", as done during the training sessions. Sensory attributes were assessed in the chronological order as they were perceived. Panellists opened the cup after slightly shaking the sample three times and took a sip of the $10 \mathrm{~mL}$ sample to evaluate taste (T) and mouth-feel (MF) attributes. After swallowing, after-feel (AF) attributes were judged. Panellists indicated the perceived intensity by drawing a vertical line on the continuous line scales. Between each sample evaluation, panellists had a break of 5 min to cleanse their mouths with warm water $\left(40{ }^{\circ} \mathrm{C}\right)$ and white bread without crust. They also used tongue scrapers (budni dent, Hamburg, Germany) to aid the removal of an oral coating after tasting the emulsion samples.

\subsubsection{Statistical data analysis}

To investigate the direct relationship between physical characteristics and sensory perception, within each sample set, a multiple linear regression analysis was conducted on the sensory attributes considering oil droplet size, viscosity, and dispersed phase fraction as explanatory variables. 
A two-way ANOVA (sample as fixed factor and panellist as random factor) was carried out with all ten emulsion samples to study the effect of overall differences of all products, including the dispersed phase fractions (30\% vs. $50 \%$ ), on sensory attributes. Then, each set (30\% or $50 \%$ dispersed phase) was investigated separately to study the differences within each set. Two-way ANOVAs (sample as fixed factor and panellist as random factor) were carried out for all sensory attributes within each set in order to confirm that the samples were significantly different in terms of taste, mouth-feel and after-feel. In case of significant differences, multiple pairwise comparisons were performed with Tukey's Honest Significant Difference (HSD) at $95 \%$ confidence interval. Principal Component Analysis was carried out using the FactoMineR package in $\mathrm{R}$ language [20] on the average ratings of the attributes to identify relations between products and attributes in the perceptual space. Similar ANOVAs were conducted to investigate significant differences between (1) single $\left(\mathrm{o} / \mathrm{w}_{2}\right)$ emulsions vs. double $\left(\mathrm{w}_{1} / \mathrm{o} / \mathrm{w}_{2}\right)$ emulsions with non-gelled $\mathrm{w}_{1}$ droplets, (2) single $\left(\mathrm{o} / \mathrm{w}_{2}\right)$ emulsions vs. double $\left(\mathrm{w}_{1} / \mathrm{o} / \mathrm{w}_{2}\right)$ emulsions with gelled $\mathrm{w}_{1}$ droplets, (3) double $\left(w_{1} / o / w_{2}\right)$ emulsions with non-gelled $w_{1}$ droplets vs. double $\left(w_{1} / o / w_{2}\right)$ emulsions with gelled $w_{1}$ droplets, and (4) among all double $\left(w_{1} / 0 / w_{2}\right)$ emulsions with gelled $w_{1}$ droplets (differing in amount of inner gelled droplets). Data analyses were performed using XIStat (v. 2015.6) and R (v.3.2.1).

\subsection{Results and discussion}

\subsubsection{Emulsion characterization}

\subsubsection{Physical characteristics of emulsions}

An overview of the physical properties of all emulsions can be found in Table 5.3. Emulsions were designed to have similar oil droplet sizes for a given dispersed phase fraction (30 or $50 \%$ ), but different fat reduction levels (equivalent to amount of inner aqueous phase entrapped inside the oil droplets). The inner aqueous phase varied in composition (gelled or non-gelled).

Single and double emulsions with about 30 vol\% dispersed phase had oil droplet sizes $\left(d_{4,3}\right)$ of around $50 \pm 5 \mu \mathrm{m}$, while emulsions with about 50 vol\% dispersed phase fraction had slightly larger oil droplet sizes of around $59 \pm 5 \mu \mathrm{m}$.

Double ( $w_{1} / 0 / w_{2}$ ) emulsions with a non-gelled inner aqueous phase (WOW 9-21-70 NG and WOW 15-35-50 NG) had significantly lower yields than double ( $\left.\mathrm{w}_{1} / \mathrm{o} / \mathrm{w}_{2}\right)$ emulsions with gelled inner aqueous phases. A large fraction of inner water droplets probably 
coalesced with the outer water phase $w_{2}$ during the second emulsification step when $\mathrm{w}_{1}$ was not gelled. This resulted in fat reduction levels of $19 \mathrm{wt} \%$ for WOW 9-21-70 NG and $21 \mathrm{wt} \%$ for WOW 15-35-50 NG instead of the aimed $30 \mathrm{wt} \%$. Higher fat reduction levels with a non-gelled $w_{1}$ phase at a constant oil droplet size could not be achieved, since incorporating more inner dispersed water phase led to more droplet coalescence due to higher packing and concomitant higher collision frequency [21]).

Table 5.3 Physical properties of emulsions. Values of measurements of four batches (one batch per sensory profiling session) were averaged and are listed together with standard deviations.

\begin{tabular}{|c|c|c|c|c|c|c|}
\hline Sample & $\begin{array}{l}\text { Oil droplet } \\
\text { size } d_{4,3}[\mu \mathrm{m}]\end{array}$ & Yield [\%]* & $\begin{array}{c}\Phi\left(\left(w_{1} / o\right) \text { in }\right. \\
\left.\left(w_{1} / o / w_{2}\right)\right) \\
{[v o l \%]}\end{array}$ & $\begin{array}{c}\text { Fat } \\
\text { reduction } \\
\text { [wt\%] }\end{array}$ & $\begin{array}{c}\text { Expelled } \\
\text { gelatin beads } \\
\text { in } w_{2}[w t \%]^{* *}\end{array}$ & $\begin{array}{l}\text { Viscosity } \\
{[\mathrm{mPa} . s]^{* * *}}\end{array}$ \\
\hline OW 26-74 & $52.5 \pm 3.7$ & - & $28.3 \pm 0.0$ & 0 & - & $9.1 \pm 5.3$ \\
\hline WOW 9-21-70 NG & $51.6 \pm 1.7$ & $51.5 \pm 3.9$ & $27.5 \pm 0.4$ & $18.1 \pm 1.1$ & - & $4.4 \pm 2.3$ \\
\hline WOW 9-21-70 G & $49.9 \pm 3.9$ & $84.9 \pm 3.3$ & $30.2 \pm 0.3$ & $26.7 \pm 0.8$ & $1.9 \pm 0.4$ & $6.4 \pm 2.3$ \\
\hline WOW 12-18-70 G & $49.0 \pm 2.7$ & $79.5 \pm 2.9$ & $28.8 \pm 0.3$ & $34.6 \pm 0.8$ & $3.4 \pm 0.5$ & $5.8 \pm 2.7$ \\
\hline WOW 15-15-70 G & $52.9 \pm 1.7$ & $80.5 \pm 4.4$ & $28.0 \pm 0.6$ & $44.6 \pm 1.4$ & $4.0 \pm 0.9$ & $8.9 \pm 3.6$ \\
\hline OW 47-53 & $64.6 \pm 2.5$ & - & $51.1 \pm 0.0$ & 0 & - & $23.7 \pm 3.4$ \\
\hline WOW 15-35-50 NG & $59.6 \pm 2.5$ & $63.5 \pm 1.5$ & $47.6 \pm 0.2$ & $21.4 \pm 0.4$ & - & $10.0 \pm 2.1$ \\
\hline WOW 15-35-50 G & $58.9 \pm 4.1$ & $89.7 \pm 4.4$ & $51.1 \pm 0.6$ & $27.8 \pm 1.0$ & $3.0 \pm 1.2$ & $13.5 \pm 1.5$ \\
\hline WOW $20-30-50 \mathrm{G}$ & $55.5 \pm 0.8$ & $90.7 \pm 2.5$ & $50.2 \pm 0.5$ & $37.7 \pm 0.6$ & $3.6 \pm 0.9$ & $14.2 \pm 0.7$ \\
\hline WOW $25-25-50 \mathrm{G}$ & $58.2 \pm 1.6$ & $88.4 \pm 2.2$ & $48.6 \pm 0.5$ & $46.9 \pm 0.6$ & $5.5 \pm 1.0$ & $17.4 \pm 3.8$ \\
\hline
\end{tabular}

Gelling the inner aqueous phase retains the inner droplets within the oil droplets during the second emulsification step, and resulted in higher yields between 80 and 90 \% for WOW 9-21-70 G, WOW 12-18-70 G, WOW 15-15-70 G, WOW 15-35-50 G, WOW 20-30-50 G, and WOW 25-25-50 G. This gave fat reduction levels between 27 and $47 \mathrm{wt} \%$ depending on the initial amount of incorporated $w_{1}$ phase, close to the aimed values of 30,40 and $50 \mathrm{wt} \%$ fat reduction.

Yields for double emulsions with about $50 \%$ dispersed phase were slightly higher than for double emulsions with about $30 \%$ dispersed phase. This can probably be explained by the slightly larger oil droplet sizes, since it has been shown previously that yields are correlated to oil droplet size [22, 23]. However, for all double emulsions, a fraction (between 9 and $20 \mathrm{wt} \%$ ) of gelled $\mathrm{w}_{1}$ droplets was expelled into the outer water phase $w_{2}$ during the second emulsification step. This means that in the double emulsions, the outer water phase contains between $1.9 \mathrm{wt} \%$ and $5.5 \mathrm{wt} \%$ of gelatin beads (Table 5.3).

The calculated dispersed phase volume fractions varied for emulsions OW 26-74, WOW 9-21-70 NG, WOW 9-21-70 G, WOW 12-18-70 G, and WOW 15-15-70 G between 27.5 and 30.2 vol\% of dispersed phase, and for emulsions OW 47-53, WOW 15-35-50 NG, WOW 15-35-50 G, WOW 20-30-50 G, and WOW 25-25-50 G between 47.6 and 51.1 vol\% of dispersed phase. 
The average viscosities at shear rates between $10 \mathrm{~s}^{-1}$ and $100 \mathrm{~s}^{-1}$ of the emulsions with 30 vol\% dispersed phase ranged from 4 to $9 \mathrm{mPa}$.s. Average viscosities at shear rates between $10 \mathrm{~s}^{-1}$ and $100 \mathrm{~s}^{-1}$ of the emulsions with 50 vol\% dispersed phase ranged from 10 to $23 \mathrm{mPa}$.s. As mentioned before, oil droplet sizes and emulsion viscosity can influence the sensory perception of emulsions $[8,9,11]$. To investigate whether the small differences found in oil droplet size, dispersed phase volume fraction and/ or emulsion viscosity affected sensory perception, correlation analyses were carried out and are discussed in section 5.3.1.2.

To summarize, two series of emulsions differing in dispersed oil phase volume fraction (around 30 vol\% and around 50 vol\%) were obtained. For both dispersed oil phase volume fractions, several emulsions differing in level of fat reduction but with similar oil droplet size, and roughly similar rheological properties were obtained.

\subsubsection{Effect of physical properties of emulsions on sensory perception}

Multiple linear regressions were conducted on each sensory attribute considering oil droplet size, dispersed oil phase fraction, and viscosity as explanatory variables. These analyses were performed separately within each sample set (series of five emulsions with around 30 vol\% and series of five emulsions with around $50 \%$ dispersed oil phase). For the set of emulsions with 30 vol\% dispersed phase, viscosity and dispersed oil phase fraction did not significantly affect any of the sensory attributes, and only oil droplet size was significantly negatively correlated with thickness perception ( $r=-0.576, p=0.008$ ). For the emulsions with 50 vol\% dispersed phase, neither viscosity, dispersed oil phase fraction nor oil droplet size did significantly affect any of the sensory properties, so none of the physical properties was a predictor of sensory variability among the samples (all $R^{2}<0.37$, and all the factors were non-significant). To summarize, the effect of small differences in oil droplet size, viscosity and dispersed oil phase fraction within sample sets had only very minor effects on sensory attributes. Therefore, any differences perceived in the double emulsions are a result of the fat reduction level or the physical characteristics of the inner water droplets.

\subsubsection{Sensory perception of single $\left(0 / w_{2}\right)$ and double $\left(w_{1} / 0 / w_{2}\right)$ emulsions}

\subsubsection{General results}

A two-way ANOVA (samples and panellists) with interaction was applied to all ten samples to sensory scores for the nine attributes evaluated (see Table $\mathbf{5 . 2}$ for attributes). As shown in Figure 5.2, the panel clearly discriminated between the set of emulsions with 30 vol\% dispersed phase and the set of emulsions with 50 vol\% 
dispersed phase. The first two principal components (PC) explained $97.7 \%$ of the total variance, and as expected, the main discrimination dimension (PC1) was based on dispersed oil fraction. Since the main interest of this study was to investigate differences within each set of samples, further statistical analyses on each set of emulsions were separately conducted.

For each set of emulsions, a two-way ANOVA (samples and panellists) with interaction was applied to sensory scores for the nine attributes evaluated. As can be seen in Table 5.4, mean intensities of sensory attributes for emulsions with $50 \mathrm{vol} \%$ dispersed phase are higher for all attributes except saltiness compared to emulsions with 30 vol\% dispersed phase. Significant differences between samples $(p<0.05)$ were found for overall taste intensity, thickness, creaminess (MF and AF), and cohesiveness for the emulsions with about 30 vol\% dispersed phase, and for saltiness, overall taste intensity, thickness, creaminess (MF and AF) and cohesiveness for the emulsions with about 50 vol\% dispersed phase.

Figure 5.3 (PCA for five emulsions with around 30 vol\% dispersed oil phase) and Figure 5.4 (PCA for five emulsions with around 50 vol\% dispersed oil phase) show the results of the two principal component analyses (PCA) within each set of emulsions performed on the sensory attributes. The first two PCs explained $93 \%$ of the total variance for the emulsions with $30 \%$ dispersed phase, and $96 \%$ of the total variance for the emulsions with $50 \%$ dispersed phase. Product plots (Figures 5.3A and 5.4A) show the distribution of emulsions with the same dispersed phase fraction (30 and 50 vol\%, respectively) on the sensory space. The loading plots (Figures 5.3B and 5.4B) reveal the interrelationships among sensory attributes and emulsions. The main discrimination dimension (PC1) was based on oil droplet properties, related to their composition (gelled vs. non-gelled). Relative to the single $\left(\mathrm{o} / \mathrm{w}_{2}\right)$ emulsion and the double $\left(w_{1} / o / w_{2}\right)$ emulsion with a non-gelled inner aqueous phase (on the left side of the plot), all double $\left(w_{1} / o / w_{2}\right)$ emulsions with a gelled inner aqueous phase (on the right side) were more highly related to increasing levels of all perceived attributes. Thus, it seems that the gelled inner aqueous phase strongly contributed to mouth-feel through creaminess, thickness, and cohesiveness, but also enhanced taste intensity. Separate statistical comparisons of the samples within each set will be described in more detail in the next paragraphs. 
Individuals factor map (PCA)

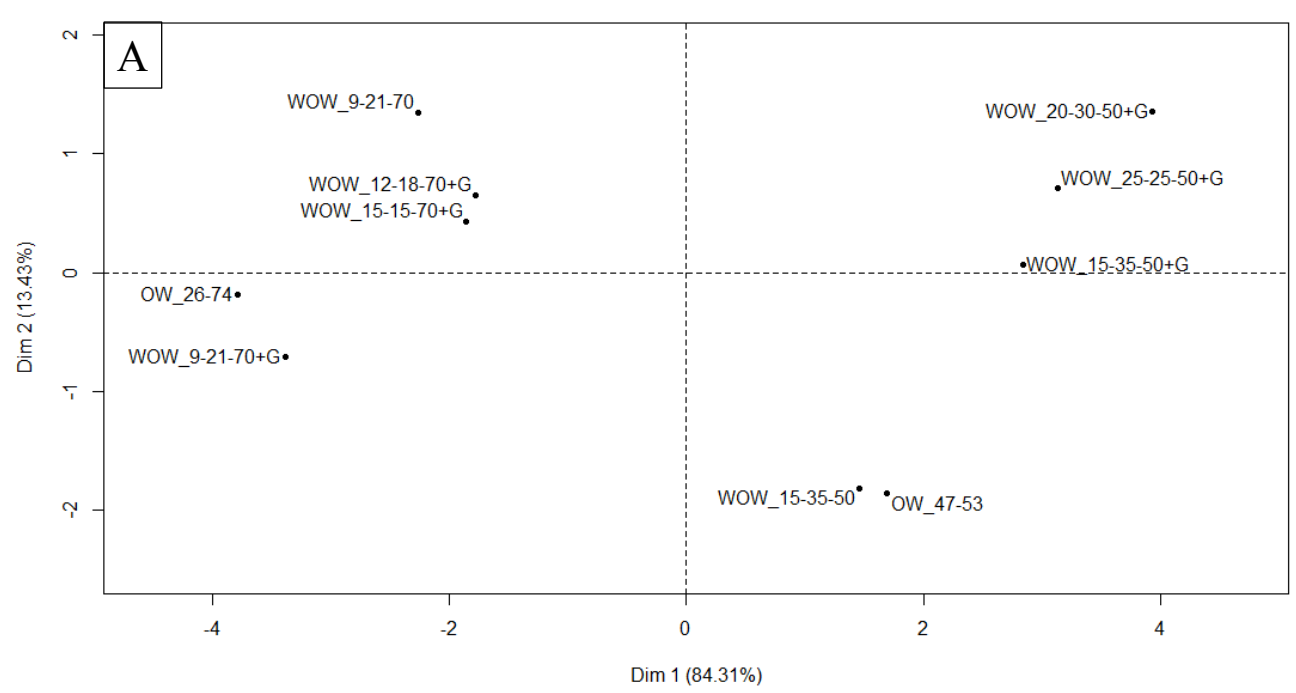

Variables factor map (PCA)

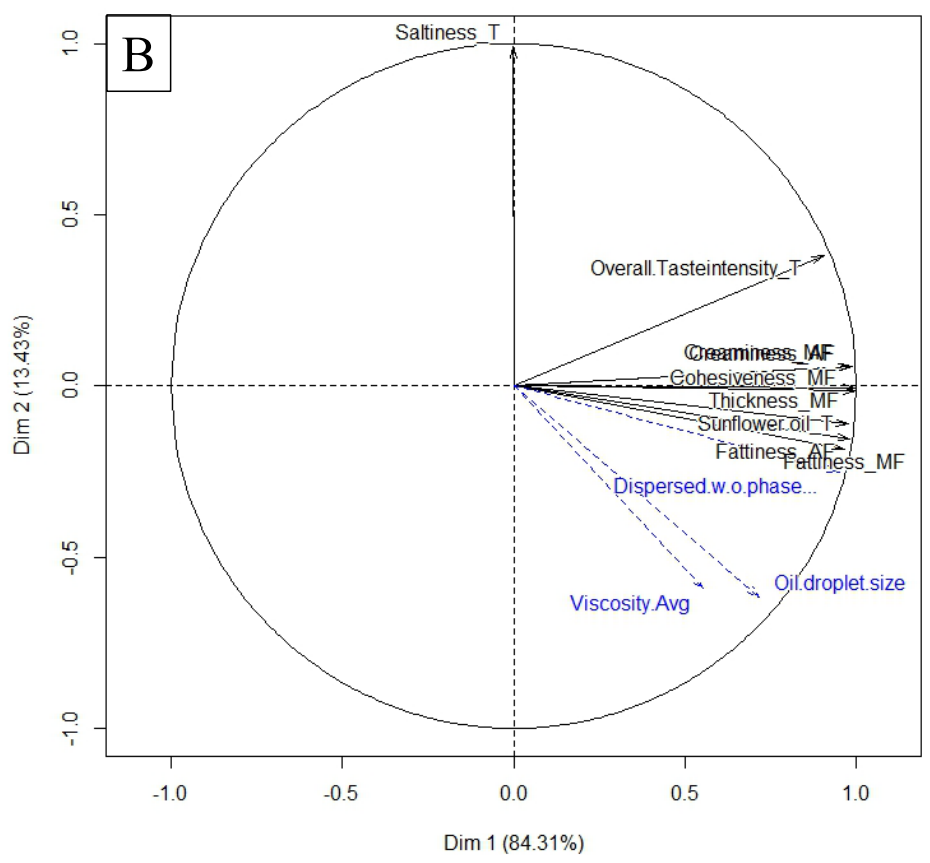

Figure 5.2 Principal Component Analysis (PCA) of all emulsions, (A) product map, (B) loading plot 

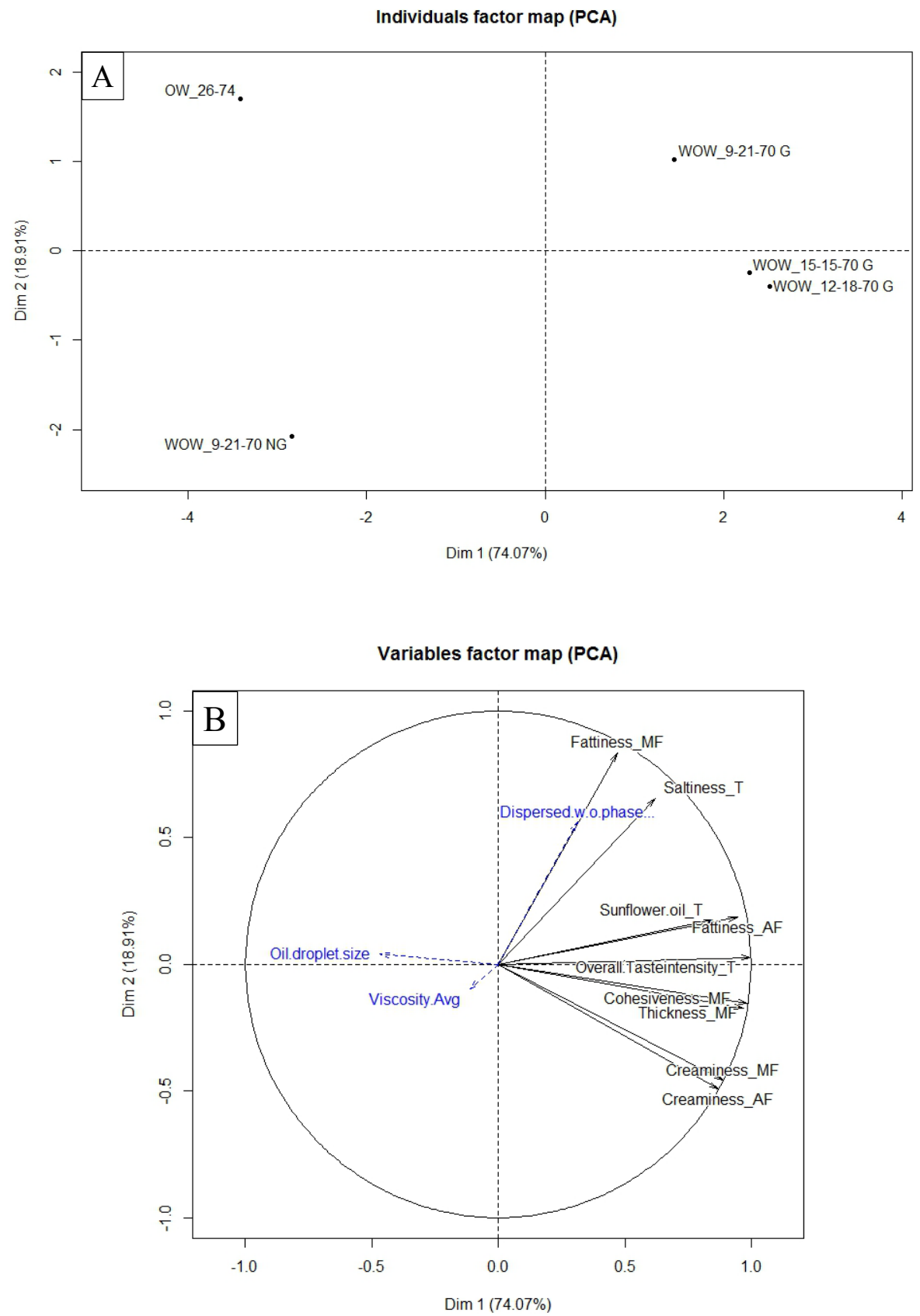

Figure 5.3 Principle Component Analysis (PCA) for emulsions with 30 vol\% dispersed phase, (A) product map, (B) loading plot 
Individuals factor map (PCA)
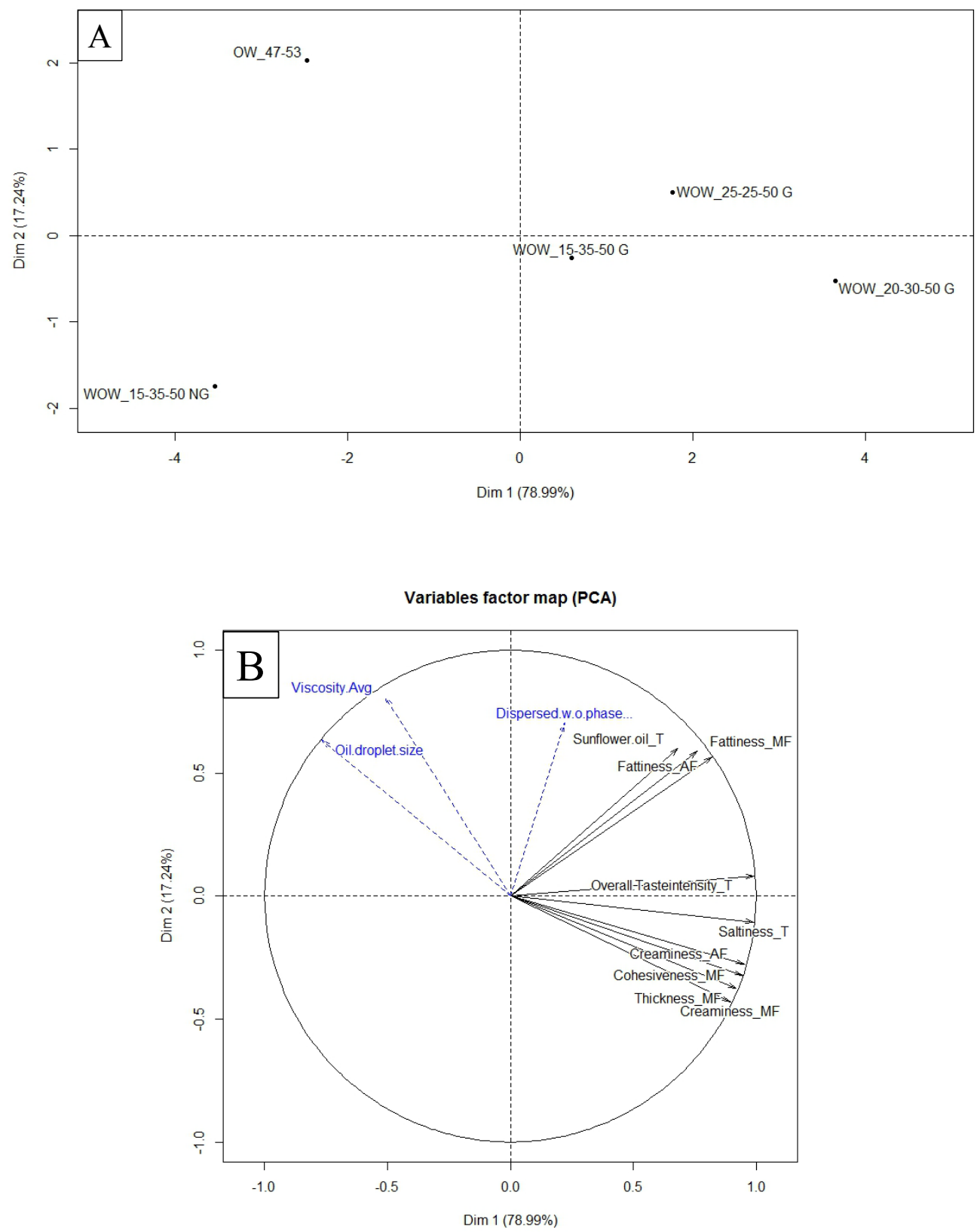

Figure 5.4 Principle Component Analysis (PCA) for emulsions with 50 vol\% dispersed phase, (A) product map, (B) loading plot 


\subsubsection{Effect of introducing an inner aqueous phase on sensory perception: single $\left(0 / w_{2}\right)$ vs. double $\left(w_{1} / 0 / w_{2}\right)$ emulsions with non-gelled $w_{1}$ phase}

To gain insights in multivariate relationships among sensory properties of double $\left(w_{1} / o / w_{2}\right)$ compared to single $\left(o / w_{2}\right)$ emulsions, first the effect of replacing part of the oil with small non-gelled water droplets is discussed.

For the emulsions with 30 vol\% dispersed oil phase, significant differences were observed between OW 26-74 and WOW 9-21-70 NG only for creaminess (MF and AF), for which the sample with the inner water droplets (WOW 9-21-70 NG) was perceived as creamier (MF: $M=3.9$ vs. 3.3, $p=0.013 ; \mathrm{AF}: M=3.6$ vs. 3.1, $p=0.026$ ) (see Figure 5.5A). For the emulsions with 50 vol\% dispersed phase, no significant differences were found between OW 47-53 and WOW 15-35-50 NG for any of the sensory attributes (results not shown). Even though emulsion WOW 9-21-70 NG contained 18 wt\% less oil than emulsion OW 26-74, and oil droplet properties are altered by the inclusion of small water droplets, increasing for example the viscosity of the oil droplets from about $60 \mathrm{mPa}$.s (bulk sunflower oil) to $190 \mathrm{mPa} . \mathrm{s}$ ( $\mathrm{w}_{1} / \mathrm{o}$ emulsion with $30 \mathrm{wt} \%$ nongelled $\left.w_{1}\right)$, inclusion of the inner water droplets did not considerably influence sensory perception. These findings provide further evidence for the suggestion that the perception of fat-related sensory attributes in emulsions is not determined by the bulk properties of the dispersed phase (i.e., presence of an inner aqueous phase $w_{1}$ or different viscosity) but by the total oil droplet surface area. Since the oil droplet size and dispersed phase fraction was similar, the total oil droplet surface area was similar for the different emulsions. Regarding the effect of dispersed phase fraction (30 and $50 \mathrm{vol} \%)$, more differences in sensory perception of fat-related attributes were found in emulsions with 30 vol\% dispersed phase, compared to emulsions with 50 vol\% dispersed phase. For a higher dispersed phase fraction, the overall viscosity increases due to a higher packing of (water-filled) oil droplets. This increase in viscosity may overrule other effects that may be caused by the inclusion of water droplets inside the oil droplets.

\subsubsection{Effect of introducing a gelled inner aqueous phase on sensory perception: single $\left(0 / w_{2}\right)$ vs. double $\left(w_{1} / 0 / w_{2}\right)$ emulsions with gelled $w_{1}$ phase}

As can be seen in Table 5.4, intensities of most attributes increased when the inner aqueous phase was gelled compared to the corresponding single $\left(\mathrm{o} / \mathrm{w}_{2}\right)$ emulsion. For both sets of emulsions (30 and 50 vol\% dispersed phase), all mouth-feel and after-feel attributes, except fattiness (MF and $\mathrm{AF}$ ), were perceived significantly higher for the double emulsion with a gelled $w_{1}$ phase (WOW 9-21-70 G and WOW 15-35-50 G) in comparison to the corresponding single (o/ $\mathrm{W}_{2}$ ) emulsions (OW 26-74 and OW 47-53). 
Figure 5.5B shows the comparison for the emulsion with $30 \%$ dispersed phase and $30 \%$ fat reduction (WOW 9-21-70 G) and the corresponding single emulsion (OW 26-74). Similar results were found for the emulsions with a $50 \%$ dispersed phase: Emulsions OW 47-53 and WOW 15-35-50 G differed also significantly for thickness and creaminess (MF and AF) perception $(p<0.01)$ and for cohesiveness $(p<0.05)$.
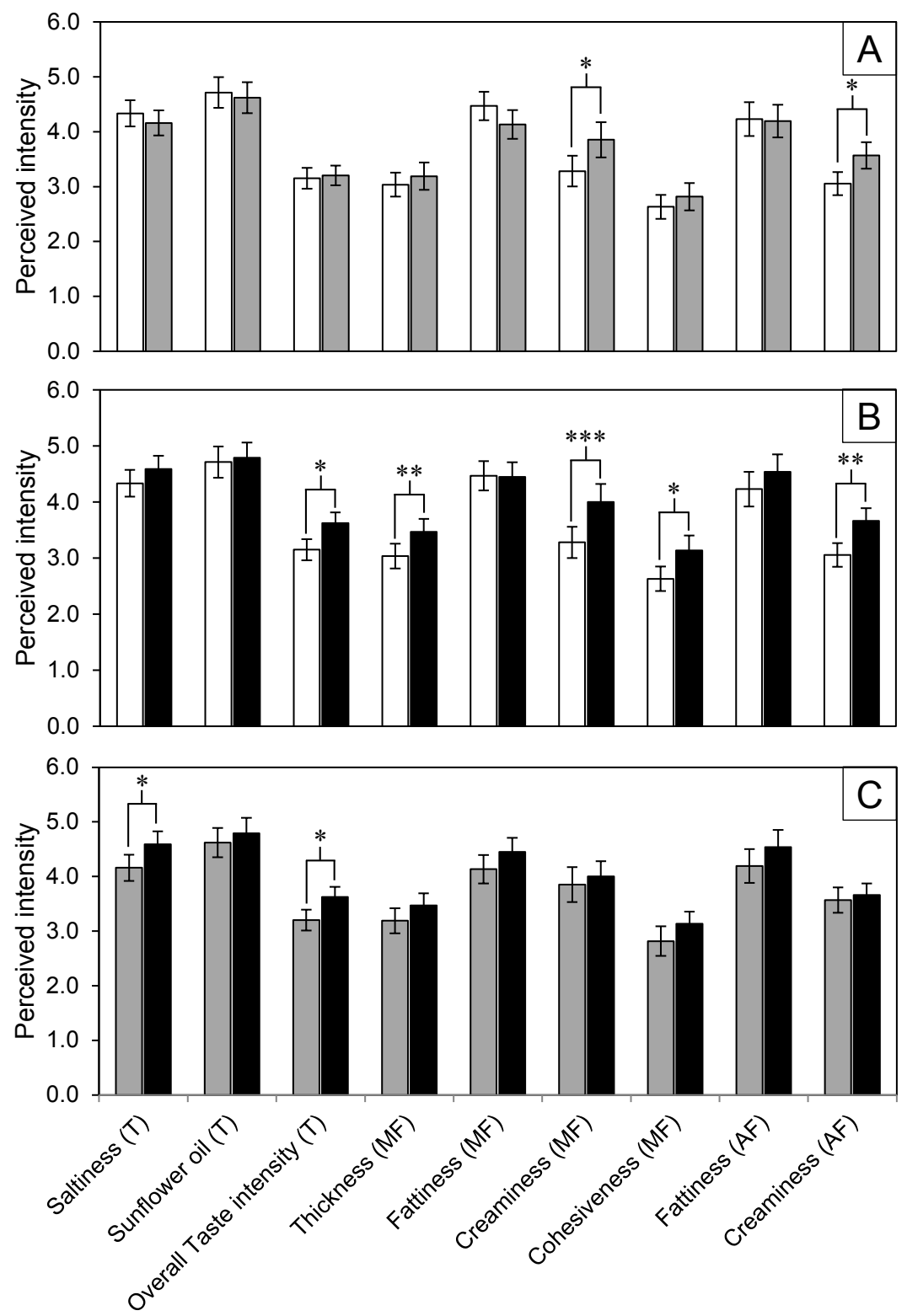

Figure 5.5 Comparison of sensory profiles (mean intensities of all sensory attributes) of specific emulsions: (A) OW 26-74 (white) vs. WOW 9-21-70 NG (grey), (B) OW 26-74 (white) vs. WOW 9-21-70 G (black) and (C) WOW 9-21-70 NG (grey) vs. WOW 9-21-70 G (black). Significant differences indicated with asterisks as * $p$ $<0.05,{ }^{* *} \mathbf{p}<0.01$, and ${ }^{* * *} \mathbf{p}<0.001$. Error bars indicate standard errors. 


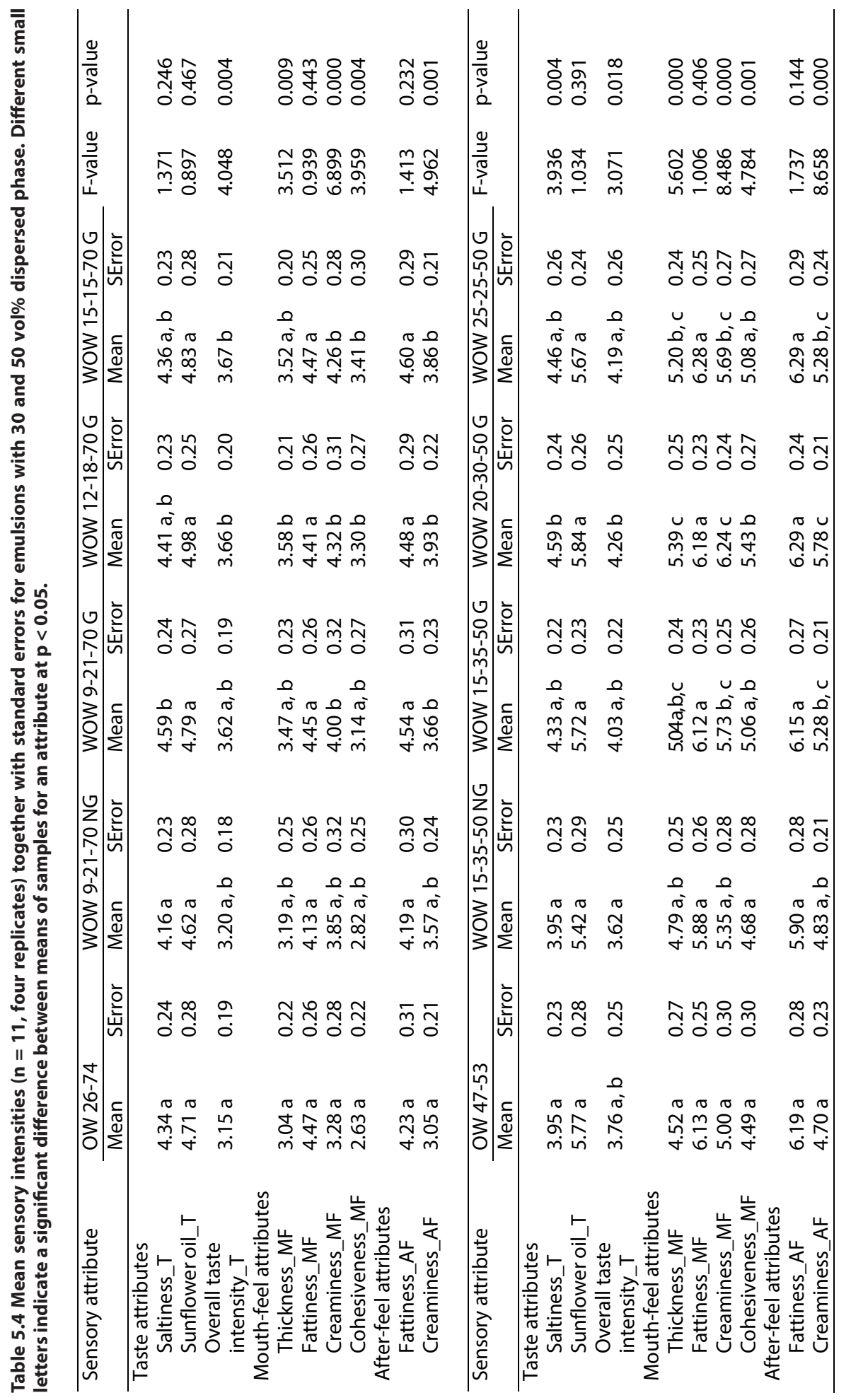


Several mechanisms might underlie the increase in perception of fat-related mouthfeel and after-feel attributes, when gelled water droplets are introduced into the oil droplets. First, the introduction of gelled $w_{1}$ droplets might change the deformability of the oil droplets. Deformability of oil droplets is linked to the oil viscosity. Dresselhuis and co-workers [24] found that oils with a higher viscosity result in lower friction forces and that lower friction forces are correlated with enhanced sensory perception. Friction forces occur within the mouth when the emulsions interact with saliva and are sheared between tongue and palate. Another mechanism could be that during the preparation of double emulsions, some of the gelled beads are expelled from the oil droplets into the outer water phase $w_{2}$. As shown in Table 5.3, the outer water phase $w_{2}$ contained around $1.9 \mathrm{wt} \%$ of gelatin beads for double emulsions with $30 \mathrm{wt} \%$ gelled inner water droplets (WOW 9-21-70 G). For emulsions with a total dispersed fraction of 50 vol\% (WOW 15-35-50 G), the amount of gelatin beads increased to $3 \mathrm{wt} \%$. Melting of gelatin has been shown previously to increase perception of fat-related sensory attributes [25], which may be attributed to the formation of a liquid lubricant layer between the oral surfaces, and thereby reducing friction [26]. These results show that the presence of gelled inner water droplets increased the perception of most fat related attributes. However, it is not clear whether this is an effect of the gelation of the droplets or just an effect of the presence of the gelled droplets.

\subsubsection{Effect of gelling the inner aqueous phase on sensory perception: double $\left(w_{1} / 0 / w_{2}\right)$ emulsions with non-gelled $w_{1}$ phase vs. double $\left(w_{1} / 0 / w_{2}\right)$ emulsions with gelled $w_{1}$ phase}

To gain more understanding whether the additional gelling of the inner aqueous droplets has an effect on the sensory perception, we have also compared double emulsions with a non-gelled aqueous phase to those with a gelled inner aqueous phase.

Figure 5.5C shows the results for emulsions with a total dispersed fraction of $30 \mathrm{vol} \%$ and a fat reduction level of $30 \mathrm{wt} \%$ in the case the droplets are not gelled (WOW 9-21-70 NG) or gelled (WOW 9-21-70 G). The Figure shows that the sample with a gelled $w_{1}$ phase (WOW 9-21-70 G) was perceived more intense with respect to saltiness and overall taste intensity $(p=0.05)$, but all other attributes were not perceived as significantly different. Similar trends were observed for the 50 vol\% double emulsions although these trends were not significant $(p=0.07$ for the two attributes). These results suggest that gelation of the inner water droplets did not significantly affect mouth-feel and after-feel perception. 


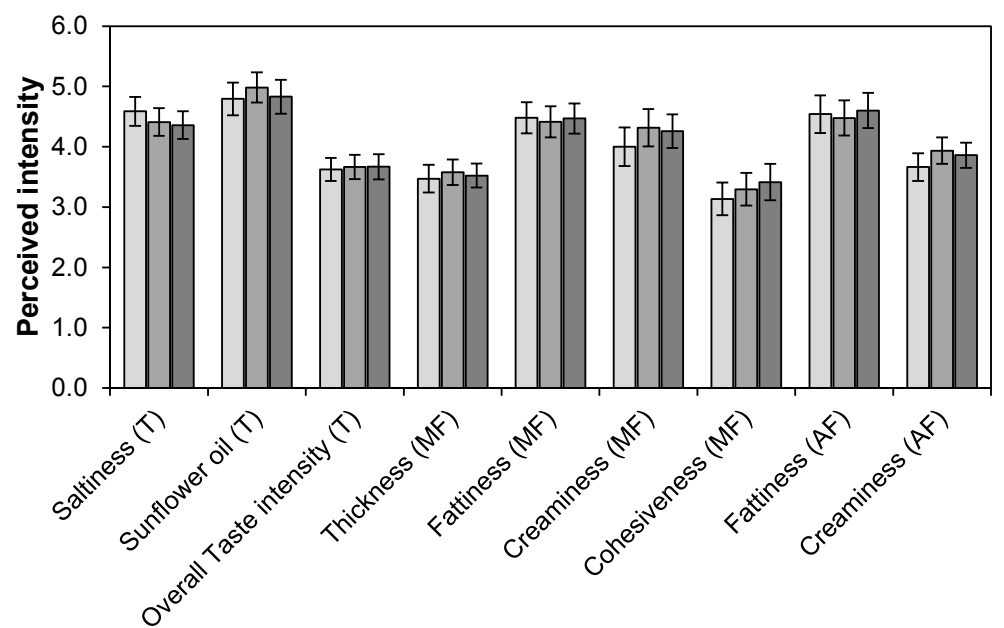

Figure 5.6 Comparison of sensory profiles (mean intensities of all sensory attributes) of WOW 9-21-70 G (light grey), WOW 12-18-70 G (medium grey), and WOW 15-15-70 G (dark grey). Mean intensities of sensory attributes did not differ significantly between samples. Error bars indicate standard errors.

\subsubsection{Effect of fat reduction level on sensory perception of double $\left(w_{1} / 0 / w_{2}\right)$ emulsions with a gelled $w_{1}$ phase}

To investigate the effect of the inner water droplets further, we investigated the sensory perception of emulsions with different fat reduction levels, namely 30, 40, and $50 \mathrm{wt} \%$. As described previously, the level of fat reduction (amount of inner water droplets) can only be increased when the inner aqueous phase are gelled. Since during the preparation of the emulsions, part of the inner dispersed phase is expelled into the outer water phase, the actual fat reductions are slightly lower (Table 5.3) than the aimed fat reduction levels. Figure $\mathbf{5 . 6}$ shows the comparison of the emulsions with 30 vol\% dispersed phase fraction and different fat reduction levels. Mean intensities of sensory attributes did not differ significantly for the emulsions when the gelled inner aqueous phase was increased (higher fat reduction level). Similar results were seen for double emulsions with 50 vol\% dispersed phase (results not shown). It seems that introducing a gelled $w_{1}$ phase leads to the largest differences in oil droplet characteristics, but increasing the amount of gelled inner $\mathrm{w}_{1}$ phase does not seem to further influence the oil droplet characteristics and therefore sensory perception. Even though Dresselhuis and co-workers [24] found for single emulsions a correlation between oils with higher viscosities and lower friction forces, resulting in enhanced sensory perception, we did not observe a similar effect for the double emulsions studied here, even though viscosities of primary $\left(w_{1} / 0\right)$ emulsions increased significantly. Viscosities of the primary $\left(w_{1} / 0\right)$ emulsions increased from 
about $190 \mathrm{mPa} . \mathrm{s}$ for $\left(\mathrm{w}_{1} / \mathrm{o}\right)$ emulsions with $30 \mathrm{wt} \%$ aqueous phase (both gelled and non-gelled) to $345 \mathrm{mPa} . \mathrm{s}$ for ( $\left.\mathrm{w}_{1} / \mathrm{o}\right)$ emulsions with $40 \mathrm{wt} \%$ gelled aqueous phase, to $800 \mathrm{mPa} . \mathrm{s}$ for $\left(\mathrm{w}_{1} / \mathrm{o}\right)$ emulsions with $50 \mathrm{wt} \%$ gelled aqueous phase. If changes in viscosity of the dispersed phase (thus in our case the primary $\left(w_{1} / 0\right)$ emulsion) led to changes in friction, we would expect to see a further enhancement of perceived fat-related sensory attributes. However, we did not find any changes in sensory perception for an increasing fraction of inner gelled $w_{1}$ phase.

To summarize, our results indicate that up to $47 \%$ of the oil in emulsions can be replaced by gelled inner water droplets without changing sensory properties.

\subsection{Conclusions}

The aim of the study was to investigate the effect of introducing small water droplets inside the oil droplets of an $\left(\mathrm{o} / \mathrm{w}_{2}\right)$ emulsion on its sensory properties, in particular fat-related sensory attributes. No or only very small differences in sensory perception between full-fat single $\left(\mathrm{o} / \mathrm{w}_{2}\right)$ emulsions and fat-reduced double $\left(\mathrm{w}_{1} / \mathrm{o} / \mathrm{w}_{2}\right)$ emulsions with a non-gelled inner aqueous phase were observed. Interestingly, the sensory attribute 'fattiness' did not depend in either of the sets of two emulsion on the level of fat reduction. This suggests that fattiness perception depends on total oil droplet surface area rather than bulk properties of the oil droplets. Sensory perception of fatrelated mouth-feel and after-feel attributes was enhanced when the inner aqueous phase was gelled (with gelatin as a gelling agent), indicating that the presence of inner water droplets and their composition play a role in sensory perception. The level of fat reduction can be varied by gelling $w_{1}$ without influencing sensory perception. Our results indicate that a fat reduction of up to $47 \mathrm{wt} \%$ is feasible by means of double $\left(w_{1} / o / w_{2}\right)$ emulsions with a gelled inner aqueous phase. Additional studies with different model emulsions, e.g. removing gelled droplets from the outer water phase, or studying only single $\left(\mathrm{o} / \mathrm{w}_{2}\right)$ emulsions with added gelled droplets in the outer water phase $\mathrm{w}_{2^{\prime}}$ as well as investigations of lubrication properties of the studied emulsions might help to explain the underlying mechanisms of the sensory perception of these emulsions further.

We conclude that significant amounts of oil can be reduced in double emulsions while maintaining or even enhancing perception of fat-related sensory attributes. These results are promising and confirm the potential of double emulsions as oil replacers, opening avenues for future research in fat reduction strategies. 


\section{Acknowledgements}

The study has received funding from the European Union Seventh Framework Programme (FP7/2007-2013) under grant agreement nr. 289397 (TeRiFiQ project). The authors would like to thank Maria Montero Diaz for her contribution to the preparation and characterisation of the emulsions and other products used during the sensory study, and her help during the training and profiling sessions. Rosan van der Glas is thanked for leading the panel discussions and training the panellists. 


\section{References}

1. Muschiolik, G., Multiple emulsions for food use. Current Opinion in Colloid \& Interface Science, 2007. 12: p. 213-220.

2. Jiménez-Colmenero, F., Potential applications of multiple emulsions in the development of healthy and functional foods. Food Research International, 2013. 52: p. 64-74.

3. McClements, D.J., et al., Structural Design Principles for Delivery of Bioactive Components in Nutraceuticals and Functional Foods. Critical Reviews in Food Science and Nutrition, 2009. 49: p. 577-606.

4. Chiu, N., et al., Programmed emulsions for sodium reduction in emulsion based foods. Food \& Function, 2015. 6: p. 1428-1434.

5. Lad, M., L. Hewson, and B. Wolf, Enhancing saltiness in emulsion based foods. Flavour, 2012. 1: p. 1-8.

6. Malone, M.E., I.A.M. Appelqvist, and I.T. Norton, Oral behaviour of food hydrocolloids and emulsions. Part 2. Taste and aroma release. Food Hydrocolloids, 2003. 17: p. 775-784.

7. Chung, C., et al., Reduced Fat Food Emulsions: Physicochemical, Sensory, and Biological Aspects. Critical Reviews in Food Science and Nutrition, 2015. 56: p. 650-685.

8. Akhtar, M., et al., Factors affecting the perception of creaminess of oil-in-water emulsions. Food Hydrocolloids, 2005. 19: p. 521-526.

9. Benjamins, J., et al., Partial coalescence as a tool to control sensory perception of emulsions. Food Hydrocolloids, 2009. 23: p. 102-115.

10. Camacho, S., et al., Formation, Clearance and Mouthfeel Perception of Oral Coatings Formed by Emulsion-Filled Gels. Journal of Texture Studies, 2015. 46: p. 399-410.

11. van Aken, G.A., M.H. Vingerhoeds, and R.A. de Wijk, Textural perception of liquid emulsions: Role of oil content, oil viscosity and emulsion viscosity. Food Hydrocolloids, 2011. 25: p. 789-796.

12. Vingerhoeds, M.H., et al., How emulsion composition and structure affect sensory perception of low-viscosity model emulsions. Food Hydrocolloids, 2008. 22: p. 631-646.

13. Schuch, A., K. Köhler, and H.P. Schuchmann, Differential scanning calorimetry (DSC) in multiple W/O/W emulsions. Journal of Thermal Analysis and Calorimetry, 2013. 111: p. 1881-1890.

14. Stone, H. and J.L. Sidel, Sensory Evaluation Practices. 3rd ed. 2004, Orlando, FL: Academic Press.

15. Stone, H., et al., Sensory evaluation by Quantitative Descriptive Analysis. Food Technology, 1974. 28 : p. 24.

16. Chojnicka-Paszun, A., H.H.J. de Jongh, and C.G. de Kruif, Sensory perception and lubrication properties of milk: Influence of fat content. International Dairy Journal, 2012. 26: p. 15-22.

17. van Aken, G.A., et al., Aspects of sensory perception of food emulsions thickened by polysaccharides, in Gums and Stabilisers for the food industry 13, P.A. Williams and G.O. Phillips, Editors. 2006, Cambridge: Royal Society of Chemistry. p. 449-456.

18. Guinard, J.-X. and R. Mazzucchelli, The sensory perception of texture and mouthfeel. Trends in Food Science \& Technology, 1996. 7: p. 213-219.

19. Naes, T., P. Brockhoff, and O. Tomic, Statistics for Sensory and Consumer Science. 2010: John Wiley and Sons Ltd.

20. Husson, F., et al., FactoMineR: multivariate exploratory data analysis and data mining with $R$. $\mathrm{R}$ package version 1.24, 2013.

21. Schuch, A., et al., Production of W/O/W (water-in-oil-in-water) multiple emulsions: droplet breakup and release of water. Journal of Colloid and Interface Science, 2013. 402: p. 157-64.

22. Bonnet, M., et al., Influence of the oil globule fraction on the release rate profiles from multiple W/O/W emulsions. Colloids and Surfaces B: Biointerfaces, 2010. 78: p. 44-52.

23. Schuch, A., J. Wrenger, and H.P. Schuchmann, Production of W/O/W double emulsions. Part II: Influence of emulsification device on release of water by coalescence. Colloids and Surfaces A: Physicochemical and Engineering Aspects, 2013. 461: p. 344-351.

24. Dresselhuis, D.M., et al., Tribology of $\mathrm{o} / \mathrm{w}$ Emulsions Under Mouth-like Conditions: Determinants of Friction. Food Biophysics, 2007. 2: p. 158-171.

25. Devezeaux de Lavergne, M., et al., Dynamic texture perception and oral processing of semi-solid food gels: Part 1: Comparison between QDA, progressive profiling and TDS. Food Hydrocolloids, 2015. 43: p. 207-217.

26. Kokini, J.L., The physical basis of liquid food texture and texture-taste interactions. Journal of Food Engineering, 1987. 6: p. 51-81. 


\section{Appendix}

Appendix 5.1 Description of reference products used during the training sessions

\begin{tabular}{|c|c|c|c|}
\hline \multirow{2}{*}{ Categories } & \multirow{2}{*}{ Attribute } & \multicolumn{2}{|c|}{ Reference products } \\
\hline & & Type of product & Concentrations $(\%(w / w))$ \\
\hline \multirow{5}{*}{ Taste } & $\begin{array}{l}\text { Sunflower oil } \\
\text { taste }\end{array}$ & Sunflower oil & Pure \\
\hline & & & $0.12 \mathrm{NaCl}$ in $\mathrm{w}_{2}$ \\
\hline & & $\mathrm{O} / \mathrm{W}_{2}$ emulsion with $30 \mathrm{wt} \%$ oil and & $0.17 \mathrm{NaCl}$ in $\mathrm{w}_{2}$ \\
\hline & salty & different $\mathrm{NaCl}$ concentrations in $\mathrm{w}_{2}$ & $0.21 \mathrm{NaCl}$ in $\mathrm{w}_{2}$ \\
\hline & & & $0.25 \mathrm{NaCl}$ in $\mathrm{w}_{2}$ \\
\hline \multirow{6}{*}{ Mouth feel } & & & 0.10 xanthan in $w_{2}$ \\
\hline & Thirknecs & Xanthan aum colutions * & 0.20 xanthan in $w_{2}$ \\
\hline & & Adminan gum solutions & 0.30 xanthan in $\mathrm{w}_{2}$ \\
\hline & & & 0.40 xanthan in $\mathrm{w}_{2}$ \\
\hline & & $\mathrm{O} / \mathrm{W}_{2}$ emulsion (low cohesiveness) & OW 26-74 \\
\hline & Cohesiveness & $\begin{array}{l}\mathrm{W}_{1} / \mathrm{O} / \mathrm{W}_{2} \text { emulsion (high } \\
\text { cohesiveness) }\end{array}$ & WOW 25-25-50 G \\
\hline \multirow{8}{*}{$\begin{array}{l}\text { Mouth feel and } \\
\text { After feel }\end{array}$} & & & $20 \%$ oil \\
\hline & Fattv & $\mathrm{O} / \mathrm{W}_{2}$ emulsions with various & $30 \%$ oil \\
\hline & ratty & dispersed oil phases & $40 \%$ oil \\
\hline & & & $50 \%$ oil \\
\hline & & & $0.00 \%$ xanthan in $\mathrm{w}_{2}$ \\
\hline & Cronmus & $\mathrm{O} / \mathrm{W}_{2}$ emulsion with $30 \%$ oil and & $0.04 \%$ xanthan in $\mathrm{w}_{2}$ \\
\hline & creamy & $\begin{array}{l}\text { altrerent xanthan gum } \\
\text { concentrations in } w_{2}\end{array}$ & $0.10 \%$ xanthan in $\mathrm{w}_{2}$ \\
\hline & & & $0.20 \%$ xanthan in $w_{2}$ \\
\hline
\end{tabular}

* Xanthan gum (Keltrol ${ }^{\circledR}$ Advanced Performance) was kindly provided by CP Kelco (Shandong, China). 


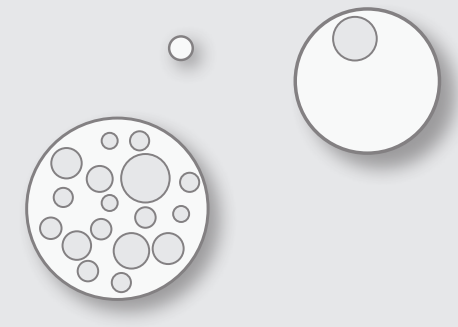

\section{Chapter 6}

\section{Comparison of Rate-All-That-Apply (RATA) and Descriptive sensory Analysis (DA) of model double emulsions with subtle perceptual differences}
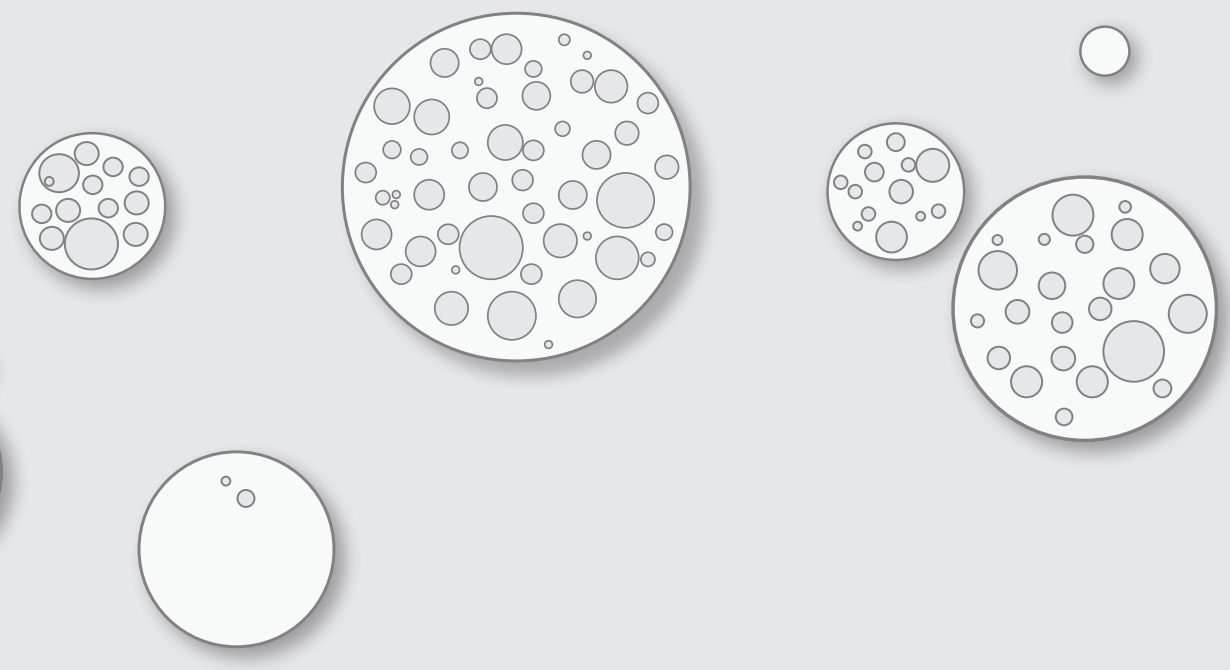

This chapter has been published as

AKL Oppermann, C de Graaf, E Scholten, M Stieger, B Piqueras-Fiszman, Comparison of Rate-All-That-Apply (RATA) and Descriptive sensory Analysis (DA) of model double emulsions with subtle perceptual differences; Food Quality and Preference (2017), 56, 55-68 


\section{Abstract}

The Rate-All-That-Apply (RATA) method, an intensity-based Check-All-That-Apply (CATA) variant, has recently been developed for sensory characterization involving untrained panellists. The aim of this study was to investigate the sensory profiles of ten model (double) emulsions with subtle perceptual differences obtained from the Rate-All-That-Apply (RATA) method with untrained panellists $(n=80)$. For this purpose two different analysis approaches were followed (treating the data as frequencies and as intensities) and then compared to results obtained from Descriptive Analysis (DA) with trained panellists $(n=11)$. The RATA method was adapted by including a short familiarization session to acquaint participants with the RATA methodology, the use of the scale, the sensory terms, and product differences. The comparison involved discriminative ability and configuration similarity by means of Multiple Factor Analysis (MFA) and $R_{v}$ coefficients.

The results in our study show that the RATA intensity approach resulted in higher discriminative ability compared to the RATA frequency approach. Both RATA frequency and RATA intensity resulted in similar overall configurations compared to DA. However, important differences between the use of RATA and DA scales suggest that these overall similarities should be interpreted with caution and warrant a deeper investigation on how RATA scales are understood and used by consumers. 


\subsection{Introduction}

Generic Descriptive Analysis (DA) with trained panels has been widely used to profile sensory properties of foods and beverages since it provides detailed, consistent, and reliable results [1]. However, the economic and time consuming aspect of training a sensory panel can be an issue for academic organizations and food industry [2]. Therefore, several consumer-based sensory profiling methodologies have been developed in sensory testing as more rapid and flexible alternatives to DA [3]. Reduced time investment, costs, and training requirements in combination with consumers describing the sensory properties of products instead of analytically trained subjects are key advantages. Consumer-based sensory profiling methodologies can be based on the evaluation of global differences (e.g., sorting and Napping ${ }^{\circ}$ ), the comparison with product references (polarized sensory positioning), global description of individual products (open-ended questions), and on the evaluation of individual terms (e.g., free choice profiling, flash profiling, and Check-All-That-Apply (CATA) [2]. For sensory product profiling, also attribute-based methods like intensity scaling, Just-About-Right tasks and Ideal Profile Method have been explored with untrained panellists to indirectly or directly provide sensory profiles [2].

With the CATA method, consumers are provided with a checklist of predefined terms and asked to select all those terms that apply to describe a given product [4]. Previous studies have shown that CATA provides reliable product descriptions comparable to those generated by trained panellists $[5,6]$. However, the binary response of CATA does not allow a direct measurement of the intensity of the evaluated sensory terms. As elaborated by Ares and co-workers [7], this could hamper detailed descriptions and discrimination between products with similar sensory properties. Therefore, Ares and co-workers [8] recommended against the use of CATA questions with untrained consumers for sensory profiling of products with small sensory differences. In this case, CATA questions used by untrained consumers may not provide equivalent information as DA with trained panellists. Recently, Rate-All-That-Apply questions (RATA) have evolved from CATA questions by including intensity ratings of the terms that have been selected. Across a number of studies, Ares and co-workers [7] showed that RATA questions, in comparison to CATA, led to an increase in the total number of selected terms. However, even though the total number of selected terms increased, the percentage of selected terms that significantly discriminated the products only increased slightly. Reinbach and co-workers [9] even found a decrease in significant differences when RATA was used, which could be due to the lack of training in rating the intensity of terms and therefore inconsistent use of scales, leading to variability 
in consumers' ratings. Giacalone and Hedelund [10] recently investigated the reproducibility at assessor-, attribute- and panel-level of the RATA methodology with semi-trained assessors. Assessors were trained during four sessions and subsequently evaluated chocolate samples in quadruplicate. They found that within-assessor reproducibility was moderate and that product maps obtained from individual replicates at panel-level showed high configurational agreement.

It is also worth noting that reported RATA studies so far have involved generally familiar foods such as beer, bread, gummy lollies, peanuts, and apples $[7,9,11]$. These products are relatively easy for consumers to profile. However, it is unclear whether RATA results with untrained panellists would be similar to DA results with trained panellists, or how results from RATA as frequencies of selection only (CATA) would compare to results from RATA as intensities, when non-commercial stimuli such as emulsions with very subtle differences are used.

The food stimuli of interest in the present study are model food emulsions. Model food emulsions can be engineered to precisely control their physical properties and are frequently used to assess the influence of physical properties on sensory perception [12-14]. Double $\left(w_{1} / o / w_{2}\right)$ emulsions are particularly interesting for healthier product formulations since they have potential for fat reduction by introducing small water droplets $\left(w_{1}\right)$ inside oil droplets that are dispersed in a continuous water phase $w_{2}$. The total interfacial area between oil droplets and outer water phase $w_{2}$ is similar for full-fat $\left(\mathrm{o} / \mathrm{w}_{2}\right)$ emulsions and double $\left(\mathrm{w}_{1} / \mathrm{o} / \mathrm{w}_{2}\right)$ emulsions while reducing total fat content. This makes the study of different double emulsion formulations interesting from a sensory perspective. However, the preparation of double emulsions entails the challenge of stabilizing the small $w_{1}$ droplets inside the oil droplets. The level of fat reduction that can be achieved by the introduction of inner water droplets is limited, since more pronounced contact between the small $w_{1}$ droplets increases the probability of coalescence with the outer water phase $\left(w_{2}\right)$ during the preparation of the double emulsions. This risk of coalescence can be reduced by gelling those inner water droplets, thereby increasing the possible level of fat reduction [15]. In a previous study, we recently investigated the sensory perception of ten reducedfat double emulsions with a trained sensory panel (chapter 5). The emulsions varied in the level of fat reduction and composition (gelled/non-gelled) of the inner $w_{1}$ phase and were compared to a full-fat reference sample. The study showed that fat-related sensory perception between full-fat emulsions and fat-reduced double emulsions was very similar, with small significant differences detected for the sensory term creaminess. Double emulsions with a gelled inner $w_{1}$ phase further enhanced 
thickness and cohesiveness perception compared to non-gelled versions. Fattiness perception did not differ across emulsions, which was attributed to the total interfacial area that remained the same. Small changes in sensory perception were assumed to be partly attributed to changes in lubrication properties. Overall, we concluded that the differences between stimuli were subtle.

The motivation of the current research is to investigate whether untrained panellists could also discriminate such a set of non-commercial samples with subtle perceptual differences of a generally unfamiliar product category.

The main aim of this study was therefore to compare different approaches to analyze RATA data of samples with subtle differences and to investigate whether data from untrained subjects performing RATA can lead to the same overall general conclusions as data obtained by a trained panel. In contrast to Giacalone and Hedelund [10], who trained a panel of employees with considerable product expertise during four sessions, we used one familiarization session only to acquaint naive subjects with the RATA methodology, the use of the scale, and the samples. Intensity rating was implemented by asking participants to rate the intensities for applicable terms on a 9-box scale.

In order to fully investigate the data and compare results to similar studies [7, 11], RATA data were explored by the frequency of term selection only (treated as CATA data and not taking into account the intensity ratings of selected terms) as well as their intensity scores. Sensory profiles of the model (double) emulsions obtained by DA and RATA (both approaches) were compared for: (1) discriminative ability between methods, and (2) configuration similarity by means of Multiple Factor Analysis (MFA) and $R_{v}$ coefficients, as well as by inspection of individual configurations.

\subsection{Materials and methods}

\subsubsection{Samples}

Two sets of emulsions with either 30 or $50 \%$ dispersed phase were evaluated. Each set comprised of five model food emulsions differing in the degree of fat reduction. The dispersed phase was either oil droplets or oil droplets filled with varying amounts of (gelled) water droplets, hence various levels of fat reduction (see Figure 6.1). Double emulsions were prepared in a two-step process and the process is described in more detail in chapter $\mathbf{5}$. First, primary $\left(w_{1} / 0\right)$ emulsions were prepared by mixing 30,40 or $50 \mathrm{wt} \%$ inner water phase containing $0.4 \mathrm{wt} \% \mathrm{NaCl}$ with sunflower oil containing a lipophilic emulsifier (polyglycerol polyricinoleate, PGPR) in a high shear 
blender (Waring blender 8011 ES, Stamford, CT). In case of gelled water droplets, gelatin (10 wt\%) was added to the inner water phase, and gelation was induced by subsequent cooling a heated emulsion. Double emulsions were prepared by dispersing $30 \mathrm{wt} \%$ of the primary $\left(w_{1} / 0\right)$ emulsions in $70 w t \%$ of the outer water phase containing $1 \mathrm{wt} \%$ whey protein isolate as hydrophilic emulsifier and $0.2 \mathrm{wt} \% \mathrm{NaCl}$ using a high shear blender (Ultra Turrax T25 with the dispersing tool S25-N 18G, IKA, Staufen, Germany). Mixing speeds during preparation were adapted to obtain emulsions with similar oil droplet sizes. Figure 6.1 and Table $\mathbf{6 . 1}$ provide an overview of the emulsion characteristics. As explained in chapter 5, sample names indicate the emulsion type ("OW" for single emulsions, "WOW" for double emulsions), the amount of inner aqueous phase $w_{1}$, oil phase and outer water phase $w_{2}$ in the final emulsion, followed by the physical state of the inner aqueous phase ("NG" for non-gelled, "G" for gelled). For example, the label "WOW 12-18-70 G" refers to a double emulsion containing $12 \mathrm{wt} \%$ of gelled w 1 droplets dispersed in $18 \mathrm{wt} \%$ of oil, further dispersed in $70 \mathrm{wt} \%$ of outer continuous water phase $w_{2}$. The $12 \mathrm{wt} \%$ of gelled inner aqueous phase $w_{1}$ and $18 \mathrm{wt} \%$ of oil correspond to $30 \mathrm{wt} \%$ of dispersed $\left(w_{1} / 0\right)$ phase. Since a small fraction of the inner water droplets is expelled to the outer water phase during preparation, the real mass fractions of the three phases are slightly different. The real dispersed phase $\left(w_{1} / 0\right)$ fractions were determined by measuring the yield, i.e. the amount of $w_{1}$ droplets remaining inside the oil droplets after emulsion preparation. The real dispersed phase mass fractions are listed in Table 6.1. As a reference, full-fat single $\left(0 / w_{2}\right)$ emulsions with the same real total dispersed phase mass fraction were prepared. A "WOW 12-18-70 G" emulsion corresponds therefore to a single "OW 26-74" emulsion, representing a fat reduction level of $40 \%$ in the double emulsion. Single (o/ $\mathrm{w}_{2}$ ) emulsions do not contain an inner aqueous phase, therefore, only the amount of dispersed oil phase (26 or $47 \mathrm{wt} \%$ ) and outer water phase (74 or $53 \mathrm{wt} \%$ ) are denoted in the sample names. Emulsion preparation took place in a food safe environment.

\subsubsection{Sensory evaluations}

The sensory properties of the emulsions were evaluated by a trained sensory panel according to the principles of quantitative descriptive analysis $[1,16]$, as well as by an untrained panel using the RATA method. In both studies, an informed consent was signed by all participants at an information session to confirm the awareness of the presence and possible risks of polyglycerol polyricinoleate (PGPR), the lipophilic emulsifier used to stabilize (gelled) water droplets inside the oil droplets in some of the samples. Participants were compensated for their participation. Both studies were approved for conduct by the Wageningen University research ethics committee (registered under NL50244.081.14). 


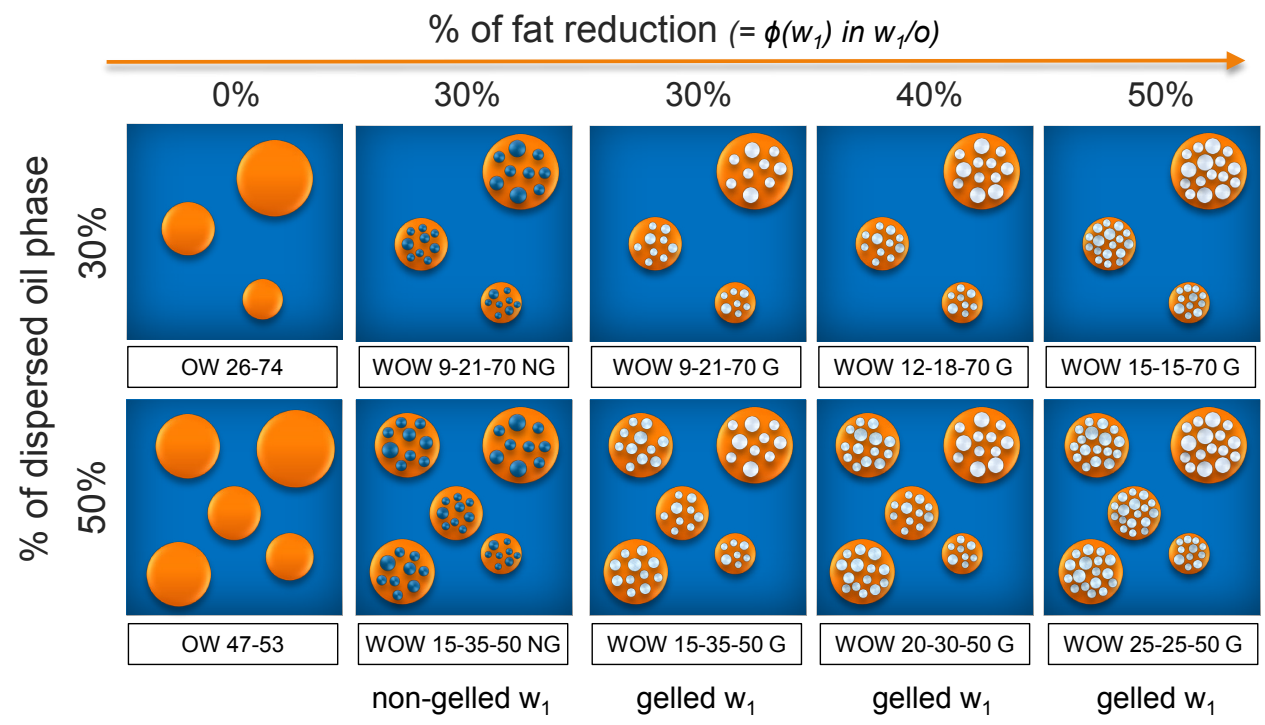

Figure 6.1 Schematic overview of ten emulsions assessed by RATA and DA, based on the study design described in chapter 5 . In the upper row, emulsions contain approximately $30 \%$ of dispersed phase. OW 26-74 refers to $0 \%$ fat reduction and WOW $15-15-70 \mathrm{G}$ to $50 \%$ fat reduction, and the oil droplets contain non-gelled (NG) or gelled (G) water droplets. In the lower row, emulsions contain about $50 \%$ of dispersed phase, in which between $0 \%$ (OW 47-53) and $50 \%$ (WOW 25-25-50 G) of oil has been replaced by either non-gelled (NG) or gelled (G) water droplets.

Table 6.1 Emulsion compositions based on the study design described in chapter $5 . \Phi\left(w_{1}\right)$ corresponds to the level of fat reduction, i.e. how much oil is replaced by (gelled) water droplets. "Gelatin" corresponds to the amount of gelatin in the inner water phase $w_{1}$. Real $\Phi\left(\right.$ oil) or $\Phi\left(w_{1} / 0\right)$ correspond to the real amounts of dispersed (oil) phase in the final emulsion and differ slightly from theoretical values based on the calculated yields. Oil droplet size is the size of the oil droplets or oil droplets including (gelled) inner water droplets.

\begin{tabular}{llllll}
\hline Label & Emulsion type & $\begin{array}{l}\Phi\left(w_{1}\right)[w t \%] \\
\text { in } w_{1} / 0\end{array}$ & $\begin{array}{l}\text { Gelatin } \\
{\left[g / 100 g w_{1}\right]}\end{array}$ & $\begin{array}{l}\text { Real } \Phi(o i l) \text { or } \\
\Phi\left(w_{1} / o\right)[w t \%]\end{array}$ & $\begin{array}{l}\text { Oil droplet size } \\
\mathbf{d}_{4,3}[\mu \mathrm{m}]\end{array}$ \\
\hline OW 26-74 & $\mathrm{O} / \mathrm{W}_{2}$ & 0 & 0 & $26.0 \pm 0.0$ & $52.5 \pm 3.7$ \\
WOW 9-21-70 NG & $\mathrm{W}_{1} / \mathrm{O} / \mathrm{W}_{2}$ & 30 & 0 & $25.6 \pm 0.4$ & $51.6 \pm 1.7$ \\
WOW 9-21-70 G & $\mathrm{W}_{1} / \mathrm{O} / \mathrm{W}_{2}$ & 30 & 10 & $28.6 \pm 0.3$ & $49.9 \pm 3.9$ \\
WOW 12-18-70 G & $\mathrm{W}_{1} / \mathrm{O} / \mathrm{W}_{2}$ & 40 & 10 & $27.5 \pm 0.3$ & $49.0 \pm 2.7$ \\
WOW 15-15-70 G & $\mathrm{W}_{1} / \mathrm{O} / \mathrm{W}_{2}$ & 50 & 10 & $27.1 \pm 0.7$ & $52.9 \pm 1.7$ \\
OW 47-53 & $\mathrm{O} / \mathrm{W}_{2}$ & 0 & 0 & $47.0 \pm 0.0$ & $64.6 \pm 2.5$ \\
WOW 15-35-50 NG & $\mathrm{W}_{1} / \mathrm{O} / \mathrm{W}_{2}$ & 30 & 0 & $44.5 \pm 0.2$ & $59.6 \pm 2.5$ \\
WOW 15-35-50 G & $\mathrm{W}_{1} / \mathrm{O} / \mathrm{W}_{2}$ & 30 & 10 & $48.5 \pm 0.7$ & $58.9 \pm 4.1$ \\
WOW 20-30-50 G & $\mathrm{W}_{1} / \mathrm{O} / \mathrm{W}_{2}$ & 40 & 10 & $48.1 \pm 0.5$ & $55.5 \pm 0.8$ \\
WOW 25-25-50 G & $\mathrm{W}_{1} / \mathrm{O} / \mathrm{W}_{2}$ & 50 & 10 & $47.1 \pm 0.5$ & $58.2 \pm 1.6$ \\
\hline
\end{tabular}

\subsubsection{Descriptive sensory Analysis (DA)}

The DA sensory panel comprised 11 panellists (8 male, 19-54 years old). Generic descriptive analysis (DA) was used for sensory characterization of the emulsions. In total, 18 training sessions of 60 min each took place. Term generation took place during the first four sessions, followed by 14 training sessions during which panellists were trained on the quantification of the selected terms (Table 6.2) using $100 \mathrm{~mm}$ 
unstructured line scales. After the training phase, samples were evaluated using $100 \mathrm{~mm}$ unstructured line scales anchored with "not at all" at the left end and "very" at the right. Samples were coded using 3-digit random numbers and presented following a Williams Latin square design. Four replications of each sample were evaluated by each panellist. Testing took place in a sensory laboratory in standard sensory booths at $20^{\circ} \mathrm{C}$. Between each sample evaluation, panellists had a break of 5 min to cleanse their mouths with warm water $\left(40^{\circ} \mathrm{C}\right)$ and white bread without crust. Panellists used tongue scrapers (budni dent, Hamburg, Germany) to aid the removal of an oral coating after tasting the emulsions. Further details on the training and profiling sessions including the results of this study can be found in chapter 5 .

Table 6.2 Overview of sensory terms used in Descriptive sensory Analysis (DA) and Rate-All-ThatApply (RATA). No definitions are listed for the RATA study since participants were not given any written sensory term definitions.

\begin{tabular}{|c|c|c|c|}
\hline \multirow[t]{2}{*}{ Category } & \multicolumn{2}{|c|}{ Descriptive Sensory Analysis } & \multirow{2}{*}{$\begin{array}{c}\text { Rate-All-That-Apply } \\
\text { Terms }\end{array}$} \\
\hline & Terms & Definitions & \\
\hline \multirow[t]{4}{*}{ Taste (T) } & Saltiness & The taste of a salt solution & Saltiness \\
\hline & $\begin{array}{l}\text { Sunflower oil } \\
\text { Overall taste intensity }\end{array}$ & $\begin{array}{l}\text { The taste of sunflower oil } \\
\text { Overall taste intensity }\end{array}$ & Sunflower oil \\
\hline & & & Dustiness \\
\hline & & & Bitterness \\
\hline \multirow[t]{11}{*}{ Mouth-feel (MF) } & Thickness & $\begin{array}{l}\text { Resistance to flow before saliva } \\
\text { modifies the sample }\end{array}$ & Thickness \\
\hline & Fattiness & $\begin{array}{l}\text { Amount of fat that is perceived when } \\
\text { having the product in one's mouth for } \\
\text { several seconds }\end{array}$ & Fattiness \\
\hline & Creaminess & Soft, velvety and smooth mouth-feel & Creaminess \\
\hline & Cohesiveness & $\begin{array}{l}\text { Compact perception, described as } \\
\text { "product remains as a whole", and "is } \\
\text { not easy to swallow" }\end{array}$ & Cohesiveness \\
\hline & & & Airiness \\
\hline & & & Graininess \\
\hline & & & Mouth-filling \\
\hline & & & Sliminess \\
\hline & & & Smoothness \\
\hline & & & Thinness \\
\hline & & & Stickiness \\
\hline \multirow[t]{6}{*}{ After-feel (AF) } & Fattiness & $\begin{array}{l}\text { The fatty layer that remains in the } \\
\text { mouth }\end{array}$ & Fattiness \\
\hline & Creaminess & $\begin{array}{l}\text { Soft, velvety and smooth perception } \\
\text { in the mouth }\end{array}$ & Creaminess \\
\hline & & & Dryness \\
\hline & & & Roughness \\
\hline & & & Sliminess \\
\hline & & & Stickiness \\
\hline
\end{tabular}

\subsubsection{Rate-All-That-Apply (RATA)}

The RATA assessments were conducted with 80 participants (60 female, 18-65 years). Participants were recruited from the consumer database of the Division of Human 
Nutrition of Wageningen University (the Netherlands), based on their self-reported health, their body weight (above $55 \mathrm{~kg}$ due to the recommended daily intake of the lipophilic emulsifier), and their frequency of consuming fat-containing liquid food products.

One familiarization session of 60 minutes was used to acquaint participants with the model emulsions, the RATA method, the definitions of sensory terms, the use of the 9-box scale, and its anchors to rate the intensity of sensory terms, as well as the cleansing procedure. A 9-box scale was used instead of a 3- or 5-point scale to improve the discriminability of samples with subtle differences [17, 18]. Despite this one familiarization session, here we refer to the panellists as untrained with regard to RATA. Emulsions used in this familiarization session were OW 26-74 and WOW 25-25-50 G, these being the samples with the largest sensorial differences, as well as WOW 15-15-70 G, which was the sample with an intermediate profile. After the familiarization session, participants reported that it was clear to them how to perform the test and use the scales in the evaluation sessions. Prior to the start of the evaluation sessions, they were asked if the procedure was still clear and explained again if necessary.

RATA data were collected on paper in meeting facilities (Agrotechnology and Food Sciences Group, Wageningen University) equipped with individual sensory booths. The RATA questionnaire was developed on the basis of a discussion with a group of 30 consumers, who identified themselves as consumers of fatty, liquid products. Twenty-one terms were selected (Table 6.2), in line with the terms generated during the DA, and with literature describing sensory properties of model, single emulsions [19-21]. Although it is not very common that untrained panels use a more extensive list of terms than trained panels, a need was felt for this particular type of products to use a longer list of sensory terms. The high number of sensory terms resulted from pre-tests, which revealed that these terms were more natural to describe this type of product. It seemed that naïve participants considered the products more difficult to describe than the trained panel, hence the longer list of terms. Terms were randomized within blocks (taste, mouth-feel and after-feel perception) for each participant but not for each emulsion, following a balanced design for presentation order (Williams Latin Square). Emulsions were presented in a monadic sequence. For each term, panellists first selected whether the term applied to describe the sample, and only if so, rated its perceived intensity on a 9-box scale from "low" to "high". Between each sample evaluation, panellists had a break of 5 min to cleanse their mouths with warm water $\left(40^{\circ} \mathrm{C}\right)$ and white bread without crust. Participants also used 
tongue scrapers (budni dent, Hamburg, Germany) to aid the removal of an oral coating after tasting the emulsions. Data was collected in two sessions of 45 minutes each. In the first session, participants evaluated the first set with emulsions containing $30 \%$ dispersed phase. In the second session, participants evaluated the set of emulsions with $50 \%$ dispersed phase. Each participant completed all sessions (familiarization, and both evaluation sessions) within 10 days.

\subsubsection{Statistical data analysis}

Statistical data analysis of the descriptive sensory profiling data followed a standard approach. Panel performance was checked with analyses of variance (ANOVA) on the term ratings considering the sample, panellist (as random effect), replicate, and all their second order interactions as explanatory variables (those with the panellist as random effect). A two-way ANOVA (sample as fixed factor and panellist as random factor) was carried out on the ratings of all ten emulsions to study the perceptual differences among all samples, including the dispersed oil phase fractions (30 \% vs. $50 \%$ ). Then, each set (30 or $50 \%$ dispersed phase) was investigated separately to study the differences within each emulsion set. For this analysis, two-way ANOVAs (sample as fixed factor and panellist as random factor) were carried out for all sensory terms within each set in order to determine significant differences among the samples in terms of taste, mouth-feel, and after-feel. In case of significant differences, multiple pairwise comparisons were performed with Tukey's Honest Significant Difference test (HSD) at $95 \%$ confidence level. Principal Component Analysis was carried out for each emulsion set on the average ratings of the terms to identify relations between samples and terms in the perceptual space.

Two approaches were taken to investigate the RATA data. One approach considered the data as RATA intensity. The other approach, more exploratory, considered the frequency of selection of terms (RATA frequency) to compare the discriminative ability of the two approaches. In terms of intensity scores, RATA data were interpreted as 10-point scales, considering a value of 0 in case the term was not considered applicable to describe a given sample. Based on a follow-up enquiry, most participants indicated that they did not check a term when it was not perceivable at all. A nonselected term was therefore treated equivalent to a "not perceived" label (intensity $=0$ ). RATA intensity scores (0-9) were treated as continuous data, since Meyners and co-workers [11] showed that despite the stepwise setup of the RATA methodology, conclusions from parametric tests are typically very similar to those from nonparametric (Friedman's) tests. Therefore, two-way ANOVAs (with sample as fixed factor and panellist as random factor) were carried out for all sensory terms within 
each set of emulsions to study the differences among the emulsions in terms of taste, mouth-feel, and after-feel. In case of significant differences, post-hoc tests were performed with Tukey's Honest Significant Difference test (HSD) at $95 \%$ confidence level. Principal Component Analyses were carried out on the average ratings of the terms to identify relations between samples and terms.

Frequency of use of each RATA term was determined by counting the number of consumers who used that term; thus, treating the data as CATA (binary) data. Cochran's $Q$ test [22] was carried out for each of the sensory terms within each set of emulsions to identify significant differences among samples. Correspondence analysis (CA) was then performed on the frequency of selection of terms to identify relations between samples and terms.

Multiple Factor Analysis (MFA) [23] was used to assess the configurational similarity of product spaces obtained with each statistical approach regarding the RATA data (RATA frequency and RATA intensity) and DA methodology. Given the difference in number of sensory terms, only the eight terms used in both RATA and DA were subjected to the MFA for a direct statistical comparison. The similarity of product spaces was evaluated through sample space and partial point representations. Regressor vector $\left(R_{v}\right)$ coefficients [24] were calculated to quantitatively measure configurational congruency. In this study, $R_{v}$ coefficients were calculated for all possible combinations of methodologies (RATA frequency, RATA intensity and DA) for the first two dimensions. The significances of the $R_{v}$ coefficients were calculated with the Pearson type III approximation [25]. High $R_{v}$ coefficient values would indicate that the methods yield similar information.

All statistical analyses were performed using $\mathrm{R}$ language (version 3.2.3). FactoMine $R$ package [26] was used to perform Multiple Factor Analysis, confidence ellipses around products in the Correspondence Analysis (ellipseCA function) and calculation of $R_{v}$ coefficients [27], while the RVAideMemoire package was used to perform Cochran's $\mathrm{Q}$ test. Panellipse function in SensoMineR was used to display confidence ellipses around products in Principal Component Analysis.

\subsection{Results and discussion}

\subsubsection{Discriminative ability of RATA data analysed based on frequencies and intensities}

First, the discriminative ability of the RATA method with data analysed as frequency of term selection (RATA frequency) was determined and compared to that when RATA data were analysed as intensity scores (RATA intensity). RATA data analysed as 
frequencies was investigated with Cochran's $Q$ test to determine significant differences among emulsions for each of the sensory terms, while RATA intensities were analysed with ANOVAs for each of the sensory terms ${ }^{1}$. Results are summarized in Table 6.3. The sum of sensory terms that were significantly different among emulsions is presented at the bottom of the table.

When considering the RATA data as binary data (frequencies), significant differences among emulsions with $30 \%$ dispersed phase were identified in 6 of the 21 terms (see Table 6.3). For instance, the frequency with which the term 'thin (MF)' was selected decreased with increasing level of fat reduction: the term was selected by $64 \%$ of the participants for the full-fat emulsion (OW 26-74), while the frequency of selection decreased to $40 \%$ for the emulsion WOW 15-15-70 G. In contrast, frequency of selection for the term creamy (MF) increased with increasing level of fat reduction: the term was selected by $54 \%$ of the participants for the emulsion OW 26-74 in comparison to $73 \%$ for the emulsion WOW 15-15-70 G (see Appendix 6.1). In comparison, significant differences among emulsions with $50 \%$ dispersed phase were identified in 5 of the 21 terms. When treating the data as RATA intensities in contrast to analysing RATA data as frequencies, 8 and 10 of the 21 terms significantly discriminated emulsions with 30 and $50 \%$ dispersed phase, respectively. This difference in the number of discriminating terms found between the two approaches to analyse RATA data (as frequency or intensity data) contrasts results of previous studies $[7,11]$ where such a difference was not observed. Four possible reasons for this divergence from previous studies can be considered. One possible reason accounts the degree of perceptual differences between emulsions. In the case samples are considerably different, different terms are used to describe the sensory profile of samples. In that case, it is likely that differences between RATA intensities and RATA frequencies are not found. However, in our study, model emulsions had a small degree of perceptual differences. Similar terms applied to describe the emulsions, and the probability that differences are observed within the two approaches increases. The average number of terms selected for each of the emulsions did not differ significantly within each set of emulsions $(\mathrm{n}=7.5, \mathrm{~F}=0.88, p=0.478$ for emulsions with $30 \%$ dispersed phase; $\mathrm{n}=7.9, \mathrm{~F}=0.87, p=0.485$ for emulsions with $50 \%$ dispersed phase). However, more sensory terms were found to be significantly different between emulsions since their perceived intensities differed. Therefore, analysing RATA data based on their rated intensities, and not only the frequency, had an additional positive effect on the number of discriminating terms to describe emulsions with subtle

1 For completeness, Friedman's tests were also conducted on the RATA intensities for each sensory term. Except for three sensory terms (Rough (AF) and Creamy (AF) for the emulsion set with $30 \%$ dispersed oil phase, and Sunflower oil (T) for the emulsion set with $50 \%$ dispersed oil phase, all conclusions drawn were identical. 


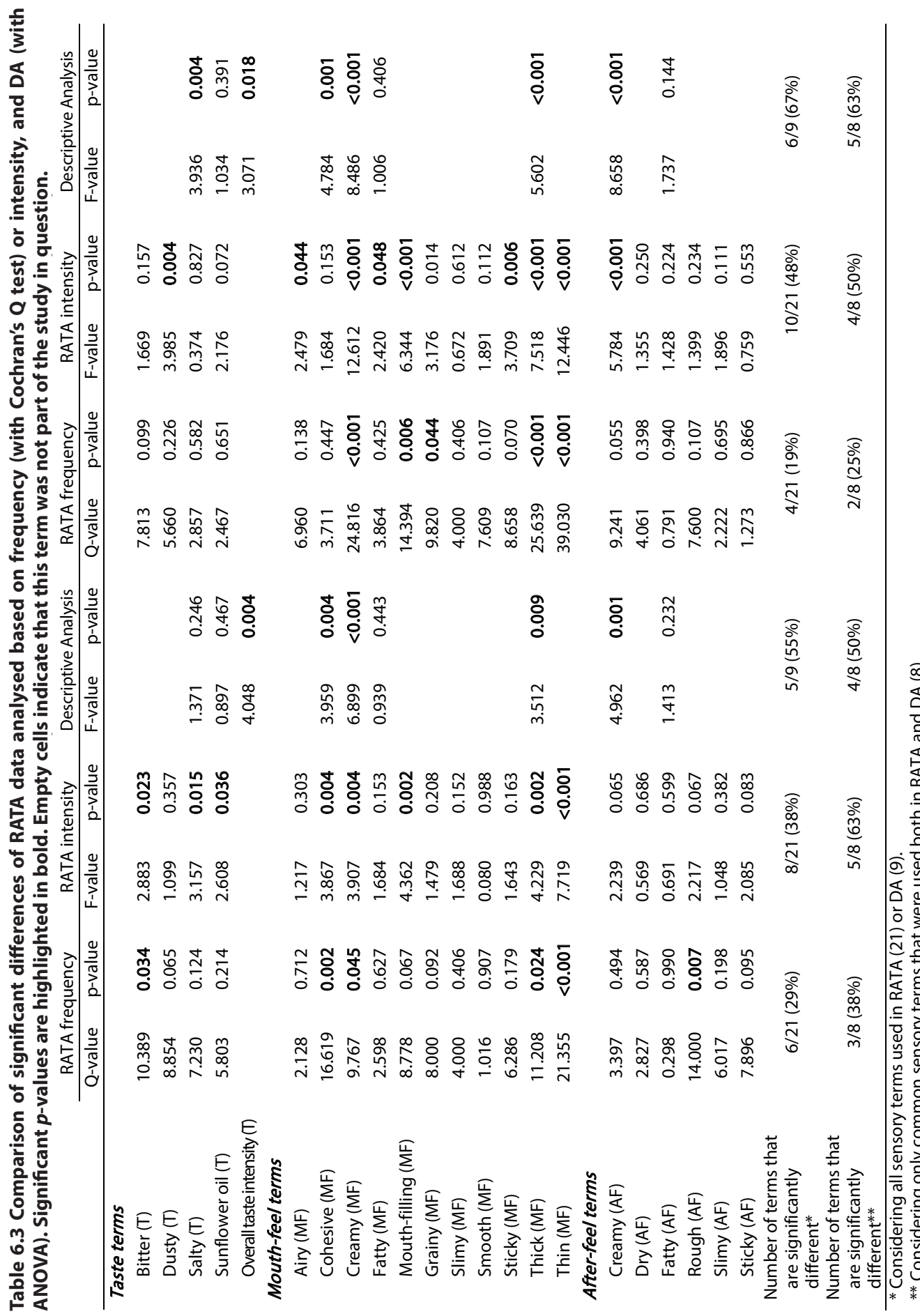


perceptual differences. Also Ares and Jaeger [28] suggested that the CATA method, which only considers the frequency of selected terms, might not be the method of choice if the study aim is to detect small or subtle differences among samples.

The second possible reason for the improved discriminative ability is that we used 9-box scales, whereas Ares and co-workers [7] used 3- or 5-pt scales in their studies. These 3- to 5-pt scales are easier to use by consumers and are efficient if the main aim of the study is to provide some general intervals to express to which extent a sensory term is applicable. In the present study, a 9-box scale is more appropriate as it allows participants to express the perceived intensities in sufficiently smaller steps.

A third possible reason is the inclusion of a familiarization session prior to the RATA evaluation sessions, during which consumers were acquainted with the methodology and the largest perceptual differences to be expected between emulsions. Ares and co-workers [7] did not perform such a familiarization session with the panellists before milk desserts, bread, and gummy lollies were evaluated. Due to this familiarization session, we assume that the panellists were more aware of how subtle the sensory differences between emulsions were and it is likely that panellists paid more attention to small differences in intensity during the evaluation sessions.

A fourth reason is the mathematical computation of the tests. Cochran's $Q$ test to analyse RATA data as frequencies is more sensitive than the ANOVAs used to analyse RATA intensities. Furthermore, allocating a 0 to all terms that had not been checked may decrease the variance within products, and thereby increasing the variability between samples. Therefore, the chance to find significant differences between samples increases.

\subsubsection{Discriminative ability of RATA vs. Descriptive Analysis}

To assess whether untrained participants using the RATA approach reached similar discriminative ability than the trained panel with DA, Table 6.3 compares RATA frequency and RATA intensity with DA regarding the number of sensory terms discriminating the emulsions. Since the number of terms evaluated or used differed between RATA and DA, it is not possible to directly compare the discriminative ability of untrained and trained panellists by comparing the number of terms with significant differences [8]. We therefore compared the discriminative ability of the RATA method with untrained panellists and DA with trained panellists only for the sensory terms that were used in both methodologies. For both sets of emulsions, RATA frequency gave the lowest number of common terms ( 2 and 3 out of 8 ) that were perceived as significantly different in comparison to RATA intensity (4 and 5 terms) and DA 
(also 4 and 5 terms, out of 8). It is important to keep in mind that results will always depend on the type of post-hoc test used [11]. Post-hoc comparisons between RATA intensity and DA show that RATA intensity, in comparison to DA, did not only result in similar significant differences across terms, but also across samples within a term (see Table 6.4).

Table 6.4 Comparison of post-hoc results (Tukey's) for sensory terms which were significantly different in both methodologies (RATA intensity and DA). Within a term and method, different letters represent significant differences among the samples (at $p<0.05$ ).

\begin{tabular}{|c|c|c|c|c|c|c|}
\hline & & \multicolumn{5}{|c|}{ Emulsions with $30 \%$ dispersed phase } \\
\hline & & OW 26-74 & WOW9-21-70NG & WOW9-21-70G & WOW 12-18-70G & WOW 15-15-70G \\
\hline \multirow{2}{*}{ Cohesive (MF) } & DA & $a$ & $a, b$ & $a, b$ & $\mathrm{~b}$ & $\mathrm{~b}$ \\
\hline & RATA & $a, b$ & $a$ & $a, b$ & b & b \\
\hline \multirow{2}{*}{ Creamy (MF) } & DA & $a$ & $a, b$ & b & b & b \\
\hline & RATA & $\mathrm{a}$ & $a, b$ & b & $a, b$ & b \\
\hline \multirow{4}{*}{ Thick (MF) } & DA & $\mathrm{a}$ & $a, b$ & $a, b$ & b & $a, b$ \\
\hline & RATA & $\mathrm{a}$ & $\mathrm{a}$ & $a, b$ & $a, b$ & $\mathrm{~b}$ \\
\hline & & \multicolumn{5}{|c|}{ Emulsions with $50 \%$ dispersed phase } \\
\hline & & OW 47-53 & WOW15-35-50NG & WOW15-35-50G & WOW 20-30-50 G & WOW $25-25-50 \mathrm{C}$ \\
\hline \multirow{2}{*}{ Creamy (MF) } & $\mathrm{DA}$ & $a$ & $a, b$ & $\mathrm{~b}$ & $\mathrm{~b}$ & $\mathrm{~b}$ \\
\hline & RATA & $\mathrm{a}$ & $a, b$ & $b, c$ & c & c \\
\hline \multirow{2}{*}{ Thick (MF) } & DA & a & $a, b$ & $a, b$ & b & $a, b$ \\
\hline & RATA & $a, b$ & a & $b, c$ & $a, b, c$ & c \\
\hline \multirow{2}{*}{ Creamy (AF) } & DA & $\mathrm{a}$ & $a, b$ & b & b & b \\
\hline & RATA & $a$ & $a, b$ & b & $\mathrm{b}$ & $\mathrm{b}$ \\
\hline
\end{tabular}

Considering the data analysis followed, these results suggest that RATA intensity with untrained panellists show similar discriminative ability as DA with trained panellists. These findings support the use of the RATA method to measure the perceived intensity of sensory terms using untrained panellists, particularly when evaluating samples with small or subtle perceptual differences. These findings go beyond results from other studies that reported naive consumers to be able to rate the intensity of simple sensory terms [29-33]. It is possible that the addition of a familiarization session may have already been sufficient for consumers to evaluate complex samples with subtle differences. However, further empirical evidence is needed to support this suggestion.

\subsubsection{Configuration similarity}

\subsubsection{Multiple Factor Analysis (MFA)}

The second step of our analysis aimed at evaluating the configuration similarity of the product spaces obtained by the three data matrices based on the two RATA analysis approaches and DA. To assess the configuration similarity, MFA was performed on three cross tabulation matrices containing frequency of selection for each term 
(RATA frequency), and average ratings of samples (RATA intensity and DA). MFAs were performed only on the eight terms that were used in both studies. Figure $\mathbf{6 . 2}$ shows the first two dimensions of the consensus MFA sample map (84.4\% for the emulsion set with $30 \%$ dispersed phase, Figure $\mathbf{6 . 2 A}$, and $81.6 \%$ for the emulsion set with $50 \%$ dispersed phase, Figure 6.2B). Partial configurations obtained by the individual methods/approaches to analyse data are superimposed onto the consensus points.

Individual factor map

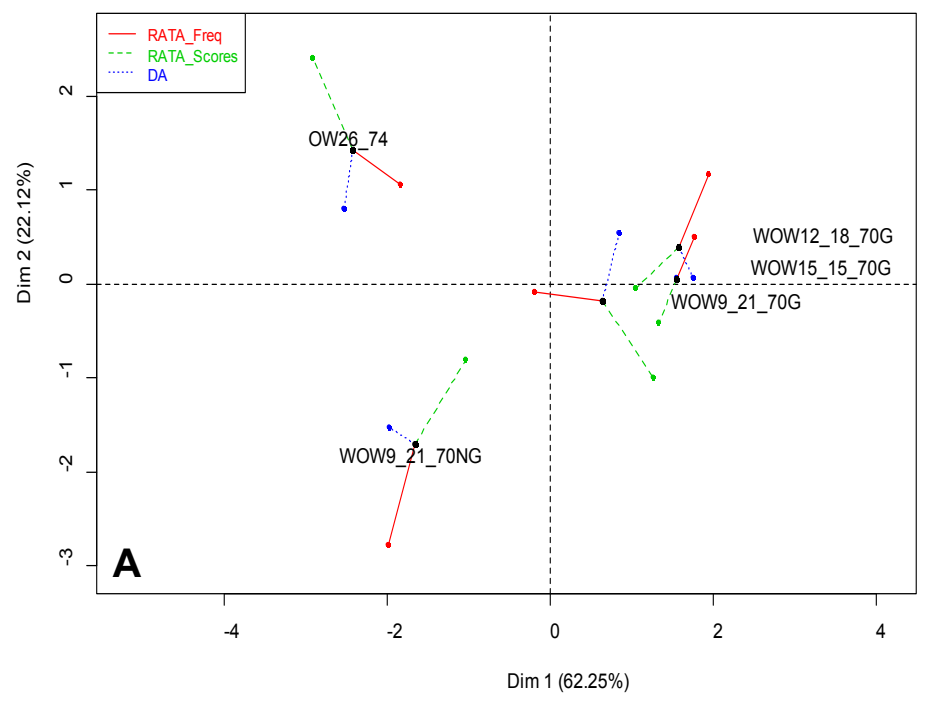

Individual factor map

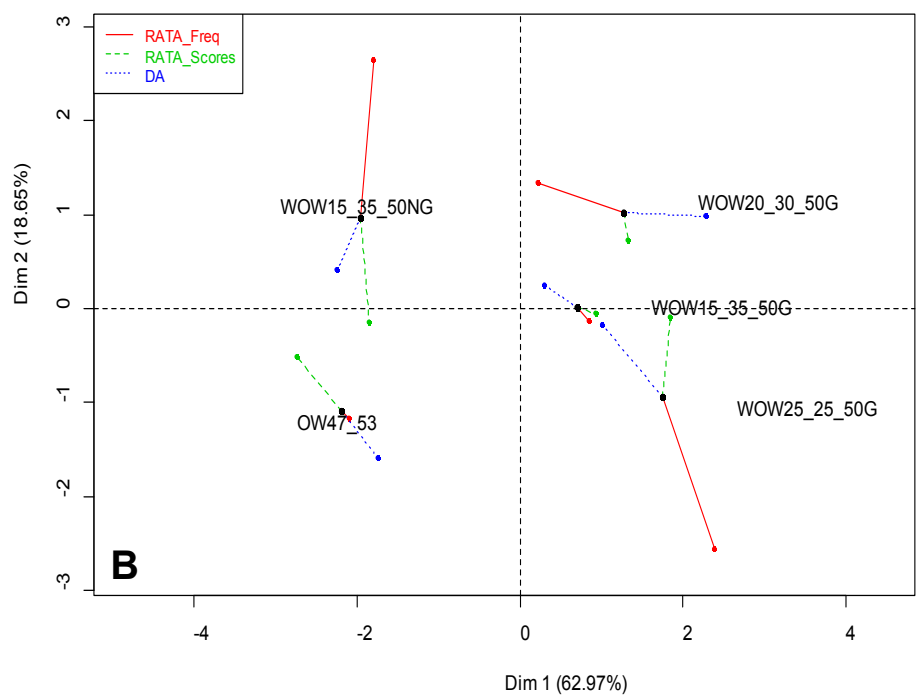

Figure 6.2 Consensus MFA sample space (first two components) with superimposed partial points from individual methods. (A) emulsion set with $30 \%$ dispersed phase, (B) emulsion set with $50 \%$ dispersed phase. 
Visual inspection of Figure $\mathbf{6 . 2}$ shows that in both MFAs, the first dimension mostly described product variation with regards to oil droplet properties: from full-fat emulsions and double emulsions with a low degree of fat reduction and non-gelled inner water phase on the left to double emulsions with a high degree of fat reduction and a gelled inner water phase on the right. In the MFA with the set of emulsions with $30 \%$ dispersed phase (Figure 6.2A), configuration similarity between all three methods was similar. In the MFA with the set of emulsions with $50 \%$ dispersed phase (Figure 6.2B), RATA intensity had more similar sample maps than RATA frequency when compared to DA. In this figure, the three data matrices correlated similarly with the first MFA component (31.8\% for RATA frequency, $36.5 \%$ for RATA intensity, and $31.7 \%$ for DA). The percentages refer to the contribution of individual groups to the MFA component, and differed slightly with regards to the second component $(63.1 \%$, $10.3 \%$ and $26.6 \%$, respectively).

\subsubsection{Comparison of individual sample configurations}

For completeness, also individual sample configurations produced by untrained (RATA as frequencies and intensities) and trained (DA) panellists were compared visually. As for the MFA, we compare the sample configurations based on the sensory terms that were used in both methodologies. Appendix $\mathbf{6 . 2}$ and $\mathbf{6 . 3}$ show sample configurations from RATA questions analyzed with frequency of term selection only (Appendix 6.2A and 6.3A), analyzed with RATA intensity (Appendix 6.2B and 6.3B) and DA (Appendix 6.2C and 6.3C) for the emulsion sets with 30 (Appendix 6.2) and $50 \%$ dispersed phase (Appendix 6.3). As also seen from the MFA configurations, RATA as intensities approach and DA resulted in closer configurations than RATA as frequencies approach and DA. In all cases, the first dimension mostly described variation with regards to oil droplet properties: from full-fat emulsions and double emulsions with a non-gelled inner water phase on the left to double emulsions with a gelled inner water phase on the right. In the correspondence analyses, emulsions with a gelled inner water phase were always closely associated with sensory terms like thick, creamy, and cohesive. PCAs of RATA intensities and DA showed that most terms were similarly associated with the same emulsions with both methodologies. Correspondence analyses differed from this observation mostly based on fat-related sensory terms (fatty (MF, AF), and sunflower oil $(T)$ ). It should be noted from these representations that the large confidence ellipses reflect the small perceptual differences of the product sets, also reflected in the data and analyses shown in Tables $\mathbf{6 . 3}$ and $\mathbf{6 . 4}$ and Appendix 6.1. However, precisely because samples are placed close to the center and the representation graphically maximizes the space, care should be taken when drawing conclusions from the size of the confidence ellipses since they might appear even larger. 


\subsubsection{3 $R_{v}$ coefficients}

Sample configurations between analysis approaches (RATA frequency and RATA intensity) and methodologies (RATA and DA) were also compared by calculating the $R_{v}$ coefficients for each set of emulsions based on the first two dimensions and eight terms that were used in both methodologies. For completeness, also the $R_{v}$ coefficients obtained with all terms are given in Table 6.5. The $R_{v}$ coefficients for sample configurations between the two approaches to analyse RATA data (identical terms only) were 0.70 for the emulsion set with $30 \%$ dispersed phase (but not significant) and 0.82 for the emulsion set with $50 \%$ dispersed phase (Table 6.5). Both RATA approaches resulted in similar sample configurations in comparison to those obtained from DA for the $30 \%$ set $\left(R_{\text {V(RATA frequency vs. DA,30\%) }}=0.88\right.$; and $R_{V(R A T A \text { intensity vs. DA,30\%) }}=$ $0.87)$, but for the $50 \%$ set these were lower and not significant $\left(R_{V(R A T A}\right.$ frequency vs. DA,50\%) $=$ 0.60 , and $R_{V(R A T A \text { intensity vs. DA,50\%) }}=0.82$ ). The high $R_{v}$ coefficients cannot be justified by the low number of products because also analysing all ten emulsions together resulted in high $R_{v}$ coefficients, as shown in Table 6.5. Reducing the number of analysed sensory terms did not influence the calculated $R_{v}$ coefficients much. Regarding the comparison of RATA intensity and DA, similar $R_{v}$ values have been previously regarded as indicators of good agreement between sample configurations [8, 34-37].

Table $6.5 \mathbf{R}_{v}$ coefficients for sample configurations and the associated $p$-values.

\begin{tabular}{llcccccc}
\hline & & \multicolumn{2}{c}{$\begin{array}{c}\text { RATA frequency - } \\
\text { RATA intensity }\end{array}$} & \multicolumn{2}{c}{$\begin{array}{c}\text { RATA frequency - } \\
\text { DA }\end{array}$} & \multicolumn{2}{c}{$\begin{array}{c}\text { RATA intensity - } \\
\text { DA }\end{array}$} \\
\cline { 3 - 8 } & & $\mathrm{R}_{\mathrm{V}}$ & $\mathrm{p}$-value & $\mathrm{R}_{\mathrm{V}}$ & $\mathrm{p}$-value & $\mathrm{R}_{\mathrm{V}}$ & $\mathrm{p}$-value \\
\hline Emulsions 30\% & all terms* & 0.915 & 0.017 & 0.844 & 0.025 & 0.819 & 0.042 \\
dispersed phase & identical terms only** & 0.702 & 0.175 & 0.884 & 0.017 & 0.865 & 0.042 \\
Emulsions 50\% & all terms* & 0.961 & 0.008 & 0.794 & 0.058 & 0.873 & 0.050 \\
dispersed phase & identical terms only** & 0.821 & 0.050 & 0.604 & 0.200 & 0.817 & 0.058 \\
& all terms* & 0.945 & 0.000 & 0.770 & 0.002 & 0.720 & 0.003 \\
All 10 emulsions & identical terms only** & 0.796 & 0.002 & 0.778 & 0.002 & 0.663 & 0.005 \\
\hline
\end{tabular}

* "all terms" = 20 terms for RATA frequency/intensity, and 9 terms for DA

** "identical terms only" $=8$ terms that were used in both studies

\subsubsection{Correspondence between use of RATA and DA scales}

As shown in Appendix 6.1, a large proportion of non-checked sensory terms was observed in the RATA data. To explore whether the RATA task was interpreted and used in a similar manner as the scale in DA, we compared the proportion of scores of the emulsions for all eight common terms in both methods. Table $\mathbf{6 . 6}$ shows the descriptive comparison of two exemplar samples (WOW 9-21-70 NG and WOW 15-35-50 NG). It can be seen that despite the RATA scale being labelled as an intensity scale, it did not necessarily correspond to the ratings provided by the DA. 

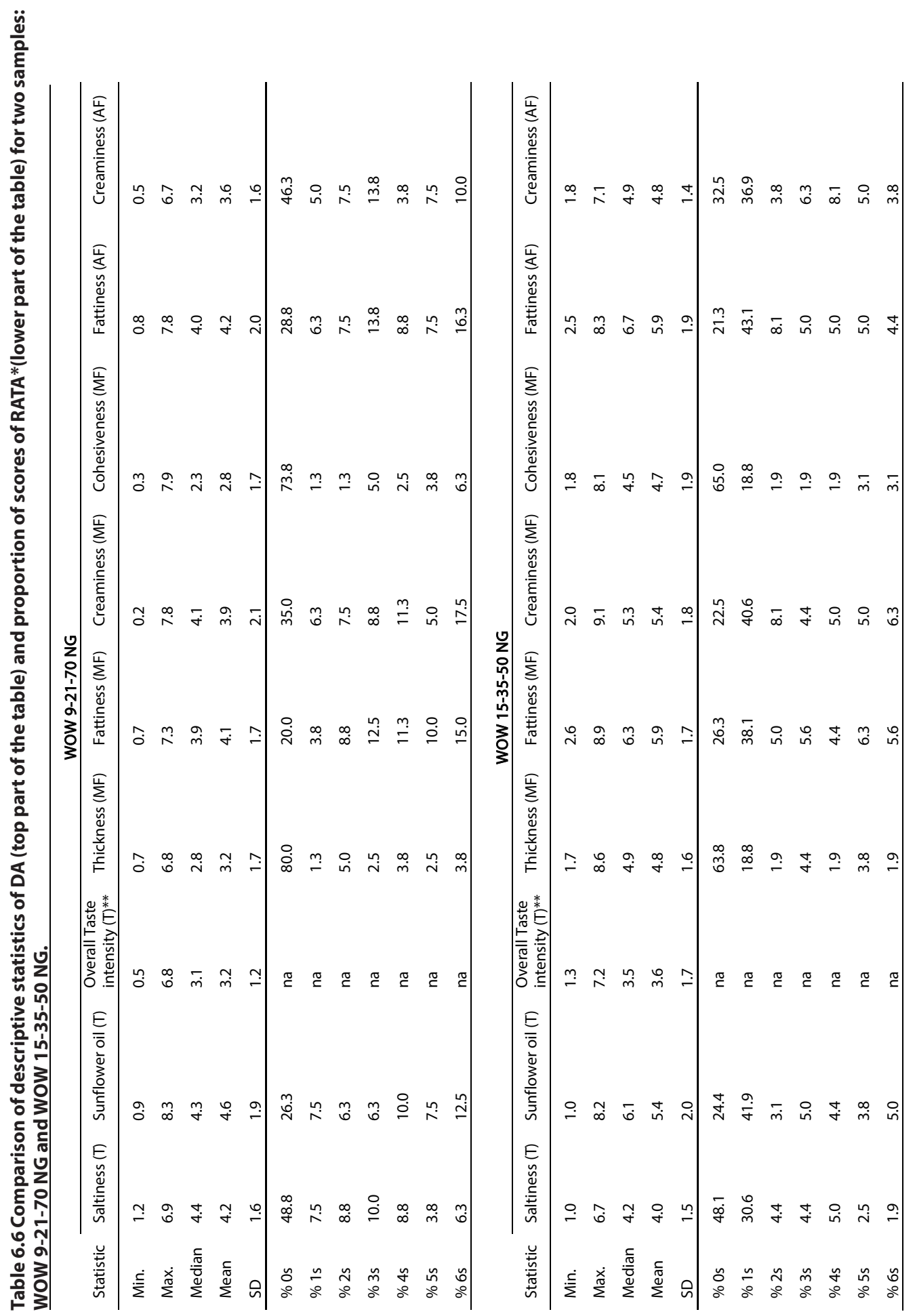
That is, a higher proportion of $0 \mathrm{~s}$ (or $1 \mathrm{~s}$ ) for a term did not always correspond with lower values in the DA scale. For instance, in case of the emulsion WOW 15-35-50 NG, the trained panel scored the thickness (MF) of the emulsion with 4.9 (median) and 4.8 (mean), while $64 \%$ of the untrained panel did not check this attribute and $19 \%$ of the panel gave it the lowest score (1). Only $17 \%$ of the participants gave it a score of 2 or higher. In case of the emulsion WOW 9-21-70 NG, the trained panel rated creaminess (MF) with 3.2 (median) and 3.6 (mean), while almost half of the untrained panel did not check the attribute. That means that RATA data should not be strictly interpreted as the 0 being the lower part of a $0-9$, at least with this type of samples. It seems that the scales were not equally interpreted and that there is a larger "psychological gap" between 0 and 1 than among the rest of the scale. In the general discussion this argument is further developed.

\subsection{General discussion and conclusions}

The aim of this study was to explore an alternative method to DA to investigate the sensory profiles of model emulsions and to discuss the applicability and caveats of different analyses approaches. The model emulsions used are a product category naïve consumers are not familiar with, and which exhibit subtle perceptual differences. As an alternative for DA performed with a trained panel, a two-step RATA approach was applied using an untrained panel. The two-step RATA approach comprised of first selecting the terms that applied to describe a sample, and secondly rating the perceived intensity on a 9-box scale when a term was selected. While DA data were analysed in a traditional way, RATA data were analysed with two approaches: Considering the frequencies of selection, or considering the rated intensities from 0 (unchecked) to 9 (max. possible score) of the sensory terms. Since the interpretation of methodological comparisons can be influenced by various decisions made when analysing sensory data, the next paragraphs will discuss some methodological decisions taken, as well as relevant points that have important implications to draw conclusions from the results.

Firstly, the RATA methodology included 21 terms, whereas only 9 terms were used in DA. The use of an extended list of sensory terms in the RATA study was considered useful for untrained participants to describe this type of products. For example, some sensory terms like thickness were split into two separate terms (thin, thick). Although sensory profiling by naive consumers was easier due to the longer list of terms, the extended list of sensory terms obstructed a direct comparison of methodologies. For that reason, to compare the discriminative ability between methodologies (RATA 
vs. DA), configuration similarity (MFA) and $R_{v}$ coefficients were evaluated based on the eight terms that were used in both methodologies.

Regarding sample discrimination, results from this study suggest that the use of the RATA data is slightly more powerful than treating RATA data as binary CATA data. As mentioned previously in section 6.3.1, several reasons for this finding can be considered: (i) Small perceptual differences in emulsions are easier to describe by rated intensities, (ii) the use of the 9-box scale facilitates to express the perceived intensities in sufficiently small steps, (iii) the addition of the familiarization session improves awareness and sensitivity for the subtle differences, and (iv) inclusion of 0s for non-checked sensory terms leads to an increase in significant differences between samples. Additional studies with samples with subtle perceptual differences are recommended to investigate the exact influence of a familiarization session.

The maps obtained from our data suggest that the RATA intensity approach led to a sample configuration closer to DA than did RATA frequency, although in general the three led to the same general conclusions.

The $R_{v}$ coefficients were lower when comparing the RATA frequency approach with RATA intensity and DA. It could possibly be due to data being richer in the RATA scores than in the binary CATA responses. The maps suggested as well that RATA intensity really carry additional (or different) information to discriminate products, since RATA frequency was the most different mostly for the $50 \%$ sample set. It should be noted that the multivariate analyses considered few products (5) and terms (8); this increases the ability to display all information in the first two dimensions, which are typically the most similar ones. We therefore suggest to investigate this issue with a larger number of products and terms to verify if then the maps of the three approaches differ more. Also, further investigations are required to determine whether using RATA data has superior properties than real CATA data obtained from a CATA study.

The results show that the results are sensitive to the statistical method used, especially in the way that non selected terms were included in the analysis. An important issue is whether for the RATA intensity data, non-checked sensory terms should be considered as 0 s or not and be included in the statistical analysis. In our analysis, we included 0 s when a term was not selected, despite the fact that the RATA method was a two-step procedure. In a follow-up enquiry most participants (76 \% of participants) indicated that they did not choose a term when it was not at all noticed, which justified this choice. The rest indicated that they did not choose a term when it was barely perceivable and not dominant. As in other studies, a non-selected term was 
therefore decided to be treated equivalent to a "not-perceived" label (intensity $=0$ ). Despite the large proportion of $0 \mathrm{~s}$ in the data set (violating normality assumptions), parametric analyses (ANOVAs) led to similar results compared to non-parametric (Friedman's) analyses. Although the parametric approach of the RATA data seemed to be valid enough, this does not imply that the scale, ranging from 0 to the maximum of the scale in case a term is checked, can be safely treated as continuous. It can be inferred from our data that the difference between a 0 and a 1 (not checking a term or checking its lowest scale level) was interpreted differently by consumers than the difference between the other points of the scale. The larger number of unchecked boxes (0s) for many terms suggests so. The instructions to "check all attributes that are required to describe the characteristics of the sample" might have led to this more 'dominant/striking' aspect of perception. Aside, the trained panel in the DA task had also deeper knowledge of the product set, which makes finding this consistency between the use of RATA and DA scales more challenging. Moreover, despite the scale being labeled as an intensity scale, it did not really correspond to the ratings provided by the DA. That is, higher proportion of 0 s for a term did not correspond with lower values in the DA scale despite participants reporting that a non-noticeable term was left unchecked. This warrants further research on how consumers actually interpret RATA scales. It seems that RATA is interpreted more as a relative measurement of the perception rather than as an absolute one (as in an intensity scale). From this study, it is therefore not really possible to determine how untrained participants used the "not applicable" in the RATA task, despite obtaining their own reports, since the data itself shows that they use this first step to identify attributes that were particular in that sample rather than to indicate whether they were perceived at all. Therefore, more cautious conclusions should be drawn from simply high $\mathrm{R}_{\mathrm{v}}$ coefficients and similar sample configurations.

The acceptability of $0 \mathrm{~s}$ when a term is non-ticked remains a topic of discussion, especially for products in other contexts, e.g. when products are familiar, or when differences are large. It is recommended in future studies to investigate participants' strategies and interpretation of the scale when performing a RATA task. For example, it is worth exploring whether participants tick a term as soon as they perceive it or only if its intensity is beyond their (personal) threshold. In the latter case, assigning Os to non-ticks would underestimate ratings and influence results by increasing subtle differences just by construction of data. Moreover, we recommend to define "applicability" well in future RATA studies. 
In conclusion, with this two-step RATA approach we show that naive participants are able to provide similar results compared to those obtained by DA with trained panellists, even with samples belonging to an unfamiliar product category with subtle differences. Analysing RATA data based on rated intensities showed a slight superior discriminative ability compared to analysing based on frequency of selection only and similar discriminative ability compared to DA. We conclude that RATA provides a good alternative to the time- and resource-intensive descriptive sensory analysis for these types of samples. However, as mentioned before, important issues should be taken into account when comparing methodologies. These include the consideration of non-checked terms as 0 s in RATA intensity, the influence of mathematical computation on results, and the number of samples and terms considered for methodological comparison. Taken together, our results suggest that researchers have to be more cautious about implications of methodological decisions.

\section{Acknowledgements}

This work was supported by the European Union Seventh Framework Programme (FP7/2007-2013), grant agreement nr. 289397 (TeRiFiQ project). The authors also thank the two reviewers for their constructive comments which significantly contributed to improving the quality of the publication. 


\section{References}

1. Stone, H. and J.L. Sidel, Sensory Evaluation Practices. 3rd ed. 2004, Orlando, FL: Academic Press.

2. Varela, P. and G. Ares, Novel Techniques in Sensory Characterization and Consumer Profiling. Vol. 1. 2014: CRC Press.

3. Varela, P. and G. Ares, Sensory profiling, the blurred line between sensory and consumer science. A review of novel methods for product characterization. Food Research International, 2012. 48: p. 893-908.

4. Adams, J., et al. Advantages and uses of check-all-that-apply response compared to traditional scaling of attributes for salty snacks. in 7th Pangborn sensory science symposium. 2007. Minneapolis, USA

5. Ares, G., et al., Comparison of two sensory profiling techniques based on consumer perception. 2010. 21: p. 417-426.

6. Jaeger, S.R., et al., Check-all-that-apply (CATA) responses elicited by consumers: Within-assessor reproducibility and stability of sensory product characterizations. Food Quality and Preference, 2013. 30: p. 56-67.

7. Ares, G., et al., Evaluation of a rating-based variant of check-all-that-apply questions: Rate-allthat-apply (RATA). Food Quality and Preference, 2014. 36: p. 87-95.

8. Ares, G., et al., Comparison of sensory product profiles generated by trained assessors and consumers using CATA questions: Four case studies with complex and/or similar samples. Food Quality and Preference, 2015. 45: p. 75-86.

9. Reinbach, H.C., et al., Comparison of three sensory profiling methods based on consumer perception: CATA, CATA with intensity and Napping. 2014. 32: p. 160-166.

10. Giacalone, D. and P.I. Hedelund, Rate-all-that-apply (RATA) with semi-trained assessors: An investigation of the method reproducibility at assessor-, attribute- and panel-level. Food Quality and Preference, 2016. 51: p. 65-71.

11. Meyners, M., S.R. Jaeger, and G. Ares, On the analysis of Rate-All-That-Apply (RATA) data. Food Quality and Preference, 2016. 49: p. 1-10.

12. Chung, C., et al., Reduced Fat Food Emulsions: Physicochemical, Sensory, and Biological Aspects. Critical Reviews in Food Science and Nutrition, 2015: p. 650-685.

13. Akhtar, M., et al., Factors affecting the perception of creaminess of oil-in-water emulsions. Food Hydrocolloids, 2005. 19: p. 521-526.

14. Benjamins, J., et al., Partial coalescence as a tool to control sensory perception of emulsions. Food Hydrocolloids, 2009. 23: p. 102-115.

15. Balcaen, M., et al., Influence of internal water phase gelation on the shear- and osmotic sensitivity of W/O/W-type double emulsions. Food Hydrocolloids, 2016. 58: p. 356-363.

16. Stone, H., et al., Sensory evaluation by Quantitative Descriptive Analysis. Food Technology, 1974. 28 : p. 24.

17. Jones, L.V., D.R. Peryam, and L.L. Thurstone, Development of a scale for measuring soldiers' food preferences. Journal of Food Science, 1955. 20: p. 512-520.

18. Jaeger, S.R. and A.V. Cardello, Direct and indirect hedonic scaling methods: A comparison of the labeled affective magnitude (LAM) scale and best-worst scaling. Food Quality and Preference, 2009. 20: p. 249-258.

19. Chojnicka-Paszun, A., H.H.J. de Jongh, and C.G. de Kruif, Sensory perception and lubrication properties of milk: Influence of fat content. International Dairy Journal, 2012. 26: p. 15-22.

20. van Aken, G.A., M.H. Vingerhoeds, and R.A. de Wijk, Textural perception of liquid emulsions: Role of oil content, oil viscosity and emulsion viscosity. Food Hydrocolloids, 2011. 25: p. 789-796.

21. van Aken, G.A., et al., Aspects of sensory perception of food emulsions thickened by polysaccharides, in Gums and Stabilisers for the food industry 13, P.A. Williams and G.O. Phillips, Editors. 2006, Cambridge: Royal Society of Chemistry. p. 449-456.

22. Manoukian, E.B., Mathematical nonparametric statistics. 1986, New York: Gordon \& Breach.

23. Escofier, B. and J. Pagès, Multiple factor analysis (AFMULT package). Computational Statistics \& Data Analysis, 1994. 18: p. 121-140.

24. Robert, P. and Y. Escoufier, A unifying tool for linear multivariate statistical methods: The RV coefficient. Applied Statistics, 1976. 25: p. 257-265.

25. Josse, J., J. Pagès, and F. Husson, Testing the significance of the RV coefficient. Computational Statistics \& Data Analysis, 2008. 53: p. 82-91.

26. Husson, F., et al., FactoMineR: multivariate exploratory data analysis and data mining with $R$. $\mathrm{R}$ package version 1.24, 2013. 
27. Lê, S., J. Josse, and F. Husson, FactoMineR: An $R$ package for multivariate analysis. Journal of Statistical Software, 2008. 25: p. 1-18.

28. Ares, G. and S.R. Jaeger, Examination of sensory product characterization bias when checkall-that-apply (CATA) questions are used concurrently with hedonic assessments. Food Quality and Preference, 2015. 40: p. 199-208.

29. Li, B., J.E. Hayes, and G.R. Ziegler, Interpreting consumer preferences: Physicohedonic and psychohedonic models yield different information in a coffee-flavored dairy beverage. Food Quality and Preference, 2014. 36: p. 27-32.

30. Husson, F., S. Le Dien, and J. Pagès, Which value can be granted to sensory profiles given by consumers? Methodology and results. Food Quality and Preference, 2001. 12: p. 291-296.

31. Hayes, J.E., B.S. Sullivan, and V.B. Duffy, Explaining variability in sodium intake through oral sensory phenotype, salt sensation and liking. Physiology \& Behavior, 2010. 100: p. 369-380.

32. Ares, G., F. Bruzzone, and A.N.A. Gimenez, Is a consumer panel able to reliably evaluate the texture of dairy desserts using unstructured intensity scales? Evaluation of global and individual performance. Journal of Sensory Studies, 2011. 26: p. 363-370.

33. Worch, T., S. Lê, and P. Punter, How reliable are the consumers? Comparison of sensory profiles from consumers and experts. Food Quality and Preference, 2010. 21: p. 309-318.

34. Lelièvre, M., et al., What is the validity of the sorting task for describing beers? A study using trained and untrained assessors. Food Quality and Preference, 2008. 19: p. 697-703.

35. Kennedy, J., Evaluation of replicated projective mapping of granola bars. Journal of Sensory Studies, 2010. 25: p. 672-684.

36. Lawless, H.T. and S. Glatter, Consistency of multidimensional scaling models derived from odor sorting. Journal of Sensory Studies, 1990. 5: p. 217-230.

37. Abdi, H., et al., Analyzing assessors and products in sorting tasks: DISTATIS, theory and applications. Food Quality and Preference, 2007. 18: p. 627-640. 
Appendix

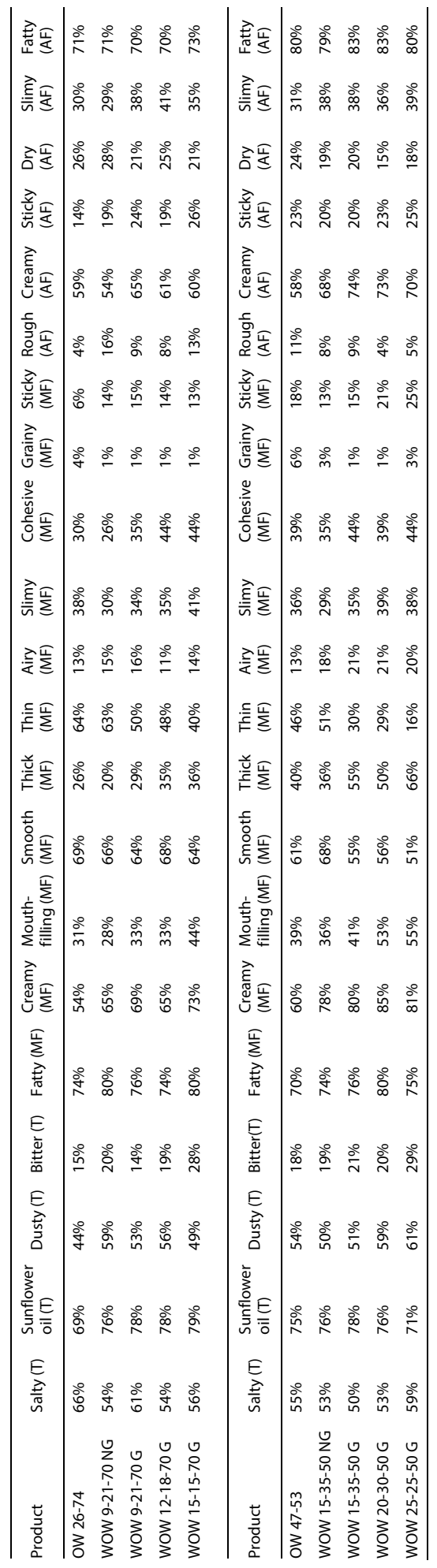


Appendix 6.2 Correspondence Analysis on RATA frequency data (A), Principal Component Analysis on RATA intensity data (B), and Principal Component Analysis on DA data (C), all for emulsions with $30 \%$ dispersed oil phase. PCA are split into loading plot (left) and product map including confidence ellipses (right). CA and PCA from the RATA question were based on those terms that were also used in DA.
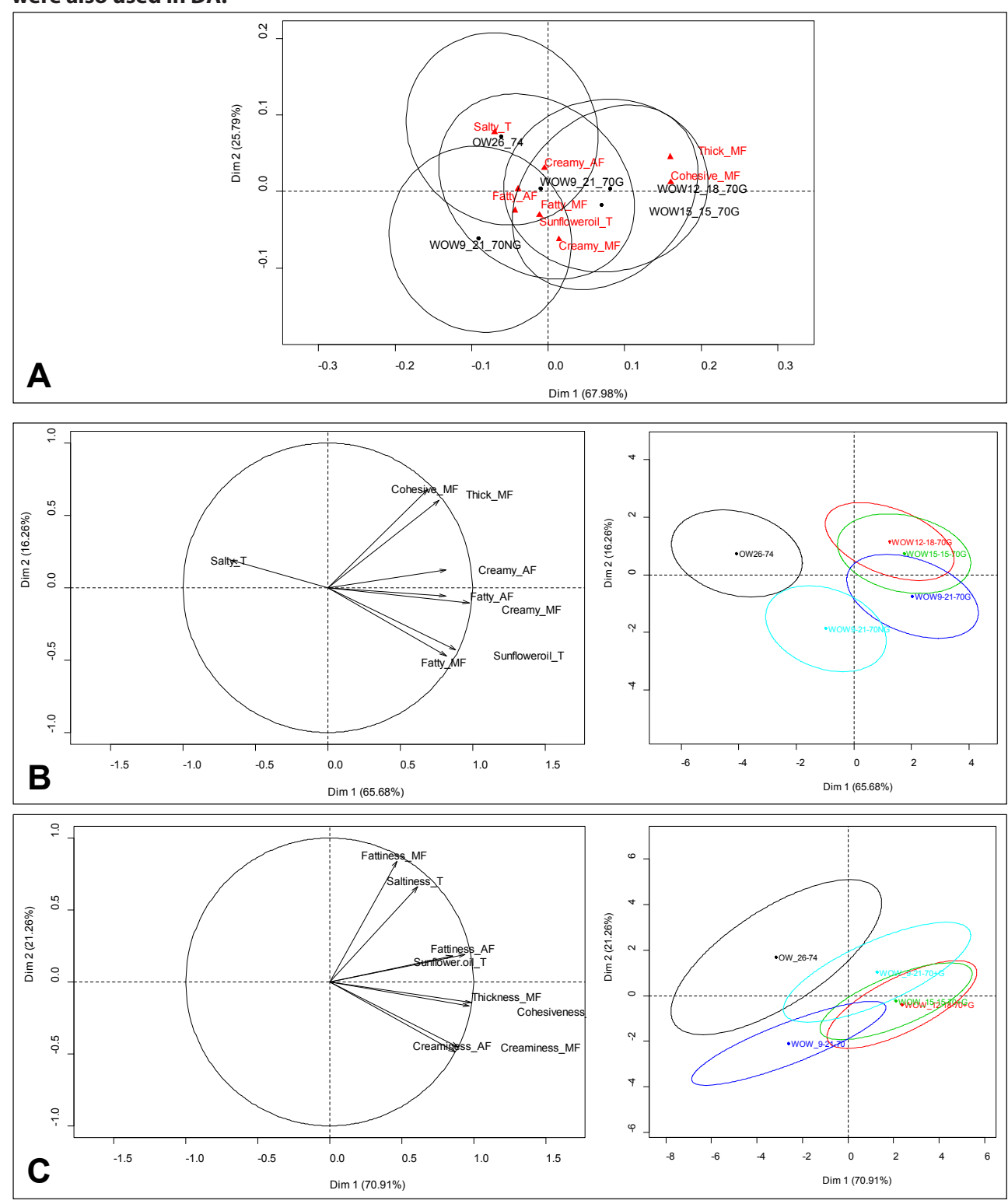
Appendix 6.3 Correspondence Analysis on RATA frequency data (A), Principal Component Analysis on RATA intensity data (B), and Principal Component Analysis on DA data (C), all for emulsions with $50 \%$ dispersed oil phase. PCA are split into loading plot (left) and product map including confidence ellipses (right). CA and PCA from the RATA question were based on those terms that were also used in DA.

A
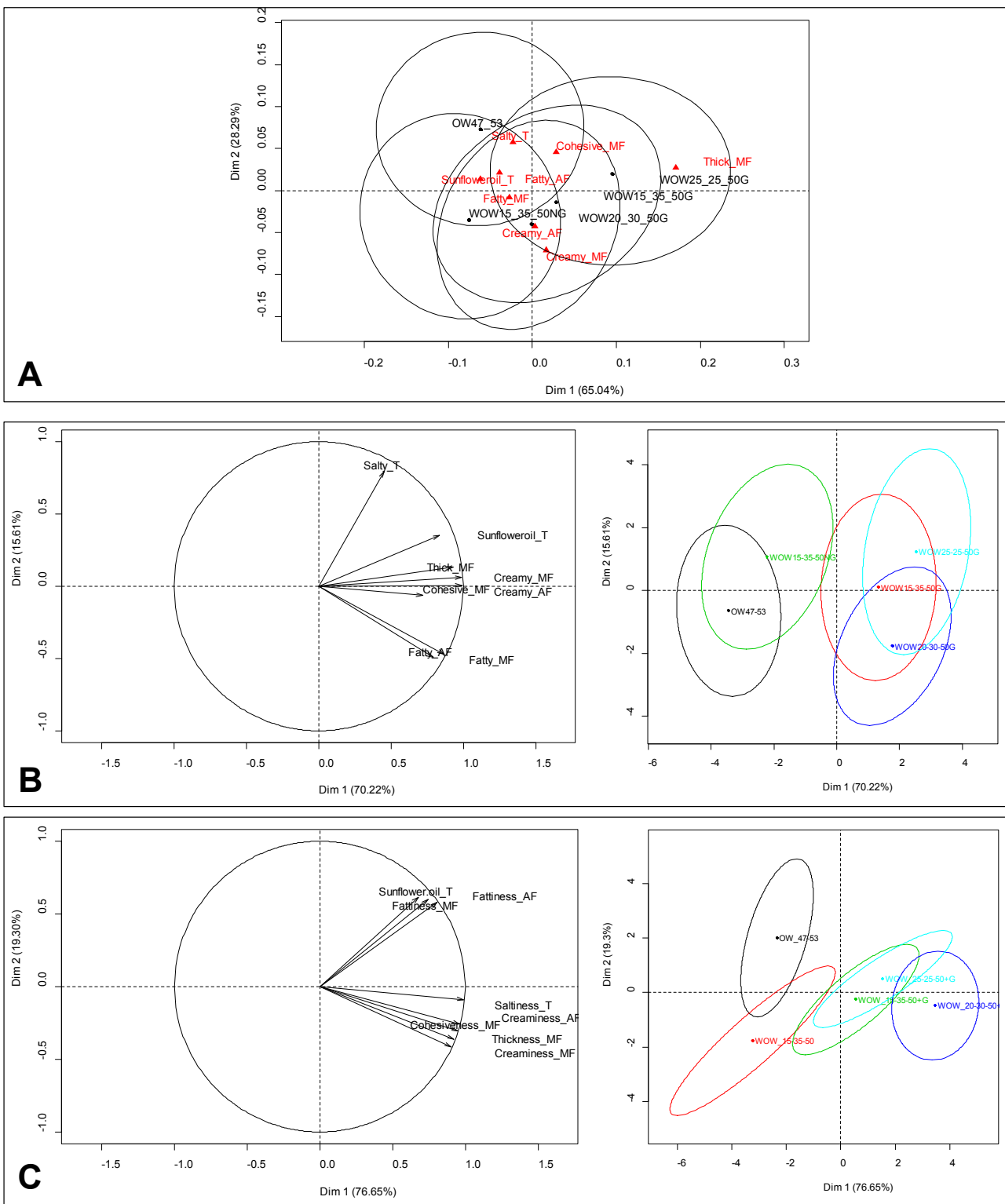


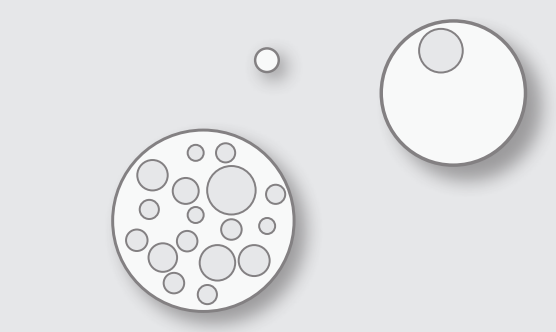

\section{Chapter 7}

General discussion
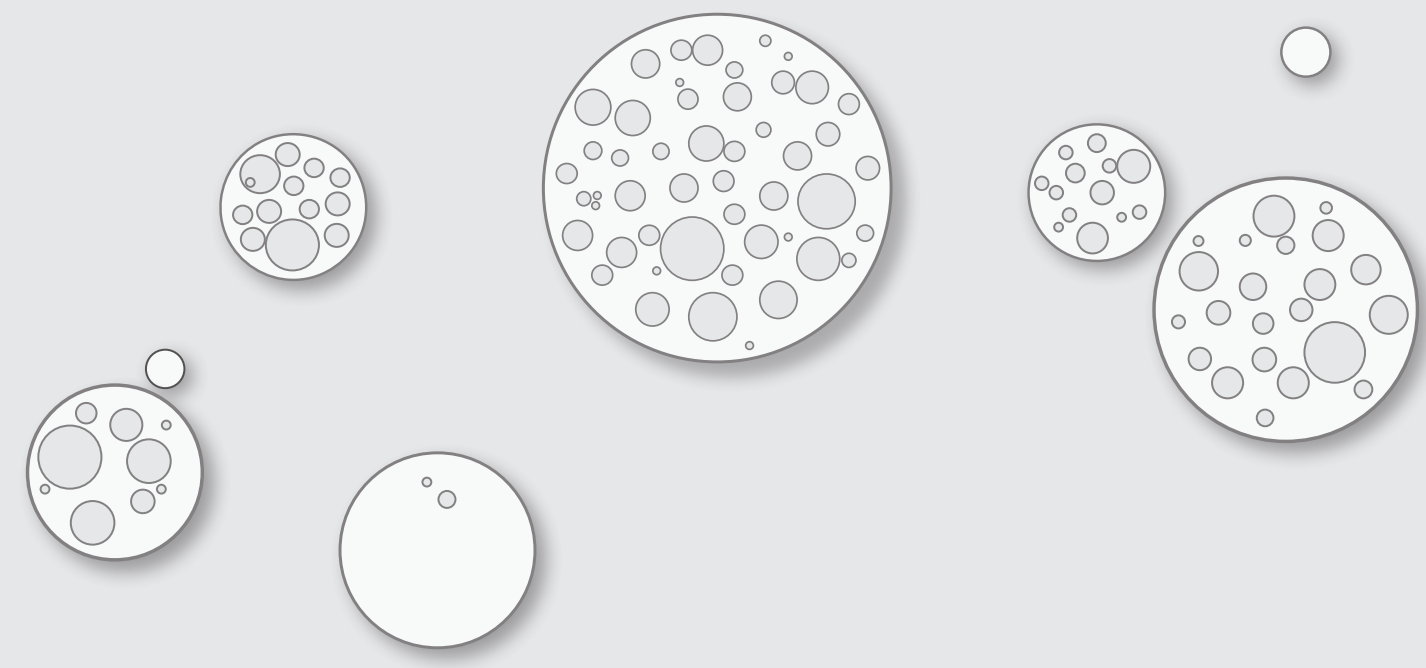


\subsection{Objective and main findings}

Fats and oils are high-caloric ingredients that contribute to the energy-density of foods. One approach to reduce the fat content in foods, the focus of this thesis, is the use of double $\left(w_{1} / o / w_{2}\right)$ emulsions as fat replacers. The aim of this thesis was to better understand the influence of composition and structure of double $\left(w_{1} / 0 / w_{2}\right)$ emulsions on physicochemical properties, stability against processing conditions and sensory perception. The main findings related to the design of double $\left(w_{1} / 0 / w_{2}\right)$ emulsions, their stability, lubrication properties and sensory perception are summarized in Figure 7.1.

In chapter 2, the effect of gelation and hence mechanical properties of the inner aqueous phase $w_{1}$ on double $\left(w_{1} / o / w_{2}\right)$ emulsion stability was studied. Yields were significantly increased by gelling the inner aqueous phase $w_{1}$ with gelatin and WPI, and stability against storage, heat and high shear differed depending on the mechanical properties of the gelled $w_{1}$ droplets.

In chapter 3, the composition and physicochemical properties of the oil-water $\left(\mathrm{o} / \mathrm{w}_{2}\right)$ interface and outer water phase $w_{2}$ were modified by varying the type of hydrophilic emulsifier and thickener. In addition to the results obtained in chapter $\mathbf{2}$, it was found that not only the mechanical properties of the inner aqueous phase, but also the interfacial and bulk properties of $w_{2}$ influence oil droplet size and yield of the inner $w_{1}$ phase of double emulsions.

In chapter 4, double emulsions varying in the level of fat reduction were designed to investigate the effect of emulsion composition on lubrication properties. Friction was dominated by the adsorption of PGPR on the hydrophobic tribo-surfaces at low entrainment speeds, and by the level of fat reduction at intermediate entrainments. Particularly at higher levels of fat reduction, increased friction coefficients were probably linked to the presence of gelled particles expelled to the outer water phase $w_{2^{\prime}}$ and to the decreased deformability of the dispersed $\left(w_{1} / 0\right)$ droplets.

In chapter 5, the sensory perception of double emulsions, varying in fat reduction $(0-50 \%)$ was studied by means of Descriptive sensory Analysis (DA) with a trained panel. The replacement of oil by small water droplets did not decrease the intensity of fat-related attributes. For higher fat reduction levels, the intensity of certain positive fat-related attributes (creaminess, thickness, cohesiveness) even increased, while fattiness perception was not affected. This demonstrated that replacement of oil by $w_{1}$ in double emulsions is a strategy to reduce fat content while maintaining or enhancing sensory perception of fat-related attributes. 


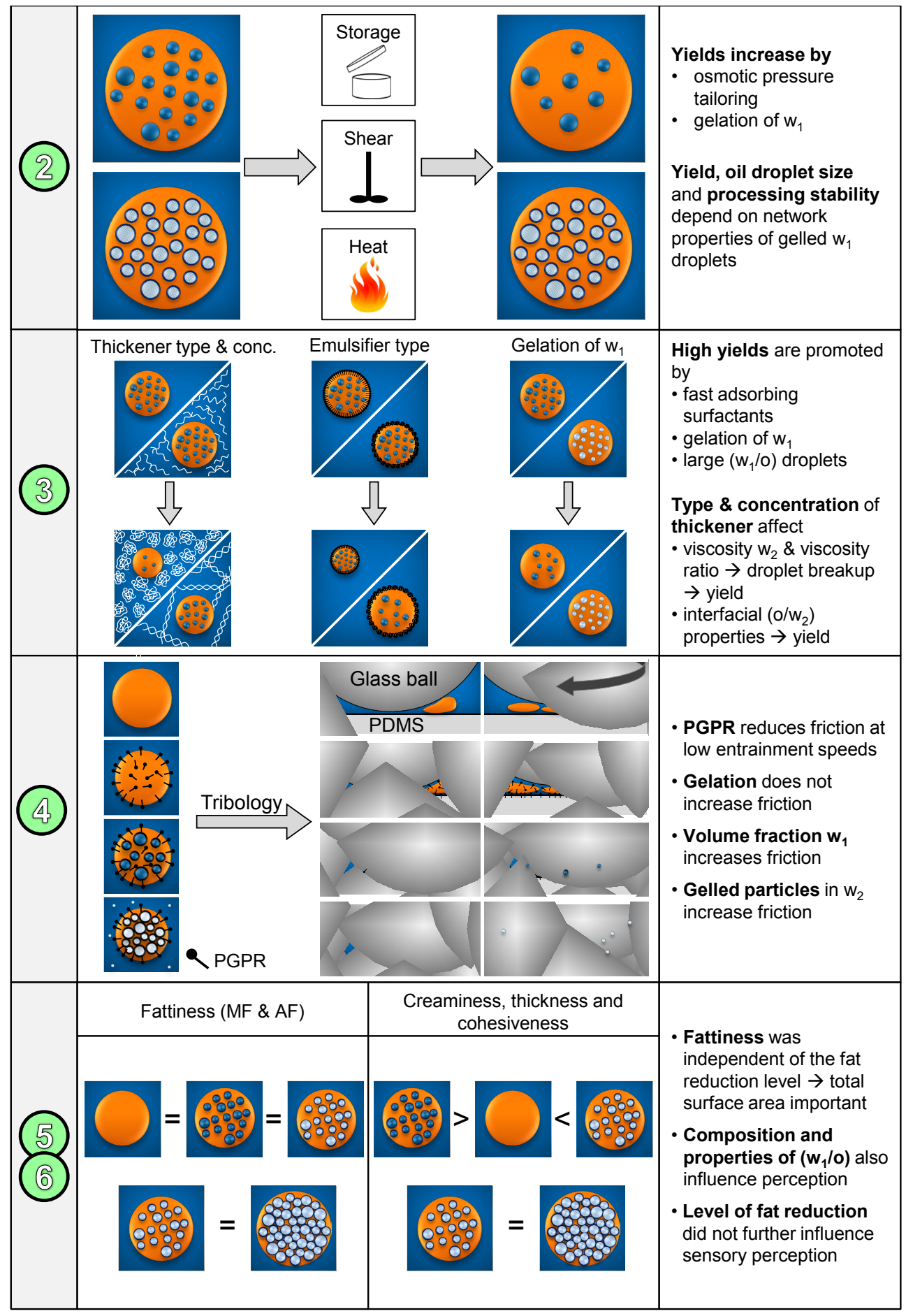

Figure 7.1 Schematic overview of main findings. Numbers indicate chapters in this thesis. 
Next to a trained panel performing Descriptive sensory Analysis (chapter 5), an alternative sensory methodology with untrained panellists (Rate-All-That-Apply (RATA)) was used in chapter 6. The comparison between the two methods is particularly interesting for the development of novel sensory techniques since most methodological studies in that field are usually carried out with (commercial) samples with clear differences in their sensory characteristics. Untrained participants performing RATA were able to provide similar descriptions to those obtained by trained participants performing DA. It was also highlighted that methodological decisions can have substantial implications on the conclusions drawn from the results. In the following sections, the implications of the findings are discussed with respect to the requirements of double $\left(w_{1} / o / w_{2}\right)$ emulsions to be considered a promising approach to reduce fat content in emulsions and emulsion-based foods. These requirements are:

1. high levels of fat reduction

2. stability against conditions encountered during processing and storage

3. mouthfeel and fat-related sensory perception similar to that of full-fat equivalents

\subsection{Emulsion design to maximize yield of $w_{1}$}

To apply double $\left(\mathrm{w}_{1} / \mathrm{o} / \mathrm{w}_{2}\right)$ emulsions as fat replacers, it is most important to achieve and maintain high levels of fat reduction during preparation and further processing. The level of fat reduction is expressed as the amount of inner aqueous droplets partially replacing oil. The amount of inner aqueous droplets can change over time for two reasons:

1. Diffusion of water molecules between the inner $w_{1}$ and outer $w_{2}$ aqueous phase

2. Coalescence of inner $w_{1}$ with outer $w_{2}$ aqueous phase

To employ double emulsions as fat replacers, it is essential to minimize water losses of the inner aqueous phase. The following subsections discuss approaches to achieve and maintain high levels of fat reduction.

\subsubsection{Reduce diffusion of water from $w_{1}$ to $w_{2}$}

It was shown in chapter 2, that already a small imbalance in osmotic pressure has detrimental effects on yield of $w_{1}$ phase. Even though diffusion of water from $w_{1}$ to $\mathrm{w}_{2}$ can be reduced by gelation of the $\mathrm{w}_{1}$ droplets, careful adjustment of the osmotic 
pressure gradient between the inner and outer aqueous phase is essential for the design of double $\left(\mathrm{w}_{1} / \mathrm{o} / \mathrm{w}_{2}\right)$ emulsions. It was shown in chapter $\mathbf{2}$, that by proper osmotic pressure tailoring, the yield and the stability can be improved. In real food products, however, this adjustment may be difficult to achieve since real foods usually contain a substantial amount of sugars, salts, and other compounds in the outer aqueous phase, influencing the osmotic pressure gradient. Very high concentrations of $\mathrm{NaCl}$ or other solutes in the inner $\mathrm{w}_{1}$ phase would be necessary to create the required gradient to reduce diffusion of water from $w_{1}$ to $w_{2}$. Practical limitations to determine the osmotic pressure of a real food product might additionally obstruct the adjustment of the required osmotic pressure gradient. Hence, design of double $\left(w_{1} / o / w_{2}\right)$ emulsions by osmotic pressure balancing alone is probably not sufficient to obtain stable double emulsions in real food products.

An osmotic pressure gradient towards the inner aqueous phase will lead to swelling of the inner aqueous droplets by water transfer from the outer phase into the inner aqueous droplets. The swelling leads to an increase in the dispersed $\left(w_{1} / 0\right)$ fraction, and could be used as a strategy to increase the level of fat reduction [1, 2]. However, upon swelling of the inner droplets, there is a risk that the dispersed $\left(w_{1} / 0\right)$ droplets swell to such extent that the $w_{1}$ droplets coalesce with $w_{2}[3,4]$. Osmotic swelling is therefore considered an interesting approach to increase the level of fat reduction in emulsions and emulsion-based foods yet difficult to apply in real foods.

\subsubsection{Minimize coalescence between $w_{1}$ and $w_{2}$}

Coalescence of inner $w_{1}$ droplets with the outer $w_{2}$ phase is a second reason, besides diffusion, for a decrease in yield. To achieve high yields and consequently high levels of fat reduction, it is crucial to minimize coalescence between the inner $w_{1}$ and outer $\mathrm{W}_{2}$ aqueous phase. Coalescence is affected by several factors:

1. Properties of the $\left(\mathrm{o} / \mathrm{w}_{2}\right)$ interface

2. Balance of viscous and interfacial stresses exerted at the $\left(\mathrm{o} / \mathrm{w}_{2}\right)$ interface during droplet breakup

3. Mechanical properties of the inner $w_{1}$ droplets

Firstly, coalescence of the inner $w_{1}$ with the outer $w_{2}$ aqueous phase can be minimized through modulation of the $\left(\mathrm{o} / \mathrm{w}_{2}\right)$ interfacial properties. Fast adsorption of low-molecular weight surfactants at the $\left(\mathrm{o} / \mathrm{w}_{2}\right)$ interface during the second emulsification step was found to reduce the $w_{1}-w_{2}$ coalescence at the newly created surfactant-stabilized interface (chapter 3 ). Loss of $w_{1}$ can be further minimized 
when competition of hydrophilic emulsifiers with other surface-active ingredients in the continuous phase is limited (chapter 3). Further, it has been shown that the hydrophobic emulsifier PGPR is also present at the $\left(0 / w_{2}\right)$ interface $[5,6]$, interacting with hydrophilic emulsifiers, weakening the interface. A weakening of the $\left(0 / w_{2}\right)$ interfacial film increases the probability of $w_{1}-w_{2}$ coalescence, and must therefore be minimized by careful selection of ingredients with limited adsorption at the $\left(\mathrm{o} / \mathrm{w}_{2}\right)$ interface.

Secondly, if viscous stresses exerted at the $\left(\mathrm{o} / \mathrm{w}_{2}\right)$ interface (altered by modifying the bulk viscosity and thereby changing the viscosity ratio between the dispersed $\left(w_{1} / 0\right)$ and continuous $w_{2}$ phase) dominate the interfacial stresses, dispersed $\left(w_{1} / 0\right)$ droplets become unstable and break up (Chapter 3$)$. This breakup of $\left(w_{1} / 0\right)$ droplets into smaller droplets leads to an increased probability of $w_{1}-w_{2}$ coalescence due to an increase of contact area between the two interfaces. It was found throughout all chapters that $w_{1}-w_{2}$ coalescence was generally reduced with increased dispersed $\left(w_{1} / 0\right)$ droplet size. The exact relation between the two parameters was shown to depend on the composition (mechanical properties of $w_{1}$ droplets, $\left(0 / w_{2}\right)$ interfacial characteristics) of the emulsion and the viscosity ratio of the dispersed $\left(w_{1} / 0\right)$ and continuous $\left(w_{2}\right)$ phase. This relation was also previously shown by others. Schuch and co-workers showed that yields of $w_{1}$ phase of double emulsions are related to oil droplet size, regardless of the homogenization device used for the second emulsification step [7]. The finding that yield-oil droplet size relationship depends on emulsion composition (chapter $\mathbf{2}$ and $\mathbf{3}$ ) shows the complexity of understanding double emulsion characteristics.

Thirdly, chapters $\mathbf{2}$ - $\mathbf{5}$ showed that coalescence can be minimized by gelling the inner aqueous $w_{1}$ droplets. To maximize yields through gelation of $w_{1}$, one has to contemplate on the type of gelling agent and concentration used, since network properties of the gelled droplets influence their mechanical properties, which in turn influence yields. Chapter 2 showed that yields increased by up to $20 \%$ with increasing hardness (fracture stress) and stiffness (modulus) of gelled $w_{1}$ droplets. Decreasing the deformability of the gelled $w_{1}$ droplets increases the required energy to expel those droplets through the oil-water $\left(0 / w_{2}\right)$ interface. This insight goes beyond current knowledge to increase yields through gelation of the inner aqueous droplets. However, a more systematic study is required to better understand the relation between $w_{1}$ mechanical properties, yield, and other emulsion characteristics such as oil droplet size. 


\subsubsection{From high yields to high levels of fat reduction}

Based on the previous section, the following parameters promote high yields:

- Osmotic pressure - Laplace pressure balance

- Gelation of inner aqueous phase $w_{1}$

- Large dispersed $\left(\mathrm{w}_{1} / 0\right)$ droplets

- Fast diffusion/adsorption rate of surfactant to the $\left(0 / w_{2}\right)$ interface

All these parameters must be considered in the emulsion design to decrease $w_{1}-w_{2}$ coalescence. To achieve high yields, all of these requirements should be met. Not meeting one of those requirements can have detrimental effects on yield.

To not only minimize losses of $w_{1}$ through $w_{1}-w_{2}$ coalescence, but also increase the achievable level of fat reduction, gelation of the inner aqueous phase is crucial (chapters 4 - 6). The highest level of fat reduction obtained without gelation of $\mathrm{w}_{1}$ droplets is about $30 \%$ only (chapters 4 - 6). This was also previously found by Schuch and co-workers [8]. For higher fractions of non-gelled $w_{1}$ droplets, more water droplets collide with the $\left(\mathrm{o} / \mathrm{w}_{2}\right)$ interface, leading to rupture of the oil film separating the two aqueous phases [8]. Additionally, the oil film separating the water droplets becomes thinner with increasing amounts of small water droplets, increasing the probability of film rupture $[9,10]$. In order to achieve fat reduction levels higher than $30 \%$, it is necessary to gel the inner aqueous droplets, since it increases the energy barrier to be overcome for $w_{1}-w_{2}$ coalescence. Only with gelation of $w_{1}$, fat reduction levels of up to $50 \%$ are possible.

Another possibility to achieve higher levels of fat reduction is not to gel the inner aqueous droplets but the oil droplets themselves. Solidification of the lipid by increasing the solid fat content of the oil (semi-solid fats) may hinder $w_{1}-w_{2^{\prime}}$ and also $\mathrm{w}_{1}-\mathrm{w}_{1}$ coalescence $[11,12]$.

\subsubsection{Methodological aspects regarding yield determination}

As shown in the sections above, the yield of double emulsions is an important property since it denotes the fat reduction level. As yield of inner aqueous phase $w_{1}$ is difficult to measure directly, numerous methods have been developed to measure the yield in an indirect way. Indirect techniques do not determine the amount of the inner $w_{1}$ phase, but instead measure the release of an encapsulated material. Indirect measurement techniques can be based on electroconductivity, photometry or rheometry. 
In the case of electroconductivity, electrolytes are dissolved in $w_{1}$ and their diffusion to the outer water phase is determined by measuring the (change in) conductivity in the outer $w_{2}$ phase [13-19]. Challenges related to this technique are linked to the influence of oil droplets on the conductivity [20], binding of electrolytes to hydrophilic emulsifiers [18], and the diffusion of electrolytes independent from the transport of water [21,22], leading to insensitivity of this measurement technique [23]. Moreover, electrolytes influence the osmotic pressure gradient to a large extent, which may lead to diffusion of water, as shown in chapter 2. When water diffuses from one aqueous phase to the other (especially from $w_{1}$ to $w_{2}$ ) without diffusion of the electrolytes, no changes in conductivity are measured. Therefore, yields determined by electroconductivity are not necessarily related to the amount of entrapped water.

For photometric measurements, molecules such as colouring substances, Vitamin $\mathrm{B}_{12^{\prime}}$ resveratrol or flavonoids are often encapsulated as markers in the inner aqueous $w_{1}$ phase and their release to the outer $w_{2}$ phase is determined [24-30]. To determine the amount of released marker, the outer aqueous $w_{2}$ phase has to be separated by centrifugation from the dispersed $\left(w_{1} / 0\right)$ phase. Centrifugation may induce additional release of inner $w_{1}$ droplets. Markers with low molecular weights or partly hydrophobic markers may diffuse through the oil. Further, it is possible that a large fraction of the released marker remains in the creamed layer after centrifugation and that yield of the marker cannot be measured accurately [28]. Especially when no calibration based on the recovery yield in $\left(\mathrm{o} / \mathrm{w}_{2}\right)$ emulsions is performed, an accurate measurement of the yield cannot be obtained [28]. Often high yields are reported based on photometric measurements while micrographs of the double emulsions show rather empty oil droplets [25-27, 30, 31].

Rheometry, in contrast to the previously discussed techniques, is not based on encapsulation of molecules but on changes in viscosity caused by a release of inner water droplets [23]. A release of $w_{1}$ decreases the amount of dispersed phase while increasing the amount of the continuous $w_{2}$ phase, and a subsequent decrease in viscosity can be observed. This correlation can be used to estimate the total dispersed phase content of a double emulsion. However, this approach is only valid when droplet sizes do not change, and when the inner $w_{1}$ phase has the same rheological properties as the outer water phase. Additionally, changes in viscosity will be obvious only for high dispersed phase fractions, and therefore this technique is not reliable for all types of emulsions.

Indirect methods to determine yield have in common that they are usually not very sensitive to changes in yield, tend to over- or underestimate yields and above all often 
do not directly correlate to the amount of encapsulated water [23]. These methods have therefore clear limitations with respect to the determination of entrapped water within the oil droplets. Therefore, a method to determine the amount of water directly is preferred. Currently, two methods exist to provide information about the amount of inner water content within the oil droplets of double emulsions. One of them is based on differential scanning calorimetry (DSC), which differentiates the inner $w_{1}$ and outer $w_{2}$ phase during freezing in a calorimeter due to subcooling of compartmentalized $w_{1}$ droplets [32]. This technique has only been used recently and is a direct technique to determine yields as the latent heat of freezing is directly related to the mass of the water. DSC has been shown to be sensitive to changes in yield and provides reliable information [7, 8, 23, 32-34]. Despite the clear advantage of the direct determination of the water content in the oil droplets, the disadvantage is that the measurements are based on very small sample sizes (7-12 mg). As only small sample sizes are included, it can be difficult to take a representative sample and multiple measurements are recommended to obtain an average value. In addition, it would not be possible to determine the amount of inner water droplets correctly if diffusion of water between the two aqueous phases takes place during measurements. Therefore, the sample has to be stable during the analysis time of about $25 \mathrm{~min}$.

The other direct method is based on low resolution pulsed field gradient nuclear magnetic resonance measurements (PFG-NMR) [35, 36]. PFG-NMR has been used to measure droplet size distributions [37] but only recently also for determination of yield [35,36]. However, reported yields of about 45 and $85 \%$ at dispersed $\left(w_{1} / 0\right)$ droplet sizes smaller than $6.5 \mu \mathrm{m}$, together with rather empty oil droplets shown in micrographs, give rise to doubts whether yields are overestimated. It was shown in chapters $\mathbf{2}$ and $\mathbf{3}$, but also by others [7], that yields are correlated to oil droplet size. Typically, yields are below $10 \%$ for oil droplet sizes below $10 \mu \mathrm{m}$ and therefore the yields reported by Balcaen and co-workers [35] contradict the observations in this thesis. Despite the suggested advantage of the direct determination of the water content in the oil droplets, disadvantages of PFG-NMR are that measurement times, just like for DSC measurements, are rather long (about $35 \mathrm{~min}$ ), and that creaming of large oil droplets might lead to overestimation. Moreover, additional corrections of $\mathrm{T}_{1}$ and $\mathrm{T}_{2}$ relaxation times are necessary if the compositions of the two aqueous phases differ [35].

While it has been shown by Schuch and co-workers [23] that DSC as a direct method is the preferred method compared to indirect methods to determine the amount of entrapped water, no comparison between PFG-NMR and DSC measurements to 
determine yields has been published so far. Therefore, it is proposed that DSC at this point is still the preferred measurement technique when the amount of inner water phase in double $\left(w_{1} / o / w_{2}\right)$ emulsions is to be determined.

\subsection{Stability of double emulsions}

\subsubsection{Stability against creaming}

As previously discussed, oil droplets need to be rather large to hold a substantial amount of small $w_{1}$ droplets. This has an effect on emulsion stability as large droplets considerably increase the creaming rate. To reduce creaming, the viscosity of the outer aqueous $\mathrm{w}_{2}$ phase can be increased. However, as shown in chapter 3 , this is not beneficial as an increase in outer water phase viscosity prior to emulsification facilitates oil droplet breakup, which leads to reduced oil droplet sizes and consequently lower yields. Additionally, as discussed in section 7.2.2, the addition of polysaccharides that are able to adsorb at the oil-water interface (or protein-residues thereof) influence interfacial $\left(\mathrm{o} / \mathrm{w}_{2}\right)$ properties, thereby leading to additional loss of inner water droplets. This contradicts the common belief that thickeners are beneficial for the stability of double $\left(w_{1} / o / w_{2}\right)$ emulsions since they avoid coalescence of dispersed droplets and reduce creaming.

With respect to yields of double emulsions, it should therefore be carefully evaluated which thickener is used and how. If a thickener is required in the application, the use of non-adsorbing polysaccharides like xanthan or carboxymethyl cellulose (cmc) is recommended. Preferably, a double $\left(\mathrm{w}_{1} / \mathrm{o} / \mathrm{w}_{2}\right)$ emulsion is prepared before a thickener is added to obtain large $\left(w_{1} / o\right)$ droplets. If it is not possible to add the thickener after emulsification to avoid extensive oil droplet breakup, thickeners that show strong shear-thinning behaviour are recommended. The decrease in viscosity at higher shear rates leads to a decrease in the viscous stresses at the interface and reduces droplet deformation. Oil droplet breakup during emulsification is limited and losses of $w_{1}$ reduced. However, the choice of a suitable thickener depends also on other product characteristics such as interactions with other ingredients, $\mathrm{pH}$ and ionic strength in the specific food application.

\subsubsection{Stability over time}

As discussed in the previous section, interfacial $\left(0 / w_{2}\right)$ properties are involved in $w_{1}-w_{2}$ coalescence. The choice of hydrophilic emulsifier may therefore have a significant effect on the yield over time. Even though low molecular weight surfactants adsorb 
fast at the newly created $\left(\mathrm{o} / \mathrm{w}_{2}\right)$ interface during emulsification, resulting in high initial yields (chapter 3), their poor emulsion-stabilizing properties are hypothesized to result in $w_{1}-w_{2}$ coalescence and loss of $w_{1}$ over time. Proteins as emulsifiers on the other hand form a strong interfacial film and may increase stability over time. For long term stability, proteins as emulsifiers may therefore be a better choice. However, proteinstabilized (double) emulsions are often prone to aggregation and denaturation especially upon heating, as was observed in chapter $\mathbf{2}$ and also reported by Ye [38].

\subsubsection{Stability against processing conditions}

To apply double emulsions in food products, they also have to be stable with regard to changes in the amount of inner aqueous $w_{1}$ phase against processing conditions such as heat and shear. Gelation of $\mathrm{w}_{1}$ increases processing stability (chapter 2 ), but the exact magnitude depends on the network properties of the gelled $w_{1}$ droplets. Yields increased upon heating of the double $\left(\mathrm{w}_{1} / \mathrm{o} / \mathrm{w}_{2}\right)$ emulsion when gelatin was used as a gelling agent, while such an increase was not observed when whey protein isolate (WPI) was used as a gelling agent. This is due to the thermo-reversible nature of the gelatin. In the case shear was applied, emulsions with WPI as a gelling agent showed a larger decrease in oil droplet size and subsequently yield than when gelatin was used as a gelling agent. This indicates that the effect of a heat or shear treatment may be very different depending on the type of gelling agent used. Depending on the processing conditions of a specific potential application, type and concentration of gelling agent therefore have to be carefully chosen.

\subsection{Fat-related sensory perception of double emulsions}

\subsubsection{Link between sensory perception and double emulsion design}

As potential fat replacers, double emulsions have to provide a similar mouthfeel and sensory perception as that of their full-fat equivalents. As shown in chapter $\mathbf{5}$ and $\mathbf{6}$, fattiness was maintained and other fat-related sensory attributes such as creaminess were enhanced for fat reductions up to $47 \%$. These results demonstrate from a sensory perspective the potential of double emulsions as fat replacers.

As the total oil droplet surface area of fat-reduced double emulsions was similar to that of their full-fat equivalents, in chapter $\mathbf{5}$ it was proposed that fattiness is mostly determined by the oil droplet surfaces in contact with oral surfaces. Creaminess perception, in contrast, also depends on the bulk properties (composition and deformability) of the dispersed $\left(w_{1} / 0\right)$ droplets. In chapter $\mathbf{5}$, only double emulsions 
with large oil droplet sizes were used and compared to single emulsions with a similar oil droplet size. However, common food emulsions contain oil droplets of sizes in the micrometre range. Apart from the fact that the production of double emulsion droplets of that size is practically challenging and might lead to a complete loss of inner dispersed $w_{1}$ droplets, it is difficult to predict how single $\left(o / w_{2}\right)$ emulsions (e.g. average droplet size of $1 \mu \mathrm{m}$ ) would differ in sensory properties compared to double emulsions (average droplet size about $50 \mu \mathrm{m}$ ) with a total oil droplet surface about 50 times smaller. The effect of oil droplet size in $\left(\mathrm{o} / \mathrm{w}_{2}\right)$ emulsions on sensory perception is discussed controversially in literature [39-42]. It is currently not known to which extent perception of emulsions with large droplets is different than emulsions with small droplets. Additionally, the deformability of the dispersed droplets would differ and also affect sensory perception.

Considering the apparent importance of the contact between the oil droplet surface and the oral surfaces, smaller oil droplet sizes may be beneficial to enhance fat-related attributes. In the case the oil droplet surface area is an important factor for fat-related sensory perception, high shear homogenization techniques such as microfluidization may be used as an alternative to the approach of using double $\left(w_{1} / 0 / w_{2}\right)$ emulsions. The idea of microfluidization lies in the production of oil droplets smaller than those commonly found in food emulsions. By producing smaller droplets the total oil droplet surface might be maintained while actually reducing the fat content of a product [43-45]. This approach might be more easily applicable and scalable than the use of double emulsions, but needs to be further investigated in the future.

\subsubsection{Relation between sensory perception and tribological properties of double emulsions}

It has been suggested that creaminess perception of single emulsions can be linked to lubrication behaviour [42, 46-49]. In the following paragraph, the tribological behaviour of double emulsions is qualitatively compared with the sensory properties. The relation between results from tribological measurements (chapter 4) and those obtained from the Descriptive sensory Analysis (DA; chapter 5) are discussed.

While no difference in fattiness perception was found between full-fat single $\left(\mathrm{o} / \mathrm{w}_{2}\right)$ and double $\left(\mathrm{w}_{1} / \mathrm{o} / \mathrm{w}_{2}\right)$ emulsions, creaminess perception was enhanced for the double emulsions. A difference between the full-fat single and fat-reduced double emulsions is the use of PGPR in the double emulsions, which could have an effect on the sensory perception. Indeed, a large decrease in friction coefficient of $\left(0 / w_{2}\right)$ emulsions was found at low entrainment speeds upon addition of PGPR. This could 
indicate that the presence of PGPR is mainly responsible for the enhanced creaminess perception. However, since a single emulsion with added PGPR was not included in the sensory study in chapter $\mathbf{5}$, the effect of PGPR on the sensory perception of fatrelated attributes is not known and should be investigated.

Gelation of the inner $w_{1}$ droplets affected neither sensory nor tribological properties, indicating that not gelation of inner $w_{1}$ droplets itself but increasing real $w_{1}$ fraction enhanced sensory perception. Due to the small size of the inner water droplets, the large Laplace pressure of the droplets was already sufficiently high that gelation probably did not lead to a further increase in firmness. In double emulsions with higher levels of fat reduction (40 and $50 \%$ ), the inner water droplets were gelled using gelatin. While these emulsions were perceived as creamier, thicker and more cohesive than the full-fat single $\left(\mathrm{o} / \mathrm{w}_{2}\right)$ emulsion (chapter 5), no further decrease of friction at low entrainment speeds was observed, but an increase in friction at intermediate entrainment speeds was found (chapter 4). It is hypothesized that friction during tribological measurements increased due to reduced deformability of the oil droplets as well as the presence of gelled particles in the outer water phase $w_{2}$. This observation did not correspond to the observed enhanced creaminess perception. This divergence may be explained by a number of reasons. First, during the sensory evaluation, emulsions are mixed with saliva in the mouth while no saliva was used during tribological experiments. It is known that saliva can have a large influence on the emulsion properties [50-53]. For example, it has been shown that saliva provides not only strong lubricating properties itself [54-56], but that saliva might also lead to flocculation and coalescence of oil droplets, thereby influencing sensory perception $[52,57]$. As friction measurements were performed without saliva, results may not represent the conditions experienced during oral processing. Secondly, hydrophobic PDMS probes and a glass ball were used for the friction measurements. It has been shown previously that the surface properties such as roughness, hydrophobicity and elasticity of the tribo-pair have a strong influence on the measured friction $[58,59]$. It is therefore possible that the materials used in our study did not match the oral surfaces well enough. Thirdly, a sample in mouth will warm up during oral processing close to body temperature depending on the amount of the sample, the initial sample temperature and the duration of the sample present in the mouth. This increase in temperature may lead to structural changes of the emulsion, such as melting of gelled particles in the outer water phase or increase of the deformability of oil droplets. Friction measurements in chapter 4 , however, were performed at $20^{\circ} \mathrm{C}$. Melting of gelled particles inside or outside the oil droplets could decrease friction. 
In an additional experiment, the effect of temperature on friction measurements of a double emulsion containing $30 \%$ dispersed $\left(w_{1} / 0\right)$ phase, in which $50 \%$ of the oil was replaced by small gelled aqueous droplets was investigated. As shown in Figure 7.2, friction decreased at all entrainment speeds when the emulsion was warmed up from $20^{\circ} \mathrm{C}$ to $37^{\circ} \mathrm{C}$ during the measurement, and even more at intermediate entrainment speeds when the temperature of the emulsion was adjusted to $37^{\circ} \mathrm{C}$ already prior to the friction measurement. This indicates the strong influence of temperature on lubrication behaviour of emulsions and may therefore provide a plausible explanation for the discrepancy between observed sensory perception and measured friction coefficients. For a complete (or improved) understanding and overview, the role of saliva and temperature also has to be taken into account for the tribology experiments.

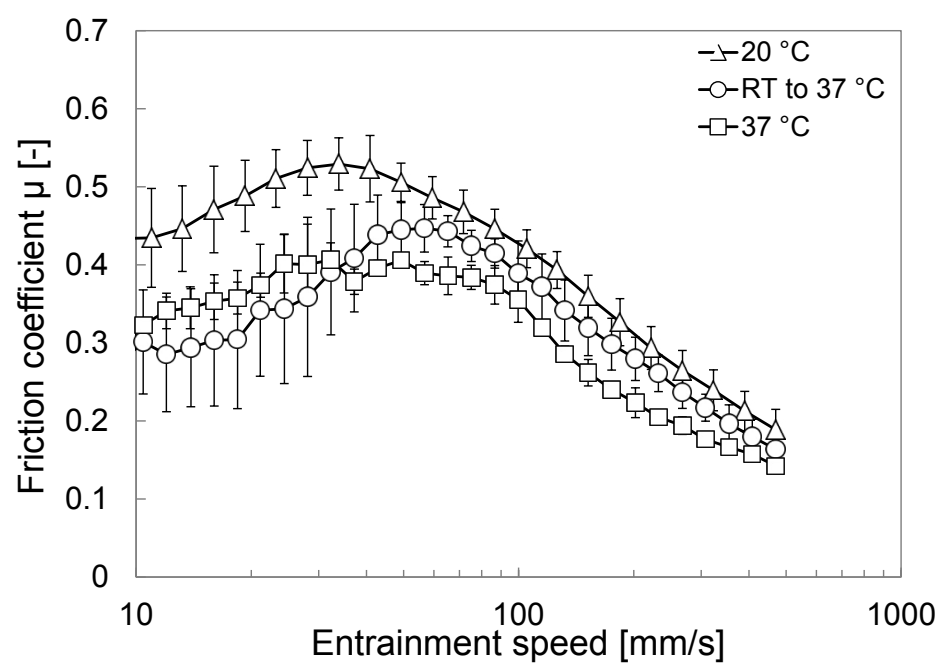

Figure 7.2 Friction coefficient of double emulsions WOW 15-15-70 G as a function of entrainment speed. The sample was measured either at room temperature $\left(20^{\circ} \mathrm{C}\right.$, triangles), warmed up during measurement from room temperature (RT) to $37^{\circ} \mathrm{C}$ (circles), or measured at $37^{\circ} \mathrm{C}$ (squares).

\subsection{Towards applications of double emulsions as fat replacers in foods}

This section shall critically discuss the achievements and progress with regard to the application of double emulsions, as well as highlight major remaining challenges from an application-perspective. While it was shown in this thesis that double $\left(w_{1} / 0 / w_{2}\right)$ emulsions can be designed with high fat reduction levels, have high stability against processing conditions and have very good sensory properties, their application as fat replacers was only investigated in a few studies [60-65]. The food types tested in those studies were reduced-fat cheese-like products, yoghurt and meat systems. Even though those studies found some physical or sensorial similarities between the 
full-fat and fat-reduced products and concluded that double emulsions can function as fat replacers in real food systems, these results should be taken with care. Basic requirements with regard to the emulsion design as presented in section 7.2.3 were not considered. Additionally, yields before or after application of the double emulsions in these (model) food products were not determined. Therefore, it is not clear whether double emulsions were stable enough to retain inner water droplets. In fact, when micrographs were reported, oil droplets were very small and appeared empty. This suggests that the results reported in literature are not based on welldesigned emulsions, and that limited amount of water droplets were present in the oil droplets. Currently, no reliable results for the application of double emulsions in foods have been reported.

As discussed throughout the previous sections, a food scientist or product technologist should be aware of the importance of the osmotic pressure gradient. In real food products, numerous ingredients can influence the osmotic pressure of the outer aqueous $\mathrm{w}_{2}$ phase. As this might lead to difficulties in adjusting the required osmotic pressure gradient, gelation of the inner water droplets is recommended to increase and maintain high yields. In chapters 4, 5 and $\mathbf{6}$, gelatin was used to gel the inner water droplets, which were used to replace up to $50 \%$ of oil, but also other types of gelling agents can be used. Taking into account the fact that network properties can be carefully designed and used to increase yield (chapter 2), this opens avenues to use alternative proteins as gelling agents. Alternatives to gelatin and WPI as gelling agents are particularly relevant for certain consumer groups, such as vegetarians and vegans, or certain product characteristics, such as kosher and halal. The requirements for the exact composition of inner water droplets depend on the type of food product, processing conditions, ease of application, and requirements regarding stability. The choice of hydrophilic emulsifier should further fit to the product with respect to emulsifying and stabilizing properties at the environmental conditions of the outer water phase (such as $\mathrm{pH}$ and ionic strength). Even though low molecular weight surfactants adsorb quickly at the $\left(\mathrm{o} / \mathrm{w}_{2}\right)$ interface, thereby increasing yields, proteins are considered more suitable at stabilizing emulsions for long-term stability. Moreover, to limit $w_{1}-w_{2}$ coalescence, the viscosity of the outer water and the shear rate during the second emulsification step should be kept as low as possible.

To stabilize the small inner $w_{1}$ droplets, a hydrophobic emulsifier is required. Polyglycerol polyricinoleate (PGPR) is the most common hydrophobic emulsifier used in double $\left(\mathrm{w}_{1} / \mathrm{o} / \mathrm{w}_{2}\right)$ emulsions. To our best knowledge, no other hydrophobic surfactant has similar emulsifying and stabilizing properties. However, the application 
of PGPR is limited by the European regulation 1129/2011. Current legislation indicates some constraints in the application of PGPR for double emulsions with regard to its maximum concentration, but also to the type of product it can be used in. The regulation permits the use of PGPR in certain spreadable fats, dressings, cocoa and chocolate products, as well as cocoa-based confectionery. Maximum dosages of PGPR in the final product vary between $4-5 \mathrm{~g} / \mathrm{kg}$ food product and acceptable daily intakes (ADI) of $7.5 \mathrm{mg} / \mathrm{kg}$ body weight should not be exceeded. If the double emulsions in this thesis were used directly as a real food product, they would already exceed the maximum PGPR concentration in the final product 2 to 3 times $(8.4 \mathrm{~g} / \mathrm{kg}$ in $30 \%$-fat reduced double emulsions, and $13.5 \mathrm{~g} / \mathrm{kg}$ in the case of $50 \%$-fat reduced double emulsions). It is therefore one of the major challenges to at least reduce the amount of PGPR used to stabilize inner $w_{1}$ droplets. Wolf and co-workers investigated the preparation of $\left(w_{1} / 0\right)$ emulsions exclusively based on gelation of water droplets without using PGPR [66]. Primary $\left(w_{1} / 0\right)$ emulsion preparation was only possible for very small amounts ( $3 \%$ ) of $w_{1}$, as gelation could not be induced fast enough to avoid aggregation and coalescence of $w_{1}$ droplets. Therefore, alternatives for PGPR as an emulsifier are necessary. As mentioned previously, also the use of solid fats might reduce coalescence and might aid to reduce the concentration of PGPR.

Next to challenges regarding stability and legal aspects of double emulsion application, also the size of oil droplets presents a major concern with regard to the application of double emulsions in food products. Since the size of the oil droplets directly influences the yield of the inner $w_{1}$ droplets of double emulsions, dispersed $\left(w_{1} / o\right)$ droplets have to be sufficiently large to retain a substantial number of smaller inner water droplets. Low shear processes are therefore necessary to avoid extensive droplet breakup. However, typical emulsification equipment aims at extensive droplet breakup to produce a homogeneous product of small oil droplets that does not cream during its shelf life. In double emulsions, creaming of the large dispersed $\left(w_{1} / o\right)$ droplets will not be avoidable. Thickeners can potentially be used to reduce creaming, but as shown in chapter 3 , the increase in viscosity prior to emulsification will lead to increased oil droplet breakup and subsequently lower yields. If it is not possible to add the thickener after emulsification, the use of non-adsorbing thickeners with strong shear-thinning behaviour is suggested as discussed in section 7.3.1. However, one has to be aware that the choices of a thickener depend also on other product characteristics such as interactions with other ingredients, $\mathrm{pH}$ and ionic strength. If a thickener has to be added prior to emulsification, preparation conditions should be adapted in such a way (e.g. lower homogenization pressures) that oil droplet breakup is still limited. 
In conclusion, application of double emulsions as fat replacers will be very productand formulation-specific. From a food manufacturer perspective, application of double emulsions will at this moment remain a challenge as meticulous adjustments in the emulsion design and processing conditions are required. In addition, legal aspects with respect to the use of PGPR as well as costs related to additional processing steps to prepare double $\left(\mathrm{w}_{1} / \mathrm{o} / \mathrm{w}_{2}\right)$ emulsions have to be considered.

\subsection{Directions for future research}

In this thesis, the complex interplay between emulsion composition, physical properties, and sensory perception of double emulsions was demonstrated. In addition to future challenges elaborated in the previous sections, the outcomes of the current thesis also elicit some suggestions for research in related fields.

The reduction of fat has been commonly associated with changes in the release of flavours and flavour profiles [67-75]. As fat droplet concentration influences perception of volatile and non-volatile flavour compounds by altering their partitioning between oil, water and headspace [76, 77], it is important to understand how the reduction of fat by means of double $\left(w_{1} / o / w_{2}\right)$ emulsions influences the flavour partitioning, flavour release and perception. Understanding thereof would further help to also comprehend to which extent flavour release is related to the overall amount of fat or to the surface area in contact with oral surfaces.

Fat droplets in emulsions and emulsion-based foods do not only contribute to a pleasurable sensory experience during eating, but may also affect the physiological response related to satiation and satiety $[68,78]$. Studies have indicated that unstable (aggregating and creaming) emulsion droplets in the stomach increase the rate of gastric emptying, and subsequently decrease the satiety response compared to those droplets that are stable [78, 79]. In the small intestine, fats are broken down into free fatty acids and monoacylglycerides. These digestion products contribute to the stimulation of chemosensory receptors to trigger the release of hormones which have been suggested to be linked to satiation by suppression of appetite $[68,80]$. It is unknown how replacing oil droplets by $\left(w_{1} / 0\right)$ droplets and stability of double emulsions in the stomach affect these responses and whether the use of proteins as gelling agents in double emulsions might promote satiation and satiety. 


\subsection{Concluding remarks}

This thesis contributed to the understanding of complex relations between double emulsion design, physicochemical properties, emulsion stability and sensory perception of double $\left(w_{1} / o / w_{2}\right)$ emulsions. It was shown that stable double emulsions with high levels of fat reduction can be prepared. Main drivers to obtain high yields were osmotic pressure tailoring, gelation of inner aqueous droplets, and presence of large dispersed $\left(w_{1} / o\right)$ droplet sizes. With regard to hydrophilic emulsifiers, a fast-adsorbing yet a strong film-forming emulsifier is preferred. Modifying network properties of the gelled inner aqueous phase further enhances the level of fat reduction, and increases stability during processing conditions. The fact that fat-related sensory perception of fat-reduced double emulsions was maintained or enhanced in comparison to that of full-fat single $\left(\mathrm{o} / \mathrm{w}_{2}\right)$ emulsions demonstrates the potential of double emulsions as fat replacers. While it was shown that through careful emulsion design, double emulsions with high levels of fat reduction can be designed and fat-related sensory properties maintained, application in real food products remains challenging. 


\section{References}

1. Iqbal, S., et al., Controlling W/O/W multiple emulsion microstructure by osmotic swelling and internal protein gelation. Food Research International, 2013. 54: p. 1613-1620.

2. Delample, M., F. Da Silva, and F. Leal-Calderon, Osmotically driven gelation in double emulsions. Food Hydrocolloids, 2014. 38: p. 11-19.

3. Mezzenga, R., B.M. Folmer, and E. Hughes, Design of double emulsions by osmotic pressure tailoring. Langmuir, 2004. 20: p. 3574-3582.

4. Florence, A.T. and D. Whitehill, Some features of breakdown in water-in-oil-in-water multiple emulsions. Journal of Colloids and Interface Science, 1980. 79: p. 243-256.

5. Bahtz, J., et al., Quantification of Spontaneous W/O Emulsification and its Impact on the Swelling Kinetics of Multiple W/O/W Emulsions. Langmuir, 2016. 32: p. 5787-5795.

6. Gülseren, I. and M. Corredig, Interactions at the interface between hydrophobic and hydrophilic emulsifiers: Polyglycerol polyricinoleate (PGPR) and milk proteins, studied by drop shape tensiometry. Food Hydrocolloids, 2012. 29: p. 193-198.

7. Schuch, A., J. Wrenger, and H.P. Schuchmann, Production of W/O/W double emulsions. Part II: Influence of emulsification device on release of water by coalescence. Colloids and Surfaces A: Physicochemical and Engineering Aspects, 2013. 461: p. 344-351

8. Schuch, A., et al., Production of W/O/W (water-in-oil-in-water) multiple emulsions: droplet breakup and release of water. Journal of Colloid and Interface Science, 2013. 402: p. 157-64.

9. Bahtz, J., et al., Decoupling of Mass Transport Mechanisms in the Stagewise Swelling of Multiple Emulsions. Langmuir, 2015. 31: p. 5265-5273.

10. Rosano, H.L., F.G. Gandolfo, and J.-D.P. Hidrot, Stability of $W_{1}-O-W_{2}$ multiple emulsions influence of ripening and interfacial interactions. Colloids and Surfaces A: Physicochemical and Engineering Aspects, 1998. 138: p. 109-121.

11. Frasch-Melnik, S., I.T. Norton, and F. Spyropoulos, Fat-crystal stabilised w/o emulsions for controlled salt release. Journal of Food Engineering, 2010. 98: p. 437-442.

12. Frasch-Melnik, S., F. Spyropoulos, and I.T. Norton, $W_{1} / O / W_{2}$ double emulsions stabilised by fat crystals--formulation, stability and salt release. Journal of Colloid and Interface Science, 2010. 350: p. 178-185.

13. Muguet, V., et al., W/O/W multiple emulsions submitted to a linear shear flow: Correlation between fragmentation and release. Journal of Colloid and Interface Science, 1999. 218: p. 335-337.

14. de Cindio, B., G. Grasso, and D. Cacace, Water-in-oil-in-water double emulsions for food applications: yield analysis and rheological properties. Food Hydrocolloids, 1991. 4: p. 339-353.

15. Schmidts, T., et al., Influence of hydrophilic surfactants on the properties of multiple W/O/W emulsions. Journal of Colloid and Interface Science, 2009. 338: p. 184-92.

16. Dragosavac, M.M., et al., Stirred cell membrane emulsification for multiple emulsions containing unrefined pumpkin seed oil with uniform droplet size. 2012. 392-393: p. 122-129.

17. Herzi, S., et al., Influence of the inner droplet fraction on the release rate profiles from multiple W/O/W emulsions. Colloids Surf., A, 2014. 441: p. 489-495.

18. Bonnet, M., et al., Release rate profiles of magnesium from multiple W/O/W emulsions. Food Hydrocolloids, 2009. 23: p. 92-101.

19. Pawlik, A.K. and I.T. Norton, Encapsulation stability of duplex emulsions prepared with SPG cross-flow membrane, SPG rotating membrane and rotor-stator techniques- $A$ comparison. Journal of Membrane Science, 2012. 415-416: p. 459-468.

20. Kostoglou, M., et al., Evolution of volume fractions and droplet sizes by analysis of electrical conductance curves during destabilization of oil-in-water emulsions. Journal of Colloid and Interface Science, 2010. 349: p. 408-416.

21. Cheng, J., et al., Transport of ions through the oil phase of $W_{(1)} / O / W_{(2)}$ double emulsions. Journal of Colloid and Interface Science, 2007. 305: p. 175-82.

22. Garti, N., Double emulsions - scope, limitations and new achievements. Colloids and Surfaces A: Physicochemical and Engineering Aspects, 1997. 123-124: p. 233-246.

23. Schuch, A., et al., Influence of the second emulsification step during production of W/O/W multiple emulsions: Comparison of different methods to determine encapsulation efficiency in W/O/W emulsions. The Canadian Journal of Chemical Engineering, 2014. 92: p. 203-209.

24. Fechner, A., et al., Stability and release properties of double-emulsions stabilised by caseinatedextran conjugates. Food Hydrocolloids, 2007. 21: p. 943-952. 
25. Akhtar, M., et al., Encapsulation of flavonoid in multiple emulsion using spinning disc reactor technology. Food Hydrocolloids, 2014. 34: p. 62-67.

26. Hemar, Y., et al., Encapsulation of Resveratrol Using Water-in-Oil-in-Water Double Emulsions. Food Biophysics, 2010. 5: p. 120-127.

27. Tamnak, S., et al., Encapsulation properties, release behavior and physicochemical characteristics of water-in-oil-in-water (W/O/W) emulsion stabilized with pectin-pea protein isolate conjugate and Tween 80. Food Hydrocolloids, 2016. 61: p. 599-608.

28. Regan, J.O. and D.M. Mulvihill, Water soluble inner aqueous phase markers as indicators of the encapsulation properties of water-in-oil-in-water emulsions stabilized with sodium caseinate. Food Hydrocolloids, 2009. 23: p. 2339-2345.

29. Perez-Moral, N., S. Watt, and P.J. Wilde, Comparitive Study of the Stability of Multiple Emulsions containing a gelled or aqueous internal phase. Food Hydrocolloids, 2014. 42: p. 215-222.

30. Surh, J., et al., Preparation and characterization of water/oil and water/oil/water emulsions containing biopolymer-gelled water droplets. Journal of Agricultural and Food Chemistry, 2007. 55: p. 175-184.

31. Perez-Moral, N., S. Watt, and P. Wilde, Comparative study of the stability of multiple emulsions containing a gelled or aqueous internal phase. Food Hydrocolloids, 2014. 42: p. 215-222.

32. Schuch, A., K. Köhler, and H.P. Schuchmann, Differential scanning calorimetry (DSC) in multiple W/O/W emulsions. Journal of Thermal Analysis and Calorimetry, 2013. 111: p. 1881-1890.

33. Schuch, A., L.G. Leal, and H.P. Schuchmann, Production of W/O/W double emulsions. Part I: Visual observation of deformation and breakup of double emulsion drops and coalescence of the inner droplets. Colloids and Surfaces A: Physicochemical and Engineering Aspects, 2013. 461: 336-343.

34. Schuch, A., et al., Observations on the influence of different biopolymers on coalescence of inner water droplets in W/O/W (water-in-oil-in-water) double emulsions. Colloids and Surfaces A: Physicochemical and Engineering Aspects, 2014. 475: p. 2-8.

35. Balcaen, M., et al., Influence of internal water phase gelation on the shear- and osmotic sensitivity of W/O/W-type double emulsions. Food Hydrocolloids, 2016. 58: p. 356-363.

36. Vermeir, L., et al., Influence of the NMR receiver gain on the enclosed water volume fraction of W/O/W double emulsions as determined by low-resolution NMR diffusometry and T2 relaxometry. Magnetic Resonance in Chemistry, 2015. 53: p. 309-313.

37. Wolf, F., et al., Preparation of $W_{1} / O / W_{2}$ emulsions and droplet size distribution measurements by pulsed-field gradient nuclear magnetic resonance (PFG-NMR) technique. European Journal of Lipid Science and Technology, 2009. 111: p. 730-742.

38. Ye, A., Surface protein composition and concentration of whey protein isolate-stabilized oil-inwater emulsions: Effect of heat treatment. Colloids Surf., B, 2010. 78: p. 24-29.

39. Vingerhoeds, M.H., et al., How emulsion composition and structure affect sensory perception of low-viscosity model emulsions. Food Hydrocolloids, 2008. 22: p. 631-646.

40. Kilcast, D. and S. Clegg, Sensory perception of creaminess and its relationship with food structure. Food Quality and Preference 2002. 13: p. 609-623.

41. Akhtar, M., et al., Factors affecting the perception of creaminess of oil-in-water emulsions. Food Hydrocolloids, 2005. 19: p. 521-526.

42. de Wijk, R.A. and J.F. Prinz, The role of friction in perceived oral texture. Food Quality and Preference, 2005. 16: p. 121-129.

43. Ciron, C.I.E., et al., Modifying the microstructure of low-fat yoghurt by microfluidisation of milk at different pressures to enhance rheological and sensory properties. Food Chemistry, 2012. 130: p. 510-519.

44. Ciron, C.I.E., et al., Comparison of the effects of high-pressure microfluidization and conventional homogenization of milk on particle size, water retention and texture of non-fat and low-fat yoghurts. International Dairy Journal, 2010. 20: p. 314-320.

45. Ciron, C.I.E., et al., Effect of microfluidization of heat-treated milk on rheology and sensory properties of reduced fat yoghurt. Food Hydrocolloids, 2011. 25: p. 1470-1476.

46. Chojnicka-Paszun, A., H.H.J. de Jongh, and C.G. de Kruif, Sensory perception and lubrication properties of milk: Influence of fat content. International Dairy Journal, 2012. 26: p. 15-22.

47. Dresselhuis, D.M., et al., Tribology of o/w Emulsions Under Mouth-like Conditions: Determinants of Friction. Food Biophysics, 2007. 2: p. 158-171.

48. Liu, K., et al., Evidence for ball-bearing mechanism of microparticulated whey protein as fat replacer in liquid and semi-solid multi-component model foods. Food Hydrocolloids, 2016. 52: p. 403-414.

49. Liu, K., et al., Effect of microparticulated whey protein on sensory properties of liquid and semisolid model foods. Food Hydrocolloids, 2016. 60: p. 186-198. 
50. Sarkar, A. and H. Singh, Oral Behaviour of Food Emulsions, in Food Oral Processing. 2012, Wiley-Blackwell. p. 111-137.

51. Dresselhuis, D.M., et al., The occurrence of in-mouth coalescence of emulsion droplets in relation to perception of fat. Food Hydrocolloids, 2008. 22: p. 1170-1183.

52. Vingerhoeds, M.H., et al., Emulsion flocculation induced by saliva and mucin. Food Hydrocolloids, 2005. 19: p. 915-922.

53. Silletti, E., et al., The role of electrostatics in saliva-induced emulsion flocculation. Food Hydrocolloids, 2007. 21: p. 596-606.

54. Bongaerts, J.H.H., D. Rossetti, and J.R. Stokes, The Lubricating Properties of Human Whole Saliva. Tribology Letters, 2007. 27: p. 277-287.

55. Vardhanabhuti, B., et al., Lubricating properties of human whole saliva as affected by $\beta$-lactoglobulin. Food Hydrocolloids, 2011. 25: p. 1499-1506.

56. Rossetti, D., et al., Astringency of tea catechins: More than an oral lubrication tactile percept. Food Hydrocolloids, 2009. 23: p. 1984-1992.

57. van Aken, G.A., M.H. Vingerhoeds, and R.A. de Wijk, Textural perception of liquid emulsions: Role of oil content, oil viscosity and emulsion viscosity. Food Hydrocolloids, 2011. 25: p. 789-796.

58. Dresselhuis, D.M., et al., Application of oral tissue in tribological measurements in an emulsion perception context. Food Hydrocolloids, 2008. 22: p. 323-335.

59. De Hoog, E.H.A., et al., Lubrication of Oral Surfaces by Food Emulsions: the Importance of Surface Characteristics. Journal of Food Science, 2006. 71: p. E337-E341.

60. Lobato-Calleros, C., et al., Reduced-fat white fresh cheese-like products obtained from $W_{1} / O / W_{2}$ multiple emulsions: Viscoelastic and high-resolution image analyses. Food Research International, 2006. 39: p. 678685.

61. Lobato-Calleros, C., et al., Structural and textural characteristics of reduced-fat cheese-like products made from $W_{1} / O / W_{2}$ emulsions and skim milk. LWT - Food Science and Technology, 2008. 41: p. 1847-1856.

62. Lobato-Calleros, C., et al., Microstructural and rheological properties of low-fat stirred yoghurts made with skim milk and multiple emulsions. Journal of Texture Studies, 2009. 40: p. 657-675.

63. Cofrades, S., et al., Preparation and impact of multiple (water-in-oil-in-water) emulsions in meat systems. Food Chemistry, 2013. 141: p. 338-46.

64. Cofrades, S., et al., Oxidative stability of meat systems made with W1/O/W2 emulsions prepared with hydroxytyrosol and chia oil as lipid phase. LWT - Food Science and Technology, 2014. 59: p. 941-947.

65. Freire, M., et al., Double emulsions to improve frankfurter lipid content: impact of perilla oil and pork backfat. Journal of the Science of Food and Agriculture, 2016. 96: p. 900-908.

66. Wolf, F., F. Gmoser, and H.P. Schuchmann, Stabilization of Water Droplets in an Oily Matrix Exclusively by Gel Formation. Chemical Engineering \& Technology, 2012. 35: p. 754-760.

67. Shamil, S., L.J. Wyeth, and D. Kilcast, Flavour release and perception in reduced-fat foods. Food Quality and Preference 1991. 3: p. 51-60.

68. McClements, D.J., Reduced-Fat Foods: The Complex Science of Developing Diet-Based Strategies for Tackling Overweight and Obesity. Advances in Nutrition: An International Review Journal, 2015. 6(3): p. 338S-352S.

69. Chung, C., et al., Reduced Fat Food Emulsions: Physicochemical, Sensory, and Biological Aspects. Critical Reviews in Food Science and Nutrition, 2016. 56: p. 650-685.

70. Frank, D., et al., Proton Transfer Reaction Mass Spectrometry and Time Intensity Perceptual Measurement of Flavor Release from Lipid Emulsions Using Trained Human Subjects. Journal of Agricultural and Food Chemistry, 2011. 59: p. 4891-4903.

71. Frank, D., et al., In vitro measurement of volatile release in model lipid emulsions using proton transfer reaction mass spectrometry. Journal of Agriculture and Food Chemistry, 2012. 60: p. 2264-2273.

72. Doyen, K., et al., Volatile Release from an Emulsion: Headspace and In-Mouth Studies. Journal of Agricultural and Food Chemistry, 2001. 49: p. 804-810.

73. Frank, D.C., et al., Effect of food matrix structure and composition on aroma release during oral processing using in vivo monitoring. Flavour and Fragrance Journal, 2012. 27: p. 433-444.

74. Malone, M.E., I.A.M. Appelqvist, and I.T. Norton, Oral behaviour of food hydrocolloids and emulsions. Part 2. Taste and aroma release. Food Hydrocolloids, 2003. 17: p. 775-784.

75. Malone, M.E. and I.A.M. Appelqvist, Gelled emulsion particles for the controlled release of lipophilic volatiles during eating. Journal of Controlled Release, 2003. 90: p. 227-241. 
76. Arancibia, C., et al., Flavor release and sensory characteristics of o/w emulsions. Influence of composition, microstructure and rheological behavior. Food Research International, 2011. 44: p. 1632-1641.

77. Chung, C. and D.J. McClements, Structure-function relationships in food emulsions: Improving food quality and sensory perception. Food Structure, 2013. 1: p. 106-126.

78. Marciani, L., et al., Effect of intragastric acid stability of fat emulsions on gastric emptying, plasma lipid profile and postprandial satiety. British Journal of Nutrition, 2008. 101: p. 919-928.

79. Marciani, L., et al., Enhancement of intragastric acid stability of a fat emulsion meal delays gastric emptying and increases cholecystokinin release and gallbladder contraction. American Journal of Physiology Gastrointestinal and Liver Physiology, 2007. 292: p. G1607-1613.

80. Stewart, J.E., C. Feinle-Bisset, and R.S.J. Keast, Fatty acid detection during food consumption and digestion: Associations with ingestive behavior and obesity. Progress in Lipid Research, 2011. 50: p. 225-233. 


$$
\frac{5}{0}
$$



Emulsions and emulsion-based foods, covering a wide range of commercial food products, usually contain a substantial amount of fat or oil. Fats and oils are high-caloric ingredients and contribute to the high energy-density of foods and consequently to the risk of obesity. To contribute to lowering the risk of obesity from a productperspective, there is a need for the development of fat-reduced food products. Reduction of fat content often leads to loss of desirable quality attributes such as texture and mouthfeel, which may be a result of a decrease in lubrication during oral processing. As common fat replacers have limited lubricating properties and can usually not replace all functions of emulsified fats or oils, there is a need to develop approaches to lower fat content, while maintaining sufficient contact between the oil and the oral cavity. One approach to lower fat content while maintaining a large contact area between the emulsified oil and oral surfaces is to design double $\left(w_{1} / o / w_{2}\right)$ emulsions. As an understanding of the relation between emulsion composition, fat reduction levels, stability and sensory perception is still limited, there is a need to understand these relationships to successfully use double emulsions as fat replacers in food products. The aim of this thesis was to better understand the influence of composition and structure of double $\left(w_{1} / o / w_{2}\right)$ emulsions on physicochemical properties, stability against processing conditions and sensory perception (chapter 1).

To design double emulsions with high yields, i.e. high amounts of inner aqueous phase remaining in the oil phase, and high stability over time and against processing conditions, the focus of chapter $\mathbf{2}$ was to understand how mechanical properties of the inner aqueous $w_{1}$ phase influence emulsion characteristics and stability. Mechanical properties of inner $w_{1}$ phase were varied by means of two gelling agents (gelatin and whey protein isolate (WPI)) at varying concentrations. In this study, the level of fat reduction (fraction of $\mathrm{w}_{1}$ inside the oil droplets) was $30 \%$ and whey protein isolate (WPI) was used as hydrophilic emulsifier in the outer water phase. Yield and oil droplet sizes were assessed directly after preparation, after storage (7 days at $20^{\circ} \mathrm{C}$ ), high shear conditions (5 min at $10000 \mathrm{rpm}$ in a high speed blender), and heat treatment ( $30 \mathrm{~min}$ at $97^{\circ} \mathrm{C}$ ). Initial yields significantly increased by $20 \%$ by gelling the inner aqueous phase $\mathrm{w}_{1}$ with gelatin and WPI, and double emulsions with a gelled inner aqueous phase were less sensitive towards an imbalance in osmotic pressure compared to those without gelling agents in $w_{1}$. Yields of double emulsions with or without gelling agents were stable over time with those with gelatin being slightly increasing due to slight swelling of the fine-stranded gel network. Stability against processing conditions differed depending on the mechanical properties of the gelled 
$w_{1}$ droplets. While initial yields generally increased with increasing fracture stress of the gelled droplets, double emulsions with gelled droplets with WPI as gelling agent were heat stable compared to those with gelatin, which increased in yield probably due to an increased osmotic pressure during heating due to the thermo-reversible nature of gelatin. Shear stability of double emulsions was improved when gelatin was used as a gelling agent, while droplets with a coarse WPI gel network led to a stronger decrease in oil droplet size and therefore yield upon shearing. Overall, yields significantly increased when the inner aqueous phase $\mathrm{w}_{1}$ was gelled with gelatin and WPI, and stability against storage, heat and high shear differed depending on the mechanical properties of the gelled $w_{1}$ droplets.

The second step was to investigate the effect of interfacial properties and bulk properties of the outer water phase $w_{2}$ on oil droplet breakup and double emulsion characteristics. In chapter 3 , the composition of the oil-water $\left(0 / w_{2}\right)$ interface and the outer aqueous $w_{2}$ phase was modified by varying the type of hydrophilic emulsifier (Tween 20, WPI, Na-caseinate) and the type of thickener (xanthan, highmethoxyl (HM) pectin). Viscosities of the outer aqueous $w_{2}$ phase were varied to obtain a broad range of viscosity ratios between the dispersed $\left(w_{1} / 0\right)$ phase and the outer water phase $w_{2}$. The resulting oil droplet sizes and yields of inner aqueous phases of the different double emulsions were determined. As in chapter $\mathbf{2}$, the level of fat reduction was $30 \%$. Fast diffusion of Tween 20, compared to that of WPI or $\mathrm{Na}$-caseinate, towards the $\left(\mathrm{o} / \mathrm{w}_{2}\right)$ interface led to smaller oil droplet sizes and higher yields of double emulsions. As in chapter 2, gelation of the inner water droplets increased the yield by $20 \%$. This was not only found for double emulsions stabilized by WPI, but also for those stabilized by Na-caseinate and Tween 20. Upon addition of thickeners, viscosity ratios decreased and facilitated droplet breakup, resulting in smaller oil droplet sizes and lower yields for all emulsions independent of the type of hydrophilic emulsifier. When pectin was used as a thickener, an additional decrease in yield was observed, indicating possible competition of pectin with hydrophilic emulsifiers at the $\left(\mathrm{o} / \mathrm{w}_{2}\right)$ interface. In addition to the results obtained in chapter $\mathbf{2}$, it was found that not only the mechanical properties of the inner aqueous $w_{1}$ phase, but also the $\left(0 / w_{2}\right)$ interfacial properties and the bulk properties of the outer $w_{2}$ phase influence oil droplet size and yield of the $w_{1}$ phase of double emulsions.

In chapter 4, the influence of the emulsion composition on lubrication properties was investigated, since lubrication properties are closely related to fat-related sensory perception. Double emulsions varying in the level of fat reduction $(0-50 \%)$ were designed to investigate the effect of emulsion composition on lubrication properties. 
Friction (the inverse of lubrication) was dominated by the adsorption of the lipophilic emulsifier polyglycerol polyricinoleate (PGPR) on the hydrophobic tribo-surfaces at low entrainment speeds, and by the level of fat reduction at intermediate entrainments. At intermediate entrainment speeds, friction behaviour of double emulsions for fat reduction levels up to $20 \%$ was comparable to that of full-fat single $\left(\mathrm{o} / \mathrm{w}_{2}\right)$ emulsions. For double emulsions with higher fractions of $w_{1}$ (corresponding to fat reductions of 40 and $50 \%$ ), friction was increased at intermediate entrainment speeds. This increase in friction can probably be related to the presence of gelled particles expelled from the oil droplets to the outer water phase $w_{2^{\prime}}$ as well as to the decreased deformability of the $\left(w_{1} / o\right)$ droplets.

As insights into fat-related sensory perception are crucial for the development of double emulsions as a fat replacement strategy, the sensory perception of double emulsions varying in their levels of fat reduction ( $0-50 \%)$ was studied by means of Descriptive sensory Analysis (DA) with a trained panel in chapter $\mathbf{5}$. As in chapter 3 and $\mathbf{4}$, the inner aqueous phase $w_{1}$ in some of the double emulsions was gelled, and emulsions were stabilized with WPI. The replacement of oil by small water droplets did not decrease the intensity of fat-related attributes. Fattiness perception was not affected by the level of fat reduction, proposing that it is dominated by the oil droplet surfaces in contact with oral surfaces. However, for other fat-related attributes, such as creaminess, thickness, and cohesiveness, the intensity was even increased when increasing the level of fat reduction with gelled droplets. These fat-related attributes seemed to depend more on the bulk properties (composition and deformability) of the dispersed $\left(w_{1} / o\right)$ droplets than on the total oil droplet surface area. Overall, this study demonstrated that double $\left(\mathrm{w}_{1} / \mathrm{o} / \mathrm{w}_{2}\right)$ emulsions can be used to reduce fat content of model emulsions while maintaining or enhancing sensory perception of fat-related attributes.

The aim of chapter 6 was to investigate whether an untrained panel using an adapted Rate-All-That-Apply (RATA) method provides similar sensory profiles of double emulsions as a trained panel using Descriptive sensory Analysis (DA). RATA as a novel sensory methodology with untrained panellists is an interesting alternative for the time and resource-intensive DA methodology. The comparison between the two methods using double emulsions is further interesting as most studies in that field are usually carried out with (commercial) samples with large differences in their sensory characteristics. It was found that untrained participants using RATA were able to provide similar sensory descriptions of the model emulsions with subtle perceptual differences to those obtained by DA with trained panellists. This demonstrates on one 
side that untrained participants can be used to profile sensory properties of model foods with subtle perceptual differences and on the other side that fat reductions of $47 \%$ can be achieved in double emulsions while maintaining the sensory properties. It was also highlighted, however, that methodological decisions can have substantial implications on the conclusions drawn from the results.

Chapter 7 summarised and integrated the findings of the different chapters of the thesis and discussed the significance of the results and implications towards applications of double emulsions as fat replacers. To apply double emulsions as fat replacers, they first have to be able to substantially reduce the level of fat/oil. The level of fat reduction is affected by the Osmotic - Laplace pressure balance, gelation of the inner aqueous $w_{1}$ phase, the oil droplet size and the type of hydrophilic emulsifier used. While all of these parameters must be considered to achieve high yields, gelation of the inner aqueous $\mathrm{w}_{1}$ phase was found most important to achieve fat reduction levels of up to $50 \%$. To use double emulsions as fat replacers, the choice of type and concentration of gelling agent and hydrophilic emulsifier depends on the application. To increase the applicability of double emulsions in a broad variety of foods, replacement of the lipophilic emulsifier PGPR may be required due to its legally restricted use. Creaming of the large dispersed $\left(w_{1} / 0\right)$ droplets also needs attention, and may require adaptations in the preparation process. Overall, it was highlighted that adjustments in the emulsion design and processing conditions are required to develop fat-reduced food products based on the use of double $\left(w_{1} / o / w_{2}\right)$ emulsions. In conclusion, significant progress was made in understanding the complex relations between double emulsion design, achievable levels of fat reduction, emulsion stability and sensory perception. The work presented in this thesis showed that through careful emulsion design, stable double emulsions with high levels of fat reduction can be designed while fat-related sensory properties are maintained, making double emulsions a promising approach for the development of fat-reduced food products. 

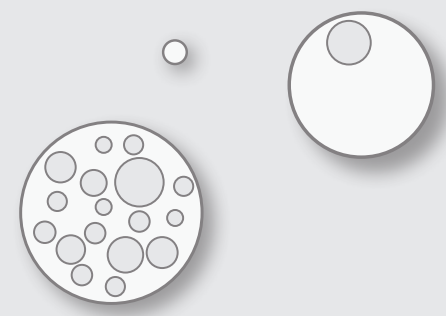

\section{Acknowledgements}
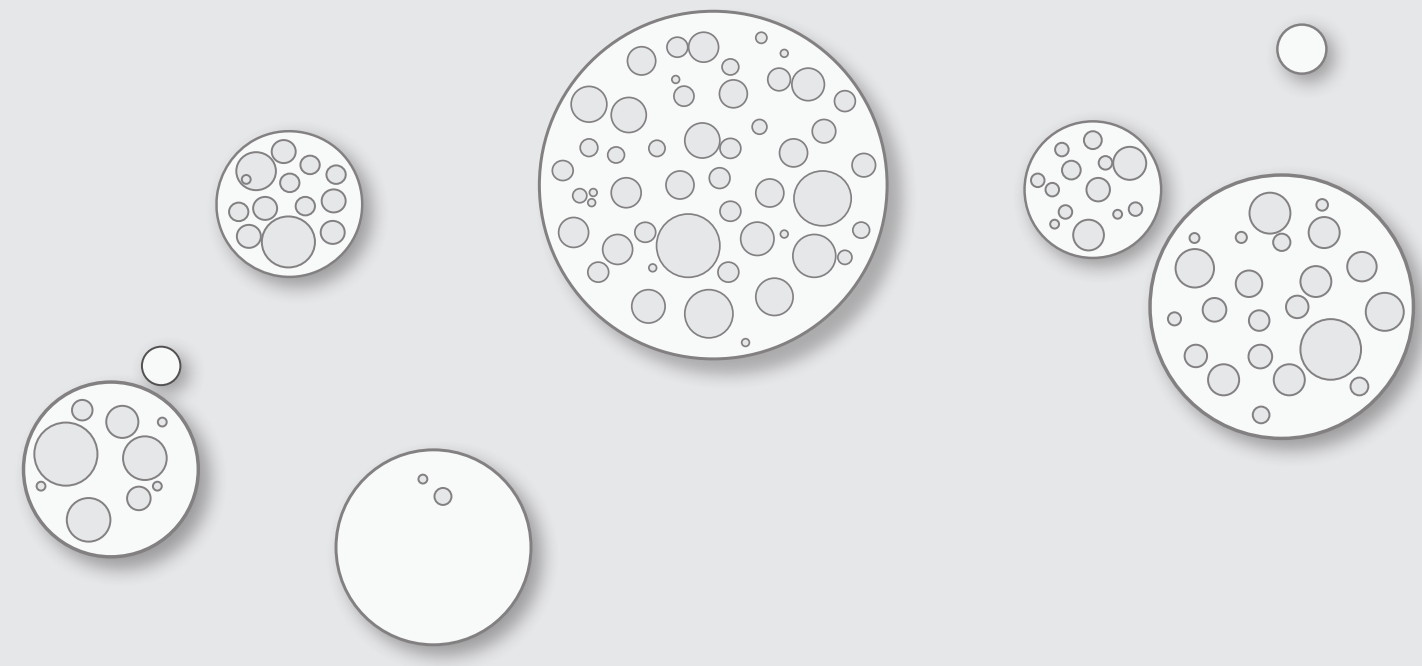

Even though it is my name on this thesis, there were so many more people involved, who accompanied and supported me over the last four years. I am feeling very blessed that I was surrounded by so many wonderful people who were part of this journey and I like to take the opportunity to express my sincere gratitude to them.

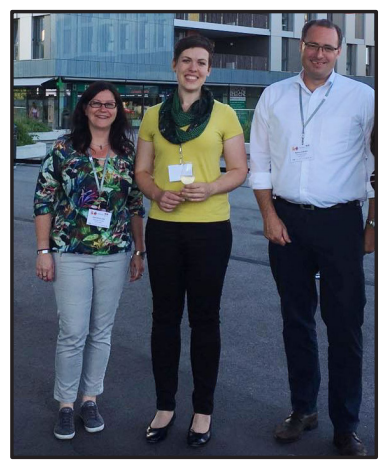

My most sincere gratitude goes to my co-promotors Markus Stieger and Elke Scholten. Thank you, Elke, for always taking time to discuss or explain things and for your thorough feedback on my manuscripts and any other text you read (or helped me to translate), and Markus, for your optimistic and reassuring attitude and your patience for guiding me through (perceived) obstacles. Elke and Markus, without your guidance, help and support, I would not have been able to complete this work as is. You have given me a lot of freedom and opportunities to give the project a direction I would feel truly comfortable with. Your encouragement and trust in me have helped me grow and develop to who I am now. You always believed in my abilities and I believe that one could not wish for better supervisors!

To my promotor Kees de Graaf, you have always been very supportive and positive throughout my PhD. Even though my topic didn't fall into your field of expertise, your encouraging words, positive feedback and continuous support made me always feel motivated to give my best.

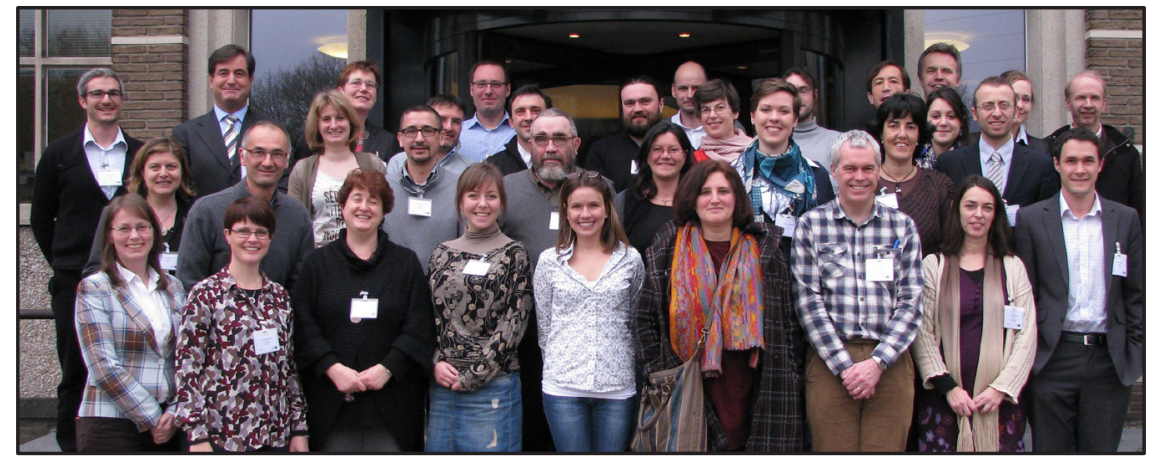

As my project was part of the European FP 7 project 'TeRiFiQ', I would also like to gratefully acknowledge the project members, coordinators and advisory board members, among which Christian Salles, Yohan Lecuona, Peter Wilde, Natalia PerezMoral, Igor Bodnar, Gilles Ferron, Harold Bult, Janny van Gijssel, Eva Veiseth-Kent, Carol Mosca, and many others. Thanks to you, I was always looking forward to our meetings! Thank you for our talks, collaborations, and exchange of knowledge and experience, for your feedback and inspiration I took home from our meetings! 
I also like to express my sincere thanks to Betina Piqueras Fiszman for your help, motivation, patience and encouraging attitude when I was struggling with the statistics part of the sensory projects. Thank you for our collaboration and for being a co-author of two of my manuscripts! I'm very grateful for having met you and having had the opportunity to work with you!

I would also like to thank Anna Schuch, Sami Sahin and Nympha de Neve for our collaborations. It was great to work with and learn from you! Herman de Beukelaer and Willem Vogelzang, thank you for running this incredible amount of DSC measurements for my students and me! Dione Bouchaut, thank you for your feedback and help when preparing my sensory studies! Miranda de Beus and Harry Baptist, I would like to thank you for your technical support and solving any problems in the lab, and Els Jansen for helping me with all kinds of administrative things! Ook hartelijk bedankt om bijna elke dag met mij Nederlands te oefenen!

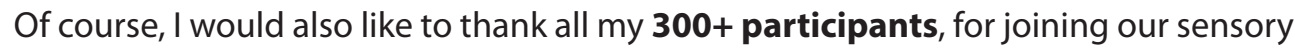
studies to taste muffins and emulsions! It is because of you that we obtained new insights in fat perception of fat-reduced products!

I would like to thank my students Ilse-Marte de Leeuw, Simone Freriks, Maaike Renssen, Marijn van Muijden, Marie-Luise Puhlmann, Josien Noppers, Arend van Rooij, Maria Montero Diaz, Jos Pols and Lisa Verkaaik whom I supervised for their theses. You are all talented, young people, with whom it has been a pleasure to work! Thank you for your motivation, curiosity, and commitment - I very much enjoyed learning from and with you, also about myself, and I am very grateful for this experience! The Sensory Science and Eating Behaviour (SSEB) group, thank you for good times we had at SSEB and your support of our rally team in 2013! Thank you also to Cornelia Bree-Evers, Gea Brussen and Jasmijn Mater for helping me out with all kind of organisational things.

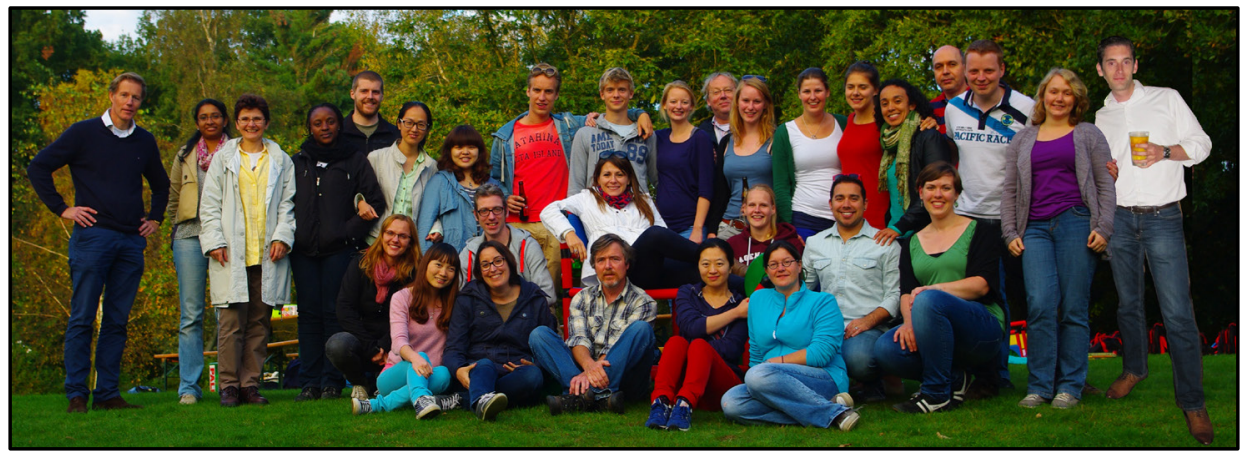


I have been very happy and fortunate to have not only been part of the Sensory Science group, but also - and actually most of the time as a PhD student - part of the Food Physics group, where I had an absolutely wonderful time. From the moment I joined you, I felt so welcome that working in this group was the best thing that could happen to me during these four years! Thank you for all the drinks and 'borrels', parties and dinners, BBQ's, 'labuitjes', PhD-weekends, PhD-trip, holidays, and all other social events
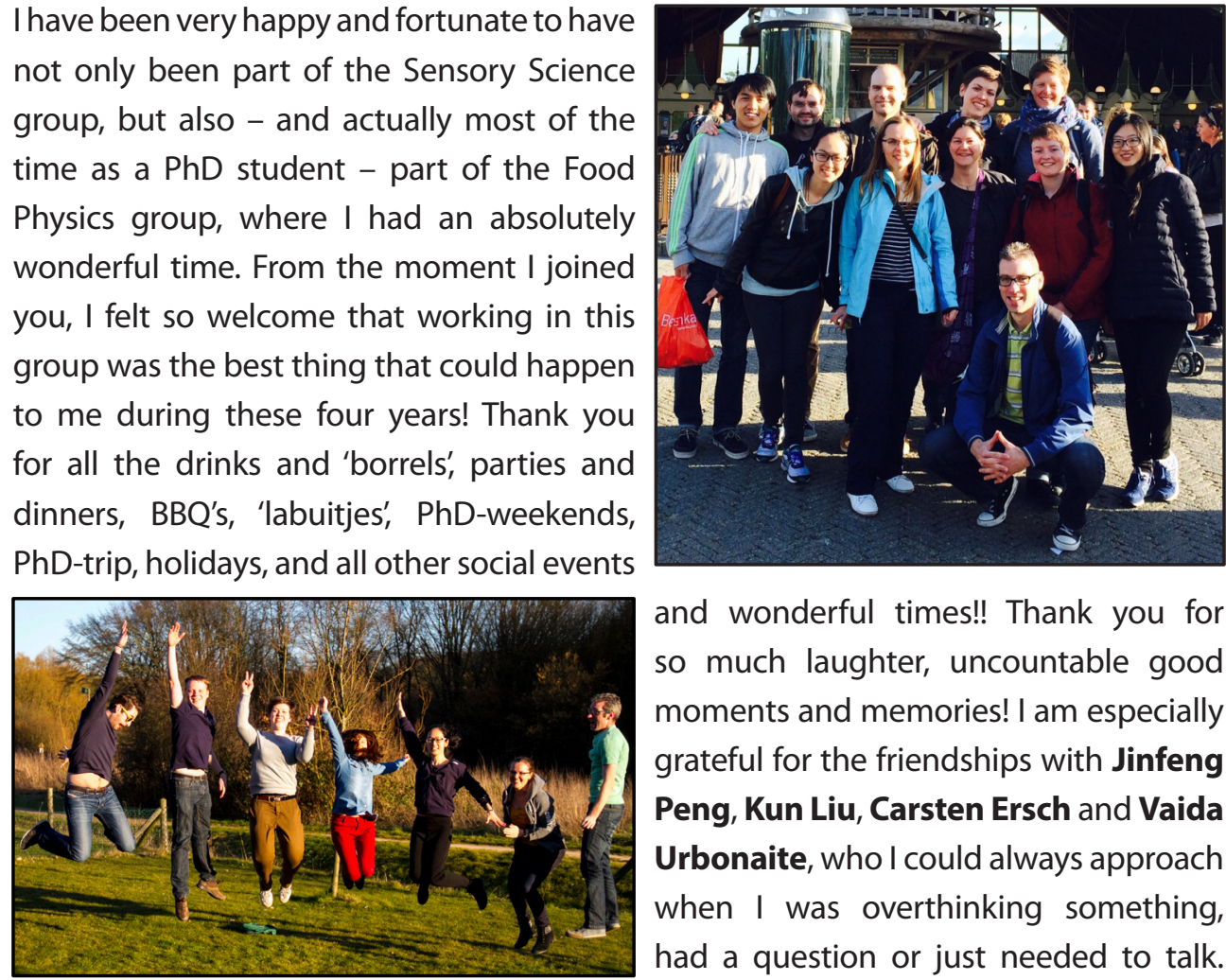

and wonderful times!! Thank you for so much laughter, uncountable good moments and memories! I am especially grateful for the friendships with Jinfeng Peng, Kun Liu, Carsten Ersch and Vaida Urbonaite, who I could always approach when I was overthinking something, had a question or just needed to talk.

Thank you for sharing your time, distracting me when I was stressing out, for making me laugh and being such wonderful friends! Thank you, Auke de Vries, for your great humour and impish poker face, for organizing the PhD-weekend in Maastricht together and for always looking for a solution (or at
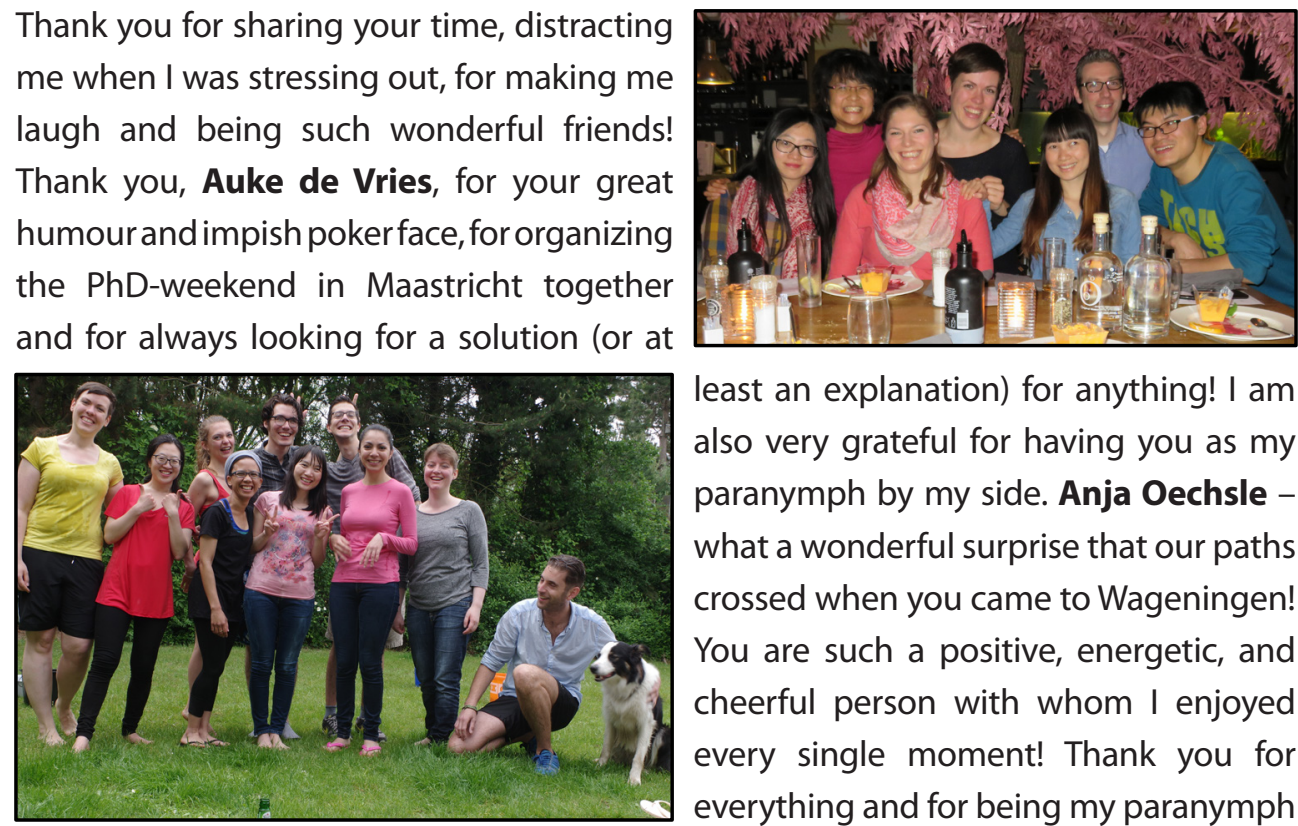

least an explanation) for anything! I am also very grateful for having you as my paranymph by my side. Anja Oechsle what a wonderful surprise that our paths crossed when you came to Wageningen! You are such a positive, energetic, and cheerful person with whom I enjoyed every single moment! Thank you for everything and for being my paranymph on this special day! 


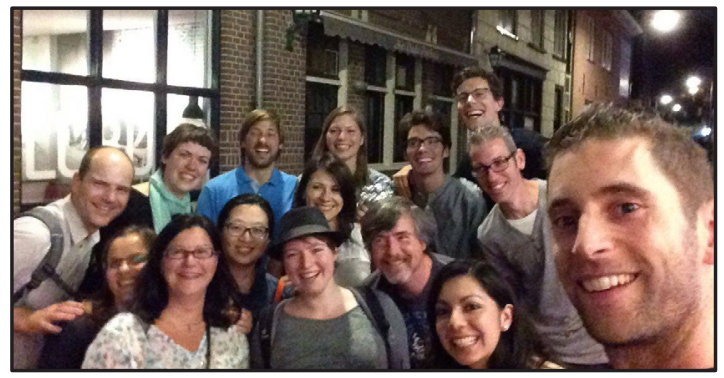

I also like to thank all my other colleagues for their help, support, fun and laughter both at work and outside work:

Els Jansen, Harry Baptist, Miranda de Beus, Erik van der Linden, Paul Venema, Leonard Sagis, Guido Sala, Tijs Rovers, Carol Mosca, Maria Tzoumaki, Marco Santagiuliana, Monica Aguayo Mendoza, Arianne van Eck, Philipp Fuhrmann, Leen Sturtewagen, Belinda Dewi, Claudine Diedericks, Pauline van Leusden, Alev Ince, Jacob Bouman, Laura Oliver Hernandez, Zhili Wan, Dilek Sağlam, Min Chen, Nam-Phuong Hua, Claire Munialo, and Lenka Tonneijck-Srpova. You all supported, helped and inspired me in many different ways for which I am very grateful and I am very glad for having been part of this wonderful group!

I also want to take the opportunity to thank my friends outside work. Lisette Bakker and Angélica Tamayo Tenorio for being great, caring housemates, who made living in Wageningen an even better experience. It was so much fun to do gardening, play Rummy Cup, go to DeBongerd or cook together.
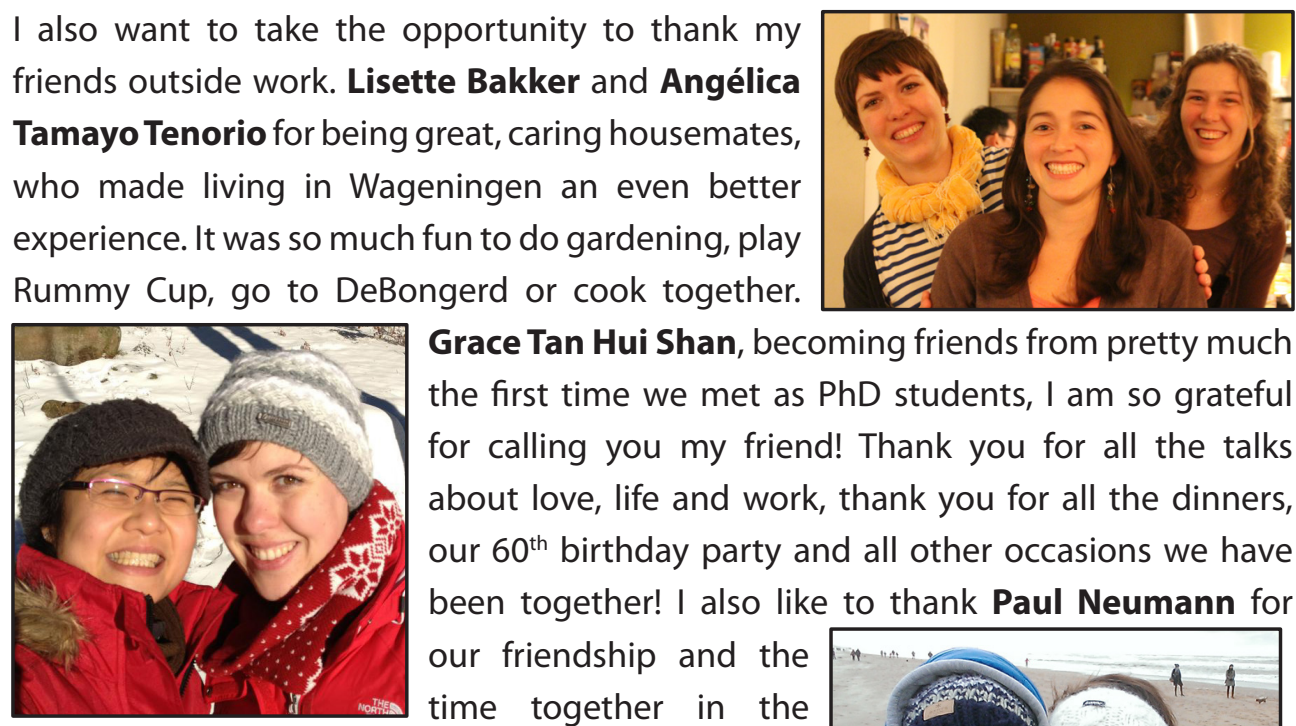

Grace Tan Hui Shan, becoming friends from pretty much the first time we met as PhD students, I am so grateful for calling you my friend! Thank you for all the talks about love, life and work, thank you for all the dinners, our $60^{\text {th }}$ birthday party and all other occasions we have been together! I also like to thank Paul Neumann for our friendship and the time together in the Netherlands! You touched my soul and through our friendship I have found more to myself - it is hard to express how thankful I am for this. Ricarda Enke, it is wonderful to share the same passion and fascination for the ocean, waves and dunes - I loved our walks

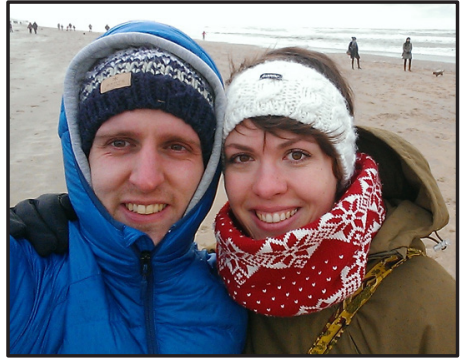
and talks along the beach in Bloemendaal-Parnassia, getting lost in the National Park or spending a day at the sauna. Thank you for always being willing to listen, honest and direct! I would also like to thank Nicole Bargmann, Benjamin Meschede and Anja 
Böhme, Carole and George Kabbani, Tanja Fritsche, Blanca Salinas y Roca and Samuel de la Fuente, and Mark and Sandra Intemann. Although we don't see each other that often, I truly enjoy every moment with you - discovering new places, trying new foods, sharing ideas and dreams, being encouraged and inspired by you - I cannot express in words how grateful I am
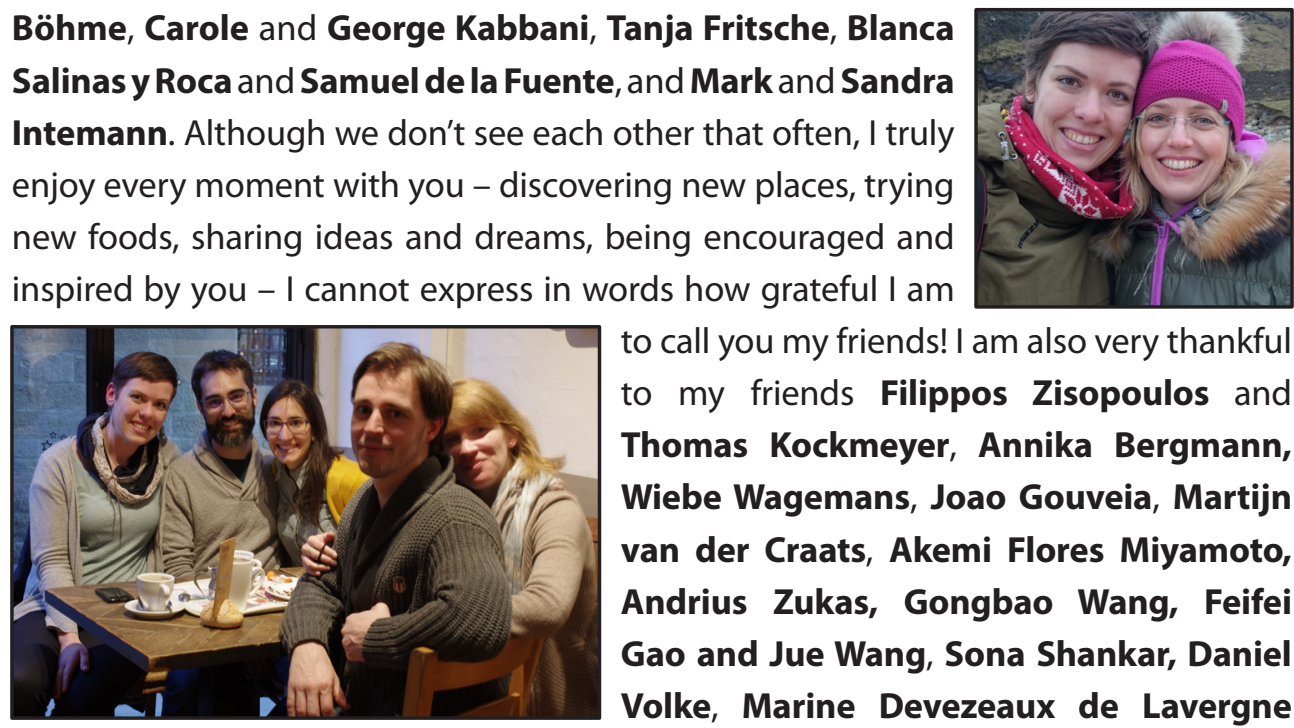

to call you my friends! I am also very thankful to my friends Filippos Zisopoulos and Thomas Kockmeyer, Annika Bergmann, Wiebe Wagemans, Joao Gouveia, Martijn van der Craats, Akemi Flores Miyamoto, Andrius Zukas, Gongbao Wang, Feifei Gao and Jue Wang, Sona Shankar, Daniel Volke, Marine Devezeaux de Lavergne and Agustin Rodriguez Fraticelli, Lotte van der Zanden, Louise den Uijl, Geraldine Avila, Eline Bakhuizen, Iliana Boshuizen-Gkini, Pey Sze (Apple) Teo, Ans and Ferry Cover, Elisa Lopez Legarreta and Jan van Bakel, Jullie Caramelle and the VLAG PhD council. Thank you for all the good times, great conversations, lunch breaks, cakes and dinners, joint events and courses, doing

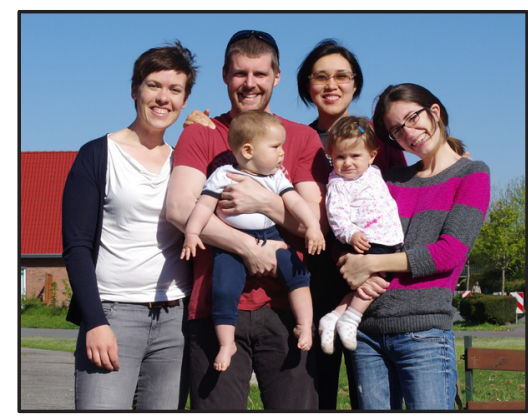
sports and countless other occasions we got together!

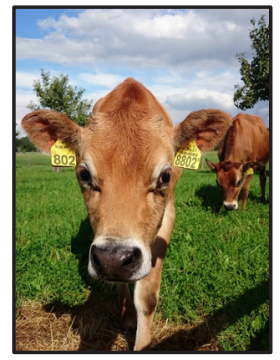

During the past four years, I also met a number of people who inspired me and through whom I learned a lot about agriculture and more. One of them is Jan Dirk van de Voort from the Remeker farm: Wat voor een inspirerende persoon jij bent!! Als stagiaire bij jullie heb ik niet alleen immens veel over koeien, kaas en duurzaamheid geleerd, maar ook dat je alles kan bereiken als je overtuigd van iets bent. Dank je wel voor alles! I also like to thank the Boerengroep association for organizing all the wonderful excursions and inspiring meetings with farmers, through which I learned so much about the different sectors and styles of farming! A special thanks also goes to Olaf Keser-Wagner

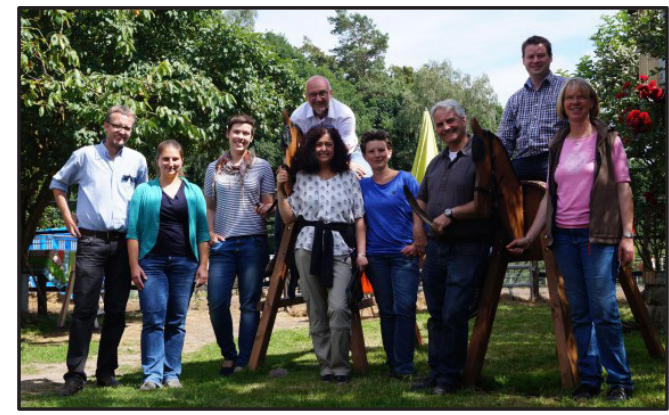


and Claudia Klebach: You introduced me to the methodology of the Erfahrungsfeld Bauernhof (EFB), in which the personal experience and reflection of consumers with the origins of their foods is key, enabling an open-minded encounter between agricultural protagonists and consumers. I am very much looking forward to be working with you and the EFB in the future!

My deepest gratitudegoes to my family. Lieber Papa, liebe Mama, liebes Schwesterherz Tatjana und lieber Opa - ihr seid das Wertvollste, was ich habe. Danke, dass ihr immer für mich da seid und an mich glaubt. Danke für eure Unterstützung aller meiner Ideen und Pläne, für eure offenen Ohren, eure Ratschläge, unsere Familienurlaube, Hilfe bei Umzügen, und für so viel mehr. Ihr macht alles in eurer Macht mögliche, um Tatjana und mich zu unterstützen. Ihr gebt uns die Freiräume, uns zu entfalten, und seid doch immer der Ort, der für uns Heimat bedeutet. Danke, dass ihr immer für uns da seid.
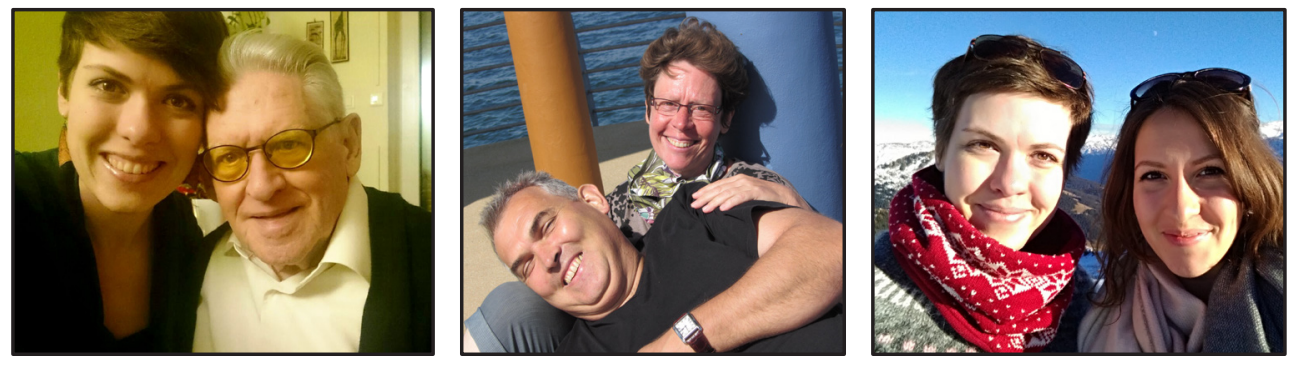

Thank you for everything!

Anika 


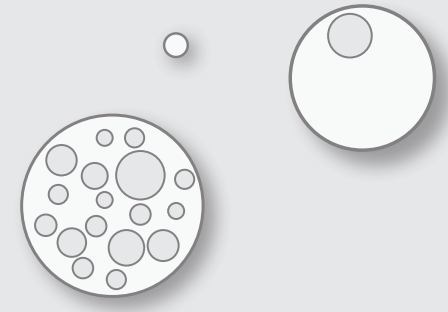

About the author
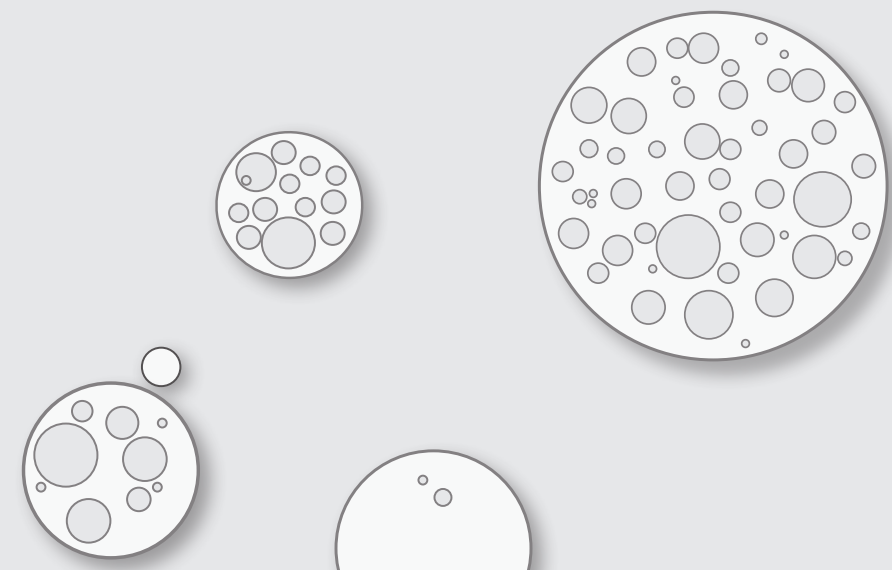

○

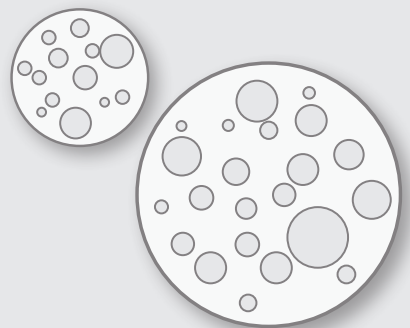





\section{Curriculum Vitae}

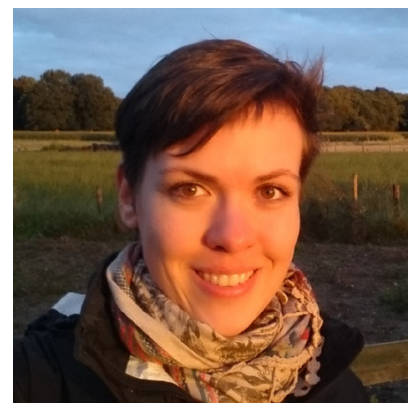

Anika Katharina Liselotte Oppermann was born on $14^{\text {th }}$ of December 1985 in Hildesheim (Germany). After finishing her secondary school in her hometown in 2005, Anika moved to Lemgo (Germany) to study food technology with emphasis bakery and confectionery technology at the University of Applied Sciences Ostwestfalen-Lippe. In her last year of her Bachelor studies, she moved to Barcelona (Spain) for an Erasmus semester at the Autonomous University of Barcelona and wrote her BSc thesis during a placement at the research and development department of Panrico. Anika graduated from her BSC in 2009. Having gotten a taste for experiencing other cultures, she enrolled for the European Masters in Food Studies, an international MSc programme organized by Wageningen University, with courses at University College Cork (Ireland), AgroParisTech (France) and Lund University (Sweden). Anika performed her MSc thesis at the Global Food Research department of Cargill in Vilvoorde (Belgium). After obtaining her MSc degree in 2012, she was appointed as a PhD candidate at the groups of Sensory Science and Eating Behaviour, and Physics and Physical Chemistry of Foods at Wageningen University. Her work was part of the European FP7-project TeRiFiQ. The results of this research are presented in this thesis.

Anika can be contacted by email: anika.oppermann@gmx.de. 


\section{List of publications}

A.K.L. Oppermann, M. Renssen, A. Schuch, M. Stieger, E. Scholten. Effect of gelation of inner dispersed phase on stability of $\left(\mathrm{w}_{1} / \mathrm{o} / \mathrm{w}_{2}\right)$ multiple emulsions. Food Hydrocolloids (2015), 48, 17-26

A.K.L. Oppermann, B. Piqueras-Fiszman, C. de Graaf, E. Scholten, M. Stieger. Descriptive sensory profiling of double emulsions with gelled and non-gelled inner water phase. Food Research International (2016), 85, 215-223

A.K.L. Oppermann, C. de Graaf, E. Scholten, M. Stieger, B. Piqueras-Fiszman. Comparison of Rate-All-That-Apply (RATA) and Descriptive sensory Analysis (DA) of model double emulsions with subtle perceptual differences. Food Quality and Preference (2017), 56, 55-68

A.K.L. Oppermann, L.C. Verkaaik, M. Stieger, E. Scholten. Influence of double $\left(\mathrm{w}_{1} / \mathrm{o} / \mathrm{w}_{2}\right)$ emulsion composition on lubrication properties. Food \& Function (2016), DOI: 10.1039/c6fo01523a

A.K.L. Oppermann, J.M.E. Noppers, M. Stieger, E. Scholten. Effect of outer water phase composition on oil droplet size and yield of double $\left(w_{1} / o / w_{2}\right)$ emulsions. Submitted. 


\section{Overview of completed training activities}

\section{Discipline-specific courses}

\section{Conferences}

2014 Food Structure \& Functionality Symposium

Food Colloids Conference

Food Oral Processing Conference

2015 Delivery of Functionality in Complex Food Systems Symposium Pangborn Sensory Science Symposium

2016 Food Colloids Conference

Food Oral Processing Conference
Amsterdam (NL)

Karlsruhe (DE)

Wageningen $(\mathrm{NL})$

Paris (FR)

Gothenburg (SE)

Wageningen (NL)

Lausanne $(\mathrm{CH})$
$\mathrm{O}, \mathrm{P}$

$\mathrm{O}, \mathrm{P}$

$\mathrm{O}, \mathrm{P}$

$\mathrm{P}$

P

Other courses and training activities

2012 Final FEl-symposium "Fat perception and satiety regulation" Regulation of Energy Intake: The role of product properties

2013 Advanced Food Analysis

Training at the Institute of Food Research (IFR)

Industrial Food Proteins

TeRiFiQ - Annual Meeting

Training at the Karlsruhe Institute of Technology (KIT)

Sensory perception and food preference

2014 TeRiFiQ - Annual Meeting

2015 TeRiFiQ - Annual Meeting

TeRiFiQ - Final Symposium

O: Oral presentation, P: Poster presentation
Bonn (DE)

Wageningen (NL)

Wageningen (NL)

Norwich (UK)

Wageningen (NL)

Ede (NL)

Karlsruhe (DE)

Wageningen (NL)

Ås (NO)

Norwich (UK)

Milan (IT)

\section{General courses}

2012 VLAG PhD week

Information literacy incl. EndNote introduction

Baarlo (NL)

Wageningen (NL)

2013 Data management planning

Wageningen (NL)

Mobilising your scientific network

Wageningen (NL)

Voice matters - Voice and presentation skills training

Wageningen (NL)

Techniques for writing and presenting a scientific paper

Wageningen (NL)

2014 Philosophy and ethics of food science \& technology

Wageningen (NL)

Multivariate analysis

Wageningen (NL)

2015 Entrepreneurship in- and outside science

Wageningen (NL)

Adobe InDesign

Wageningen (NL) 


\section{Optional courses and activities}

2013

Preparation of a research proposal

2014

PhD study tour (US, Canada)

2014 - 2016 Committee member of the VLAG PhD council 



\section{Colophon}

The work presented in this thesis was funded by the European Union Seventh Framework Programme (FP 7/2007-2013) under grant agreement number 289397 (TeRiFiQ project).

Financial support from Wageningen UR for printing this thesis is gratefully acknowledged.

Cover: Kun Liu \& Anika Oppermann

Layout: Anika Oppermann

Printing: GVO drukkers \& vormgevers, Ede (gvo.nl)

Copyright: Anika Oppermann, 2017 